\title{
Measuring Crustal Seismic Anisotropy through Shear Wave Splitting
}

By Kenny Mensah Graham

\author{
A thesis \\ submitted to Victoria University of Wellington \\ in fulfilment of the requirements for the degree of \\ Doctor of Philosophy in Geophysics.
}

Victoria University Wellington

2020 

"Simplicity is the ultimate sophistication."

Leonardo da Vinci

"Obra pa gya owura kwan."

A Ghanaian adage that says "good deeds and efforts, pay at the end of the journey" 



\section{Abstract}

This thesis involves the study of crustal seismic anisotropy through shear wave splitting. For the past three decades, shear wave splitting (SWS) measurements from crustal earthquakes have been utilised as a technique to characterize seismic anisotropic structures and to infer in situ crustal properties such as the state of the stress and fracture geometry and density. However, the potential of this technique is yet to be realized in part because measurements on local earthquakes are often controlled and/or affected by physical mechanisms and processes which lead to variations in measurements and make interpretation difficult. Many studies have suggested a variety of physical mechanisms that control and/or affect SWS measurements, but few studies have quantitatively tested these suggestions. This thesis seeks to fill this gap by investigating what controls crustal shear wave splitting (SWS) measurements using empirical and numerical simulation approaches, with the ultimate aim of improving SWS interpretation. For our empirical approach, we used two case studies to investigate what physical processes control seismic anisotropy in the crust at different scales and tectonic settings. In the numerical simulation test we simulate the propagation of seismic waves in a variety of scenarios.

We begin by measuring crustal anisotropy via SWS analysis around central New Zealand, where clusters of closely-spaced earthquakes have occurred. We used over 40,000 crustal earthquakes across 36 stations spanning close to $5 \frac{1}{2}$ years between 2013 and 2018 to generate the largest catalog of high-quality SWS measurements $(\sim 102,000)$ around the Marlborough and Wellington region. The size of our SWS catalog allowed us to perform a detailed systematic analysis to investigate the processes that control crustal anisotropy and we also investigated the spatial and temporal variation of the anisotropic structure around the region. We observed a significant spatial variation of SWS measurements in Central New Zealand. We found that the crustal anisotropy around Central New Zealand is confined to the upper few kilometers of the crust, and is controlled by either one mechanism or a combination of more than one (such as structural, tectonic stresses and gravitational stresses). The high correspondence between the orientation of the maximum horizontal compressive stress calculated from gravitational potential energy from topography and average fast polarization orientation around the Kaikōura region suggests that gravitationally induced stresses control the crustal anisotropy in the Kaikōura region. We suggest that examining the effect of gravitational stresses on crustal seismic anisotropy should not be neglected in future 
studies. We also observed no significant temporal changes of the state of anisotropy over the $5 \frac{1}{2}$ year period despite the occurrence of significant seismicity.

For the second empirical study, we characterized the anisotropic structure of a fault approaching failure (the Alpine Fault of New Zealand). We performed detailed SWS analysis on local earthquakes that were recorded on a dense array of 159 three component seismometers with inter-station spacing about $30 \mathrm{~m}$ around the Whataroa valley, New Zealand. The SWS analysis of data from this dense deployment enabled us to map the spatial characteristics of the anisotropic structure and also to investigate the mechanisms that control anisotropy in the Whataroa valley in the vicinity of the Alpine Fault. We observed that the orientation of the fast direction is parallel to the strike of the Alpine Fault trace and the orientations of the regional and borehole foliation planes. We also observed that there was no significant spatial variation of the anisotropic structure as we move across the Alpine Fault trace from the hanging wall to the footwall. We inferred that the geological structures, such as the Alpine Fault fabric and foliations within the valley, are the main mechanisms that control the anisotropic structure in the Whataroa valley.

For our numerical simulation approach, we simulate waveforms propagating through an anisotropic media (using both 1-D and 3-D techniques). We simulate a variety of scenarios, to investigate how some of the suggested physical mechanisms affect SWS measurements. We considered (1) the effect on seismic waves caused by scatterers along the waves' propagation path, (2) the effect of the earthquake source mechanism, (3) the effect of incidence angle of the incoming shear wave. We observed that some of these mechanisms (such as the incidence angle of the incoming shear wave and scatterers) significantly affect SWS measurement while others such as earthquake source mechanisms have less effect on SWS measurements. We also observed that the effect of most of these physical mechanisms depends on the wavelength of the propagating shear wave relative to the size of the features. There is a significant effect on SWS measurements if the size of physical mechanism (such as scatterers) are comparable to the wavelength of the incoming shear wave. With a larger wavelength, the wave treats the feature as a homogeneous medium. 


\section{Acknowledgments}

I wish to acknowledge, first and foremost, the patience, tutelage and support of my supervisors Martha Savage and Richard Arnold. I have gained greatly from their immense knowledge and sheer tenacity towards this research. I am really indebted to their continuous support and patience throughout this journey. Me da ase paaa (Thank you very much).

I am grateful for the financial support I have received from VUW-Doctoral Scholarship and the Earthquake Commission of New Zealand (EQC Grant number: 18/755). I am also grateful to the New Zealand eScience Infrastructure (project number "nesi00312") for providing me with computational time to run my simulations. I am grateful to Dr. Simon Lamb, Dr. Carolyn Boulton, Dr. Katrina Jacobs, Dr. Calum Chamberlain and Hamish Hirschberg for their discussions and helpful feedback. I thank Hubert J. Zal, Tomomi Okada, Yoshihisa Iio and Satoshi Matsumoto for your thoughtful discussions, suggestions and invaluable input to the submitted manuscript. Waveform data used for this study were provided by New Zealand GeoNet, Disaster Prevention Research Institute, Kyoto University, Japan (Grant Number: THK01), and the 2016 Whataroa Active Source Seismic Experiment. I would like to acknowledge everyone involved in acquiring these dataset.

To my office-mates and fellow graduate students, Konstantinos, Hubert, Bryant, Steven, Dominic, Konrad, Laura-May, Weiwei, El, Jesse and Katie (and others that I may have missed) you have been awesome throughout these years. Thank you, Aleksandr Beliaev and Adrian Benson for your expertise and technical support. I am also grateful to the staff and students of the school of Mathematics and Statistics, VUW for their support.

I express my deepest appreciation to my family. My success would not have been possible without their deep love, unwavering support and constant prayers. Nana Ama Anowiah, Madam Ernestina Eshun, Mr John Mantey, Madam Evelyn Doe Budu, Robert, Josephine, Victoria and Emmanuel thank you for the support. To my friend and brother Dr. Setor Donne Novieto thank you so much for the encouragement. To Mr. Osei Amoafo and family, Dr. Tom Foster and family, and Nukunya Atsu Dogbe thank you for the support throughout this journey. The Ghanaian community in Wellington and my friends have also been a great support in this journey and I acknowledge them. 
This journey would not have been complete without the smiles and encouragements from my lovely wife Mrs. Abigail Graham. Thank you for the support through out this journey. To Kekeli Graham, my light, you continue to brighten my dark moments. To our unborn son (Paa Willie) you gave me the hope to push harder. I can't wait to have you in my arms. 


\section{Contents}

$\begin{array}{ll}\text { Abstract } & \text { i }\end{array}$

Acknowledgments $\quad$ iii

List of Figures $\quad$ xi

List of Tables $\quad$ xiii

1 Introduction and background 1

1.1 Introduction . . . . . . . . . . . . . . . . . . . . . . . 1

1.1.1 Motivation . . . . . . . . . . . . . . . . 1

1.1.2 Thesis structure . . . . . . . . . . . . . . . 3

1.2 Background . . . . . . . . . . . . . . . . . . . 5 5

1.2.1 Tectonic setting . . . . . . . . . . . . . . 5

1.3 Seismic anisotropy and shear wave splitting _ . . . . . . . . . . . 9

1.3.1 Seismic anisotropy . . . . . . . . . . . . . . . . 9 9

1.3.2 Shear wave splitting . . . . . . . . . . . . . 16

1.3.3 Measuring crustal anisotropy . . . . . . . . . . . . . . 18

1.3.4 Variations in measuring seismic anisotropy using shear wave splitting ........................... 20

1.3.5 Variation in SWS measurements . . . . . . . . . . . . . . 22

2 Data and methods $\quad 27$

2.1 Data . . . . . . . . . . . . . . . . . . . . 27

2.1.1 Seismic network . . . . . . . . . . . . . . . . . 27

2.2 Methods . . . . . . . . . . . . . . . . . . . . 28

2.2.1 Estimating shear wave splitting parameters . . . . . . . . . . . 28

2.2.2 Spatial averaging of fast direction measurements . . . . . . . . . 38

2.2.3 SpecFem3D: Spectral finite element method . . . . . . . . . . . 41

3 Seismic anisotropy around central New Zealand 49

3.1 Introduction . . . . . . . . . . . . . . . . . . . . . 50

3.2 Tectonic setting and seismicity . . . . . . . . . . . . . 55

3.3 Data and methodology . . . . . . . . . . . . . . . . 58

3.3 .1 Data . . . . . . . . . . . . . . . 58 


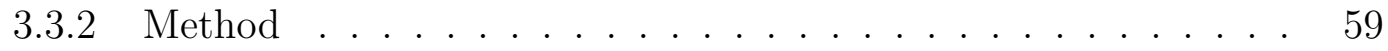

3.4 Results . . . . . . . . . . . . . . . . . 67

3.4.1 Spatial averaging . . . . . . . . . . . . . . . 71

3.5 Discussion . . . . . . . . . . . . . . . . . . . . 74

3.5.1 Overall station trends . . . . . . . . . . . . . . . 74

3.5.2 Search for temporal variation . . . . . . . . . . . . . . 77

3.5.3 Depth extent of anisotropy . . . . . . . . . . . . . . . . . 82

3.5.4 Discriminating between stress and structural anisotropy . . . . . 83

3.5.5 Comparing fast directions with stress indicators . . . . . . . . 86

3.5.6 Comparing plane of maximum shear stress with fast directions . 88

3.5.7 What controls seismic anisotropy in central New Zealand . . . . 89

3.6 Conclusions . . . . . . . . . . . . . . . . . . . . . . . . . . 92

4 Anisotropic Structure in the Whataroa Valley 93

4.1 Introduction . . . . . . . . . . . . . . . . . . . . . . . . . . . 94

4.1 .1 Previous studies . . . . . . . . . . . . . . . . . 996

4.1.2 Tectonics and seismicity around the Whataroa Valley . . . . . . 99

4.2 Data and methodology . . . . . . . . . . . . . . . . . . . . . . 102

4.2 .1 Data . . . . . . . . . . . . . . . . . 102

4.2 .2 Methods . . . . . . . . . . . . . . . . . . . 103

4.3 Results . . . . . . . . . . . . . . . . . . . . . . 110

4.3.1 Event locations . . . . . . . . . . . . . . . . . . . 110

4.3.2 General overview of SWS measurements . . . . . . . . . . . 110

4.4 Discussion . . . . . . . . . . . . . . . . . . . 116

4.4.1 Spatial variability in seismic anisotropy within the valley . . . . 118

4.4.2 Mechanisms of anisotropy in the Whataroa Valley . . . . . . . . 122

4.5 Conclusions . . . . . . . . . . . . . . . . . . . . . . . . 124

5 What Causes Variations in SWS Measurements 125

5.1 Introduction . . . . . . . . . . . . . . . . . . 126

5.2 Method . . . . . . . . . . . . . . . . . . . 129

5.2 .1 1-D modeling . . . . . . . . . . . . . . . . . . . . . 129

5.2 .2 Mesh design for SpecFEM3D . . . . . . . . . . . . . . 131

5.2.3 Implementation and verification of anisotropic models . . . . . . 132

5.2 .4 Model selection . . . . . . . . . . . . . . . . . . . 137

5.2 .5 Source parametrization . . . . . . . . . . . . . . . . 138

5.2.6 Models for testing the effect of incidence angle . . . . . . . . . . 142

5.3 Results and Discussion . . . . . . . . . . . . . . . . . . . . 145

5.3.1 Effect of incidence angle of the incoming shear wave on SWS . . 145

5.3 .2 Effect of varying source mechanism . . . . . . . . . . . . . . . . 149 
5.3.3 Effect of scatterers on SWS measurements . . . . . . . . . . . . 152

5.3.4 Effect of noise on SWS measurements . . . . . . . . . . . . . 164

5.4 Conclusions . . . . . . . . . . . . . . . . . . . 167

6 Summary $\quad 169$

6.1 Future research . . . . . . . . . . . . . . . . . . . . 174

$\begin{array}{ll}\text { Appendices } & 177\end{array}$

$\begin{array}{ll}\text { A Supporting Information for Chapter } 3 & 179\end{array}$

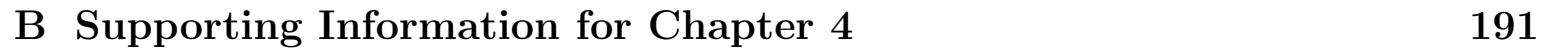

C Supporting Information for Chapter 5 205 


\section{List of Figures}

1.1 Basement rocks of New Zealand . . . . . . . . . . . . . . . 6

1.2 Regional tectonics . . . . . . . . . . . . . . . . . . . . . . 8

1.3 Anisotropy at all scales . . . . . . . . . . . . . . . . . . . . 10

1.4 Shear Wave Splitting . . . . . . . . . . . . . . . . . . . . 17

1.5 Illustrating of stress induced and structural controlled anisotropy . . . 19

1.6 Illustration of the principle of cycle skipping . . . . . . . . . . . . 23

2.1 Split and unsplit waveform . . . . . . . . . . . . . . . . . 30

2.2 Horizontal particle motion of the waveform for split (A) and unsplit (B) waveform . . . . . . . . . . . . . . . . 31

2.3 Example of MFAST diagnostic plots for a good quality measurement . 32

2.4 Example of MFAST diagnostic plots; good measurement for real data . 33

2.5 Contour plot of $\phi$ versus $\delta t \ldots \ldots \ldots$. . . . . . . . . . 36

2.6 MFAST diagnostic plots null measurements . . . . . . . . . . . . 38

2.7 Example of MFAST diagnostic plots; null measurement of real data . . 39

2.8 Schematic workflow of SPECFEM3D simulation . . . . . . . . . . . . 43

2.9 Subdividing the model . . . . . . . . . . . . . . . . . 44

2.10 The geometry of a hexahedral finite element (deformed cube) . . . . . . 45

2.11 GLL point and Lagrange interpolants . . . . . . . . . . . . . . . . . . . 46

3.1 Kaikōura tectonic . . . . . . . . . . . . . . . . . . . . . 56

3.2 Distribution of earthquake epicenters . . . . . . . . . . . . . . . 60

3.3 Distributions of S-picks errors relative to analyst pick . . . . . . . . . . 62

3.4 Rose diagrams of Fast direction measurements . . . . . . . . . . . . . 70

3.5 Rose diagram for before and after Kaikōura measurements . . . . . . . 72

3.6 Spatial averages of fast direction measurements . . . . . . . . . . 73

3.7 Quantitative comparison for before and after Kaikōura earthquake . . . 75

3.8 Time series plot delay times, $\delta t$ and $\mathrm{V}_{\mathrm{p}} / \mathrm{V}_{\mathrm{s}}$ ratio . . . . . . . . . 79

3.9 State of anisotropy over the $5 \frac{1}{2}$ year period . . . . . . . . . . . 81

3.10 Scatter plot of delay times versus depth for station KHZ . . . . . . . . 83

3.11 Quantitative comparison between $\phi$ and fault orientations . . . . . . 85

3.12 Quantitative comparison between $\phi$ and gravitational stress orientation 87

3.13 Quantitative comparison between $\phi$ and maximum shear plane . . . . . 90

3.14 Summary of the mechanisms controlling anisotropy . . . . . . . . . . . 91 
4.1 Overview map of Whataroa . . . . . . . . . . . . . 100

4.2 Seismograph deployment across the Whataroa Valley . . . . . . . . . . 101

4.3 Example of detected waveform . . . . . . . . . . . . . . . . . 106

4.4 Earthquakes used for analysis . . . . . . . . . . . . . . . . 107

4.5 Distributions of delay times . . . . . . . . . . . . . . . . 109

4.6 The spatial distribution of $\phi$ orientation . . . . . . . . . . . . . . . . 112

4.7 The spatial distribution of averaged $\phi$ orientation . . . . . . . . . . . . 114

4.8 Spatial distribution of $\delta t$ and percentage of anisotropy . . . . . . . . . 115

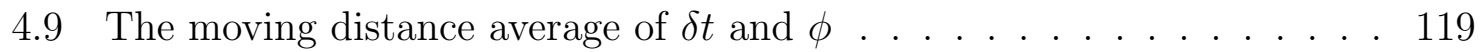

4.10 The averaged of $\phi$ orientation across the Alpine Fault . . . . . . . . . . 121

4.11 Lower hemisphere plot of fast direction and other structural data sets . 123

5.1 Geometry of receiver and source distribution used for synthetic studies 133

5.2 Comparing isotropic and anisotropic waveform. . . . . . . . . . 135

5.3 Polar view representation of different elasticity tensors . . . . . . . 136

5.4 Example of initial testing model . . . . . . . . . . . . . . . . . . . . 139

5.5 Comparing waveforms for the single and double layer model . . . . . . 140

5.6 Models used for 1-D synthetic analysis . . . . . . . . . . . . . . . . . . 143

5.8 Comparison of apparent SWS parameters with back-azimuth, 1-D case 146

5.9 Station configuration on MSAT output . . . . . . . . . . . . . . . . 147

5.10 Comparison of apparent SWS parameters with back-azimuth, 3D case . 148

5.11 Varying source mechanism . . . . . . . . . . . . . . . . . 150

5.12 Source $2013 p 543824$ showing null results . . . . . . . . . . . . . . . . . 151

5.13 Models for effect of scatterers . . . . . . . . . . . . . . . 153

5.14 Schematic diagram of to test for effect of scatterers . . . . . . . . . . 154

5.15 Waveform from station far away from a high velocity anomaly . . . . . 155

5.16 Waveform from station directly above high velocity anomaly . . . . . . 156

5.17 Waveform from station at the edge of a high velocity anomaly . . . . . 157

5.18 SWS measurements for stations directly above the scatterer . . . . . . 160

5.19 SWS measurements for stations far away from the scatterer . . . . . . . 161

5.20 SWS measurements for stations at the edge of the scatterer . . . . . . . 162

5.21 Example of MFAST diagnostic plots for scatterer . . . . . . . . . . . 163

5.22 Example of noise added waveform . . . . . . . . . . . . . 165

5.23 Effect of noise on SWS parameters . . . . . . . . . . . . . . 166

A.1 Before and after plot of Kaikōura event . . . . . . . . . . . . . . . . . . 181

A.2 Plot of data continuity . . . . . . . . . . . . . . . . . . . 182

A.3 Example of generalized phase detection GPD pick . . . . . . . . . . . 183

A.4 Distributions of S-picks error between GeoNet and GDP S-picks . . . . 184

A.5 Quadtree gridding of the study area . . . . . . . . . . . . . . 185 
A.6 Spatial averaging results of $\phi$ for before and after measurements . . . . 186

A.7 Time series of delay times, $\delta t$ and $\mathrm{V}_{\mathrm{p}} / \mathrm{V}_{\mathrm{s}}$ ratio $\ldots \ldots \ldots$. . . . . 187

A.8 Scatter plot of delay times versus path length for station KHZ . . . . . 188

A.9 Quantitative comparison between spatially averaged $\phi$ and $S_{H \max }$. . . 189

B.1 The spatial distribution of $\phi$ for event 2016q17101910 . . . . . . . . . . 197

B.2 The spatial distribution of $\phi$ for event 2016q19134730 . . . . . . . . . . 198

B.3 The spatial distribution of $\phi$ for event 2016q19172800 . . . . . . . . . 199

B.4 The spatial distribution of $\phi$ for event $2016 q 20043517$. . . . . . . . . . 200

B.5 The spatial distribution of $\phi$ for event 2016q20044700 . . . . . . . . . . 201

B.6 The spatial distribution of $\phi$ for event 2016q20181040 . . . . . . . . . . 202

B.7 The spatial distribution of $\phi$ for event 2016q21182055 . . . . . . . . . . 203

B.8 The moving distance average of $\delta t$ and $\phi$ along latitude . . . . . . . . . 204

C.4 Waveform from station away from low velocity anomaly . . . . . . . . . 209

C.5 Waveform from station on top low velocity anomaly . . . . . . . . . . . 210

C.6 Waveform from station at the edge of low velocity anomaly . . . . . . . 211 


\section{List of Tables}

1.1 Voigt notation . . . . . . . . . . . . . . . . . . . 13

1.2 Classification of anisotropic media . . . . . . . . . . . . . . . 13

3.1 Summary statistics of SWS measurements for GeoNet stations . . . . . 68

3.2 Summary statistics of SWS measurements for DPRI stations . . . . . . 69

4.1 Seismic stations used for Whataroa event detection . . . . . . . . . . . 104

5.1 Elastic tensors used in validating the model . . . . . . . . . . . . . 137

5.2 Source used for analysis . . . . . . . . . . . . . . . 141

A.1 Summary of adjusted parameters used for picking S-arrivals . . . . . . 180

B.1 STA/LTA site-specific parameters . . . . . . . . . . . . . . . . . 192

B.2 Filters Applied to Waveforms Prior to MFAST analysis . . . . . . . . . 192

B.3 Summary statistics of SWS measurements . . . . . . . . . . . . . 193

B.4 Continuation of Table B.3 . . . . . . . . . . . . . . . . 194

B.5 Continuation of Table B.4 . . . . . . . . . . . . . . . 195

B.6 Continuation of Table B.5 . . . . . . . . . . . . . . . . . . 196 


\section{Introduction and background}

The general overview and background of the thesis is presented in this chapter. $\mathrm{I}^{1}$ start with the motivation of this study followed by the research questions and thesis structure. This chapter also contains an introduction to seismic anisotropy and shear wave splitting (SWS). The chapter ends with a literature review on measuring seismic anisotropy through shear wave splitting.

\subsection{Introduction}

\subsubsection{Motivation}

In the crust, natural cracks (or fractures) play an important role in the fluid-flow and in-situ permeability, rock strength (Townend and Zoback, 2000), and geomechanical behaviour (Zoback and Zoback, 2002) of the subsurface. Thus the characterisation and knowledge of crack orientation and density is a consistent goal of geophysical studies. Seismic anisotropy has been used by Earth scientists as a tool for characterising in-situ properties of the Earth's crust (Crampin and Booth, 1989; Savage, 1999). Earthquakes in the crust are caused by the sudden rupture of rocks along faults exposed to the stress field in the Earth's crust, and occur when the accumulated stress exceeds the strength of faults or fractures in rock. Thus, crustal stresses are the immediate driving forces of earthquakes (Zoback and Zoback, 2002). Seismic anisotropy measurements using SWS have been used to estimate the horizontal principal stress orientation (Cochran and Kroll, 2015; Crampin et al., 2015; Savage et al., 2016). For decades, measuring seismic anisotropy through shear wave splitting of crustal earthquakes has been utilised as a

\footnotetext{
${ }^{1}$ Although most of the work presented here has been conducted by me, for the future chapters I am writing this thesis in the first-person plural, in recognition of the significant contributions from my supervisors.
} 
technique to infer the state of stress, fracture orientation and crack density in the crust (Crampin, 1984; Savage, 1999; Savage et al., 2016; Cochran and Kroll, 2015). However, the full potential of this technique is yet to be realized in part because measurements on local earthquakes have often shown significant variations, making interpretation difficult. Moreover, seismic anisotropy in the crust is known to be controlled by several physical processes (Boness and Zoback, 2006).

The overall goal of this thesis is to address the question: "What physical processes and/or mechanisms control or affect measurement of crustal seismic anisotropy through shear save splitting?". To answer this question, we used both empirical and numerical simulation approaches. For the empirical studies, we used experiments from two tectonic settings; (1) a regional scale $(400 \mathrm{~km} \times 300 \mathrm{~km})$ and $(2)$ very local $(4 \mathrm{~km} \times 3 \mathrm{~km})$ scale. For the numerical simulation studies, we used a series of scenarios to investigate how some physical mechanisms affect SWS measurements.

For the regional scale, we performed SWS analysis using over 40,000 crustal earthquakes recorded on 36 seismic stations to investigate the physical processes that control crustal anisotropy. We performed a detailed spatial and temporal analysis of shear wave splitting measurements with the aim of investigating what controls the anisotropic structure in central New Zealand; a complex tectonic setting where there is subduction merging to strike-slip deformation amid the complex Marlborough fault system (Van Dissen and Yeats, 1991; Anderson et al., 1993). We tried to answer the following research questions:

1. What is the lateral and vertical extent of the anisotropic structures in central New Zealand?

2. What physical processes control seismic anisotropy in a complex tectonic region? Does gravitational stress, as a result of strong topography contrast, control seismic anisotropy?

3. Did the 2013 Cook Strait earthquakes and the $2016 \mathrm{M}_{m w} 7.8$ Kaikōura earthquake sequences affect the anisotropic structure or the state of anisotropy in central New Zealand? 
In addition to answering these questions, we took advantage of the increased seismicity around the region to document in detail the anisotropic structure in central New Zealand.

For the local scale we used SWS measurements from local earthquakes near Whataroa Valley, South Island, New Zealand. The earthquakes were recorded on 159 three component $(3 \mathrm{C})$ seismometers with inter-station spacing $\sim 30 \mathrm{~m}$. We used these measurements to characterise the anisotropic structure of a fault that is considered to be approaching failure (the Alpine Fault of New Zealand Sutherland et al., 2017; Beavan et al., 2010). In addition to investigating the processes that control the anisotropic structure within the Whataroa valley, we also tried to document the anisotropic structure within the Whataroa Valley. To do this, we attempt to answer the following research questions:

1. What controls the anisotropic structure in the Whataroa Valley in the vicinity of the Alpine Fault?

2. What variation in anisotropic structure occurs as you move across the fault from the hanging wall to the foot-wall?

3. What is the spatial variability of seismic anisotropy in the Whataroa Valley?

For the numerical simulation study, we investigated the possible physical mechanisms that may cause variations in SWS measurements, with the ultimate goal of improving the shear wave splitting interpretation. To achieve this, we analyzed a series of synthetic scenarios to answer the following research questions:

1. How does varying angle of incidence affect SWS measurements?

2. How does varying fault source orientations affect SWS measurements?

3. How do scatterers affect SWS measurements?

\subsubsection{Thesis structure}

Chapter 1 (this Chapter) has two main sections; an introduction and a background. The introduction section lays out the motivation, the structure of the thesis, and a 
brief overview of the seismotectonic setting of New Zealand. The background section addresses the theoretical background of seismic anisotropy and shear wave splitting. An introduction to the possible mechanisms affecting shear wave splitting measurements is also presented here. Chapter 2 describes the data and methods used in this study. Chapters 3, 4 and 5 are each standalone projects, written and formatted with the intent of publication. Additional methodologies used in these standalone chapters are also addressed in the appropriate chapters.

Chapter 3 is published in Geophysical Journal International entitled, "Spatiotemporal Analysis of Seismic Anisotropy Associated with the Cook Strait and Kaikōura Earthquake Sequences in New Zealand". This chapter seeks to investigate the physical processes controlling crustal anisotropy around the Marlborough and Wellington region and to explore any possible temporal variations in shear wave splitting associated with the 2013 Cook Strait and the 2016 Kaikōura earthquake.

In Chapter 4 we characterized the anisotropic structure and infer the likely mechanisms that control seismic anisotropy in the Whataroa Valley in the vicinity of the Alpine Fault (a fault approaching failure).

In Chapter 5 we investigated how the suggested physical mechanisms affect SWS measurements using simulated waveforms propagating through an anisotropic media. We simulate a variety of scenarios to investigate how incidence angle of the incoming shear wave, earthquake source mechanism, and scatterers affect SWS measurements.

Lastly, Chapter 6 summarizes the findings of the three main chapters and suggests potential research directions that emerge from these studies. 


\section{$1.2 \quad$ Background}

This section describes the tectonic setting around central New Zealand and the Whataroa valley. We also introduce seismic anisotropy and shear wave splitting in this section.

\subsubsection{Tectonic setting}

Chapters 3 and 4 provide detailed overviews of their respective tectonic settings. This section provides a brief overview of the tectonic setting in New Zealand. New Zealand is situated at the boundary between the Australian and Pacific tectonic plates. New Zealand's tectonic setting is characterized by two subduction systems of opposite polarity, connected by an area of oblique continental convergence (Figure 1.1 and inset of Figure 1.2). Moving from north to south, the setting starts from the westward subduction (and intra-arc rifting) in the North Island at the Hikurangi subduction zone, to a component of strike-slip motion on the Marlborough Fault Zone, transpressional collision along the Alpine Fault and finally back to (eastward) subduction again at the Puysegur Trench (Figure 1.1 and inset of Figure 1.2) offshore Fiordland (Beavan et al., 2016, and references therein). In the North Island, the Pacific plate subducts obliquely westward beneath the Australian plate at the Hikurangi subduction margin with a relative plate motion of $\sim 39$ to $48 \mathrm{~mm} / \mathrm{yr}$, increasing northward (Beavan et al., 2016; Clark et al., 2017; Litchfield et al., 2017). The Hikurangi subduction margin strikes in the NE-SW direction, and the plates converge obliquely (almost E-W) to the strike (Williams et al., 2013). Geodetic data around the Marlborough fault system indicate that the relative motion between the Pacific and Australian plates in this region is predominantly taken up by NE-SW trending strike-slip faults (Holt and Haines, 1995).

\section{Tectonic setting of central New Zealand}

Nearly two-thirds of the Marlborough region is underlain by greywacke of the Torlesse Supergroup, a laterally uniform structure (Figure 1.1; Mortimer, 2004). The Marlborough Fault System (MFS) marks the transition from a subduction plate boundary in 


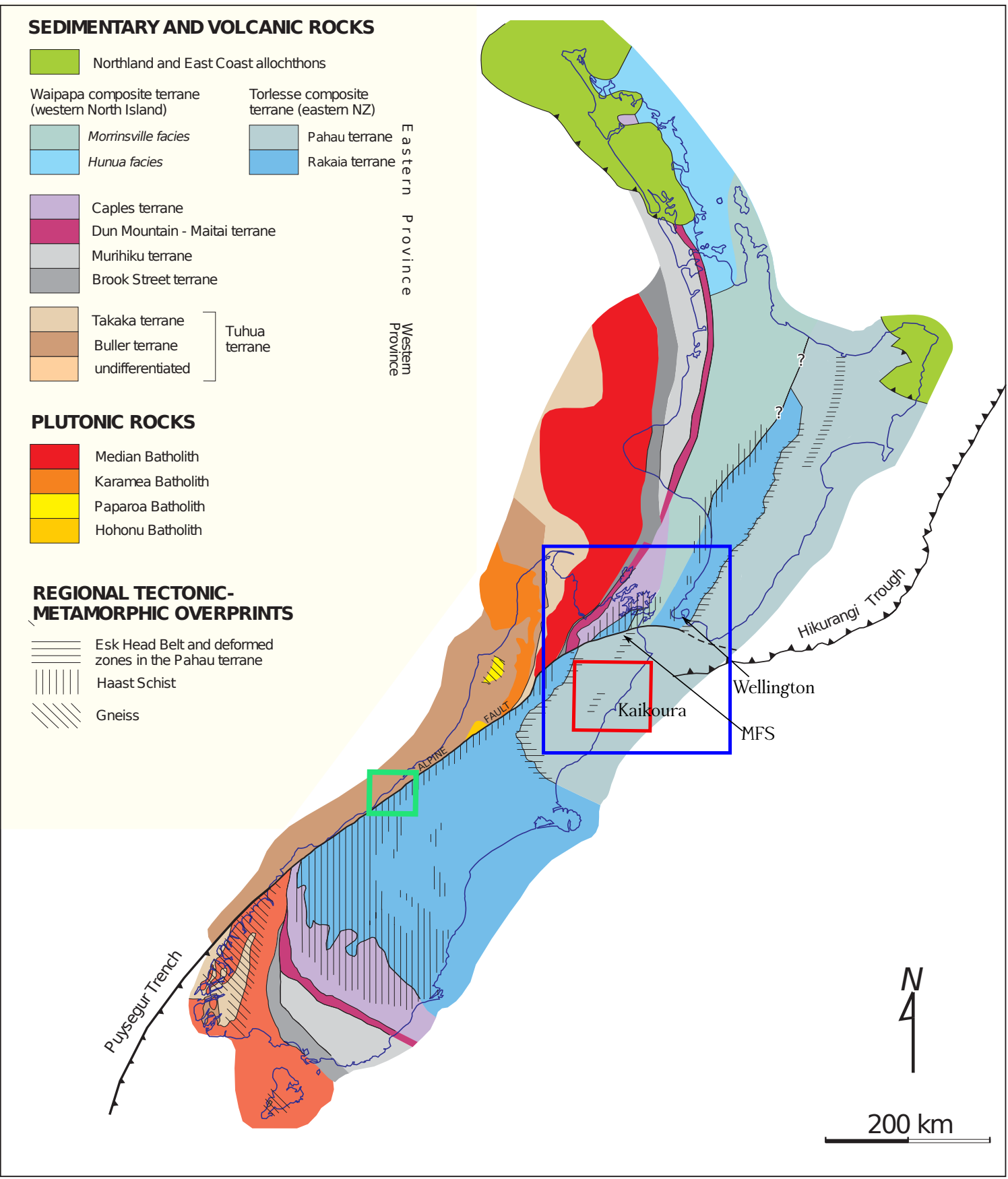

Figure 1.1: Basement (pre-late Cretaceous) geological map of New Zealand. The units are grouped according to major rock type. The blue, red and green boxes are the study area for the Kaikōura, simulation and the Whataroa study respectively. Figure adapted from Mortimer (2004) and referenced therein.

the north to a transpressive plate boundary (the Alpine Fault) in the south. The MFS may have developed as a series of "through-going" or a continuous single strike-slip structure, as each of the strike-slip faults is transported towards the NW (Van Dissen and Yeats, 1991; Anderson et al., 1993). The faults in the MFS are predominantly dextral strike-slip (trending NE-SW parallel to the strike of the Pacific-Australia plate 
motion, Figure 1.2), with a relatively small component of reverse motion (Van Dissen and Yeats, 1991). The average regional strike of the faults is $55^{\circ}$ and their near-surface dips vary from $60^{\circ}$ to near-vertical (Van Dissen and Yeats, 1991; Anderson et al., 1993).

Several of the major faults within the MFS, in particular the Awatere Fault (AF) (see Figure 1.2), are associated with near vertical low-velocity zones that extend to $\sim 23 \mathrm{~km}$ depth which have been interpreted as zones of high pore fluid pressure (Wannamaker et al., 2009; Eberhart-Phillips and Bannister, 2010). Slip rates in the range of 4 to $8 \mathrm{~mm} / \mathrm{yr}$ are observed along the Awatere (AF), Clarence (CF), and Wairau faults (WF) (see Figure 1.2) (Van Dissen and Yeats, 1991; Langridge et al., 2005; Wallace et al., 2007). The southernmost fault in the MFS, the Hope Fault (HF) (see Figure 1.2), is New Zealand's second fastest slipping onshore fault at rates of 13 to $23 \mathrm{~mm} / \mathrm{yr}$ (Langridge et al., 2005; Van Dissen and Yeats, 1991).

The tectonics around the Wellington region are dominated by the oblique subduction of the Pacific oceanic plate beneath the Australian plate. Major surface structural features around the Wellington region strike NE-SW with the active faulting also following this NE-SW trend (Mortimer, 2004; Litchfield et al., 2017). Also, the dominant geological features around the region are aligned sub-parallel to the strike of subduction. The predominant surface rocks are greywacke (Mortimer, 2004). The Wellington region lies above the forearc region of the Hikurangi subduction zone. This makes the study of anisotropic structure of the overlying crust feasible.

The Alpine Fault is an $\sim 850 \mathrm{~km}$ long, dextral-reverse fault that cuts across the west coast of New Zealand and it appears as a remarkably linear feature at a scale of tens of kilometers. There have been suggestions that the fault is locked and strain release is mainly coseismic due to no evidence of significant interseismic creep at shallow depths during the past 4 decades (Sutherland et al., 2007; Beavan et al., 2010). Several studies have suggested that the Alpine Fault is capable of generating $M_{w} \geq 8$ earthquakes with a recurrence interval of $291 \pm 23$ years and it is inferred to be late in its earthquake cycle (Sutherland, 1999; Sutherland et al., 2007; Howarth et al., 2015, 2018). The last rupture on the Alpine Fault, a $M_{w} 8.1 \pm 0.1$ that happened in $1717 \mathrm{AD}$, is inferred to have spanned about 300 to $500 \mathrm{~km}$ of the fault (Sutherland et al., 2007; Howarth et al., 


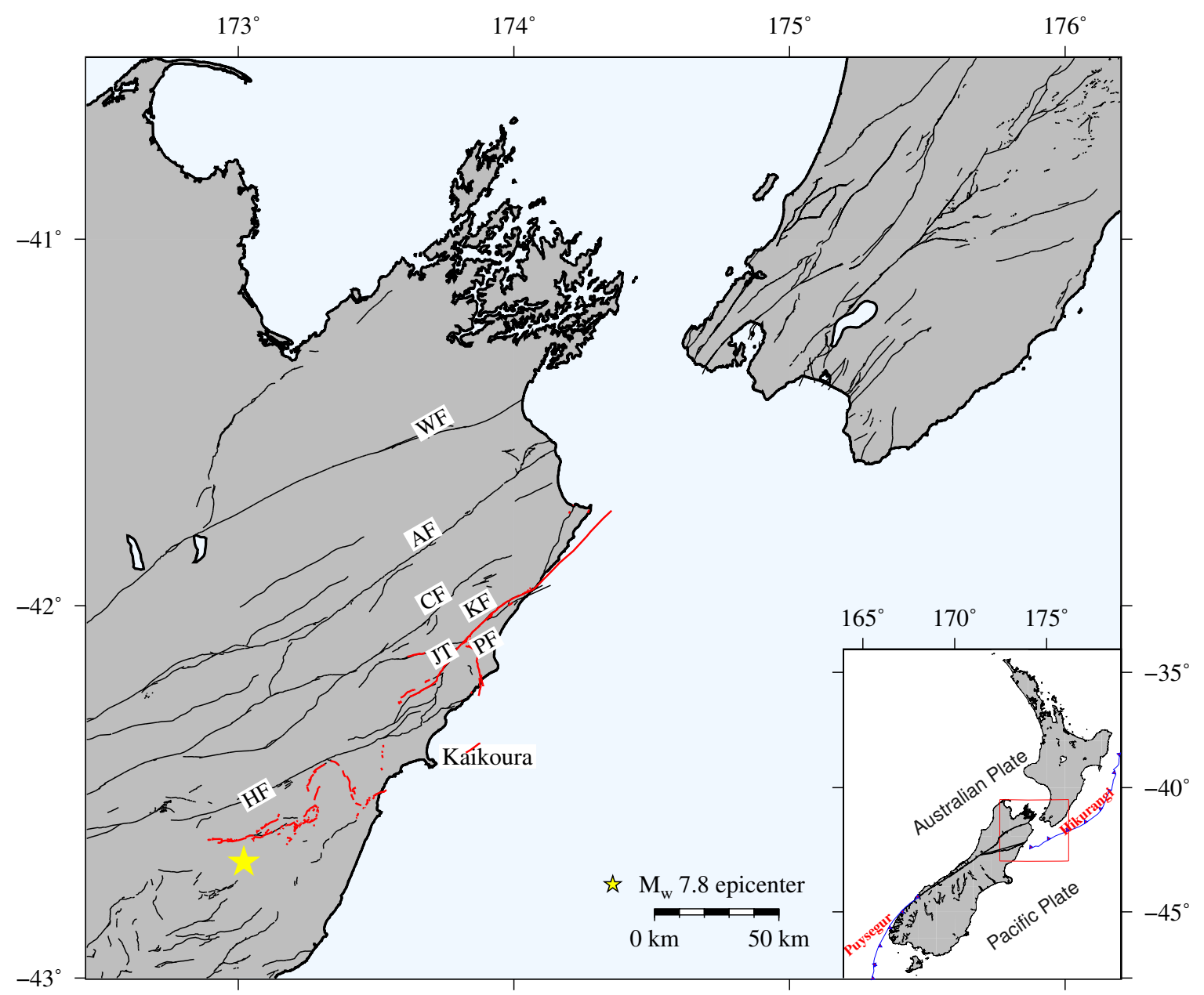

Figure 1.2: Map showing the tectonic setting of the Marlborough Fault System (MFS), New Zealand, with the main structural features associated with the AustralianPacific plate-boundary. Major faults marked include the Wairau (WF), Awatere (AF), Clarence $(\mathrm{CF})$ and Hope (HF) faults. The red lines denote the surface ruptured faults during Kaikōura mainshock; including the Jordan thrust (JT), Needles (NF), Papatea (PF) and Kekerengu (KF) faults (Langridge et al., 2016). The yellow star indicates the epicenter of the $M_{w} 7.8$ Kaikōura earthquake. Inset: The study region (red frame): the blue line is the plate boundary fault showing the Hikurangi and Puysegur trench.

\section{5, 2018).}

The Whataroa Valley is a former glacial valley deployed on unconsolidated glacial and river sediments that crosses the central segment of the Alpine Fault (green box on Figure 1.1). It is the junction between a strike-slip and thrust segment of the Alpine Fault (Norris and Cooper, 2001; Norris and Toy, 2014; Langridge et al., 2018). Around the valley, the Alpine Fault strikes at approximately $055^{\circ}$ as an oblique thrust fault (Little et al., 2005; Cox et al., 2008; Barth et al., 2012; Langridge et al., 2018). Phase two of the Deep Fault Drilling Project in the Whataroa valley revealed some of the 
extreme hydrothermal conditions in here, with a geothamal gradient of $125 \pm 55^{\circ} \mathrm{C} / \mathrm{km}$ and elevated pore fluid pressure (10\% above hydrostatic levels) (Sutherland et al., 2017; Townend et al., 2017; Toy et al., 2017). Sutherland et al. (2017) suggested that the extreme hydrothermal conditions were due to fluid flow within the fractured rocks and the exhumation of hotter rocks from depth.

\subsection{Seismic anisotropy and shear wave splitting}

This subsection introduces seismic anisotropy and shear wave splitting. We start with an overview of seismic anisotropy followed by types of anisotropic symmetry systems. We also introduce the governing equations of seismic wave propagation in an anisotropic medium. The section also introduces shear wave splitting, a feature of plane waves passing through anisotropic media that can be used to measure seismic anisotropy. We give a general overview of the phenomenon and a detailed review of its application to measure seismic anisotropy in various tectonic settings around the world.

\subsubsection{Seismic anisotropy}

An anisotropic material by definition, is a material that has varying physical properties depending on direction. This is in contrast to heterogeneous materials which have physical properties that depend on location. Whether a material is considered to be heterogeneous or anisotropic is often a matter of the scale at which we analyze its properties (Maupin and Park, 2007). Seismic anisotropy is the dependence of seismic velocity on wave propagation direction as caused by the elastic properties of rocks (Babuska and Cara, 1991). Anisotropy could be responsible for large variations in seismic velocities (Babuska and Cara, 1991; Anderson, 1989). Figure 1.3 illustrates how anisotropy can occur at different scales. Seismic anisotropy can provide valuable information on dynamic processes, such as the distribution of deformation in the crust (Koulakov et al., 2015), and is also a useful attribute for characterisation and detection of fabric orientations. It has been used as a measure for characterising crack density and orientation or crystal alignment (Crampin et al., 2015; Savage et al., 2016). It 
has been studied throughout the Earth, ranging in depth from hydrocarbon reservoirs in the shallow crust (Crampin and Peacock, 2008; Verdon et al., 2009; Bale et al., 2009) to the core and deep mantle (Wookey and Helffrich, 2008). Seismic anisotropy is often considered to be an indicator of stress orientation in the crust, because closure of cracks due to differential stress leads to (transverse) waves polarized parallel to the cracks traveling faster than the orthogonal direction (Crampin, 1984; Savage, 1999).

Alternatively, changes in pore fluid and cracks can cause variation in seismic anisotropy (Crampin, 1984; Savage, 1999). The measurements of changes in anisotropy has also been proposed as a possible technique for volcano eruption forecasting (Miller and Savage, 2001; Gerst and Savage, 2004; Savage et al., 2015), and as a stress forecasting tool (Gao and Crampin, 2015; Crampin et al., 2015).
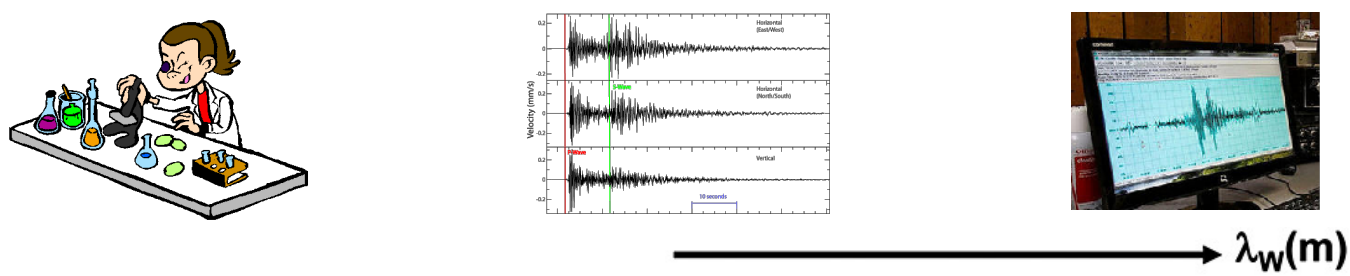

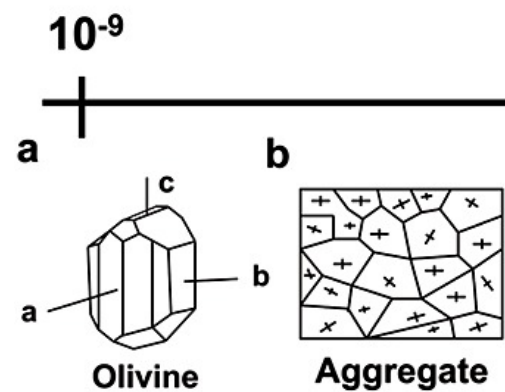

Micro

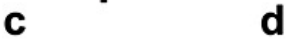

d

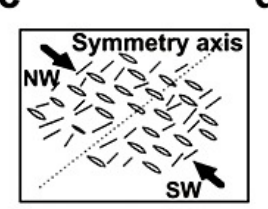

sw

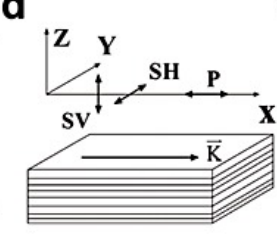

Intermediate
$10^{6}$

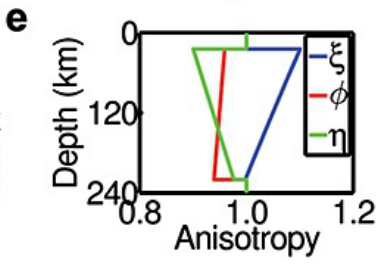

Macro

Figure 1.3: Seismic anisotropy on a scale of micro to macro. Micro scale: (a) The anisotropic crystals. (b) The anisotropic aggregate as an example of crystal preferred orientation. Intermediate scale: (c) and (d) Cracks filled model and finely layered model (example from sedimentary basin). Macro scale: (e) Seismic anisotropy observed at the lithosphere, asthenosphere boundary and the upper mantle. $\lambda_{s}$ and $\lambda_{s w}$ represent the wavelength. Modified from Wang et al. (2013)

\section{The theory of elasticity}

The theory of elasticity is the mathematical description seismologists use to describe the deformation (strain) of elastic materials in response to an applied force (stress). 
The applied deformation to a pure elastic system can be recovered when the acting force is removed. But in rock physics, where materials are often described to behave in a viscoelastic manner, the strain induced by an applied stress is not completely elastically recoverable.

Rock anisotropy is a common phenomenon in the Earth's crust caused by an array of mechanisms, such as sequences of fine layers, stress-induced crack alignments, and fracture fabric in crustal rocks. Assuming we can describe the anisotropic rock as a homogeneous and continuous linear-elastic medium, we can obtain a general constitutive relationship between stress, $\sigma$ and strain, $\epsilon$ (Equation1.1, using the summation convention) by following Hooke's law (Babuska and Cara, 1991; Stein and Wysession, 2003; Li, 2016).

$$
\sigma_{i j}=c_{i j k l} \epsilon_{k l}
$$

To describe a nonlinear-elastic medium, a quadratic term needs to be added to the right side of Equation1.1. Here $\sigma_{i j}$ is the stress tensor, $\epsilon_{k l}$ is the strain tensor and $c_{i j k l}$ is the stiffness (or elasticity) tensor. The stress tensor and strain tensor are second-order symmetric tensors. Mathematically, the stiffness tensor can be used to describe the degree of order within a medium. The stiffness tensor is a fourth order tensor with 81 elements $\left(3^{4}\right)$ which can be reduced to 36 because of the symmetry between the stress and strain tensors. These 36 coefficients (or constants) for an arbitrary medium are often arranged in a $6 \times 6$ matrix called the stiffness matrix $c_{i j}$ (Equation1.2). Due to the symmetry of stress, strain and stress tensors $\left(c_{i j k l}=c_{j i k l}=c_{i j l k}=c_{j i l k}, c_{i j k l}=c_{k l i j}\right.$, $\sigma_{i j}=\sigma_{j i}$ and $\epsilon_{k l}=\epsilon_{l k}$ ) the 36 elements (Equation1.2) can be reduced to 21 independent 
elements (Equation1.3).

$$
\begin{aligned}
& c_{i j k l}=\left[\begin{array}{cccccc}
c_{1111} & c_{1122} & c_{1133} & c_{1123} & c_{1113} & c_{1112} \\
c_{2211} & c_{2222} & c_{2233} & c_{2223} & c_{2213} & c_{2212} \\
c_{3311} & c_{3322} & c_{3333} & c_{3323} & c_{3313} & c_{3312} \\
c_{2311} & c_{2322} & c_{2333} & c_{2323} & c_{2313} & c_{2312} \\
c_{1311} & c_{1322} & c_{1333} & c_{1323} & c_{1313} & c_{1312} \\
c_{1211} & c_{1222} & c_{1233} & c_{1223} & c_{1213} & c_{1212}
\end{array}\right] \\
& c_{i j k l}=\left[\begin{array}{cccccc}
c_{1111} & c_{1122} & c_{1133} & c_{1123} & c_{1113} & c_{1112} \\
& c_{2222} & c_{2233} & c_{2223} & c_{2213} & c_{2212} \\
& & c_{3333} & c_{3323} & c_{3313} & c_{3312} \\
& & & c_{2323} & c_{2313} & c_{2312} \\
& & & & c_{1313} & c_{1312} \\
& & & & & c_{1212}
\end{array}\right]
\end{aligned}
$$

However, for simplicity of notation we introduce the Voigt matrix notation to reduce the number of subscripts of the stiffness tensor to two. This Voigt matrix notation consists of replacing ij or $k l$ by $p$ or $q$ as shown in Table 1.1. This enables the simplified comparison, visualization and manipulation of fourth-order tensors. In a two-index notation, the stiffness tensor is represented in Equation1.4 such that $c_{11}=c_{1111}$ and $c_{14}=c_{1123}$.

$$
c_{p q}=\left[\begin{array}{cccccc}
c_{11} & c_{12} & c_{13} & c_{14} & c_{15} & c_{16} \\
& c_{22} & c_{23} & c_{24} & c_{25} & c_{26} \\
& & c_{33} & c_{34} & c_{35} & c_{36} \\
& & & c_{44} & c_{45} & c_{46} \\
& & & & c_{55} & c_{56} \\
& & & & & c_{66}
\end{array}\right]
$$

The 21 independent elastic coefficients can often be reduced to describe an elastic system of a generic anisotropic rock. In fact, the anisotropic symmetry systems of most 
Table 1.1: Conversion from tensor to matrix indices using the Voigt notation

\begin{tabular}{ll}
\hline$i j$ or $k l$ & $p$ or $q$ \\
\hline 11 & 1 \\
22 & 2 \\
33 & 3 \\
23 or 32 & 4 \\
31 or 13 & 5 \\
21 or 12 & 6 \\
\hline
\end{tabular}

Table 1.2: Classification of anisotropic media on the basis of the number of planes of symmetry and independent elastic coefficients. The number of planes of symmetry obtained from Chadwick et al. (2001)

\begin{tabular}{llll}
\hline $\begin{array}{l}\text { Type of sym- } \\
\text { metry }\end{array}$ & Typical mineral & $\begin{array}{l}\text { Number of planes } \\
\text { of symmetry }\end{array}$ & $\begin{array}{l}\text { Number of in- } \\
\text { dependent elas- } \\
\text { tic coefficients }\end{array}$ \\
\hline Triclinic & Plagioclase & 0 & 21 \\
Monoclinic & Hornblende & 1 & 13 \\
Orthorhombic & Olivine & 3 & 9 \\
Tetragonal & Stishovite & 5 & 6 \\
Hexagonal & Ice & 7 & 5 \\
Cubic & Garnet & 9 & 3 \\
Isotropy & Volcanic glass & $\infty$ & 2 \\
\hline
\end{tabular}

common Earth materials have fewer than 21 elastic coefficients (Crampin, 1984). Such symmetry systems include the triclinic with 21 , the monoclinic with 13 , orthorhombic with 9 , tetragonal with 6 , hexagonal with 5 , and cubic with 3 independent elastic constants (Babuska and Cara, 1991; Stein and Wysession, 2003; Li, 2016). The number of planes of symmetry and independent elastic coefficients for types of symmetry system is given in Table 1.2 .

\section{Wave Equationin anisotropic medium}

Here we describe the propagation of plane waves in anisotropic media. We will start with a simple plane wave in an isotropic medium and move to waves in an anisotropic medium. Derivation and description of the wave Equationfollows Babuska and Cara (1991), Stein and Wysession (2003) and Li (2016). By considering Newton's laws of motion, the wave Equationof a particle in a continuous medium can be described as:

$$
\rho \frac{\partial^{2} u_{i}}{\partial t^{2}}=\frac{\partial \sigma_{i j}}{\partial x_{j}}+f_{i}
$$


where $u_{i}$ is the component of displacement, $t$ is time, $x_{j}$ is position, $f_{i}$ is the external force per unit volume, $\rho$ the density and $\sigma_{i j}$ is the stress tensor as described above. In a homogeneous anisotropic medium, if $f_{i}$ is set to zero, Equation1.5 becomes:

$$
\rho \frac{\partial^{2} u_{i}}{\partial t^{2}}=\frac{\partial \sigma_{i j}}{\partial x_{j}}
$$

The Equationfor plane wave displacement, $u$, at time $t$ and location $x$ can be expressed as:

$$
u(\mathbf{x}, t)=A e^{i \omega\left(t-\boldsymbol{\Lambda}_{c} \cdot \mathbf{x}\right)}
$$

where $A$ denotes the wave amplitude and polarity, and $\omega$ is angular frequency. $\boldsymbol{\Lambda}_{c}$ is the wavenumber vector that defines the direction of ray propagation and satisfies the condition that $\left|\boldsymbol{\Lambda}_{c}\right|=\omega / c\left(\left|\boldsymbol{\Lambda}_{c}\right|\right.$ denotes the magnitude). $c$ is the phase velocity of the medium. The second derivatives of Equation1.7 with respect to time $(t)$ and space $(\mathbf{x})$ give:

$$
\begin{gathered}
\frac{\partial^{2} u_{i}}{\partial t^{2}}=A_{i}(i \omega)^{2} e^{i \omega\left(t-\boldsymbol{\Lambda}_{c} \cdot \mathbf{x}\right)} \\
\frac{\partial^{2} u_{l}}{\partial x_{j} \partial x_{k}}=A_{l} \boldsymbol{\Lambda}_{c j} \boldsymbol{\Lambda}_{c k} e^{i \omega\left(t-\boldsymbol{\Lambda}_{c} \cdot \mathbf{x}\right)}
\end{gathered}
$$

where $\boldsymbol{\Lambda}_{c j}$ and $\boldsymbol{\Lambda}_{c k}$ denote the coefficient of the second derivative of Equation1.7 with respect to space. The relation between the second order strain tensor and displacement (Babuska and Cara, 1991; Stein and Wysession, 2003; Li, 2016) is expressed as:

$$
\epsilon_{k l}=\frac{1}{2}\left(\frac{\partial u_{k}}{\partial x_{l}}+\frac{\partial u_{l}}{\partial x_{k}}\right)
$$

Due to the inherent symmetry of the strain tensor, $\frac{\partial u_{k}}{\partial x_{l}}=\frac{\partial u_{l}}{\partial x_{k}},(k=l)$, and thus $\epsilon_{k l}=\frac{\partial u_{k}}{\partial x_{l}}=\frac{\partial u_{l}}{\partial x_{k}}$. Hooke's law, Equation1.1, can then be rewritten as

$$
\sigma_{i j}=c_{i j k l} \frac{\partial u_{l}}{\partial x_{k}}
$$


We obtain the linear wave Equationby substituting Equation1.11 into Equation1.6 and assuming that the stiffness coefficients are constant.

$$
\rho \frac{\partial^{2} u_{i}}{\partial t^{2}}=c_{i j k l} \frac{\partial^{2} u_{l}}{\partial x_{j} \partial x_{k}}
$$

Substituting equations 1.8 and 1.9 into Equation1.12 and dividing by the common terms, gives:

$$
\rho A_{i}=c_{i j k l} A_{l} \boldsymbol{\Lambda}_{c j} \boldsymbol{\Lambda}_{c k}
$$

which can be rewritten as

$$
c_{i j k l} A_{l} \boldsymbol{\Lambda}_{c j} \boldsymbol{\Lambda}_{c k}-\rho A_{i}=0
$$

We can rearrange Equation1.14 to put it in terms of the phase velocity, $c$, and ray propagation, $\vec{n}$, given that $\left|\boldsymbol{\Lambda}_{c}\right|=\omega / c$.

$$
\frac{1}{\rho} c_{i j k l} \boldsymbol{\Lambda}_{j} \boldsymbol{\Lambda}_{k} A_{l}-c^{2} A_{i}=0
$$

The first term on the left side of Equation1.15 can be expressed as a matrix called the Christoffel matrix, $m_{i j}$ :

$$
m_{i j}=\frac{1}{\rho} c_{i j k l} \boldsymbol{\Lambda}_{j} \boldsymbol{\Lambda}_{k}
$$

thus Equation1.15 can be rewritten as:

$$
m_{i j} A_{l}=c^{2} A_{i}
$$

Equation 1.17 is an eigenvalue problem which is satisfied by the three mutually orthogonal eigenvectors $\overrightarrow{A_{1}}, \overrightarrow{A_{2}}$ and $\overrightarrow{A_{3}}$ and their corresponding eigenvalues $c_{1}^{2}, c_{2}^{2}, c_{3}^{2}$. Solving for the three eigenvalues and their associated eigenvectors gives the value and orientation of the resultant quasi-P-wave, fast S-wave and slow S-wave (Babuska and Cara, 1991; Silver and Chan, 1991; Savage, 1999). 


\subsubsection{Shear wave splitting}

Shear wave splitting (SWS) is a common tool for measuring seismic anisotropy. In crustal geophysics, anisotropy in wave propagation may be generated by cracks that are closed if oriented perpendicular to the stress, and open if parallel (thus generating a differential in wave speeds in different directions). SWS occurs when a shear wave passes through a preferentially oriented (anisotropic) medium. When a shear wave enters an anisotropic medium, the incident shear wave splits into two polarized shear waves; a fast wave, oriented parallel to the open cracks, and a slow wave perpendicular to the crack. SWS is often characterized by two parameters, the fast direction $(\phi)$ and the delay time $(\delta t)$ as shown in Figure 1.4. The fast polarization direction estimated from shear waves propagating in a fractured medium, can be used to infer the fracture orientation and the time lag, $\delta t$, between the fast and slow shear-waves is associated with the fracture intensity or density. Boness and Zoback (2006) suggested that with knowledge of the formation properties, it is possible to identify stress-induced anisotropy, and accordingly determine the direction of the maximum horizontal compressive stress, $S_{H \max }$. In estimating SWS parameters $(\phi$ and $\delta t)$, we attempt to find the best splitting parameters that will "un-split" an anisotropic S-wave (correcting the seismograms for anisotropy). There are several methods used in estimating SWS parameters. These methods include the following: (1) transverse minimisation method (Silver and Chan, 1991), (2) eigenvalue method (Silver and Chan, 1991; Teanby et al., 2004a,b; Wessel, 2010), (3) cross correlation method (Vecsey et al., 2008), (4) splitting intensity method (Chevrot, 2000; Monteiller and Chevrot, 2010), and (5) cross convolution method (Menke and Levin, 2003). These techniques are applied to a data set consisting of three seismogram components from a single station. For this thesis, we used the eigenvalue method to estimate the SWS parameters. A detailed description of the method is presented in Section 2.2.1.

In addition to SWS techniques, there are other methods that can be used to measure anisotropy of a medium. Anisotropy can be measured using receiver function methods (Park, 1996), Love- and Rayleigh waves (Anderson, 1989) and P-wave delay 


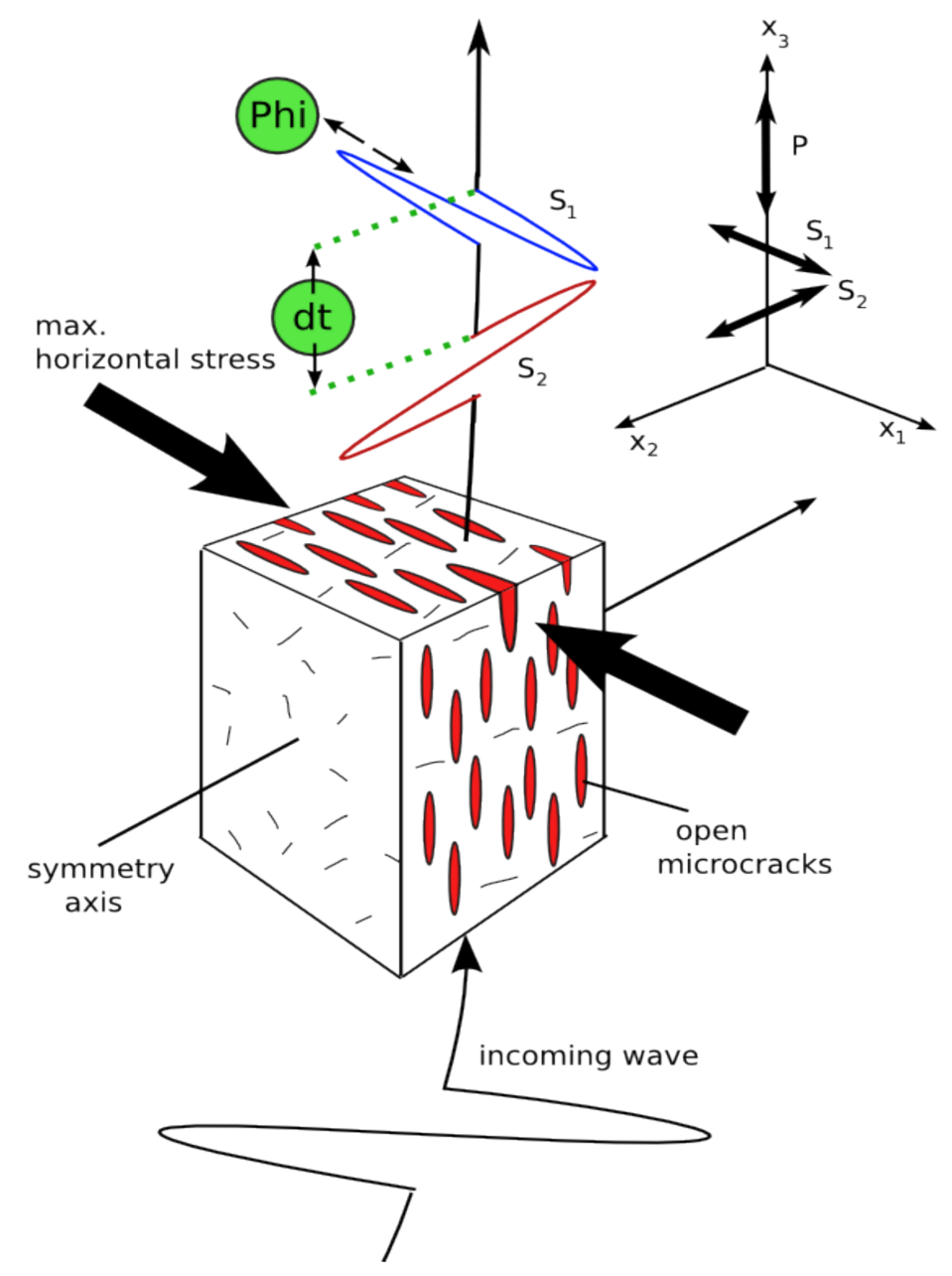

Figure 1.4: Crack-induced SWS: An incident shear wave (incoming wave) splits into two polarized waves upon encountering the anisotropic medium (box). The blue wave, S1 (fast-quasi shear) wave travels faster than the red wave, S2 (slow-quasi shear). We often describe the split shear waves in terms of two parameters, the delay time, $\delta t$ (the delay time between S1 and S2) and the fast direction, $\phi$, which is the polarization direction of the fast-quasi shear wave. Cracks that are not aligned parallel to the maximum stress tend to close, which leads to anisotropy with fast direction parallel to the orientation of maximum horizontal stress. The width of crack-openings are exaggerated. From Wessel (2010) and Walsh (2012).

times (Babuska and Cara, 1991). Fouch and Rondenay (2006) give a concise discussion of other methods and comparison of techniques used in measuring seismic anisotropy. The use of surface waves for measuring seismic anisotropy produces a better vertical resolution and poorer lateral resolution compared to the SWS method (Fouch and Rondenay, 2006; Fry et al., 2014). However, to delineate the spatial extent of seismic anisotropy (in the shallow crust) and to search for temporal changes in crack induced anisotropy, the SWS technique seems to be the most suitable (Crampin et al., 1999; 
Savage, 1999; Silver and Chan, 1991; Crampin et al., 2015). This is because compared with other waves, shear waves velocities are largely affected by fluid-filled cracks (Crampin, 1984).

\subsubsection{Measuring crustal anisotropy}

Seismic anisotropy of the upper crust has been studied extensively, mostly based on analyzing SWS from local or regional earthquakes (e.g. Crampin and Booth, 1989; Crampin et al., 2015). Anisotropy in the crust can be produced by multiple physical processes at different spatial scales. In the crystalline upper crust, anisotropy can be attributed to two broad mechanisms: stress-related anisotropy and structural anisotropy (see Figure 1.5; Boness and Zoback, 2006). In the Earth's crust, anisotropy may be caused by preferentially aligned joints or micro-cracks, by layered bedding in sedimentary formations, or by highly foliated metamorphic rocks. Crustal anisotropy observed within orogenic belts and active plate boundaries is known to be influenced by tectonic activity within the crust (Okaya et al., 2016). Anisotropy in the upper crust is usually analyzed in relation to the opening of cracks by the tectonic stresses of the area (Maupin and Park, 2007). Thus, the anisotropic behaviour in the Earth's crust is presumed to originate from one of four known mechanisms (Boness and Zoback, 2006; Crampin, 1985) : (1) microcracks opening in response to stress (stress controlled anisotropy); (2) rock texture associated with crystal or mineral alignment, (3) lithologic fabrics (e.g., aligned grains); and (4) structural properties (e.g., sedimentary layering, large-scale faults).

The presence of shear or metamorphic foliations in fault zones and metamorphic terranes can serve as proxies for intra-crustal deformation (Godfrey et al., 2000). Crustal anisotropy originating from aligned cracks can be used to determine the state of stress in the crust, since in many cases open cracks are preferentially aligned with orientation of the maximum horizontal compressive stress (Crampin et al., 2015; Balfour et al., $2012,2005)$. Structures such as cracks or fractures in the vicinity of active faults normally have their orientations parallel to the fault plane (Godfrey et al., 2000). In active tectonic areas, such as near faults and volcanoes, crustal anisotropy can be used to look 


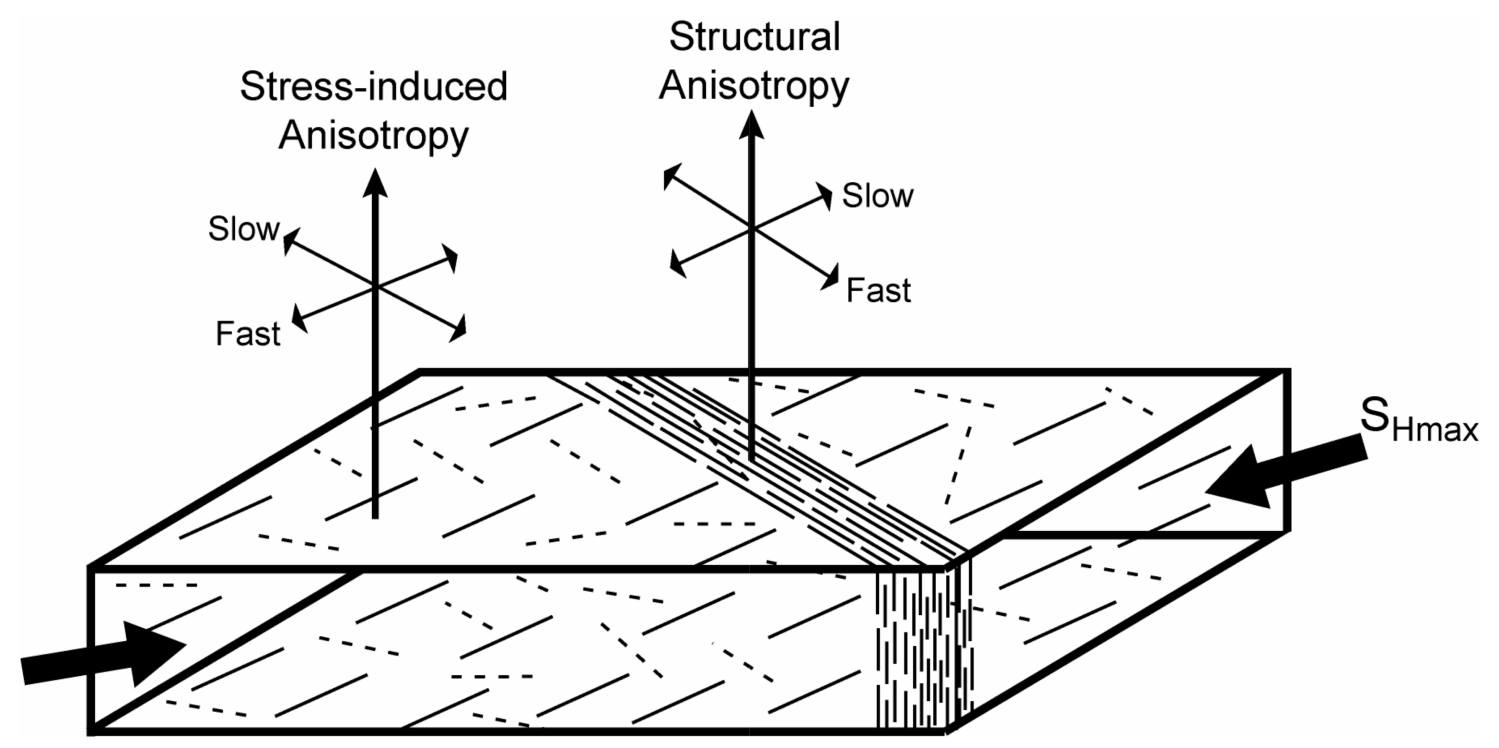

Figure 1.5: Illustrating of stress induced and structural controlled anisotropy. The figure shows stress-induced adjacent to structurally controlled crustal anisotropy. Here vertical propagating shear waves are polarized with a fast direction parallel to $S_{H \max }$ due to the preferred orientation created by closed fracture/cracks (dashed lines). Vertical shear waves propagating inside the fault zone tend to have the fast direction parallel to the strike of the fault fabric. Figure from Boness and Zoback (2006).

for changes in preferred orientation of cracks that may indicate a rotation of the stress field (Godfrey et al., 2000).

SWS of local earthquakes has been used to monitor how anisotropy changes spatially in the vicinity of major fault systems (Peng and Ben-Zion, 2004; Cochran et al., 2006; Maupin and Park, 2007). However, studies have also shown anisotropy can be complicated by crustal structure (Balfour et al., 2005; Boness and Zoback, 2006; Balfour et al., 2012). To use seismic anisotropy as a stress indicator requires identifying which areas in the crust are influenced by stress or structures. The source of anisotropy in a region can be interpreted in light of the maximum horizontal compressive stress orientation (determined from earthquake focal mechanism inversion) and the orientation of structures. There have been reviews and debates over whether precursory loading of earthquake fault zones can be detected by temporal changes in crack anisotropy ( Qinya et al., 2004; Peng and Ben-Zion, 2005; Crampin et al., 2015). Determining the sources of variability in anisotropy can lead to a better understanding of the crustal structure and stresses, and in the future may be used as a stress monitoring and mapping tool 
(Miller and Savage, 2001; Gerst and Savage, 2004; Balfour et al., 2005; Savage et al., 2015; Crampin et al., 2015).

\subsubsection{Variations in measuring seismic anisotropy using shear wave splitting}

Observations of crack-induced seismic anisotropy can provide insights into crustal stresses (Crampin et al., 2015; Savage et al., 2015). Cracks oriented normal to principal stress directions are closed while cracks parallel to the stress may be opened (Crampin, 1985, 1988). Therefore cracks tend to align parallel to the maximum stress direction; hence changes in the orientation of the principal stress direction can vary when cracks are opening or closing, thus changing the fast orientation. Also, changes in the magnitude of stress or pore pressure can affect the crack density and aspect ratio, and hence variations in delay times (Crampin et al., 2015; Savage et al., 2015). Therefore, observing changes in delay times and fast orientation over time could be used to infer changes in stresses around hazardous regions (Teanby et al., 2004b). Some researchers suggest that the accumulation of stress before earthquakes can be monitored by measuring variations in SWS parameters (Crampin and Booth, 1989; Crampin and Zatsepin, 1997; Volti and Crampin, 2003a,b; Gao and Crampin, 2004). These variations modify the microcrack geometry, which can be monitored through SWS (Crampin, 1994). Temporal variations of SWS measurements may yield insight into changes in stress around hazardous regions (Miller and Savage, 2001; Gerst and Savage, 2004; Crampin et al., 2015; Savage et al., 2015). However, other researchers have refuted these suggestions (e.g. Aster et al., 1990). Aster et al. (1990) argued that the observed "temporal" variations by Crampin and Booth (1989) are likely due to spatial variation and/or heterogeneity in the crust (serving as scatterers that contaminate the recorded waveforms). Stress variations are typically concentrated at the margins of tectonic plates (Turcotte, 1982).

Splitting measurements can also be used to detect subtle in changes $S_{H \max }$ caused by tidal effects (e.g. Teanby et al., 2004b). Consistency of ground deformation inferred from GPS measurements and delay time changes has been previously observed (e.g. 
Savage et al., 2015). Temporal changes of hydrothermal activity and gas release are also considered as a cause of splitting changes in volcanic areas (e.g. Gerst and Savage, 2004; Roman et al., 2011; Unglert et al., 2011; Johnson et al., 2015). Analysis of regional stresses and the distribution of anisotropy around a volcanic area (Soufriere Hills Volcano, Montserrat), coupled with fault plane solution analyses, succeeded in explaining precursory stress change caused by diking (e.g. Roman et al., 2011).

There have been many studies on the relevance of measuring seismic anisotropy. Roman et al. (2011) observed rotations of fast directions that correlated with rotating fault plane solutions associated with volcanic activity at Soufriere Hills volcano in Montserrat. Savage et al. (2010b) also observed strong correlations between shear wave splitting parameters and GPS baseline length changes at Asama volcano in Japan. Miller and Savage (2001), observed changes in fast orientation before and after an eruption at Mt. Ruapehu volcano, New Zealand. They interpreted this as a variation in principal stress direction which they related to an increase in pore pressure. They further recommended that the shear wave splitting technique could be used as an additional tool for volcano monitoring. Zheng et al. (2008) also studied the temporal variations of shear wave splitting in the aftershock region of 1999 Chichi Earthquake, Taiwan. They observed a decrease in delay times shortly before the Chichi main shock and two Chiayi earthquakes. They went on to suggest that the temporal variations of shear-wave splitting are of great significance, since they provide information about the stresses around the region which can serve as critical warning information before large earthquakes (Zheng et al., 2008). Bianco et al. (2006) also observed variations in fast orientation and delay times before the 2001 eruption on Mt Etna. They observed SWS time delays exhibiting a sudden decrease shortly before the start of the eruption and a change in the fast direction five days before the onset of the eruption (Bianco et al., 2006).

Liu et al. (1997) showed that through measuring seismic anisotropy it is possible to obtain information about strain (deformation) in the Earth's crust. They suggested that the build-up of stress before earthquakes may be monitored and interpreted by the analysis of shear wave splitting. Moreover, Crampin (1990a) reported to have 
"successfully" stress-forecast the time and magnitude of a M 5 earthquake in southwest Iceland three days before it occurred. The changes in shear wave splitting from small events before and after a large earthquake in California led to the suggestion that such changes could be used to predict earthquakes (Crampin, 1990a; Crampin and Gao, 2013; Crampin et al., 2015). With all these successes, measuring temporal changes in stress indicators through SWS would be a useful tool in hazard studies. However, the interpretations are sometime difficult and subjective.

\subsubsection{Variation in SWS measurements}

There have been several studies and well-documented instances of variations in measured splitting parameters, particularly delay times over time (Johnson et al., 2010; Walsh et al., 2013; Crampin et al., 2015; Savage et al., 2015). These variations are sometimes attributed to the changes in source locations leading to diverse of ray paths (Johnson et al., 2010; Walsh, 2012). Other possible causes are: multiple anisotropic layers; dipping symmetry axes; lateral variations in anisotropy; scattering of seismic energy by topography and; heterogeneity of the Earth and other sources of noise ( Walsh et al., 2013; Savage et al., 2015). These factors can introduce variations in the splitting parameters, hence affecting the accurate measurement of SWS parameters. Castellazzi et al. (2015) also suggested the effect of scattering on seismic waveforms as a plausible explanation for the variation of SWS measurements.

Aster et al. (1990) suggested that the inability to identify a distinct slow quasi-shear wave pulse can be an indication that scattering, strongly affects the particle motion (Aster et al., 1990). Johnson et al. (2010) observed abrupt changes in delay times which they initially attributed to cycle skipping (a phenomenon caused by misfitting peaks of a waveform with different peaks or a trough; see Figure 1.6) but further multiplet waveform analysis around the Okmok volcano revealed a possibility of contamination of shear waves by scattered seismic energy. They suggested these scatterers (due to heterogeneity in the medium) can contaminate the shear wave coda, and so inhibit the detection of any subtle changes in shear wave splitting parameters. Examination of waveform coda of clustered earthquakes at Mount Ruapehu by Johnson et al. (2011), 
revealed systematic changes that were interpreted as being caused by seismic scatterers. Similar behaviour was observed when earthquake multiplet records around Piton de la Fournaise volcano were examined (Walsh et al., 2013; Savage et al., 2015).

Baisch (2001) investigated the spatial and temporal changes in seismic wave character associated with the 1989 Loma Prieta main shock. Using a moving window technique to compute inter station coherency depending on time and frequency of seismogram pairs, they observed reduced coherencies with post- and pre- Loma Prieta local events (in a region where closely spaced events to the mainshock hypocenter are located). They suggested that cracks opened co-seismically, causing the wavefield to scatter after the Loma Prieta event. Also, the post-seismic relaxation processes such as crack healing, fluid diffusion, and deformations lead to the progressive closure of these cracks with time, resulting in changes in seismic wave character. Thus, the effect of scattering cannot be ignored in crustal studies (Dainty, 1996; Baisch, 2001; Sato et al., 2012).
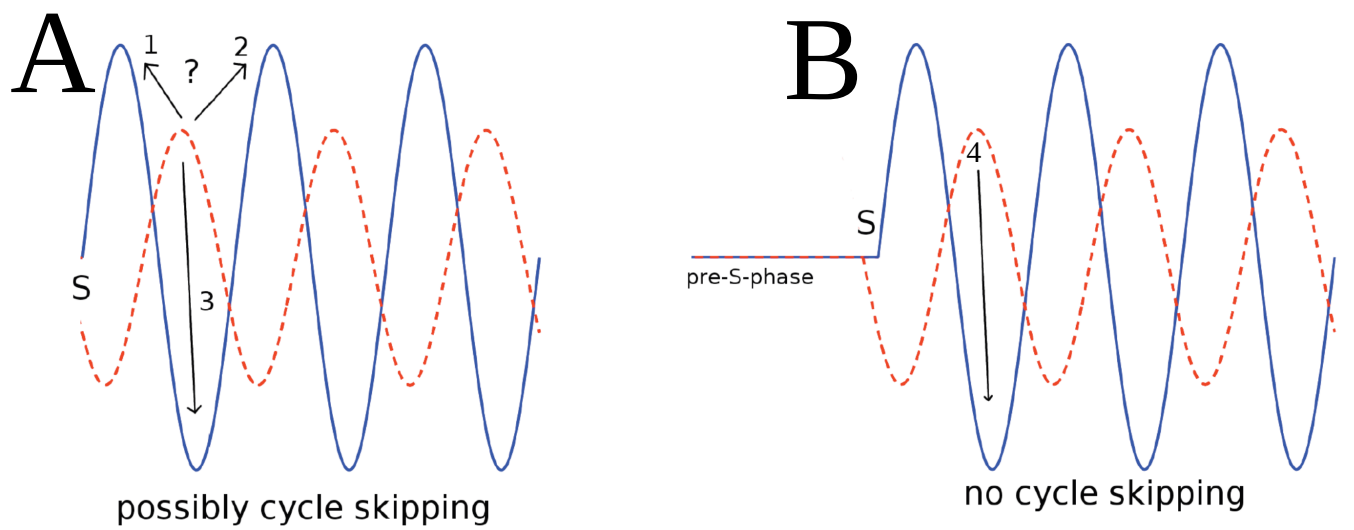

Figure 1.6: We consider SWS measurements to suffer from cycle skipping, if the waveforms (red and blue on A) can be matched in several ways (arrows 1,2 and 3 on A). Often, the uncertainty of the delay time for such measurement is a multiple of half a period length. However, if parts of the seismogram before the S-wave arrival are included (B), the waveforms can only be matched in one way (arrow 4 on B) (Wessel, 2010). In such a case, the measurements are not affected by cycle skipping. Figure from Wessel (2010) 


\section{Fault healing}

Most studies of fault zone strengthening or healing after an earthquake have been based on laboratory experiments (e.g. Dieterich, 1972; McLaskey et al., 2012; Aben et al., 2017). More recently, efforts have focused on trying to relate measured changes in the seismic properties in fault zones to fault healing after large earthquakes (e.g. $\mathrm{Li}$ et al., 1998; Tadokoro et al., 1999; Tadokoro and Ando, 2002; Tadokoro et al., 2002). Dieterich (1972) studied fault healing as problem of temporal change in the coefficient of strength of friction through laboratory experiments. He observed that the coefficient of static friction of surfaces with a gouge exhibits a highly time-dependent behavior. He suggested that this behavior may be important in understanding the mechanisms of earthquake foreshocks, aftershocks, and fault creep (Dieterich, 1972). Aben et al. (2017) through a suite of laboratory experiments, tried to understand the postseismic recovery within fault damage zones and how healing of coseismic fractures happens over time. After 64 days (after fracturing) of subjecting quartz-monzonite samples to upper crustal conditions, they observed $50 \%$ recovery, of the initial drop in $\mathrm{P}$ wave velocity and an unchanging permeability. They demonstrated that the $\mathrm{P}$ wave velocity recovery could be associated with calcite sealing and asperity dissolution (Aben et al., 2017). They concluded that fault damage zones could cause fluid conduits to remain open after an earthquake for longer durations than suggested by the seismic monitoring of fault healing (Aben et al., 2017).

Li et al. (1998) used direct observations to detect evidence for fault healing from increases in seismic velocities between two identical active source seismic experiments at the Johnson Valley fault, near Parkfield in California. They concluded that cracks opening during large earthquakes closed soon after ( $\sim 2$ to 4 years after the earthquake). Closure of cracks would increase the frictional strength of the fault zone, as well as it's stiffness. Thus, such a pattern of healing fault zones may help explain observations of increasing stress drops together with increasing recurrence intervals of repeating micro earthquakes ( $\mathrm{Li}$ et al., 1998). A comparison of earthquakes before and after a specific large event showed a small coseismic reduction in wave velocity at stations with 
unconsolidated sedimentary rocks that had been strongly shaken (Dodge and Beroza, 1997). Dodge and Beroza (1997) suggested that temporal changes were a shallow effect of shaking, rather than a physical change in the bedrock. They concluded that the velocity decrease was more likely caused by crack opening or cracks coalescing due to strong shaking from the mainshock (Dodge and Beroza, 1997).

Observations of fault healing through SWS measurements have been previously reported (e.g. Tadokoro et al., 1999; Tadokoro and Ando, 2002; Tadokoro et al., 2002). Tadokoro et al. (1999) proposed a hypothesis of detecting and monitoring fault healing by means of repeated SWS analyses. They analyzed SWS using the aftershocks of the 1995 Hyogoken Nanbu earthquake $\left(\mathrm{M}_{w}\right.$ 6.9) (12 months after the mainshock) and observed that the fast direction at stations on fault zones were roughly parallel to the fault strike, which was perpendicular to the tectonic stress field. Based on the assumption that the fault-origin shear fracture is vertical, (because the dip angle of the Nojima fault is roughly vertical) they concluded that a fault-parallel fast direction is caused by new fractures generated by the shear faulting during the main-shock.

\section{Temporal variations of shear wave splitting in the crust}

The temporal variation of shear wave splitting and its interpretation is highly controversial (Aster et al., 1990; Crampin, 1990a; Crampin et al., 2015, e.g.). One of the main problems with the interpretation of temporal changes is the possibility of spatial variations being sampled along differing ray-paths at different times and being incorrectly interpreted as temporal changes. To resolve these controversies and ambiguity, there is a need to understand where the anisotropy is acting, so that even if the ray-paths differ, changes can be inferred. Also, keeping the ray-path constant can ensure that, even if the exact location of the anisotropy is not known any changes observed could be confidently interpreted to be temporal. The use of multiplets has the potential for detection of temporal changes in anisotropy. 


\section{Data and methods}

This thesis relies on the analysis of both observed (Chapters 3 and 4) and simulated waveforms (Chapter 5). This Chapter describes a number of methods and data used for both empirical and simulation studies. We start with a brief description of the GeoNet earthquake catalog and the waveforms used. We follow this with a description of the method used to estimate shear wave splitting (SWS) parameters. Finally, we describe the Spectral Element Method (SEM) used to generate simulated synthetic waveforms used in Chapter 5.

\subsection{Data}

\subsubsection{Seismic network}

The seismic data used in Chapters 3 and 4 are discussed in detail in each Chapter. Here, we give a brief introduction to the data used. For the anisotropic study around central New Zealand, we used 36 stations deployed around the Wellington and Marlborough regions. These stations include 24 permanent, 3 component seismic stations (a combination of broadband and short-period instruments) operated by GeoNet (the Geological hazard information for New Zealand; Petersen et al., 2011) and 12 temporary short-period stations installed and operated by Disaster Prevention Research Institute, Kyoto University, Japan (DPRI Okada et al., 2019), which operated at different time periods between 2009 and 2018. The earthquake catalog used for Chapters 3 and 4 are introduced in the respective Chapters. For the Whataroa study we used 159 out of the 160 three-component stations (one station was not functioning) which were spaced $\sim 35$ $\mathrm{m}$ apart and were active from $16^{\text {th }}$ to $21^{\text {st }}$ January 2016 (6 days) ( Townend et al., 2018). In conjunction with the dense arrays, we used data from five three-component Reftek 
short-period (2 Hz) stations deployed during the 2016 Whataroa Active Source Seismic Experiment (Lay et al., 2016, 2020; Townend et al., 2018), four shallow borehole $(\sim 27$ $\mathrm{m})$ short-period $(4.5 \mathrm{~Hz})$ stations deployed in and around the DFDP-2 (phase two of Deep Fault Drilling Project) drill-site during the drilling (Chamberlain et al., 2017b) and one SAMBA (Southern Alps Microearthquake Borehole Array) station (WHAT2 $2 \mathrm{~Hz},-81 \mathrm{~m}$; Boese, 2012). Lastly, for the numerical simulation study, we analyzed synthetic shear waveforms propagating in 1-D and 3-D anisotropic media for a suite of scenarios.

\subsection{Methods}

Each of the three main research Chapters (Chapter 3, 4 and 5) has it own method section where we describe the methods we used for analysis. Here we provide additional details for 2 key methods used in this thesis. We start with the method we used to estimate shear wave splitting parameters and give a brief description of the spatial averaging technique used for the $\phi$ measurements. We conclude with a description of the 3D simulation package used for waveform simulation in Chapter 5.

\subsubsection{Estimating shear wave splitting parameters}

One of the properties of S-waves in an isotropic medium is that they have linearly polarised particle motion, i.e. a particle on the raypath vibrates only in one direction. When an S-wave with an arbitrary polarization direction enters an anisotropic medium travelling in a direction other than the axis of symmetry, the wave splits into the two waves $S_{1}$ and $S_{2}$, with perpendicular polarizations, one travelling faster than the other. This leads to a time shift between the two wavelets (a split waveform) and causes the particle motion to change. When the time shift is small in comparison with the period of the wave, the particle motion changes from being linear to being elliptical. The only exceptions to this are S-waves that are entering the anisotropic medium with a polarization direction parallel to either the fast or the slow direction. These waves will not split since they only have a component in one of the two directions. 
The general idea used in estimating SWS parameters is to find the parameter combination (fast orientation direction and delay time) that will un-split an anisotropic S-wave from it's fast and slow waveform (Figure 2.1A), to match an elliptical particle motion that produces a linearly polarized S-wave (Figure 2.2B), so that the original isotropic waveform will be restored (Figure 2.1B). The process to un-split the S-wave involves the following steps, and assuming knowledge of the fast direction, slow direction (perpendicular to the fast direction), delay time and the initial polarization (Gerst and Savage, 2004; Wessel, 2010; Savage et al., 2010a). Firstly, the horizontal components are plotted in N-E coordinates (as a hodogram, display of the particle motion; Figure 2.2) to observe the complicated seismogram and elliptical particle motion. Secondly, the components and hodogram are rotated to follow the coordinate system of the fast and slow direction. If the fast direction has been correctly identified then in this coordinate system, the waveforms of the fast and the slow direction look similar (Figure 2.1B). The only obvious difference between them in the normalised plot is the delay time, $\delta t$ between the fast and slow wave (Figure 2.1A). Thirdly, the matched fast waveform is shifted back by $\delta t$ so the waveforms fit on top of each other. Linearity of the hodogram (Figure 2.2B) is then achieved and the angle of the hodogram with respect to north is controlled by the initial polarization. By doing so, all energy will be on the component of the initial polarization, and the other component will contain no energy.

In the case where we know the true splitting parameters $(\phi, \delta t)$ and the true initial polarization, un-splitting the shear wave turns out to be trivial. In the case where the splitting parameters are known but the the initial polarization is not known, the wave's horizontal components are rotated and time shifted by the splitting parameters $(\delta t$ and $\phi)$ making the wave seem as if it is propagating in an isotropic medium. In this case, a furthure technique has to be implemented to determine the initial polarization. The assumption that a wave in an isotropic medium with its component rotated to the initial polarization will have linear particle motion can be used to determine the initial polarization (Silver and Chan, 1991; Wessel, 2010). A common technique is to use the eigenvalues $\left(\lambda_{i}\right)$ of the covariance matrix of the horizontal components (Silver 

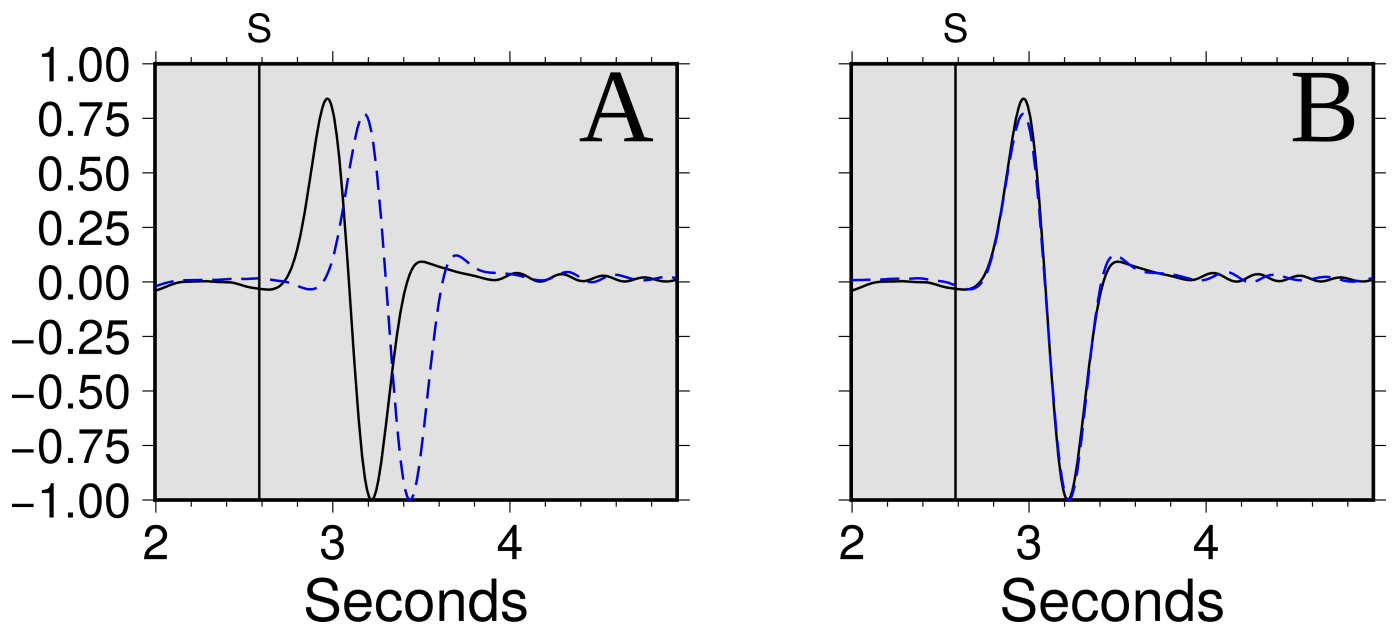

Figure 2.1: Example of split and unsplit waveform. The left panel shows the split shear waveforms (showing the fast with black solid waveform and slow with blue dashed waveform, both normalised to the maximum absolute value on the trace). The right panel shows the "corrected" (unsplit) waveform. The black vertical line is the S-phase arrival time.

and Chan, 1991; Wessel, 2010; Savage et al., 2010a; Walsh et al., 2013). These provide valuable information and less observer bias or subjectivity than a procedure where the observer picks the initial polarization by looking at diagnostic plots (e.g. Figure 2.3) and judging the direction of the particle motion (Silver and Chan, 1991; Savage et al., 2010a; Wessel, 2010; Walsh, 2012). If the initial polarization is known, such as in the case of an SKS wave (tele-seismic waves) where it is transverse, you can minimize the energy on the component perpendicular to the initial polarization (Silver and Chan, 1991).

In the case where both the splitting parameters $(\phi, \delta t)$ and the initial polarization are unknown, an arbitrary pair of splitting parameters are used to un-split the waveform and rotate it to the initial polarization. The best parameters will be the splitting parameters that truly un-split the waveform and the best initial polarization will be the wave's polarization-direction that removes ellipticity, producing a linear particle motion. To do this, we conduct a grid search over pairs of splitting parameters, and map the resulting smaller eigenvalue $\lambda_{2}$ in a 2D plot (Figure 2.5, $\lambda_{2}$ is described later in this Section). The assumption is made that the $\delta t, \phi$ pair that produces the smallest eigenvalue is the best pair, since it produces the most singular covariance matrix and therefore the most linear particle motion (Savage, 1999; Teanby et al., 2004a,b; Wessel, 

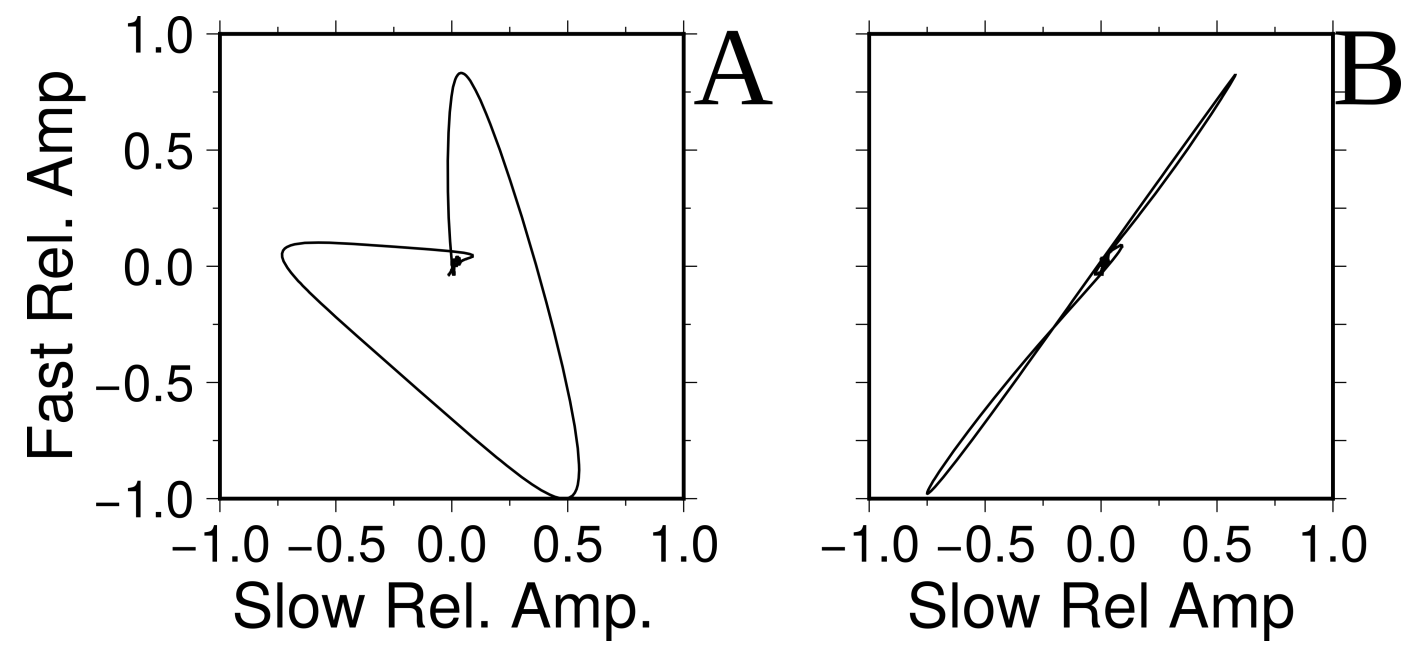

Figure 2.2: Example of horizontal particle motion of the waveform for split and unsplit waveform. The left panel show the splitted hodogram (horizontal particle motion of the waveform) showing an elliptical particle motion. The right panel shows the "corrected" hodogram, showing a linear particle motion.

2010; Savage et al., 2010a).

Automated methods can be used to analyze large data sets, although visual inspection is useful for checking the results. There are currently several automated methods used in shear wave splitting analysis; the cross-correlation method (Fukao, 1984), the covariance matrix method (Silver and Chan, 1991), the aspect ratio method (Shih et al., 1989) and most recent a combination of the Silver and Chan (1991) algorithm and the cluster analysis method described by Teanby et al. (2004a), which has been implemented by Wessel (2010) and Savage et al. (2010a).

The Silver and Chan (1991) algorithm is one of the most widely used methods for determining the splitting parameters. The Silver and Chan (1991) approach defines a splitting operator, $\Gamma$, that produces a split shear wave when applied to a shear wave, as the following:

$$
\Gamma \equiv e^{-i \omega \delta t / 2} \hat{\boldsymbol{f}} \hat{\boldsymbol{f}}^{T}+e^{-i \omega \delta t / 2} \hat{\boldsymbol{s}} \hat{\boldsymbol{s}}^{T}
$$

where $\hat{\boldsymbol{f}}$ and $\hat{\boldsymbol{s}}$ are the fast and slow S-wave vectors determined from the eigenvalue problem in equation 1.17. Here we follow both Silver and Chan (1991) and Walsh et al. (2013) in the describing the equations relating to the Silver and Chan (1991) method. $\delta t$ can be found using the associated eigenvalues of $\hat{\boldsymbol{f}}$ and $\hat{\boldsymbol{s}}$, and the squared 

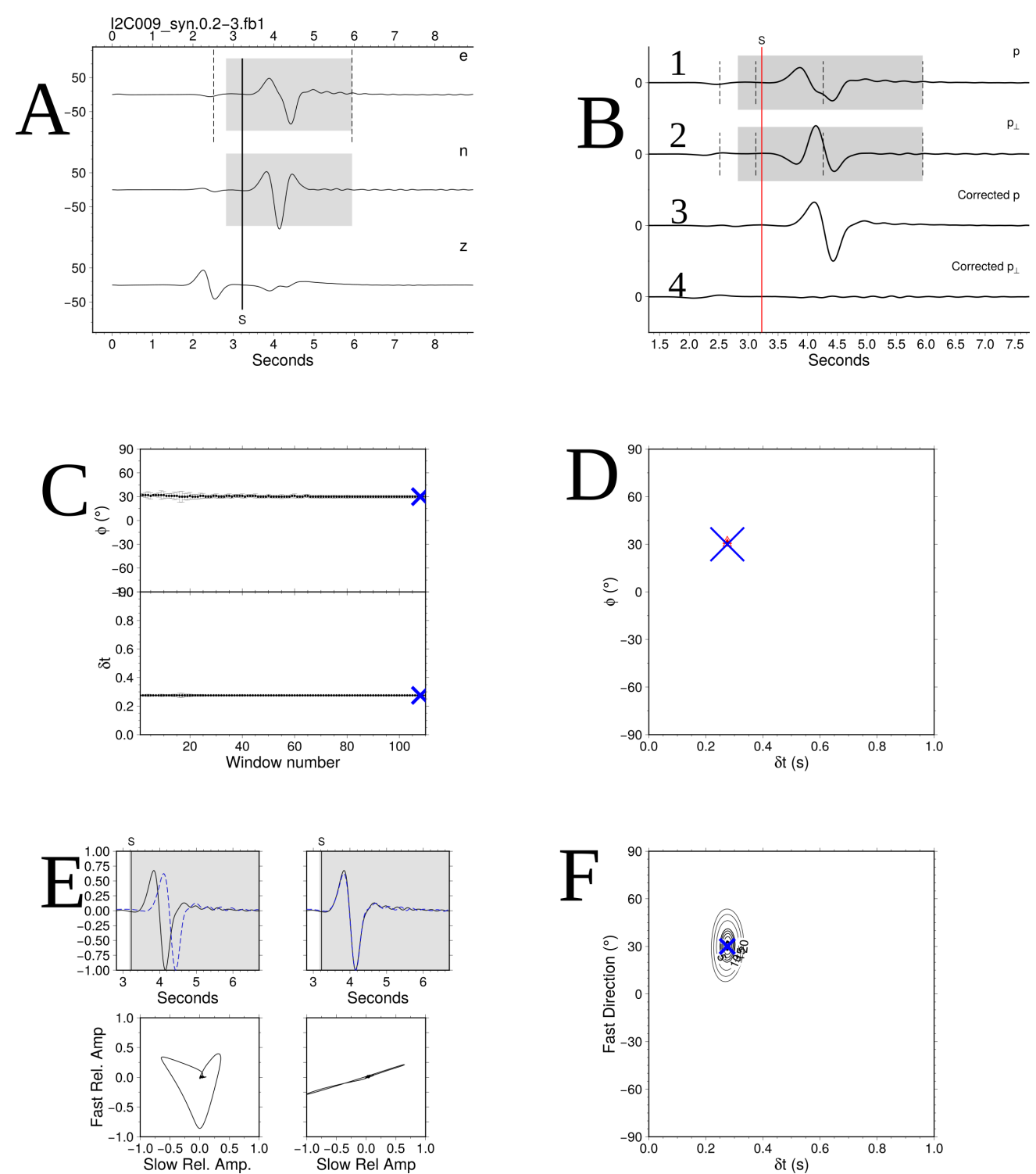

Figure 2.3: Example of MFAST diagnostic plots for a good quality measurement from synthetic waveform: (A) original dta (B) Filtered 3C seismogram (e,n,and z) waveforms. The solid vertical line is the local S-phase arrival and the gray box shows the dynamic window used for the analysis. 1. Original seismogram rotated into the initial polarization $(p), 2$. Original seismogram rotated perpendicular to the initial polarization $\left(p_{\perp}\right), 3$. Corrected seismogram rotated to the initial polarization, 4. Corrected seismogram rotated perpendicular to the initial polarization. (C) and (D) show the estimated $(\phi, \delta t)$ for each of the windows, and also indicate the optimal window selected as the best estimate. (E) The left panel shows the splitted (fast and slow) shear waveforms (top) and the corresponding hodogram (horizontal particle motion of the waveform) showing an elliptical particle motion (bottom). The right panel shows the "corrected" waveform (top) and it corresponding hodogram, showing a linear particle motion (bottom). (F) Shows the contour plot of $\lambda$ as a function of $\phi$ versus $\delta t$; The solution with the smallest second eigenvalue, $\lambda_{2}$ of the covariance matrix is marked by blue cross and the $95 \%$ confidence region of this solution is denoted by the contour outlines. 

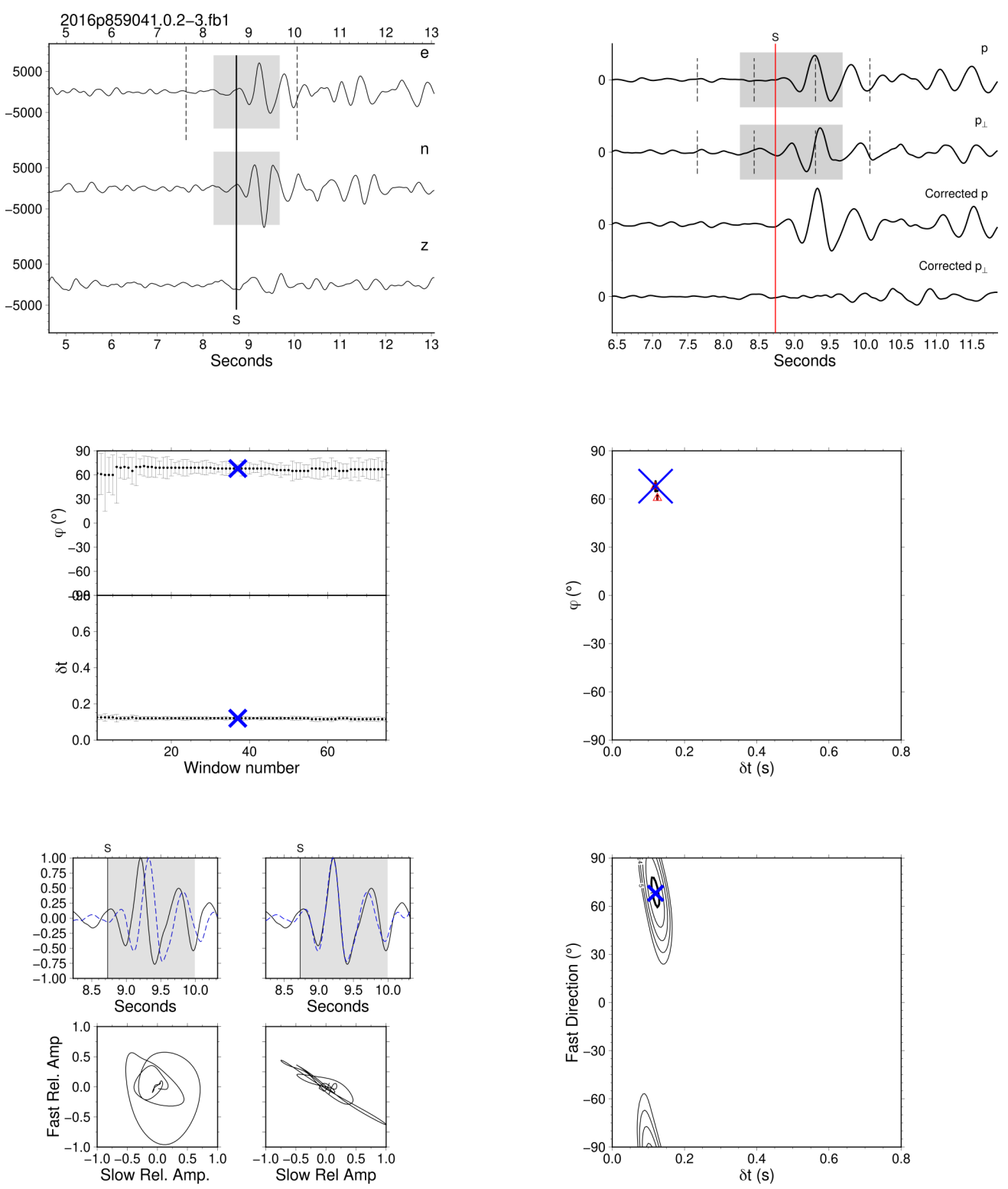

$$
\begin{aligned}
& \text { event 2016p859041.0.2-3.fb1 } \\
& \text { depth: } \quad 9.21875 \mathrm{~km} \\
& \text { distance: } \quad 33.2961 \mathrm{~km} \\
& \text { magnitude: } 3.8353 \\
& ------------------ \\
& \text { wbeg: }-1.10--0.30(5) \\
& \text { selected: } 8.2314-9.6787 \text {, length: } 1
\end{aligned}
$$

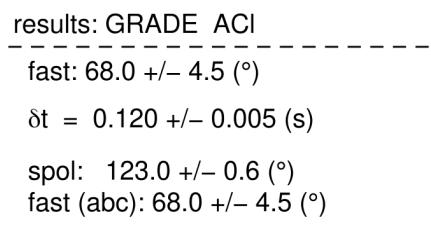

Nolumes/GeoPhysics_05/users-data

Figure 2.4: Example of MFAST diagnostic plots for a good quality measurement from real waveform: follows the same direction as Figure 2.3

shear velocities of the fast and slow wave, $V_{f s}^{2}$ and $V_{s s}^{2}$ respectively. Considering a small amount of anisotropy, $\delta t$ is expressed in terms of a relative perturbation in shear 
velocity, $\delta \hat{V}_{s}=V_{s 0}^{-1}\left(\delta V_{f s}-\delta V_{s s}\right)$, as:

$$
\delta t=V_{s 0}^{-1} L \delta \hat{V}_{s}
$$

where $V_{s 0}$ is the shear velocity in an isotropic medium and $L$ is the path length of the shear wave (Silver and Chan, 1991; Walsh et al., 2013). Consider the equation of a plane wave displacement as a function of frequency at an arbitrary position:

$$
u(\omega)=\boldsymbol{\omega}(\omega) e^{-i \omega t} \cdot \mathbf{n}
$$

where $\boldsymbol{\omega}(\omega)$ is a wavelet function and $\mathbf{n}$ is the ray propagation unit vector such that $A=\boldsymbol{\omega}(\omega) \mathbf{n}$. Applying the splitting operator $\Gamma$ to equation 2.3 gives the equation for a split waveform, $u_{s}$ :

$$
u_{s}(\omega)=\boldsymbol{\omega}(\omega) e^{-\omega t} \Gamma(\phi, \delta t) \cdot \mathbf{n}
$$

where $\phi$ is defined as the angle between $\hat{\boldsymbol{f}}$ and $\mathbf{n}$ (Walsh, 2012; Walsh et al., 2013). Defining the tensor:

$$
\delta T=\frac{\delta t}{2}\left[\hat{\boldsymbol{f}} \hat{\boldsymbol{f}}^{T}-\hat{\boldsymbol{s}} \hat{\boldsymbol{s}}^{T}\right]
$$

we can express $\Gamma$ as:

$$
\Gamma=e^{-i \omega \delta T(\phi, \delta t)}
$$

In estimating the values of $\phi$ and $\delta t$ for a given split waveform, $u_{s}$, the Silver and Chan (1991) approach searches for the inverse shear operator, $\Gamma^{-1}$ that will return the unsplit wave in equation 2.3. To find $\Gamma^{-1}$, a grid search over possible combinations of $\phi$ and $\delta t$ is undertaken in order to find the parameters that return the most linear, and therefore unsplit, particle motion. The linearity of a particle motion function is assessed by calculating the eigenvalues of its two dimensional time-domain covariance matrix. The covariance matrix between two orthogonal components of ground motion, 
for any pair of splitting parameters $\phi$ and $\delta t$, is defined as:

$$
C_{i j}(\phi, \delta t)=\int_{\infty}^{-\infty} u_{i}(t) u_{j}(t-\delta t) d t
$$

For a linear wave, $C$ will have only one nonzero eigenvalue, $\lambda_{1}=\int_{\infty}^{-\infty} \omega(t)^{2} d t$ with a corresponding eigenvector, $\mathbf{n}$. In the presence of anisotropy, $C$ will have two nonzero eigenvalues, $\lambda_{1}$ and $\lambda_{2}$. The process of finding the best $\Gamma^{-1}$ is to find the combination of $\phi$ and $\delta t$ that results in only one nonzero eigenvalue, $\lambda_{1}$, of the covariance matrix. However, in the presence of noise the second eigenvalue, $\lambda_{2}$ will not be exactly zero, hence the method finds a combination of $\phi$ and $\delta t$ that minimise $\lambda_{2}$. Walsh (2012) and Walsh et al. (2013) give a detail overview of the Silver and Chan (1991) method and the involved equations.

In this study, we are faced with a case where both the splitting parameters and the initial polarization are unknown. We estimate the SWS parameters using the Multiple Filter Automatic Splitting technique, MFAST, (Wessel, 2010; Savage et al., 2010a; Walsh et al., 2013) which uses a combination of the Silver and Chan (1991) algorithm and the cluster analysis method described by Teanby et al. (2004a). As stated earlier, the procedure is to find the inverse splitting operator that best removes the splitting. A series of band-pass filters are first applied to the waveforms. The product of the bandwidth and the signal-to-noise ratio after filtering are used to determine the best three filters. Splitting measurements using the Silver and Chan (1991) method, with the corrected version of the error calculation (Walsh et al., 2013), are determined on all three filtered records (Figure 2.3A shows the best filters). Rotation of $\phi$ values between $-90^{\circ}$ and $90^{\circ}$ with a $1^{\circ}$ increment and delay time correction of $\delta t$ values from 0 to 0.8 s for the Kaikôura study and 0 to 0.4 for the Whataroa study, with a 0.01 $\mathrm{s}$ increment, are applied to a selected time window (gray region in Figure 2.3E and F) of the east and north components of the filtered record. The $(\phi, \delta t)$ pair that best removes the splitting (Figure 2.3c), as measured by the smallest eigenvalue of the corrected covariance matrix, is the measurement for the given time window.

This procedure is then repeated for 75 windows covering slightly different times. 


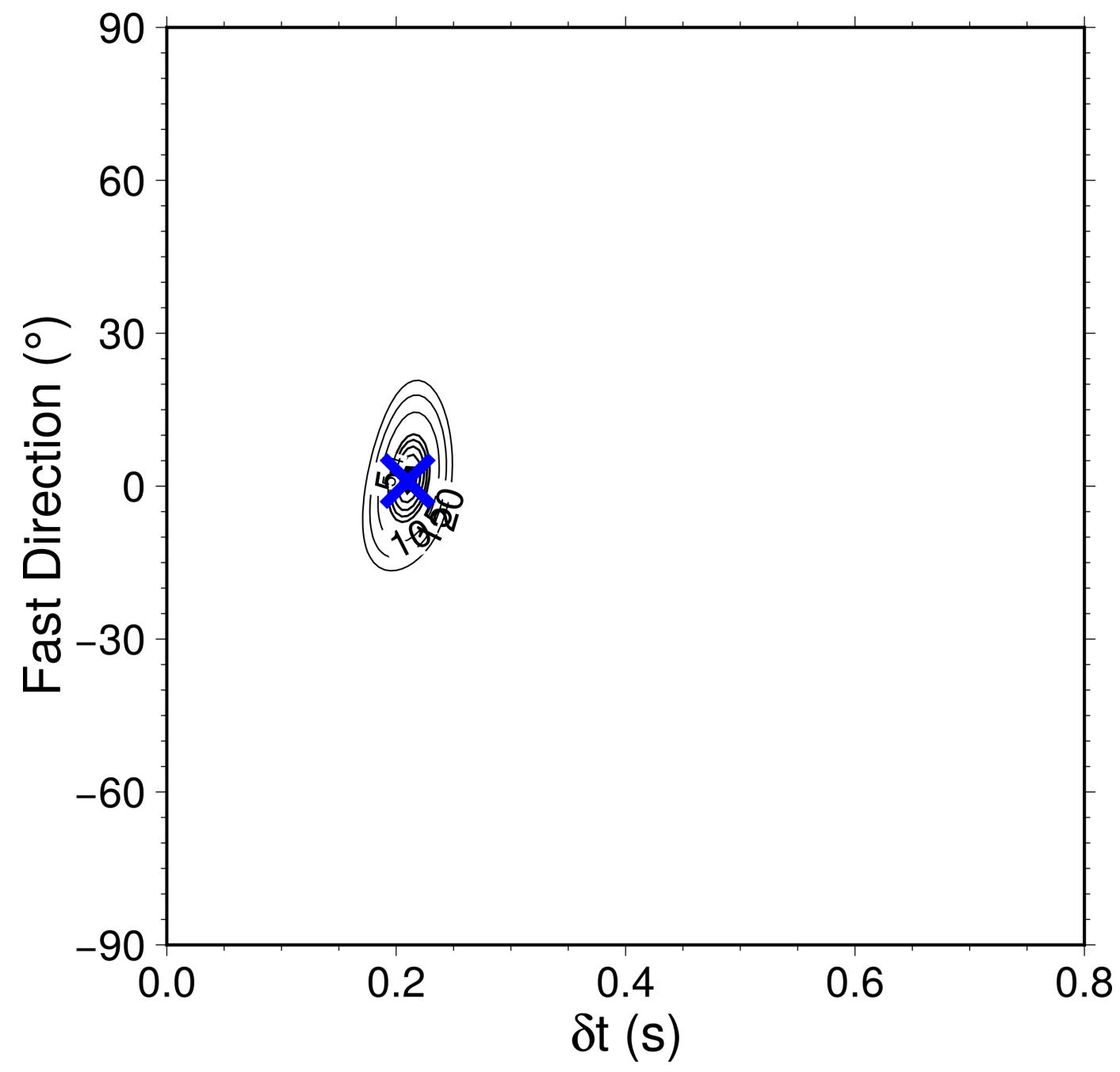

Figure 2.5: Contour plot of $\phi$ versus $\delta t$; The solution with the smallest second eigenvalue, $\lambda_{2}$ of the covariance matrix is marked by blue cross and the $95 \%$ confidence region of this solution is denoted by the contour outlines.

Measurements for a given time window are mostly dependent on the bandpass filter applied to the data. These 75 windows are selected based on the dominant period around the shear wave. MFAST automates the selection process and the optimal filter is determined based on the dominant period of the shear waveform, which is determined from the signal-to-noise ratio of the series of band-pass filters used. Cluster analysis over the 75 windows measurements is used to pick the final measurement for the considered filter and to calculate the associated uncertainty (Teanby et al., 2004a). The measurements are graded from A to D depending on the consistency between the $(\phi$, $\delta t)$ measurements from the different windows and their uncertainty. Events for which no measurable splitting occurs (or for which $\phi$ is within $20^{\circ}$ of parallel or perpendicular 
to incoming polarization $\left(\phi_{i n}\right)$ are given a null grade. MFAST also provides a measure of the incoming polarization by computing the eigenvalues of the corrected components after SWS is removed. The $\phi_{i n}$ corresponds to the polarization of the wave before it enters the last anisotropic layer responsible for the measured $\phi$ value. For a detailed description of the method see (Wessel, 2010; Savage et al., 2010a).

\section{Null measurements}

In determining reliable SWS results the diagnostic plots from MFAST (Figure 2.3 and 2.4 for synthetic and real waveforms respectively) can be used. A result is considered reliable if; (1) the fast and slow shear waves show similar waveforms and the particle motion after correcting for splitting is linearized (Figure 2.3E) (2) the energy on the corrected transverse component is minimized (Figure 2.3B) (3) the 2D error contour is well constrained and has a unique solution (Figure 2.3F).

SWS measurements are considered "Null" measurements if there is an absence of detectable splitting. SWS are likely not to occur if: (1) there is no anisotropy in the shear wave's propagating path or (2) if the initial polarization of the incoming shear wave aligns with either the fast or slow orientations (Wessel, 2010; Savage et al., 2010a; Balfour et al., 2012). In such cases, the estimated SWS parameters are not reliable. Currie et al. (2004) also suggests that when there is complex anisotropy, such as multiple or steeply dipping anisotropic layers, null measurements can also be observed. Often, when null measurements are recorded there are two distinct possible fast directions with no resolvable delay time (Wessel, 2010; Savage et al., 2010a; Balfour et al., 2012). An example is shown in Figure 2.6 and Figure 2.7 (synthetic and real waveforms respectively) where the contour plot of Figure 2.6F shows the two possible fast directions. In this thesis, null measurements are removed unless otherwise stated. We consider any measurements of fast polarization directions that are within $20^{\circ}$ of the incoming polarization of the wave as null results. 

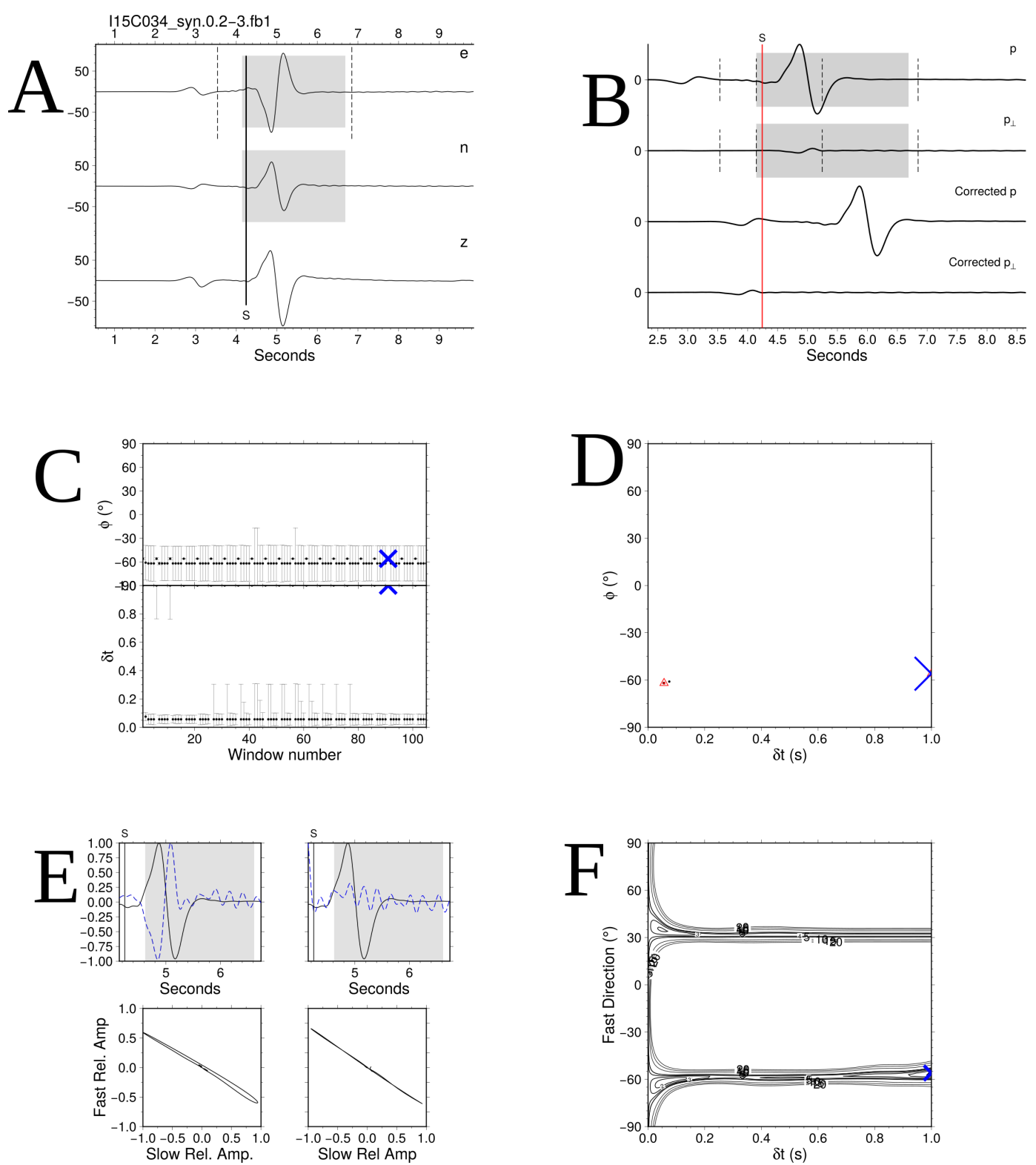

Figure 2.6: Example of a null measurement from MFAST diagnostic plots (synthetic waveform), following the same description as in Figure 2.3. (F) The contour plot of $\phi$ versus $\delta t$. The two possible fast directions observed on the contour plot is an indication of a null measurement. $\delta t$ is not constrained because there is no anisotropy.

\subsubsection{Spatial averaging of fast direction measurements}

To examine spatial variations of $\phi$ associated with local splitting parameters, we utilized a spatial averaging technique implemented in a well established TESSA (Tomography Estimate and Shear wave splitting Spatial Average) package which was developed by Johnson et al. (2011). TESSA (a pseudo 2D tomography) is based on a simplified and linear relationship between SWS parameters and path length (straight-line ray tracing 

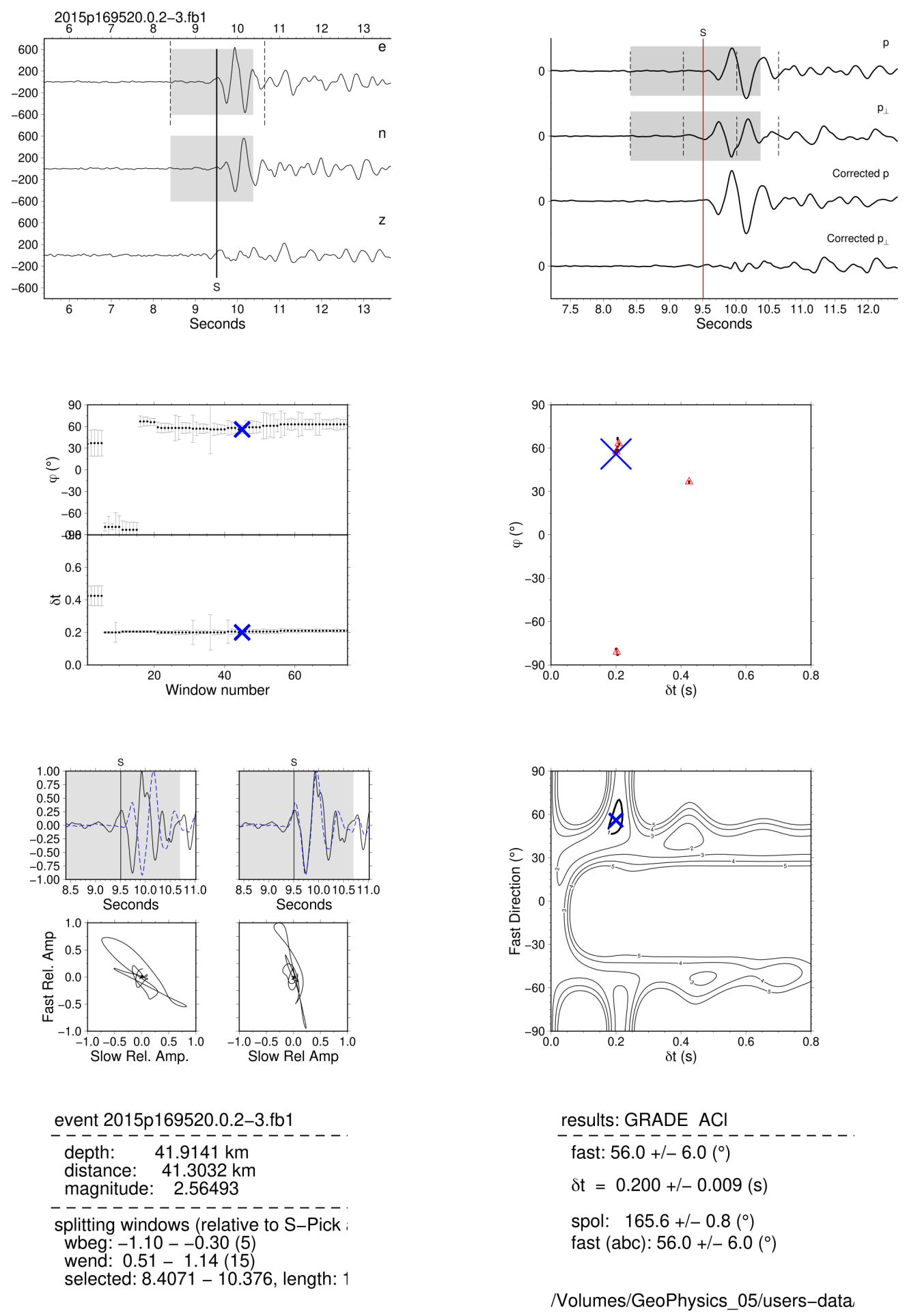

Figure 2.7: Example of MFAST diagnostic plots for a null measurement from real waveform: follows the same direction as Figure 2.6 and 2.3 
between station and event; Johnson et al., 2011). The spatial averaging technique uses the same grid as the $\delta t$ tomography method in TESSA. The gridding is done using the quadtree grid algorithm (Townend and Zoback, 2001). The algorithm iteratively divides the study region into blocks. The blocks are iteratively divided into smaller blocks when the number of raypaths exceed the maximum number allowed (in our case 80) until no blocks exceed the maximum raypaths or the grid spaces reach the minimum size (in our case $5 \times 5 \mathrm{~km}$ ).

For each $\phi$ node (raypath) within a given quadtree grid block we provide constraints on $\phi$ by assigning a weight (Johnson et al., 2011). Because we observed that the splitting occurred later in the ray-path (upper few kilometers; see Section 3.5.3) we used a distance (d) based weighting of $1 / d^{2}$. This heavily weights the measurements closer to the station. The spatial averages of $\phi$ measurements in each block are computed using a circular statistics approach (Berens, 2009) and are estimated only when the standard deviation of fast orientations in each grid is less than $30^{\circ}$ and the standard error of the mean is less than $10^{\circ}$. If there was fewer than $20 \phi$ measurements in a block, we discard that block. This is done to ensure that each block contains enough data to give a reliable average measurement. 


\subsubsection{SpecFem3D: Spectral finite element method}

Wave propagation in a layer-cake velocity model can be described based upon an analytical technique such as the reflectivity method (e.g. Booth and Crampin, 1985) or equivalent media theory (e.g. Schoenberg and Muir, 1989). But these analytical techniques are often insufficient to explain the full range of phenomena that arise in complex heterogeneous Earth models such as spatial heterogeneity in velocity model, attenuation, scattering, free-surface topography and anisotropy. To account for a complex and realistic Earth model, one needs to turn to numerical methods. Numerical differential equation methods transform an original differential problem into a system of algebraic equations. In solving the wave equation, the governing equation can be treated in two forms; namely the "strong" and "weak" form. Strong methods are based on the equations of motion in their differential form, subject to certain boundary conditions (Robertsson et al., 2012) while the weak form uses the integral form of the wave equations.

Perhaps the most common means of solving the strong form of the wave equation is the finite-difference method (FDM) (e.g. Igel et al., 1995; Komatitsch et al., 2013). The FDM is often used because one can discretize the wave equation on a structured grid making it computationally efficient, applicable to simple models. It is also relatively accurate, and easy to implement parallel processing (Igel, 2016). However, accurately modelling complex boundaries and interfaces on a structured grid can be cumbersome (Igel et al., 1995; Igel, 2016). Moreover, the FDM is less accurate when implementing surface topography (free surface boundary conditions) due to the approximation of boundary conditions (Komatitsch and Tromp, 1999; Tromp et al., 2008; Robertsson et al., 2012). Another limitation with the FDM is the implementation of anisotropy in the models. Igel et al. (1995) describes the challenges of modeling wave propagation in an anisotropic medium using the FDM, especially on the implementation of the strain tensor. Although other studies (e.g. Lisitsa and Vishnevskiy, 2010; Bernth and Chapman, 2011; Saenger and Bohlen, 2012) show how to get around the challenges described by Igel et al. (1995), there are still limitations when including full anisotropic 
media in models (Igel, 2016). However, the use of the "weak form" equation makes the implementation of full anisotropic media trivial (Chevrot et al., 2004; Chen and Tromp, 2007). Fichtner (2010) and Moczo et al. (2007) present a comprehensive review of different finite-difference methods.

The "weak form" employs an integral form of the wave equation that contains the boundary conditions implicitly and provides a means to control the accuracy of the solution (Komatitsch et al., 2000). A successful weak form method for solving the seismic wave equation is the spectral-element method, SEM. The SEM is a generalized form of the finite-element method (FEM), based on the use of high-order piece-wise polynomial functions. The SEM also uses nodal quadrature, namely the Gauss-Lobatto-Legendre (GLL), quadrature for numerical integration over an element (e.g. Komatitsch et al., 2000). The use of GLL quadrature enables an efficient time-marching scheme that lends itself very well to calculations on parallel computers (Durufle et al., 2009). This makes the SEM more computationally efficient than classical FEM (e.g. Bao et al., 1998; Komatitsch and Tromp, 1999). Spatial accuracy can be increased by using higherorder polynomial functions and a refined mesh (Komatitsch and Tromp, 1999; Tromp et al., 2008). The SEM naturally accommodates boundary conditions in the presence of steep free-surface topography or major domain interfaces by allowing finite elements to follow local boundary waviness, in particular along the Earths surface (Priolo et al., 1994; Komatitsch and Tromp, 1999; Tromp et al., 2008). Another important feature the SEM has over other techniques is that the anisotropy strain components can easily be defined on the mesh element (Chen and Tromp, 2007). The SEM was originally developed to solve fluid dynamic problems (Canuto et al., 1988). Since then, it has been widely used to simulate seismic wave propagation in various scales and media (e.g. Faccioli et al., 1997; Tromp et al., 2008; Komatitsch et al., 2000; Peter et al., 2011; Chen and Tromp, 2007; Lee et al., 2009a,b)

For the three-dimensional (3-D) simulation in Chapter 5, we use the Spectral Finite Element Method, SpecFEM, which is a SEM designed to solve the full wave equation in a mesh of with hexahedral elements (Komatitsch and Tromp, 1999; Komatitsch et al., 2000; Qinya et al., 2004; Tromp et al., 2008). The technique takes advantage of the 
flexibility of a FEM with the accuracy of a spectral method, which has the potential to easily incorporate free-surface topography (Komatitsch and Tromp, 1999). Models built in SpecFEM3D can be fully heterogeneous or composed of any number of layers (Komatitsch and Tromp, 1999). This technique offers the flexibility needed to test the effects that scatterers, topography and focal mechanisms have on SWS measurements. SPECFEM3D is developed by Computational Infrastructure of Geodynamics (www.geodynamics.org/cig/software/specfem3d) and comes in two main versions: the local/regional version called the SPECFEM3D Cartesian, and the global scale version called SPECFEM3D Globe. Because our study is focused on a 3D local scale, we used SPECFEM3D Cartesian. For this thesis, any time we mention SPECFEM3D we refer to the SPECFEM3D Cartesian.

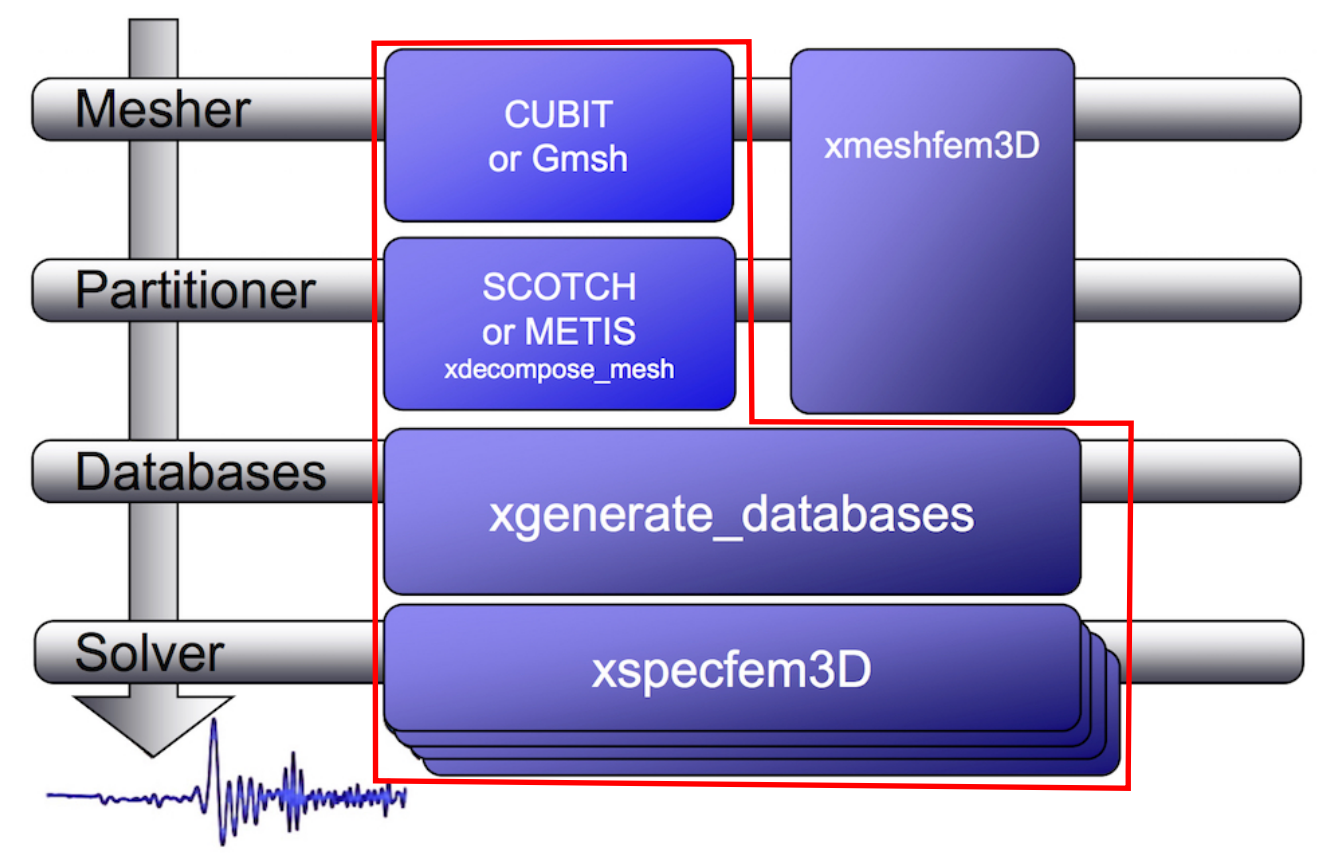

Figure 2.8: Illustration of the Schematic workflow used for SPECFEM3D simulation. The red region marks the workflow we used for our simulations. Figure from Pavlov (2012).

The schematic workflow for a SPECFEM3D simulation is shown in Figure 2.8. The work flow can be slightly different depending on the use of an internal or external meshing program. For our workflow we use an external meshing program, Cubit v 16.4 
(www.csimsoft.com/trelis.jsp) in conjunction with GeoCubit (a Python based script; Casarotti et al., 2008b). The SPECFEM3D program has an internal meshing tool "xmeshfem3D", but the tool lacks the flexibility to generate the meshes needed for our simulation.

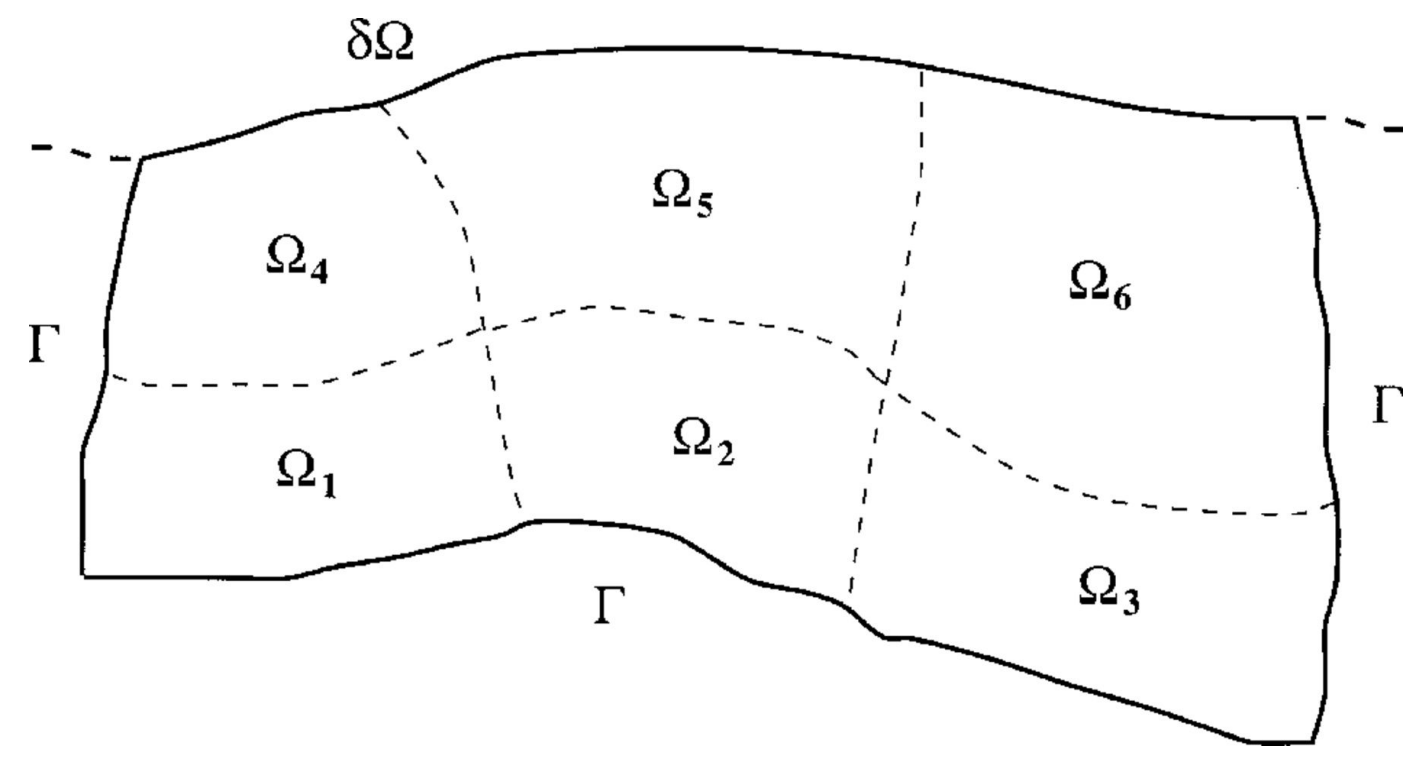

Figure 2.9: We subdivide the model $\Omega$, for the purpose of computation, into deformed cube elements (hexahedral element, see Figure 2.10) whose shapes are adapted to the edges of the model $(\delta \Omega$ and $\Gamma$ ). Figure from Komatitsch and Tromp (2002).

For the meshing, we subdivide the model volume, $\Omega$ into a number of non-overlapping hexahedral (deformed cube) elements $\Omega_{e}$, where $e=1,2, \ldots, n_{e}$ (Figure 2.9). The subdividing is done such that $\Omega=\cup_{e=1}^{n_{e}} \Omega_{e}$ (Komatitsch and Tromp, 1999; Komatitsch et al., 2000; Tromp et al., 2008). The mapping between Cartesian points $X=(x, y, z)$ within the hexahedral element, $\Omega_{e}$ and the model volume, $\Omega$ (Figure 2.10) within which the points can be denoted by local coordinate $\xi=(\xi, \eta, \zeta)$ where $-1 \leq \xi, \eta, \zeta \leq 1)$, can be expressed in equation 2.8 .

$$
X^{e}(\xi, \eta, \zeta)=\sum_{a=1}^{M} X_{a}^{e} N_{a}(\xi, \eta, \zeta)
$$

Where, $X^{e}$ are the $M$ nodes of the $\mathrm{e}^{t h}$ hexahedral element, $X_{a}^{e}=X^{e}\left(\xi_{a}, \eta_{a}, \zeta_{a}\right)$ and the shape function $N_{a}(\xi, \eta, \zeta)$, define the geometry of the element (Komatitsch and Tromp, 1999; Komatitsch et al., 2000; Tromp et al., 2008). The shape function, $N_{a}(\xi, \eta, \zeta)$ is the triple product of first and second degree Lagrange polynomials and their derivatives in 


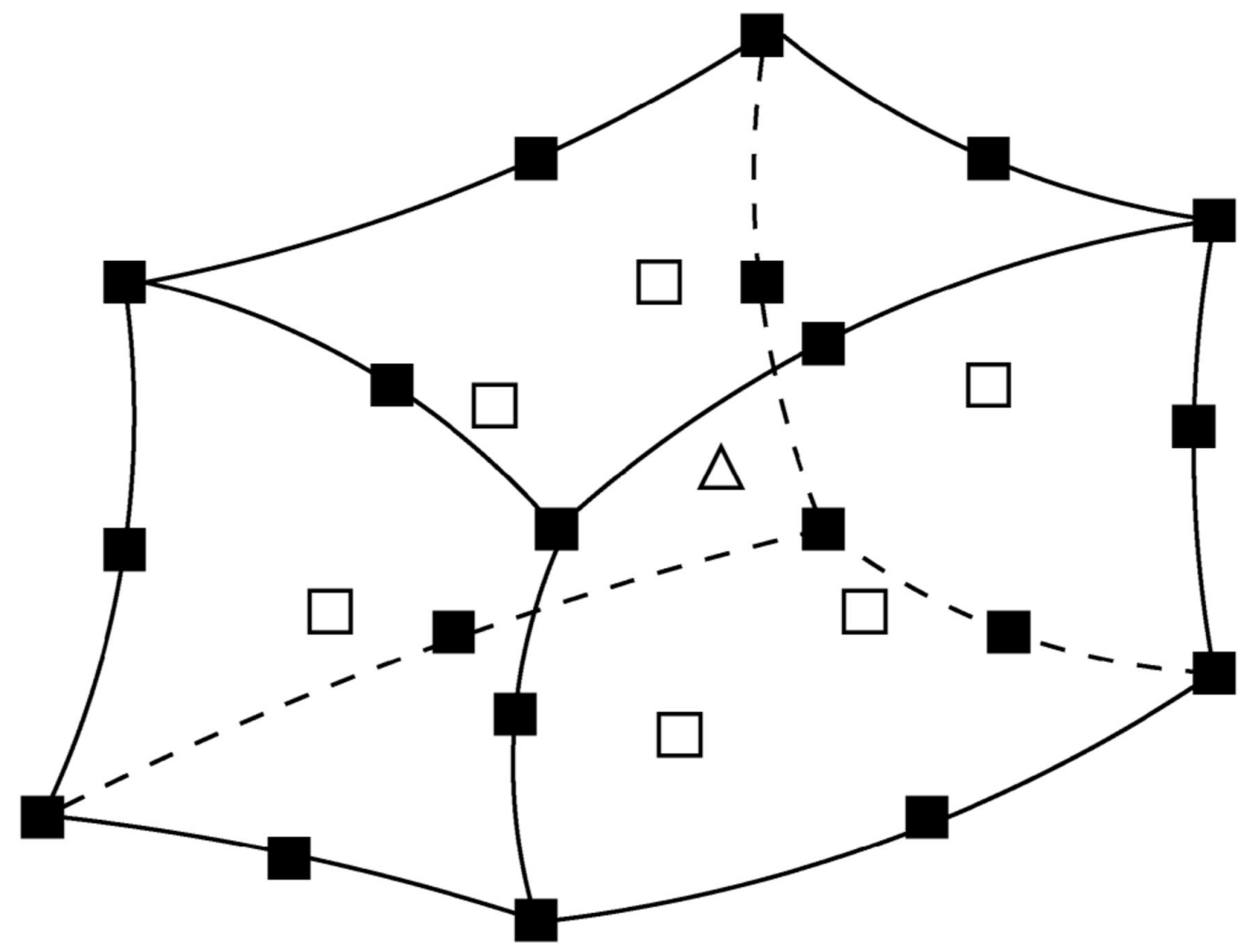

Figure 2.10: The geometry of a hexahedral finite element (deformed cube) can be defined in terms of the eight corners plus the 12 edges (totaling the 20 black squares) plus the 6 face centers (the 6 open squares in the middle of the sides) plus the center (the open triangle). This results in the 27 control nodes. The nodes are used to define the geometry of the elements and not for the interpolation and integration of functions. The interpolation and integration of functions are represented in terms of high-degree Lagrange polynomials on Gauss-Lobatto-Legendre interpolation points (GLL points). The figure and description are adapted from Komatitsch and Tromp (2002).

the three orthogonal directions of space in the reference cube (Komatitsch and Tromp, 1999, 2002; Tromp et al., 2008). The $N+1$ Lagrange polynomials of degree $N$ are defined as $N+1$ control points $-1 \leq \xi_{\alpha} \leq 1, \alpha=0 \ldots, n$ as (Komatitsch and Tromp, 1999; Tromp et al., 2008):

$$
\mathcal{L}_{\alpha}^{N}=\frac{\left(\xi-\xi_{0}\right) \ldots\left(\xi-\xi_{\alpha-1}\right)\left(\xi-\xi_{\alpha+1}\right) \ldots\left(\xi-\xi_{N}\right)}{\left(\xi_{\alpha}-\xi_{0}\right) \ldots\left(\xi_{\alpha}-\xi_{\alpha-1}\right)\left(\xi_{\alpha}-\xi_{\alpha+1}\right) \ldots\left(\xi_{\alpha}-\xi_{N}\right)}
$$

where $\mathcal{L}$ is a Lagrange polynomial. When the degree of Lagrange polynomials have $N=1$, then number of control points will be $\xi=-1$ and $\xi=1$ resulting in two polynomials (Komatitsch and Tromp, 1999). Similarly, if $N=2$, then the control points will be $\xi=-1, \xi=0$ and $\xi=1$, which then leads to three polynomials. Thus 
the control points $\xi_{\alpha}$ are chosen to be $N+1$. The control points are also refered to as the Gauss-Lobatto-Legendre (GLL) points (Komatitsch and Tromp, 1999; Komatitsch et al., 2000). These control points are used to discretize the wave field in each element. Illustration of the Lagrange polynomials and GLL points is shown in Figure 2.11. The higher the number of Lagrange interpolants, $N$, (higher GLL points) the higher the accuracy. This accuracy comes with an expensive computational cost (Chaljub et al., 2007). Often a balance is struck between the accuracy and computational cost. For our study we used a Lagrange interpolants of degree $N=4$ which gives us $125,(N+1)^{3}$, GLL points and this specification has been suggested by several studies (Komatitsch and Tromp, 1999; Chaljub et al., 2007; Tromp et al., 2008).
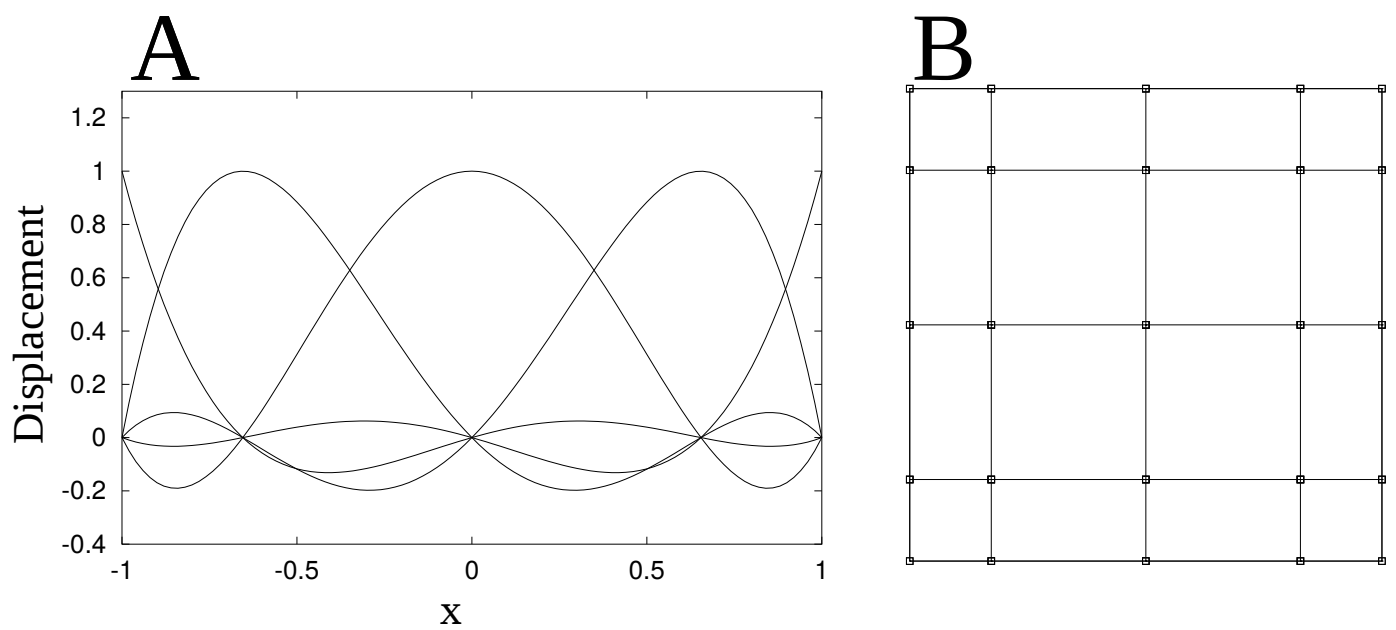

Figure 2.11: A. Lagrange interpolants of degree $N=4$ on the reference interval $\xi=[-1$, 1]. The corresponding $N+1$ (5) Gauss-Lobatto-Legendre points can be distinguished along the horizontal axis of A. All Lagrange polynomials are, by definition, equal to 1 or 0 at each of these points (Komatitsch and Tromp, 1999; Chaljub et al., 2007). B. When a polynomial of degree $N$ is used to discretize the wave field, each spectral element will contain $(N+1)^{3}$ grid points (Gauss-Lobatto-Legendre points) for the 3D case. For representation, we show the $2 \mathrm{D}$ face of the $3 \mathrm{D}$ element, which contains $(N+1)^{2}=25$ grids point (Chaljub et al., 2007; Komatitsch and Tromp, 2002). Figure from (Chaljub et al., 2007).

After the mesh generation, we use the "xdecompose_mesh_SCOTCH" tool to partition the mesh into splices based on the number of CPUs. This process helps with parallel simulation/computation of the wave equation. We then generate the necessary database (which includes the elastic properties, anisotropy parameters and topography) needed by the solver with the "xdecompose_mesh". After the database generation, we 
run the "xspecfem3D" tool to simulate the waveforms. The "xspecfem3D" tool is controlled by a parameter file where most of the parameters such as type of simulation (forward simulations or adjoint simulations), the number of time steps of the simulation, length of each time step in seconds and absorbing boundary conditions are specified. 


\title{
3 Spatial and Temporal Analysis of Seis-
}

\author{
mic Anisotropy Associated with the Cook \\ Strait and Kaikōura Earthquake Sequence
} in New Zealand

\section{Abstract}

Large earthquakes can diminish and redistribute stress, which can change the stress field in the Earth's crust. Seismic anisotropy, measured through shear-wave splitting (SWS), is often considered to be an indicator of stress in the crust because the closure of cracks due to differential stress leads to waves polarized parallel to the cracks traveling faster than in the orthogonal direction. We examine spatial and temporal variations in SWS measurements and the $V_{p} / V_{s}$ ratio associated with the 2013 Cook Strait (Seddon, Grassmere) and 2016 Kaikōura earthquakes in New Zealand. These earthquake sequences provide a unique dataset, where clusters of closely-spaced earthquakes occurred. We use an automatic, objective splitting analysis algorithm and automatic local S-phase pickers to expedite the processing and to minimize observer bias. We present SWS and $V_{p} / V_{s}$ measurements for over 40,000 crustal earthquakes across 36 stations spanning close to $5 \frac{1}{2}$ years between 2013 and 2018. We obtain a total of 102,260 (out of 398,169) high-quality measurements. We observe significant spatial variations in the fast polarization orientation, $\phi$. The orientation of gravitational stresses is consistent with most of the observed anisotropy. However, multiple mechanisms (such as structural, tectonic stresses and gravitational stresses) may control some of the observed crustal anisotropy in the study area. Systematic analysis 
of SWS parameters and $V_{p} / V_{s}$ ratio revealed that apparent temporal variations are caused by variation in earthquake path through spatially varying media.

\subsection{Introduction}

Stress in the Earth is an essential factor in earthquake genesis. Earthquakes are caused by the sudden rupture of rocks along faults exposed to differential stress in the Earth's crust. Earthquakes occur when the accumulated shear stress exceeds the strength of faults or fractures in the rock. Thus, crustal stresses are the immediate driving forces of earthquakes (Zoback and Zoback, 2002). A variety of techniques have been devised to measure crustal stresses. The most direct technique to determine crustal stresses is through strain measurements in boreholes, where both stress magnitudes and orientations can be determined. However, drilling boreholes to seismogenic depths is a difficult and expensive enterprise (Townend and Zoback, 2000; Zoback and Zoback, 2002). Another well-established method is the inversion of the earthquake focal mechanisms to determine the stress orientations in the region where the earthquake occurred (Michael, 1984; Hardebeck and Michael, 2006; Arnold and Townend, 2007). Geodetic techniques such as using GNSS (Global Navigation Satellite System) and InSAR (Interferometric Synthetic Aperture Radar) measurements, which can be used to infer strain rates, which can also be interpreted as changes in stress (Hardebeck and Michael, 2006; Hardebeck and Okada, 2018). In most regions, shear wave splitting (SWS) parameters can also serve to estimate the orientation of the principal horizontal stresses. Thus, stress orientations can be inferred from measuring crustal seismic anisotropy through SWS (Crampin, 1984; Savage et al., 2016; Cochran and Kroll, 2015) and changes in SWS parameters can also be interpreted as changes in the state of stress.

Seismic anisotropy is the dependence of seismic velocity on direction, as caused by the elastic properties of rocks. The estimation of the geometry and strength of seismic anisotropy is often measured through shear wave splitting. SWS is a phenomenon that is observed when shear waves propagating through an anisotropic medium split into two nearly perpendicular phases that travel with different velocities. In the 
crust, anisotropy is often considered to be controlled by either stress (stress-induced anisotropy) or geological structures (structurally induced anisotropy) (Crampin, 1984; Babuska and Cara, 1991; Zinke, 2000; Boness and Zoback, 2006; Crampin et al., 2015; Savage et al., 2016). In the case of stress-induced anisotropy, we assume that cracks or micro cracks are randomly distributed. Differential stress will close cracks perpendicular to the maximum horizontal stress $\left(S_{H \max }\right)$, leaving open cracks only parallel to $S_{H \max }$. In the case of structurally induced anisotropy, the fast wave is oriented parallel to structural fabric or the orientation of the residual features of paleostress (Savage, 1999; Cochran et al., 2006).

SWS can be characterized by two parameters, the fast orientation $(\phi)$ and the delay time $(\delta t) . \phi$ is the bearing of the polarization of the faster wave (also referred to as the fast orientations) and $\delta t$ is the time lag between the two split waveforms. $\delta t$ depends on both the degree of anisotropy and the path length through the anisotropic medium. Although early studies concluded that stress-induced crack alignment is the principal cause of crustal anisotropy (Crampin, 1984; Babuska and Cara, 1991), several recent SWS studies, close to and away from the vicinity of major faults, have concluded that other possible causes of crustal anisotropy exist (Zinke, 2000; Matcham et al., 2000; Balfour et al., 2005; Boness and Zoback, 2006). A consistent pattern where $\phi$ measurements in close proximity to faults show fault-parallel orientations and $\phi$ measurements further away from faults are parallel to the principal horizontal stress (orientations) has been reported around the San Andreas Fault of California, (Zinke, 2000; Boness and Zoback, 2006), Wellington region and Marlborough Fault System (MFS) of New Zealand (Gledhill, 1991; Audoine et al., 2000; Matcham et al., 2000; Balfour et al., 2005; Karalliyadda and Savage, 2013).

Several researchers have used SWS to measure spatial and temporal variations in seismic anisotropy. Miller and Savage (2001) observed changes in fast polarization orientations before and after an eruption at Mt. Ruapehu volcano, New Zealand. They interpreted this as a variation in principal stress orientation, which they related to an increase in stress-related to magmatic intrusion or inflation. Roman et al. (2011) observed rotations of fast orientations that correlated with rotating fault plane solutions 
for earthquakes associated with volcanic activity at Soufrière Hills volcano in Montserrat. Bianco et al. (2006) observed variations in fast orientation and delay times before the 2001 eruption on Mt. Etna. They observed delay times exhibiting a sudden decrease shortly before the start of the eruption and variations in the fast orientation five days before the onset of the eruption. Zheng et al. (2008) also studied the temporal variations of shear-save splitting in the aftershock region of the 1999 Chichi earthquake, Taiwan. They also observed a decrease in delay times shortly before the Chichi mainshock and two Chiayi earthquakes.

The relations between the ratio of body-wave (P-wave and $\mathrm{S}$-wave) velocities, $V_{p} / V_{s}$, fluid content and pore pressure are well-established (e.g. Wadati and Oki, 1933; Nur, 1971; Ito et al., 1979; Dvorkin et al., 1999, and references therein). The $V_{p} / V_{s}$ ratio is one of the best parameters to indirectly identify fluid and crack migration within the crust, since pore fluid properties produce variations in seismic velocities (Dvorkin et al., 1999). In fluid-saturated rocks, high $V_{p} / V_{s}$ anomalies may indicate high pore pressure (e.g. Dvorkin et al., 1999; Nur, 1971). Variations in the $V_{p} / V_{s}$ ratio have been interpreted qualitatively by several studies to be related to the movement of fluids in the crust. Li and Vidale (2001) observed an increase in shear velocity of the fault zone rock around Johnson Valley, after the $1992 M_{w} 7.5$ Landers, California, earthquake (from 1994 to 1998). They interpreted the trend as an indication of fault healing by strengthening after the mainshock, which is most likely due to the closure of cracks that opened during the 1992 earthquake. They also observed that the fault-zone strength recovery was consistent with a decrease in the apparent crack density within the fault zone. Moreover, they observed a decrease in the $V_{p} / V_{s}$ ratio, which they explained as cracks near the fault zone being partially fluid-filled and becoming more fluid saturated with time (Li and Vidale, 2001). Chiarabba et al. (2009), using S- and P-wave arrival times, observed an increase in $V_{p} / V_{s}$ before the $M_{w} 5.31997$ Umbria-Marche earthquake in central Italy and attributed the trend to a pore pressure increase in fluid-filled cracks in the volume around the fault. The combination of variations in seismic anisotropy, $V_{p} / V_{s}$ ratio, and geodetic data have been reported by recent studies in volcanic areas and slow-slip regions (Savage et al., 2010b; Unglert et al., 2011; Zal et al., 2020). 
Around the Wellington region of New Zealand, Gledhill (1991) and Matcham et al. (2000) used local earthquakes to study the anisotropic structure. Audoine et al. (2000) also characterized the crustal anisotropy around the transition between the subduction and the oblique transform faulting boundaries. In the central MFS, they observed that fast polarization orientations were subparallel to the major faults and geological features and attributed this observation to the presence of metamorphosed schist beneath the MFS. At the eastern edges of the MFS, they observed that fast orientations were parallel to the orientation of the maximum horizontal compressive stress, $S_{H \max }$ which is consistent with crack induced anisotropy in the crust. In the North Island, their fast polarization orientations were parallel to the strike of the Hikurangi subduction zone as well as to the major geological features (Audoine et al., 2000). Balfour et al. (2005) described the upper plate stress regime above the southern end of the Hikurangi subduction zone, where the dextral MFS accommodates more than $80 \%$ of the relative plate motion (Townend et al., 2012). They examined the relationship between the $S_{H \max }$ orientations and the average fast orientations, and concluded that both stress and structural anisotropy exist around the MFS, but the dominant structural fabric of the MFS controls the seismic anisotropy measurement rather than $S_{H \max }$ (Balfour et al., 2005). Regional stress studies by Townend et al. (2012) and Balfour et al. (2005) around the study area revealed a uniform $S_{H \max }$ of $115^{\circ} \pm 16^{\circ}$ in the upper-plate extending across the northern South Island. Recently, Evanzia et al. (2017) used SWS analysis to distinguish between stresses around southern Hikurangi Margin, New Zealand, using SWS and focal mechanism measurements. They suggested that stresses in the overriding plate are likely related to bending stresses, gravitational stresses and tectonic loading.

In this study, we perform a systematic analysis of SWS parameters to investigate the physical mechanisms causing crustal anisotropy around the Marlborough and Wellington region, and to explore possible temporal variations in SWS measurements. The 2013 Cook Strait and 2016 Kaikōura earthquake sequences provide a rare dataset, where clusters of closely-spaced earthquakes with full azimuthal coverage occurred. We use this dataset to examine the hypotheses of structure and stress-induced anisotropy 
that are often used to explain anisotropy in the crust. The stress-induced hypothesis suggests that cracks aligned with the maximum horizontal compressive stress direction, $S_{H \max }$, remain open with a preferred orientation (Crampin and Booth, 1989; Savage, 1999) and thus induce anisotropy. The stresses acting on crustal tectonic settings can be split into two categories; (i) topographic stresses acting on the shallow crust (often referred to as gravitational stresses) and (ii) stresses driven by the relative plate motions or geodynamic processes (referred as tectonic stresses). Often, crustal anisotropy studies use $S_{H \max }$ from focal mechanism inversions to explain the stress hypothesis with little focus on $S_{H \max }$ from gravitational stresses. Araragi et al. (2015) and Illsley-Kemp et al. (2017) are two of the few studies that suggested gravitational stress as a possible explanation for crustal seismic anisotropy around Mt. Fuji (Japan) and the Northern Afar region (East Africa) respectively. In theory, the orientation of gravitationally induced horizontal stresses, $S_{H_{\max }}^{G r a v}$ have orientations at the peak of the mountains that are different from those on the slope. At the peak, $S_{H_{\max }}^{G r a v}$ is parallel to the strike of the mountain ridge, whereas, at the slope, it is perpendicular (Flesch et al., 2001; Hirschberg et al., 2018). In basins between two mountains ridges, the expected $S_{H_{\max }}^{G r a v}$ is often perpendicular to the strike of the ridges due to the compressional stresses on the slopes on either side of the basin (Hirschberg et al., 2018). Here, we examine how well both stresses explain the crustal anisotropy in the central New Zealand (southern North Island and northwestern South Island; see Figure 3.1) by comparing the splitting measurements with $S_{H \max }$ from both gravitational stresses and tectonic stress (stresses at depth from focal mechanism inversion).

The structural-control hypothesis suggests that the orientation of the anisotropic medium is associated with the orientation of the geological structures (such as fault orientations and/or shear planes sub-parallel to faults) (Zinke, 2000; Boness and Zoback, 2006; Cochran et al., 2006; Okaya et al., 2016). Mineral alignment, associated with shearing in fault zones, or foliation in metamorphosed rocks has been one of the hypotheses used to explain structural-control anisotropy in the crust (Okaya et al., 1995; Godfrey et al., 2000). Around central New Zealand, Audoine et al. (2000) attributed the observed anisotropy to the metamorphosed schist in the crust because recrystal- 
lization during metamorphism may form schistosity or a pervasive foliation. Okaya et al. (1995) also observed a high degree (up to 20\%) of anisotropy associated with the Haast Schist of the South Island, New Zealand. Okaya et al. (2016) explained the crustal anisotropy in the Taiwan collision zone by deformation-related (due to pervasive shearing in strike-slip fault zones) mineral preferred orientation in the metamorphic rocks (e.g.; schist fabric). In this chapter, we examine these various hypotheses by comparing our splitting measurements with active fault orientations, orientations of horizontal principal stress (both tectonic and gravitational), and maximum shear plane orientations from the strain rate field (relation to crustal deformation).

\section{$3.2 \quad$ Tectonic setting and seismicity}

New Zealand's tectonic setting is characterized by two subduction systems of opposite polarity, connected by an area of oblique continental convergence (inset in Figure 3.1). Westward subduction (and intra-arc rifting) in the North Island at the Hikurangi subduction zone changes to strike-slip at the Marlborough Fault Zone, transpressional collision along the Alpine Fault and back to (eastward) subduction at the Puysegur Trench (inset in Figure 3.1) offshore (Mortimer, 2004).

The study area (red box on inset in Figure 3.1), central New Zealand, covers the southern end of the Hikurangi subduction zone and the northeastern part of New Zealand's South Island, which is dominated by the MFS. The MFS marks the transition from a subduction plate boundary in the north to a transpressive plate boundary (the Alpine Fault) in the south. The faults in the MFS are predominantly strike-slip (trending NE-SW parallel to the strike of the Pacific-Australia plate motion), with a relatively small component of reverse motion (Van Dissen and Yeats, 1991). The average regional strike of the faults is $055^{\circ}$ and their near-surface dips vary from $60^{\circ}$ to near-vertical (Van Dissen and Yeats, 1991; Anderson et al., 1993). Major geological structures around the southern end of the Hikurangi subduction zone strike in the NE-SW direction with active faulting also following this NE-SW trend (Mortimer, 2004; Litchfield et al., 2017). Southwest from Kaikōura lies the North Canterbury 


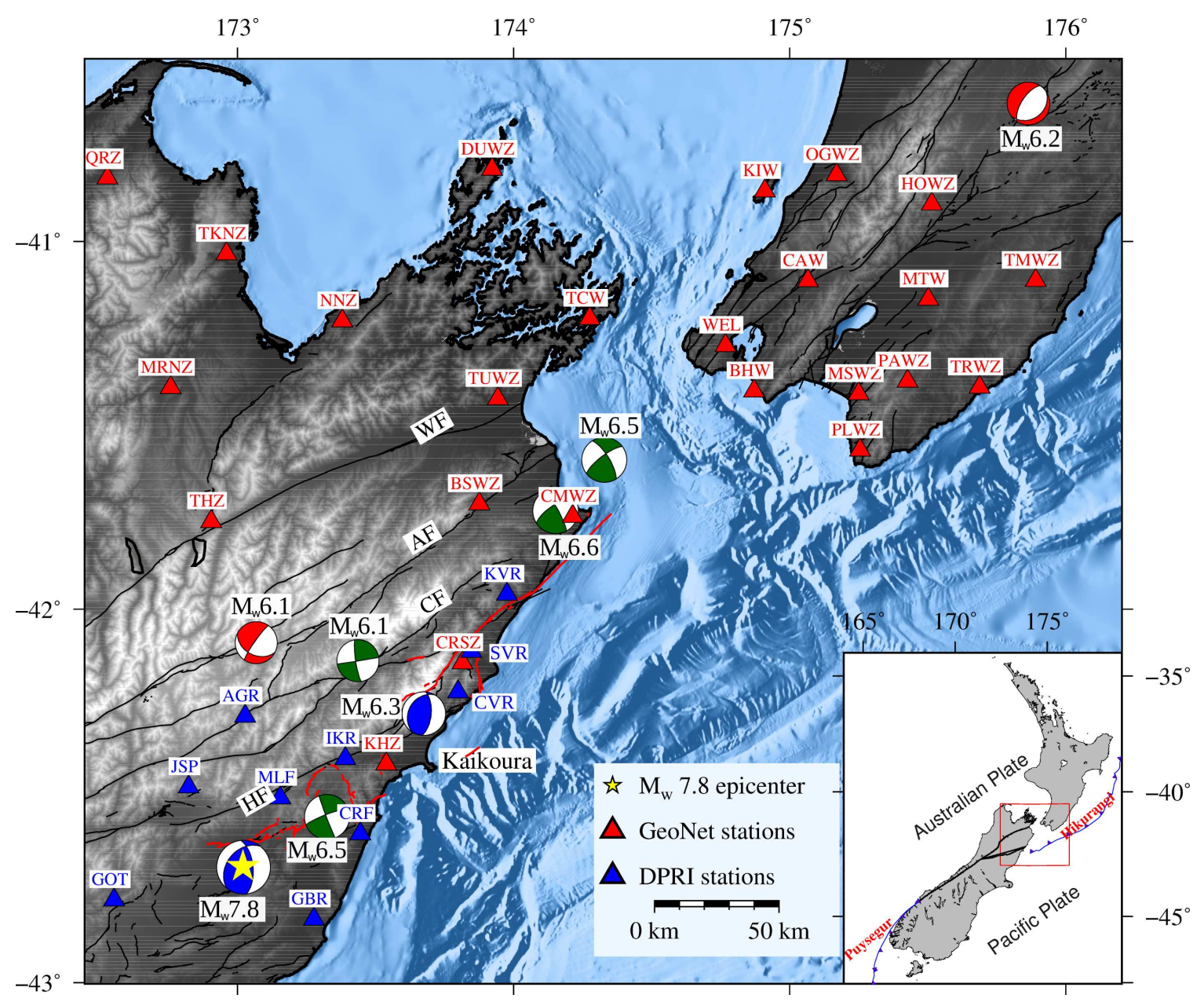

Figure 3.1: Map showing the tectonic setting of the study area with the stations used (red and blue triangles) plotted on a basemap of the digital topography and bathymetry (Mitchell et al., 2012). GeoNet CMT solutions of $M_{w}>6$ earthquakes from 2013 to 2018 are shown; blue focal mechanism plots (beach balls) represent reverse mechanisms, with the red representing normal faulting with some dip-slip motion, and the green represents a strike-slip mechanism. Major faults marked include the Wairau (WF), Awatere (AF), Clarence (CF) and Hope (HF) faults. The red lines denote the surface rupture of faults during Kaikōura mainshock (Langridge et al., 2016). The yellow star indicates the epicenter of the $M_{w} 7.8$ Kaikōura earthquake. Inset: The study region (red frame): the blue line is the plate boundary fault showing the Hikurangi and Puysegur trenches.

fold and thrust belt (Reyners and Cowan, 1993; Pettinga et al., 2001). The NE-SW trend of the thrust faults extends through the northeastern part of the Canterbury region. In response to the transition from the subduction-related tectonics in the north, thrust faults are evolving (Pettinga et al., 2001). The thrust faults are expressed as topographical ridges with NE-SW strike extending close to the Hope fault (Reyners and Cowan, 1993; Pettinga et al., 2001). Nearly two-thirds of the Marlborough region is 
underlain by greywacke of the Torlesse Supergroup, a structure with laterally uniform wave-speed (Reyners and Cowan, 1993; Mortimer, 2004). The Marlborough Schist, found north of the Wairau fault, which is part of the Haast Schist of South Island, is commonly considered to be strongly metamorphosed (Mortimer, 1993).

The 2013 Cook Strait earthquake sequence started in July 2013 with two foreshocks of $M_{w} 5.7$ and $M_{w} 5.8$ and climaxed in the July, $21^{\text {st }} 2013, M_{w} 6.5$ Seddon earthquake and the August, $16^{\text {th }}$ 2013, $M_{w}$ 6.6 Lake Grassmere earthquake (Figure 3.1). These large earthquakes, located $\sim 50 \mathrm{~km}$ south of Wellington, New Zealand's capital, generated significant ground shaking in the Wellington and Marlborough regions (Holden et al., 2013; Hamling et al., 2014). Both earthquakes were strike-slip events with similar magnitudes, epicentre, and source properties and they were considered to be a 'doublet' (Holden et al., 2013). The seismicity in the Cook Strait sequence region is still above the background levels that existed prior to 2013, with more than 16,000 earthquakes $\left(M_{L} \geq 1\right)$ within a 4 year period from January 2013 to November 2017 (Holden et al., 2013, and GeoNet catalog). Seismicity in Marlborough (before the 2016 Kaikōura earthquake) was mainly concentrated in the region above the subduction interface and around the north-eastern part of the MFS (Holden et al., 2013). The area was the location of a swarm of $M>4$ earthquakes in 2005 and before that, it was the location to the 1977 M 6 Cape Campbell earthquake and the 1966 M 5.8 Seddon earthquake (Holden et al., 2013). Six months after the $M_{w} 6.6$ Lake Grassmere earthquake, the $2014 M_{w} 6.2$ Eketahuna earthquake struck $10 \mathrm{~km}$ east of Eketahuna. The depth (34 $\mathrm{km}$ ) and location of the event placed it below the interface between the subducting Pacific plate and overriding Australian plate (Abercrombie et al., 2016). The afterhocks of the 2014 Eketahuna earthquake are characterized by repeating events (Wallace et al., 2017).

The $M_{w} 7.8,14$ November 2016, Kaikōura earthquake, is the largest and most complex earthquake recorded on land in New Zealand (Clark et al., 2017; Hamling et al., 2017). The earthquake initiated near the North Canterbury region at a depth of 15 $\mathrm{km}$ with an oblique thrust faulting mechanism (Hamling et al., 2017, Figure 3.1). The rupture propagated from south-west towards the north-east for about $120 \mathrm{~s}$, with an 
unusual source process, starting with weak radiation and releasing more energy while propagating towards the north-east over a distance of $\sim 150 \mathrm{~km}$ and terminating offshore in the Cook Strait region (Duputel and Rivera, 2017; Holden et al., 2017; Hamling et al., 2017; Cesca et al., 2017). A remarkable number (> 20) of shallow crustal fault segments (involving a combination of reverse and dextral strike-slip faulting; Figure 3.1) ruptured, including vertical motions of more than $10 \mathrm{~m}$ and horizontal displacements over $11 \mathrm{~m}$ (Clark et al., 2017; Hamling et al., 2017; Cesca et al., 2017). Most of the faults that ruptured have a general NE-SW trend (red lines in Figure 3.1), but some have NW-SE orientation, revealing a complex and heterogeneous slip pattern (Clark et al., 2017; Hamling et al., 2017; Kaiser et al., 2017). Surface rupture was mostly associated with known onshore faults. However, some surface traces were produced by faults that had not been previously mapped. Also some faults ruptured offshore, causing a small tsunami (6.9m "run up" was measured in Goose Bay, south of Kaikoura; Clark et al., 2017; Wallace et al., 2017). The 2016 Kaikōura earthquake was followed by more than 25,000 local and regional aftershocks (GeoNet catalog), clustered in three unique spatial patterns (Kaiser et al., 2017). More than 100,000 landslides were triggered by the earthquake and subsequent aftershocks, with 50 of them yielding significant landslide dams (Litchfield et al., 2017; Dellow et al., 2017). Another unusual aspect of the Kaikōura earthquake was the occurrence of large-scale $\left(>15,000 \mathrm{~km}^{2}\right)$ slow slip events triggered on the central and northern Hikurangi subduction interface (250 and $600 \mathrm{~km}$ away from the epicenter respectively) due to dynamic-stress changes from passing seismic waves (Wallace et al., 2017).

\subsection{Data and methodology}

\subsubsection{Data}

We determine SWS measurements for over 40,000 local crustal earthquakes that were located around the region of the 2013 Cook Strait earthquake sequence (Figure 3.2). Our earthquake catalog consists of GeoNet detection and locations (Petersen et al., 
2011) and spans more than 5 years: from January 2013 to June 2018. Although Lanza et al. (2019) relocated some of the events used here, we used the GeoNet catalog to ensure continuity and consistency. The GeoNet catalog used here has a good azimuthal coverage of events before and after the 2016 Kaikōura mainshock (supplementary Figure A.1). This enables us to search for temporal variations in SWS parameters and $V_{p} / V_{s}$ ratios.

We use 36 stations deployed around the Wellington and Marlborough region. These stations include 24 permanent seismic stations (combination of broadband and shortperiod instruments; red triangles on Figure 3.1) operated by GeoNet (the Geological hazard information for New Zealand) and 12 temporary short-period stations installed and operated by Disaster Prevention Research Institute, Kyoto University, Japan (DPRI) (Okada et al., 2019) at different time periods between 2009 and 2018 (blue triangles on Figure 3.1). Plots of data continuity for all stations are shown in supplementary Figure A.2. All the stations were sampled with a common sampling frequency of $100 \mathrm{~Hz}$. Since the objective was to study crustal anisotropy, the hypocentral depths were limited to less than $35 \mathrm{~km}$ to include only earthquakes whose ray paths travel wholly through the crust. Moreover, we limited events magnitudes to $M_{w} 1.5$ and above to remove any poor-quality waveforms.

\subsubsection{Method}

To estimate SWS parameters for thousands of waveforms, an automated and systematic technique was implemented. Here, we used the automatic splitting analysis code; Multiple Filter Automatic Splitting Technique, MFAST (Wessel, 2010; Savage et al., 2010a), which is designed to handle large volumes of data. MFAST is based on the eigenvalue-minimization method of Silver and Chan (1991) and the clustering method of Teanby et al. (2004a). MFAST uses an automated workflow to estimate the splitting parameters with an objective grading of measurements (Walsh et al., 2013). A concise description for MFAST is presented later in this section and a detailed description of the method is presented by (Savage et al., 2010a; Wessel, 2010; Walsh, 2012).

We used P-wave arrival times and origin times, $t_{o}$ determined by GeoNet (Petersen 

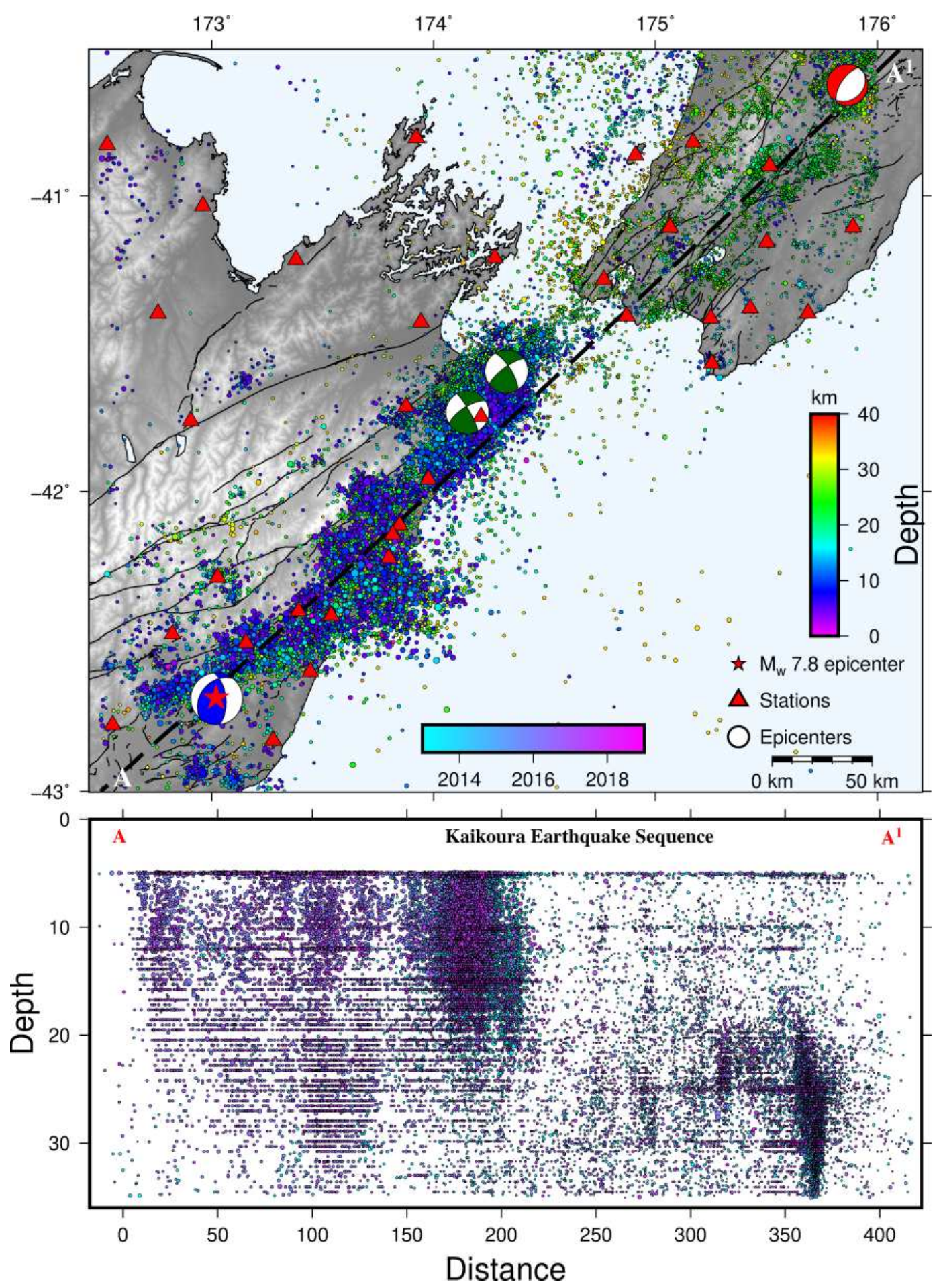

Figure 3.2: Map of earthquake epicenters used for analysis. Red triangles are the locations of GeoNet stations used. Events are colour-coded by the hypocentral depths. Blue focal mechanism solution (beach ball) represents the Kaikōura earthquake faulting mechanism; red beach ball represents the Eketahuna earthquake faulting mechanism, and green beach balls represent the two Cook Strait earthquake faulting mechanisms (Ristau, 2013). Focal mechanisms solutions were obtained from the GeoNet regional moment tensor solution catalog. A cross-section from $\mathrm{A}$ to $\mathrm{A}^{1}$ shows the projected depth distributions, coloured by time (time scale on the main Figure). 
et al., 2011). We used an automatic algorithm to estimate local shear wave arrival times for seismographs with good signal to noise ratio, SNR (SNR>3). All SWS measurements were made within the "shear wave window" (incidence angles less than a chosen critical angle, defined below). We used 1-D synthetic analysis (determining the effect of the angle of incidence on splitting measurements) to resolve the ultimate critical angle for our analysis (see Chapter 5 for details). This was necessary because, outside the shear wave window, the shear waveforms are susceptible to $\mathrm{S}$ to $\mathrm{P}$ conversions and scattering at the surface causing non-linear particle motion (Nuttli, 1961; Crampin and Gao, 2006; Savage et al., 2016). Neuberg and Pointer (2000) showed that waveforms outside the shear wave window generate elliptical particle motion even without the presence of anisotropy, especially when recording shallow local earthquakes in the vicinity of strong topography. Based on the testing done in Chapter 5, we selected a maximum angle of $35^{\circ}$ as the best shear wave window for our analysis. We estimated angles of incidence using the TauP toolkit (Crotwell et al., 1999) and a 1D velocity model extracted from the Eberhart-Phillips and Fry (2018) 3-D velocity model.

\section{Local phase arrival picking}

Determination of S-wave arrival times is necessary to estimate SWS measurements. Due to the large volume of our data set, manually picking the S-phase arrival times would have been too time-consuming. We, therefore, used two automatic picking techniques. First, local S-phase arrival times (S-picks) for GeoNet stations were automatically picked using the technique of Diehl et al. (2009) modified by Castellazzi et al. (2015), hereafter called Spicker. For stable and reliable S-wave picking, the Spicker technique combines three different detection and picking methods. The STA/LTA detector (e.g. Allen, 1978) and polarization detector (e.g. Flinn, 1965) are used to identify the first arriving S-phase. The information provided by the detectors is then used to set up the search windows of the autoregressive picker using the Akaike Information Criterion (AR-AIC) as outlined by Takanami and Kitagawa (1988). Finally, all three methods are combined to yield the best arrival-time of the first arriving S-phase and its corresponding uncertainty. The Spicker makes use of all the three-component seis- 
mograms to estimate the S-phase arrival. See Diehl et al. (2009) and Castellazzi et al. (2015) for further details on the algorithm.
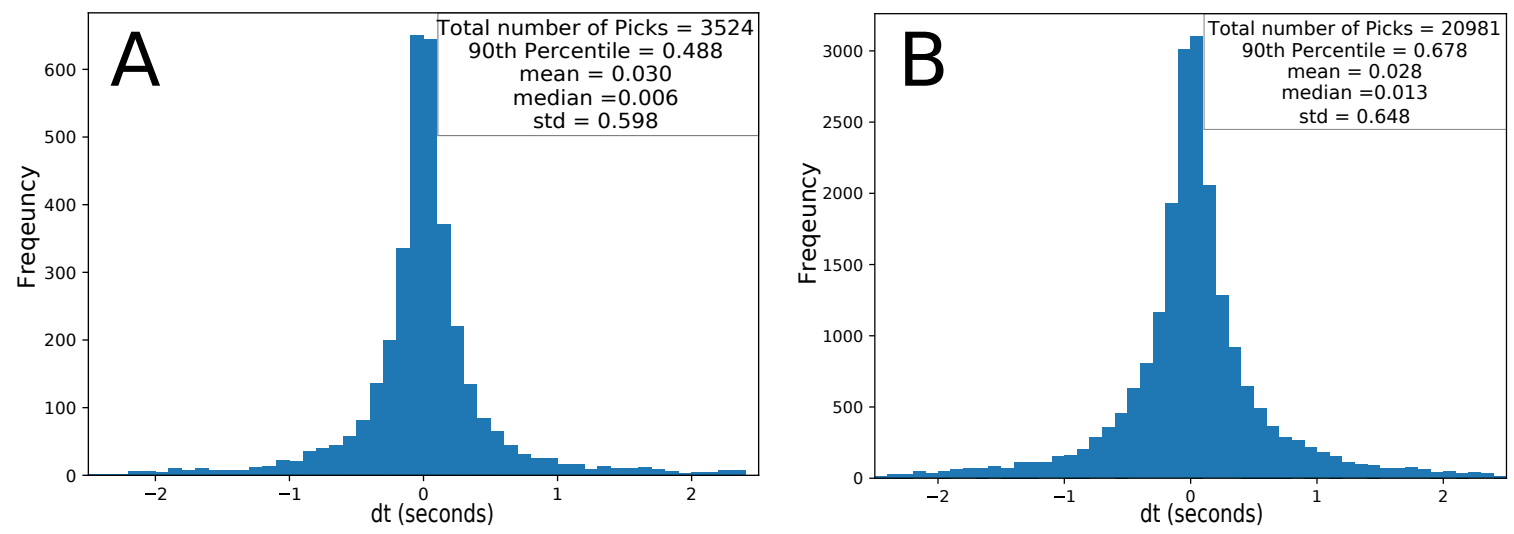

Figure 3.3: Distributions of local S-phase picking errors relative to analyst pick. A: Time difference between Spicker picks and manually picked S-phase arrival times. B: Time difference between GeoNet manual picks and Spicker picks.

We calibrated the picking algorithm with over 3,500 manually picked S-phases. These S-phases were randomly selected to obtain a uniform station, magnitude, and depth distribution. The time difference between manual and Spicker picks revealed a symmetrical uni-modal distribution with a mean of $0.03 \mathrm{~s}$ and a standard deviation of 0.598 s. In addition, $90 \%$ percent of the Spicker picks were within \pm 0.484 s of the manual S-picks (Figure 3.3A). This picking accuracy is adequate for our studies because MFAST uses multiple time windows for its analyses. The best parameters (Table A.1) that yielded more picks and the small-time differences between manual and Spicker picks were used for the final picking. We compared the common S-picks ( 21,000) between GeoNet manual S-picks and Spicker picks across all stations. The time difference between GeoNet manual S-picks and Spicker picks revealed a symmetric unimodal distribution with mean $0.028 \mathrm{~s}$, standard deviation $0.648 \mathrm{~s}$, and $90 \%$ of the picks were within \pm 0.678 s (Figure 3.3B). Over 200,000 S-phases were obtained, representing about $23 \%$ of the expected (event-station pair) arrivals. Moreover, to assess the quality of the S-picks across our dataset, we randomly selected approximately $5 \%$ of the S-picks across all stations. The selected events were manually inspected and 
approximately $15 \%$ of S-picks were unreliable and were either revised or discarded. Based on this analysis we estimate approximately $85 \%$ of the S-picks are reliable.

Some of components of the short period stations operated by DPRI were intermittently missing components. We could not apply the Spicker technique to estimate the S-phase arrival since the technique required all three components for the estimation. Instead, we used the Generalized Seismic Phase Detection, GPD, a technique developed by Ross et al. (2018) for estimating phase arrivals for the DPRI stations. This technique trained a Convolutional Neural Network (a deep learning framework) to learn the features of seismic waveforms. An already trained model can be applied to waveforms that are not included in the training set, thus a trained model can be applied to different tectonic settings (Ross et al., 2018; Kong et al., 2018). We used the Ross et al. (2018) GPD-framework model which they trained and validated with a total of 4.5 million three-component waveforms across a range of magnitudes. With each component's time series, the model predicts the probability that a phase arrival is either a $\mathrm{P}$ or $\mathrm{S}$ arrival. We set the minimum probability for phase detection to $95 \%$. An example of a pick example is shown in supplementary Figure A.3. To gain confidence in the GDP picks, we estimated arrival times for events with GeoNet manual S-picks (more than 40,000 picks across the 24 permanent GeoNet stations) and compared the time difference between the GDP S-picks and GeoNet manual S-picks. The time difference shows a concentrated symmetric uni-modal distribution with mean $-0.072 \mathrm{~s}$, standard deviation $0.774 \mathrm{~s}$, and $90 \%$ percent of the picks were within $\pm 0.261 \mathrm{~s}$ (See supplementary Figure A.4).

\section{Measuring splitting parameters}

SWS parameters were estimated using the MFAST code (Wessel, 2010; Savage et al., 2010a). This procedure finds the inverse splitting operator that best removes the splitting. The waveforms are filtered with a series of band-pass filters, and the product of the bandwidth and the signal-to-noise ratio of the filtered waveforms is used to determine the best three filters. Splitting measurements for all three filtered waveforms are estimated using the Silver and Chan (1991) and Teanby et al. (2004b) method with 
modification by Walsh et al. (2013). The splitting parameters are estimated using the eigenvalue minimization method repeated over multiple time windows around the Sphase. The splitting parameter pair $(\phi, \delta t)$ that best removes the splitting as measured by the smallest eigenvalue of the corrected covariance matrix, is chosen as the best measurement for the given time window (Savage et al., 2010a).

This procedure is then repeated for 75 windows covering slightly different time spans around the S-phase. The windows are automatically selected based on the dominant period around the filtered shear wave (Savage et al., 2010a). Cluster analysis over the 75 window measurements is used to select the final splitting parameter for the filter under consideration and to calculate the uncertainty associated with the measurements (Teanby et al., 2004a). Measurements are graded from A to D depending on the consistency between the $(\phi, \delta t)$ and measurements in the different windows. MFAST also provides a measure of the incoming polarization orientation $\left(\phi_{i n}\right)$ by estimating the eigenvalues of the corrected components after splitting is removed (Silver and Chan, 1991; Savage et al., 2010a). $\phi_{\text {in }}$ corresponds to the polarization orientation of the shear wave before it enters the anisotropic layer responsible for the measured splitting parameters. As a quality control, we removed all null graded measurements (events for which no measurable splitting occurs or event for which $\phi$ is within $20^{\circ}$ of parallel or perpendicular to $\phi_{i n}$ ) and also kept only A and B graded measurements. We also limited maximum delay times, $\delta t$, to $0.4 \mathrm{~s}$ since local crustal events are often not expected to have larger delay times (Balfour et al., 2005; Cochran and Kroll, 2015). Following the quality control, a total of 102,260 (out of 398,169) high-quality measurements were obtained. Wessel (2010), Savage et al. (2010a) and Walsh et al. (2013) give a detailed description of the MFAST method.

We estimated $V_{p} / V_{s}$ ratios along the ray path of each event that yielded high-quality SWS measurements to each station using the approach of Wadati and Oki (1933) and Nur (1971). We assumed a linear ray path and that the $V_{p} / V_{s}$ ratio is homogeneous along the seismic wave's path (Kisslinger and Engdahl, 1973). The $V_{p} / V_{s}$ ratio of each event station pair was calculated using $V_{p} / V_{s}=\left(t_{s}-t_{o}\right) /\left(t_{p}-t_{o}\right)$, where $t_{s}$ and $t_{p}$ are the arrival times of the $\mathrm{S}$ and $\mathrm{P}$ waves respectively, and $t_{o}$ is the event origin time (from 
the GeoNet catalog). Only $V_{p} / V_{s}$ values between 1.5 and 2.3 were used because values outside of this range are not expected around the study region (Eberhart-Phillips and Fry, 2018) and would probably be due to inaccurate arrival time picks.

We also estimated the percent anisotropy, $\kappa$, following the approach of Babuska and Cara (1991) and Savage (1999). Here we define $\kappa=\left(v_{\max }-v_{\min }\right) / \bar{v}$. Where $v_{\max }$ is velocity of the shear wave along the fast orientation $v_{\min }$ velocity of the shear wave along the slow orientation and $\bar{v}$ is an average of $v_{\max }$ and $v_{\min }$. Assuming both fast and slow waves travel with the same path length, $\kappa$ can be related to $\delta t$ as: $\kappa=200 \times \delta t /\left(2 t_{s}+\delta t\right)$. We use $\kappa$ as well as $\delta t$, to characterize the anisotropy structure.

\section{Spatial averaging}

SWS measurements provide an estimate of the anisotropy along the ray propagation path between the source and station. However, it can be difficult to determine exactly where along the ray propagation path the anisotropy originates. With measurements from several stations and a dense cluster of events, one can probe the spatial variation of $\phi$ and $\delta t$ using a spatial averaging technique. We used the Tomography Estimate and Shear wave splitting Spatial Average (TESSA) technique developed by Johnson et al. (2011) to estimate the spatial averages of $\phi$.

For the spatial averaging technique, the study area is divided into cells or blocks using the recursive quadtree clustering algorithm described by Townend and Zoback (2001). We set the minimum block size to $5 \mathrm{~km}^{2}$ and the minimum and a maximum number of raypaths to be 20 and 80, respectively. The quadtree gridding algorithm works in an iterative process. Cells with fewer than 20 rays passing through are not used for analysis and those with more than 80 rays passing through are subdivided until a minimum block size of $5 \mathrm{~km}^{2}$ or a minimum number of 20 raypaths is attained. These criteria were chosen to ensure that each block contained enough data to give a reliable average measurement. To take into account variations due to heterogeneous anisotropic structure along the raypath (e.g. Johnson et al., 2011; Rümpker and Silver, 1998), rays in each grid cell are weighted by the inverse of the distance squared $\left(1 / d^{2}\right)$ (where $d$ is the distance from the station to the grid cell in question). This weighting 
scheme is used because we expect splitting to occur later in the ray-path (closer to the surface) and we did not observe a strong correlation between $\delta t$ and hypocentral depth (Johnson et al., 2011). For comparisons with time, we used the regular gridding (not the quad-tree gridding) to ensure that the data points are always at the same locations. The spatial averages of $\phi$ are computed using a circular statistics approach (Berens, 2009) and are estimated only when the standard deviation of fast orientations in each grid is less than $30^{\circ}$ and the standard error of the mean is less than $10^{\circ}$.

\section{Quantitative comparison of $\phi$ measurements}

We made quantitative comparisons of our spatially averaged $\phi$ measurements with GNSS derived principal contraction axes, average fault orientations, gravitational stress and focal mechanism inversion measurements. We also quantitatively compared averaged $\phi$ measurements between events before and after the 2016 Kaikōura earthquake to observe any spatio-temporal variation associated with the 2016 Kaikōura mainshock. For our quantitative comparison, we estimate a test statistic, $F$, the absolute value of the cosine of the difference between the two angles $\left(F=\left|\cos \left(\psi_{1}-\psi_{2}\right)\right|\right)$ where $\psi_{1}$ is the mean $\phi$ measurement and $\psi_{2}$ is the comparison angle. Since we do not have an identical set of locations at which mean $\phi$ has been estimated, we use locations that are closest together, and $<10 \mathrm{~km}$ to estimate the residual. Values of $F$ range from 0 to 1 , where 1 represents parallel orientation and 0 represents perpendicular orientation between $\psi_{1}$ and $\psi_{2}$. We contour the residual using the GMT functions grdsample and grdimage (Wessel et al., 2013). We also estimate the mean and median of the $F$ to give an indication of how well the two angles are correlated.

\section{Averaging for time variations}

To examine variations, we employed an averaging technique for values of $\delta t$ to smooth and minimize the scatter as much as possible. We use a simple moving median as the smoothing function that reduces the noise in the measurement. We used a 20-day moving median filter on the individual results with the aim of removing the scattered values from the data set. We estimate the $95 \%$ confidence intervals by using 
the non-parametric bootstrap approach. In this approach, we randomly sample with replacement from the original sample a dataset of the same size as the original sample. We repeat this for 1000 replicates and for each replicate dataset we estimate the median value. The 2.5 th and 97.5 th percentile of the replicate dataset is estimated as the respective lower and upper values of the $95 \%$ confidence interval for the median.

\subsection{Results}

We determined SWS measurements for more than 40,000 waveforms recorded on at least one of the 36 stations within the study region (Figure 3.1). Tables $3.1 \& 3.2$ give a summary of fast orientations, delay times, percentage of anisotropy and $V_{p} / V_{s}$ ratio, as well as associated descriptive statistics for each station. Out of the $\sim 102,000$ high-quality SWS parameters we estimated, the delay times vary between near zero to $0.372 \mathrm{~s}$ with an average of $0.157 \pm 0.001 \mathrm{~s}$. The $V_{p} / V_{s}$ ratio varies from 1.58 to 2.22 with an average of $1.741 \pm 0.001$. Percent anisotropy varies from near 0 to $5.186 \%$ with an average of $0.922 \pm 0.004 \%$.

Rose diagrams of $\phi$ orientations recorded at each station, overlain on a basemap of the digital topography, are shown in Figure 3.4. $S_{H \max }$ orientations from Townend et al. (2012) and Balfour et al. (2005) are also shown as green bow-ties with wedges showing the $95 \%$ confidence interval. $\phi$ orientations for most of the stations show a dominant NE-SW orientation, which is parallel to the strike of the geological structures in the region. A few stations show NW-SE orientations.

Around the MFS, $\phi$ orientations are generally parallel to the strike of the active faults. The consistent trend suggests a relation between $\phi$ and the structural trend around the central and western MFS. Yet some stations, KHZ, IKR, CRF, and GBR, at the eastern coastal edge of the MFS yield NW-SE orientations of $\phi$, which is perpendicular to the structural trend and sub-parallel to the crustal $S_{H \max }$ orientations. At station CMWZ and KVR, we observe a bi-modal distribution of $\phi$ orientations, with one subset sub-parallel to the $S_{H \max }$ orientation and the other subset sub-parallel to the strike of the faults. At stations BSWZ and TUWZ, $\phi$ orientations are sub- 


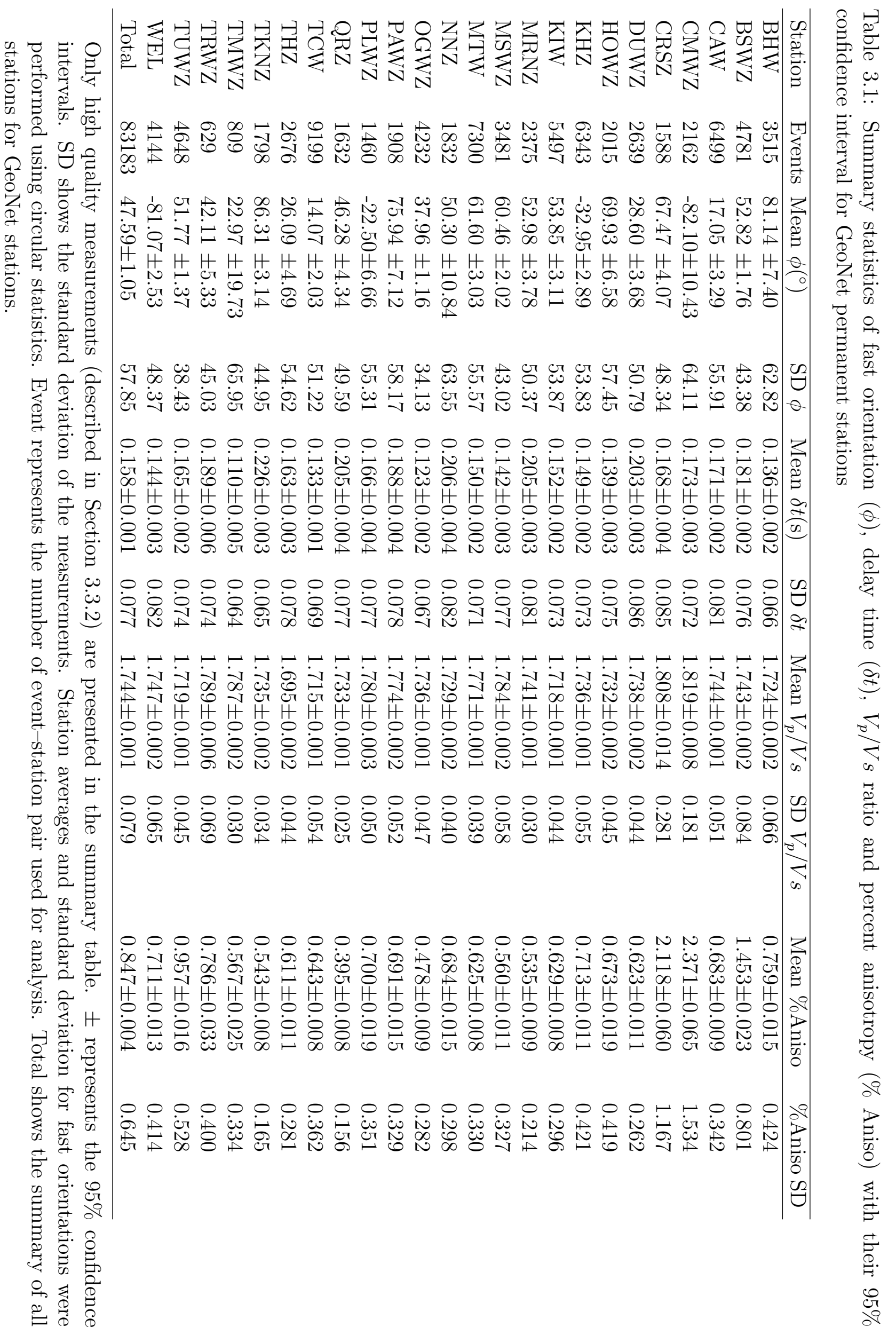




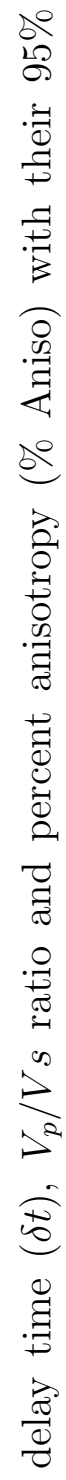

是

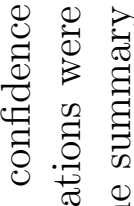

be 晃

๙2.

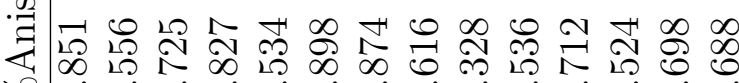

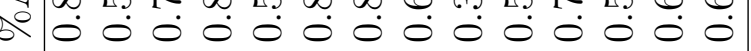

1.

毘

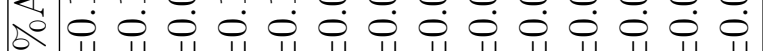

đี

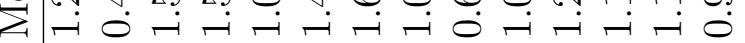

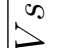

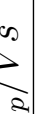

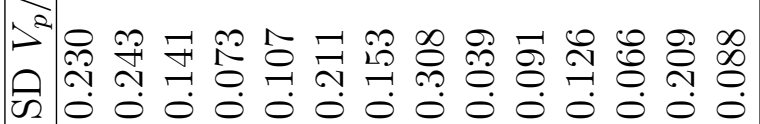

๓ ำร

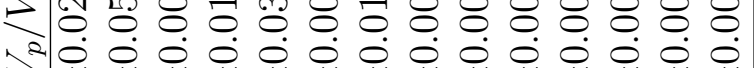

$\lambda^{2}$ H $0^{H}$ H

שี

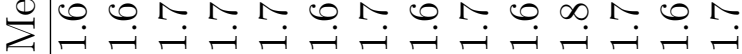

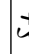

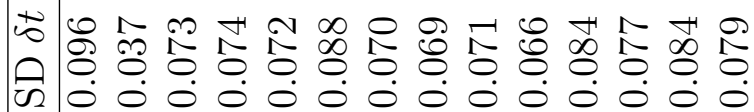

Q

()

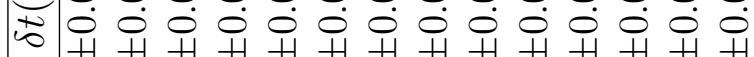

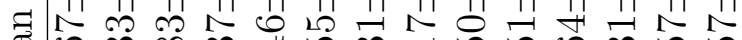

ธี

票

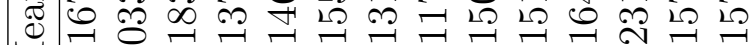

乙

Q

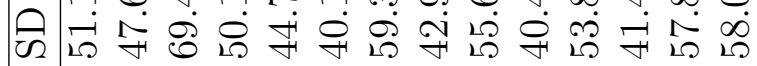

茪

$\rightarrow$

$+$

पै

용

蔼

20.

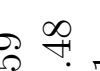

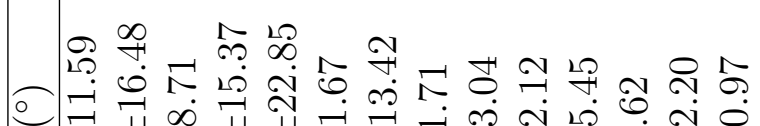

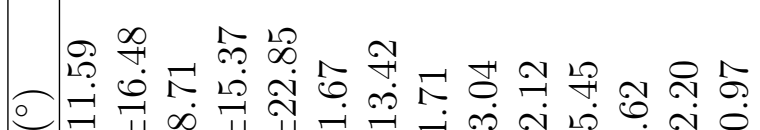

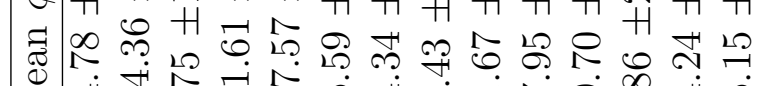

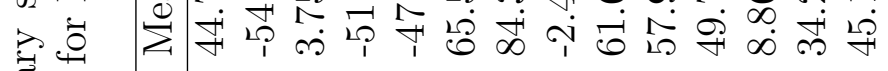

疍

$\ddot{\sim} \underset{0}{\ddot{0}}$

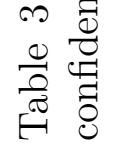

(1)

南共

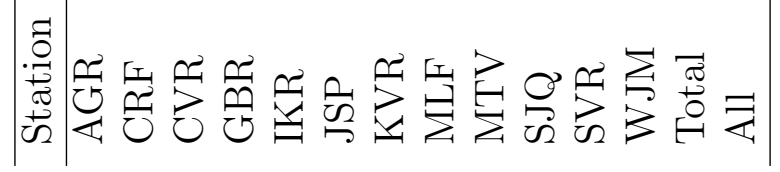

蛋

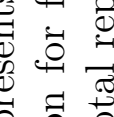

苞

$+\frac{\pi}{\pi}$

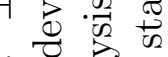

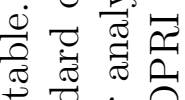

ت्ञ

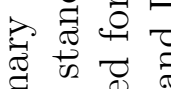

寻荥

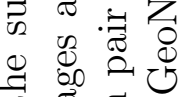

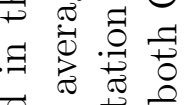

鄫

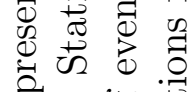

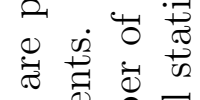

ลิ

ه

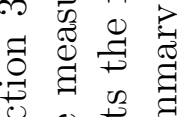

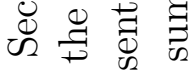

.

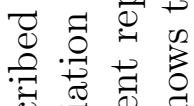

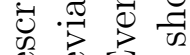

过导闵专

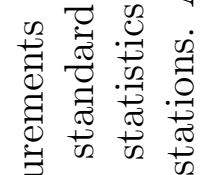

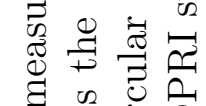

氙

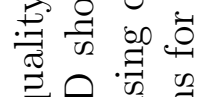

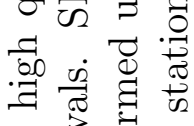

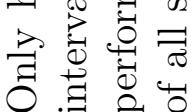


parallel to the orientation of the nearby Awatere and Wairau Faults respectively and perpendicular to the nearby $S_{H \max }$ orientation.

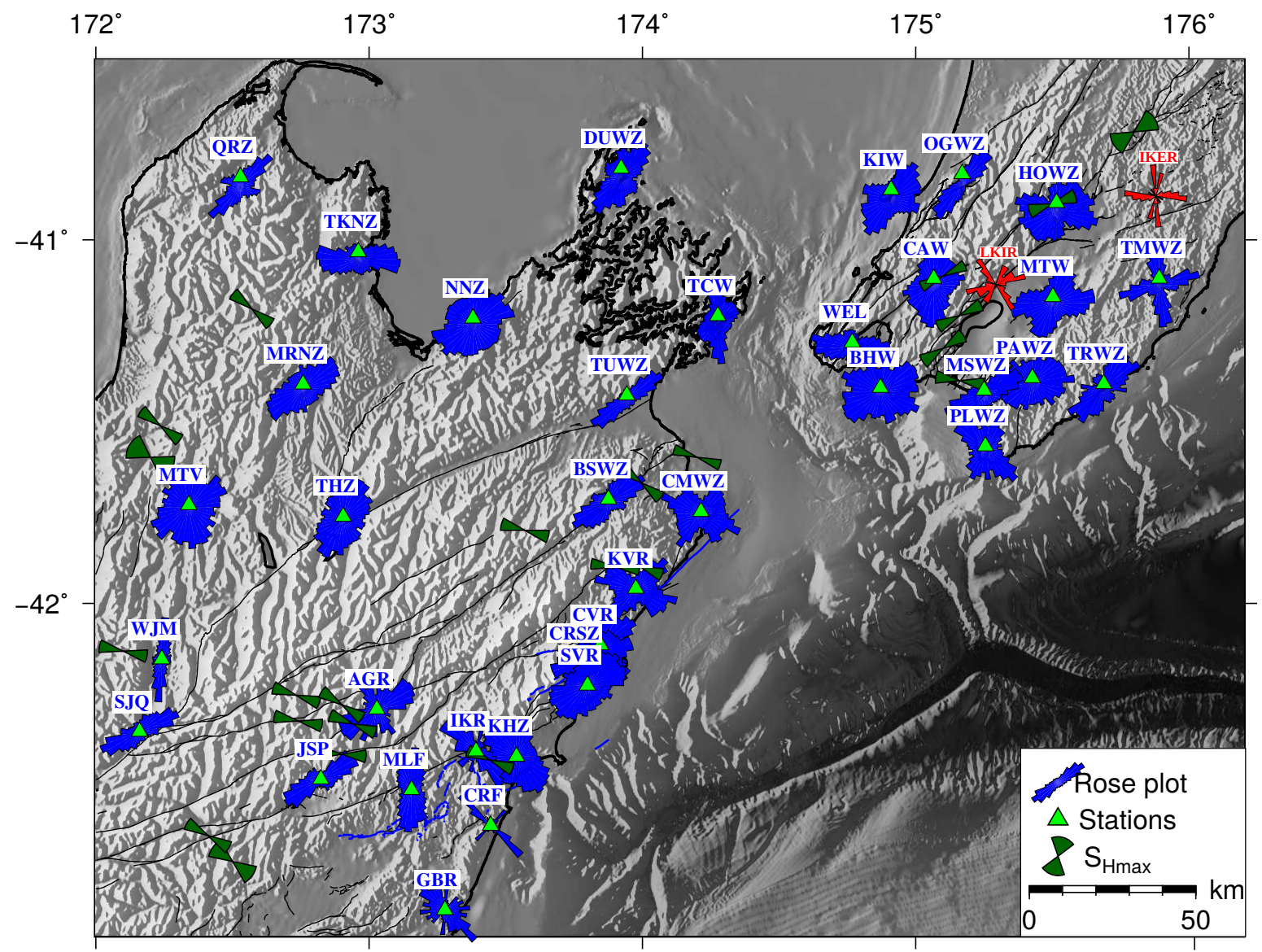

Figure 3.4: Rose diagrams (circular histograms) showing the $\phi$ orientation results from local S-phase events, $\phi$. Rose diagrams are plotted on the stations at which measurements were made with a basemap of the digital topography and bathymetry. The green bow-ties shows the crustal $S_{H \max }$ orientations from Townend et al. (2012) and Balfour et al. (2005) with wedges showing the $95 \%$ confidence interval. The black lines are the active faults. The two red rose diagrams are measurements from Audoine et al. (2000) showing bi-modal distributions. The blue lines are the faults that ruptured during the Kaikōura Earthquake (Langridge et al., 2016).

Around the southern end of the North Island, the $\phi$ orientation at most stations exhibit significant variations from each other. Some stations exhibit a bi-modal distribution while others show a dominant NE-SW direction, with a few stations exhibiting trends that are neither parallel to $S_{H \max }$ nor to the strike the major faults. At stations TMWZ and MTW, a clear bi-modal distribution of fast polarization orientation is observed. One subset trends NE-SW and the other subset trends NW-SE (Figure $3.4)$. 
To investigate temporal variations resulting from the 2016 Kaikōura mainshock, $\phi$ measurements were split into two subsets: before (from 01/01/13 to 13/11/16) and after (from 14/11/16 to 30/06/18) the Kaikōura mainshock. The 2013 Cook Strait earthquake sequence produced ample seismicity to determine the background $\phi$ orientation prior to the 2016 Kaikōura mainshock. Figure 3.5 shows rose diagrams of $\phi$ measurements before (Figure 3.5A) and after the 2016 Kaikōura mainshock (Figure $3.5 \mathrm{~B})$.

\subsubsection{Spatial averaging}

Using the technique described in Section 3.3.2, we performed spatial averaging analysis for more than 102,260 source-receiver $\phi$ measurements. Based on the criteria outlined in Section 3.3.2, 4,579 out of 4,720 grid elements created were used for analysis (see supplementary Figure A.5). Circular averages of $\phi$ measurements in each grid square are represented by the bar (coloured by mean $\phi$ value) and plotted at the center of each grid square (Figure 3.6). The spatial averaging results show a significant spatial variation. We observe a dominant NE-SW (see yellow bars on Figure 3.6) trend which is sub-parallel to the strike of the major active faults and also the topographic trends. We also note a clear contrast of perpendicular fast orientations (blue bars on Figure 3.6) around the eastern end of the MFS (the region that ruptured during the Kaikōura earthquake). This NW-SE feature existed before the 2016 Kaikōura sequence (see supplementary Figure A.6).

To investigate the spatio-temporal variation of $\phi$ measurements associated with the 2016 Kaikōura mainshock we performed spatial averaging analysis for events before and after the earthquake. Using the method described in Section 3.3.2, we made quantitative comparisons of our spatially averaged $\phi$ measurements between events before and after the 2016 Kaikōura mainshock to search for potential spatio-temporal variations associated with the 2016 Kaikōura mainshock. The orientations of spatially averaged results for before (green bars) and after (blue bars) are shown in Figure 3.7. Generally, the orientations of the two angles are in agreement which indicates no significant spatial-temporal variation (in most cases the blue after-bar is invisible under 

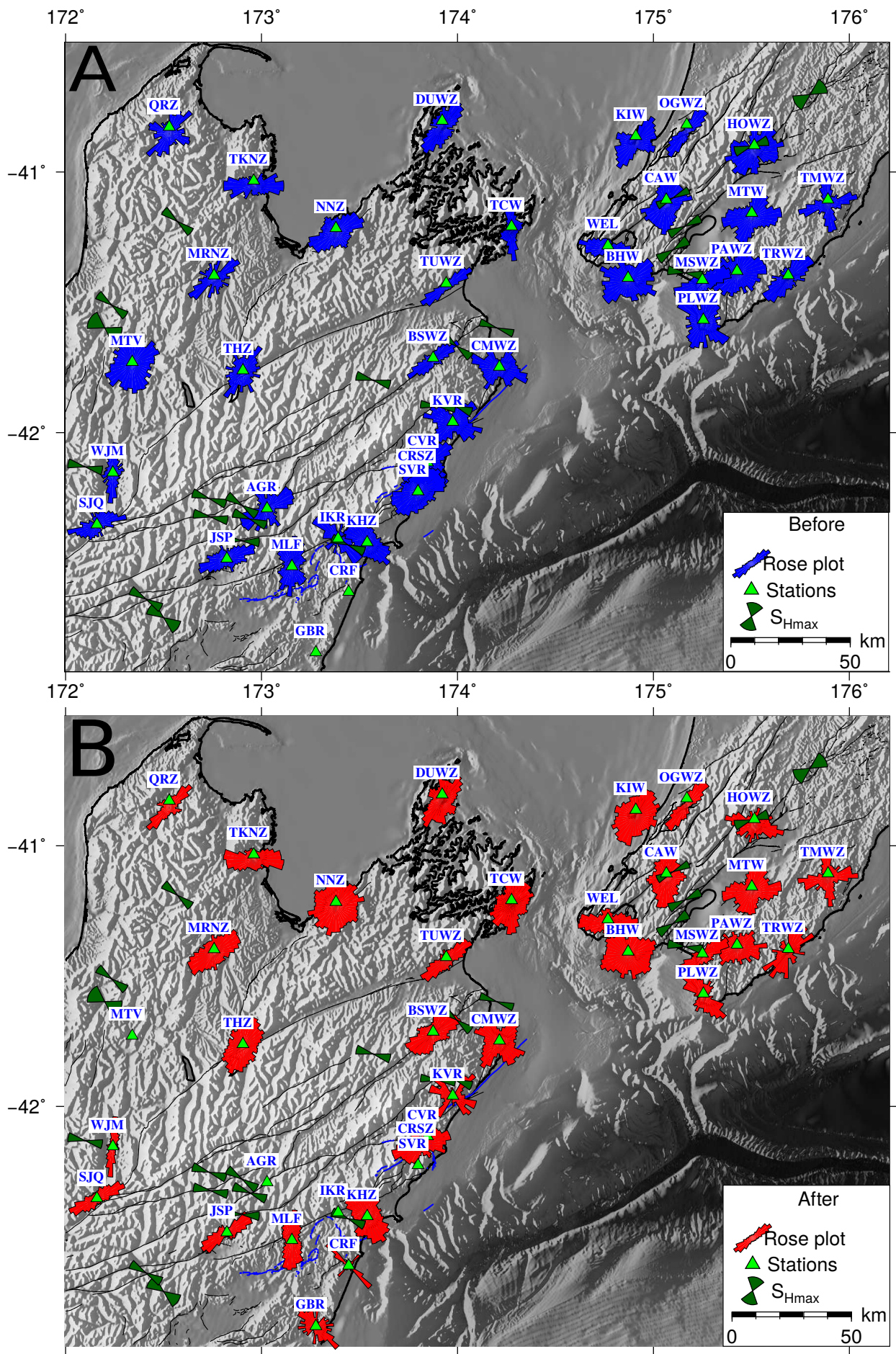

Figure 3.5: SWS fast orientation results from local events for before, A (from 01/01/13 to $13 / 11 / 16$ ) and after, B (from 14/11/16 to 30/06/18) Kaikōura mainshock. The $\phi$ orientation determined from events recorded at each station are represented as rose diagrams and plotted on a basemap of the digital topography. Rose diagrams are plotted on the stations at which measurements were made. The green bow-ties shows the $S_{H \max }$ orientations from Townend et al. (2012) and Balfour et al. (2005) with wedges showing the $95 \%$ confidence interval. The blue lines are the faults that ruptured during the Kaikōura Earthquake (Langridge et al., 2016). 


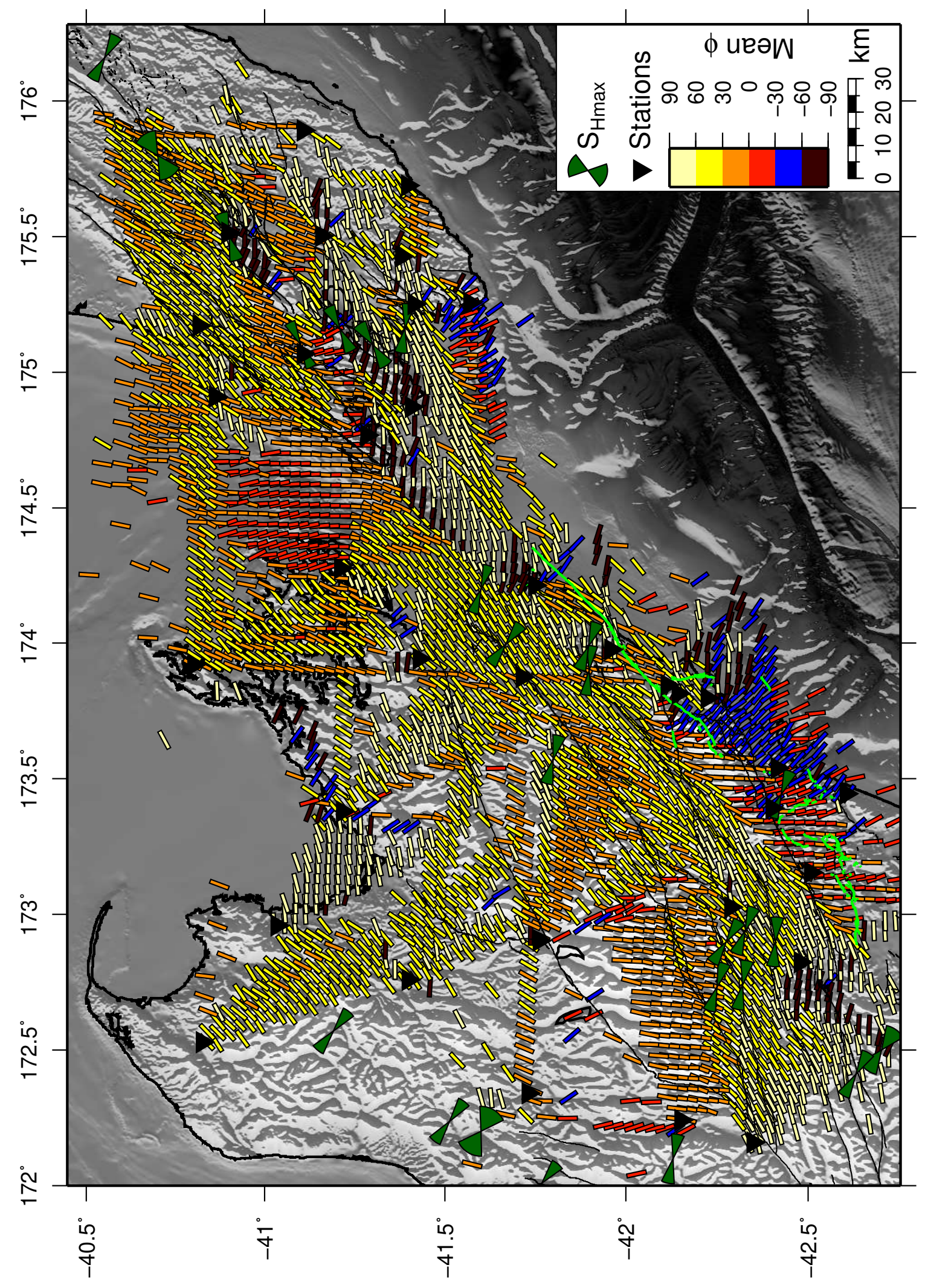

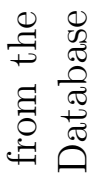

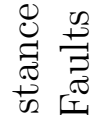

용

옹

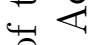

N

学

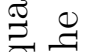

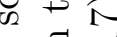

콩

$\therefore$ is

สี

范

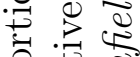

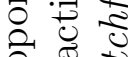

疍

要过

要

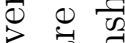

$\exists \approx$.

so

$\exists$

$\frac{9}{60} \div$

远需

둔

萡。

ส

. 워

$\pi$

․․ㅇ.

항

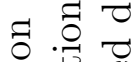

若

김

స్ㅠ

过著

때

पे

(

80

38

๘

.]ల్

营,

थ 0

అृ

ले

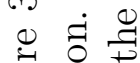

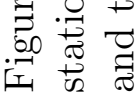


the exactly matching green before-bar). The mean and median values of $F$ (Section 3.3.2) are $0.92 \pm 0.011$ and 0.99 respectively (thus a mean and median difference of $23^{\circ}$ and $10^{\circ}$ respectively), indicating a strong agreement between the orientations before and after the mainshock. Out of the 898 co-located measurements, $88 \%$ of them had a test statistic value, $F$, above $0.8\left(37^{\circ}\right)$ with only $1 \%$ less than $0.2\left(78^{\circ}\right)$ (Figure 3.7B). The general agreement observed, also seen from the scatter plot (Figure 3.7C) of before and after measurements, suggests that there is no significant temporal variation associated with the 2016 Kaikōura earthquake. Although we observe a few patches of disagreement around the propagation path of the 2016 Kaikoura mainshock, we attribute this to spatial variation rather than temporal variation since ray paths for after measurements are slightly different from before measurements (see supplementary Figure A.6). The red patch at the north-eastern edge of the study region may be due to an edge effect from the grid. Due to limited station coverage off-shore, we limit our discussion to onshore results.

\subsection{Discussion}

\subsubsection{Overall station trends}

The observed delay times, varying between near zero to $0.372 \mathrm{~s}$, are in agreement with other SWS studies of crustal earthquakes (Audoine et al., 2000; Balfour et al., 2005; Crampin et al., 2015; Cochran and Kroll, 2015; Savage et al., 2016). $V_{p} / V_{s}$ ratios varying from 1.58 to 2.22 with an average of $1.746 \pm 0.001$ are also consistent with tomography results from Eberhart-Phillips and Fry (2018) and close to the global average of 1.76 for continental crust (Christensen, 1996). There is, however, substantial variability in $\phi$ measurements (the standard deviation of the $\phi$ measurements is greater than $30^{\circ}$ at most stations). The observed large scatter of $\phi$ measurements suggests that the source of anisotropy may be a combination of more than one mechanism or that the mechanism varies rapidly spatially (Peng and Ben-Zion, 2004). The significant spatial variation we observe illustrates the complex regional tectonics and heterogeneous 


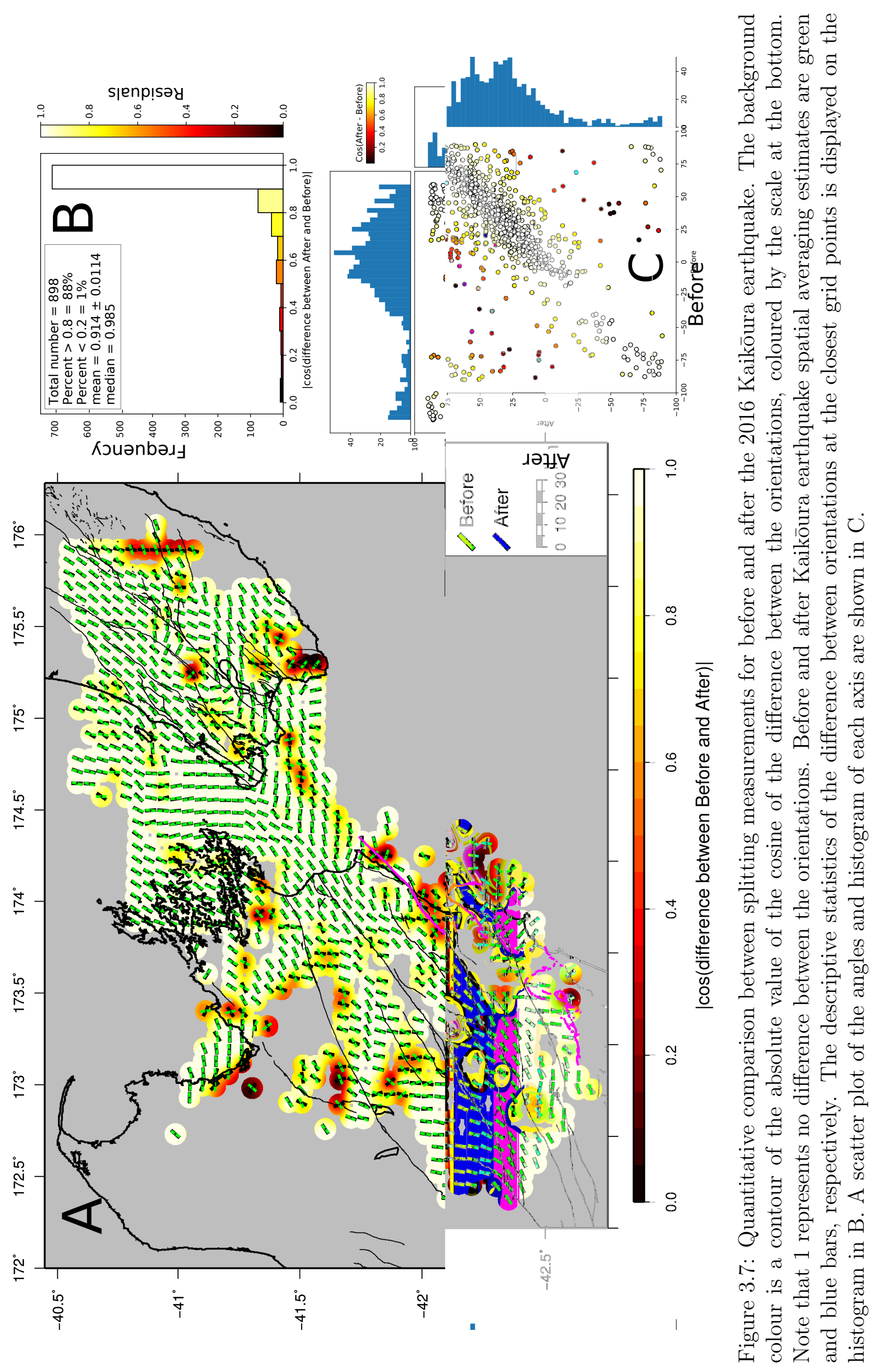


structures around central New Zealand.

The average $\delta t$ we observed (Table 3.1 and 3.2) is about $6-14$ percent of the values ( 1.6 s) reported from deeper anisotropic studies using telesiesmic data around New Zealand (Klosko et al., 1999; Zietlow et al., 2014). This small value in $\delta t$ suggests that there is a deeper source of anisotropy beneath these stations that are measured by SKS-phases and our measurement sees shallow anisotropy. A similar conclusion has been reached by previous studies (Audoine et al., 2000; Karalliyadda and Savage, 2013) where they reported delay times for SKS measurements far greater than the observed local S-phase measurements. Similarly, most studies of SKS waves assume the mantle is the main source of anisotropy (e.g. Savage, 1999).

The bi-modal distribution of fast orientation measurements observed at stations TMWZ and MTW on the North Island (Figure 3.4) reinforces previous observations. Audoine et al. (2000) reported a similar bi-modal distribution of $\phi$ measurements at stations LKER and LKIR (red rose diagram on Figure 3.4) which were in close proximity to TMWZ and MTW. At station HOWZ, the dominant $\phi$ orientation is parallel to faulting in the area and also sub-parallel to $S_{H \max }$. The correlation between $\phi$ orientation and the orientation of the active faults at these stations suggests that the sources of anisotropy beneath them could be structurally controlled. However, the faults are also parallel to the strike of the topography, which is consistent with nearsurface gravitational stress. Fast orientations at stations WEL and TCW (on either side of the Cook Strait; Figure 3.4) are consistent with recent studies (Balfour et al., 2005; Evanzia et al., 2017) around the Marlborough and Wellington region. However, they show neither an agreement with $S_{H \max }$ orientations nor with tectonic structures in the region.

Around the Marlborough region, the faulting is mainly strike-slip, thus, based on Anderson's theory of faulting (Anderson, 1905; Healy et al., 2012), we expect the faults to generally strike at an angle of $\sim 30^{\circ}$ to the orientation of $S_{H \max }$. Stations KHZ, IKR, CRF and GBR (and subset of $\phi$ measurements at stations KVR and CMWZ), located around the eastern end of the MFS have $\phi$ orientations that are sub-parallel to the orientation of $S_{H \max }$ rather than the fault fabric, suggesting stress-aligned micro-cracks 
(stress-controlled anisotropy) exist (Figure 3.4 and 3.5). However, at these stations, the role of structural anisotropy cannot be completely ruled out since there are subsets of the $\phi$ measurements that are sub-parallel to the major structural features.

An abrupt change from NE-SW to a NW-SE trend of the averaged fast orientation (blue bars on Figure 3.6) is observed at the edge of the high irregular topography boundary (the Kaikōura ranges and the Kaikōura canyon) around the eastern end of the MFS. This suggests that gravitational stresses could be contributing to the source of anisotropy around this region. In Section 3.5.5 we quantitatively compare averaged $\phi$ to the orientation of $S_{H_{\max }}^{G r a v}$.

\subsubsection{Search for temporal variation}

The use of temporal variation in SWS measurements to monitor precursory changes preceding major earthquakes is controversial (e.g. Aster et al., 1990; Crampin, 1990a; Crampin et al., 2015). There have been studies that have used temporal variations to monitor coseismic stress changes (Saiga et al., 2003) and post-seismic fault healing after major earthquakes (Tadokoro et al., 1999; Tadokoro and Ando, 2002). Around the central Apennines of Italy Piccinini et al. (2006) reported temporal variations in anisotropic parameters during the days before and after the occurrence of the 1997 Umbria-Marche seismic sequence. They suggested that the temporal variation was caused by the changes in local stress conditions and fluid pressure. However, they concluded that spatial sampling of the selected ray paths may vary with time and the possible contribution of spatial variations of anisotropic parameters can not be excluded. Lucente et al. (2010) also observed a clear temporal variation of seismic anisotropy and velocities before the $2009 M_{w} 6.3$ L'Aquila earthquake, Italy. They inferred that fluids, related to dilatancy-diffusion processes, played a key role in the fault failure process. In southern California, Yang et al. (2011) searched for possible temporal variations of crustal anisotropy associated with big earthquakes, but they did not observe any clear temporal variations in delay times or fast orientation. In addition, studies by Liu (2004) of crustal anisotropy around the San Andreas Fault near Parkfield did not show systematic temporal variations. Moreover, a systematic 
analysis of crustal anisotropy associated with the 1999 Duzce mainshock $\left(M_{w} 7.1\right)$ along the North Anatolian Fault by Peng and Ben-Zion (2004) also showed no temporal variations before, during, or after the mainshock.

One major issue with interpreting variations in observed SWS measurements is distinguishing temporal changes in anisotropy from spatial variations in anisotropy due to differing source locations (e.g. Peng and Ben-Zion, 2005). Since the propagation direction through the crust may be different for differing source locations, the wave is likely to sample different rock mass, resulting in different splitting parameters. In a complex setting, like the Marlborough and Wellington regions where the observed anisotropy may be controlled by different mechanisms, the determination of temporal variations is challenging, even if they exist. This is due to contamination associated with the variations of ray paths as a result of changes in source locations. However, clear temporal variation in stress has been observed for large earthquakes (Lucente et al., 2010; Holt et al., 2013).

\section{Delay time and $V_{p} / V s$ ratios}

We systematically searched for temporal variations in delay time and $V_{p} / V_{s}$ associated with the 2013 Cook Strait and 2016 Kaikōura earthquakes. We applied the moving average technique described in Section 3.3.2 because our $\delta t$ and $V_{p} / V s$ measurements exhibit large scatter without any clear trends. We tried a 10-, 20-, and 30- day moving window, and all showed similar results. A time series plot of $\delta t$ and $V_{p} / V s$ measurements revealed apparent temporal variation at two stations (CAW and KIW, see supplementary Figure A.7 A and B). We stacked measurements from all 36 stations to enhance this variation observed in the individual stations (Figure 3.8A). To test whether the apparent variations originate from changes in the medium properties over time rather than changes in the source location or the propagation path, we selected a cluster of measurements within $20 \mathrm{~km}$ radius of station CMWZ (which is located in a region with a good number of measurements before and after both the 2013 and 2016 earthquakes sequence (Figure 3.8D). The apparent temporal variation of $\delta t$ and $V_{p} / V_{s}$ observed at individual stations with measurements from different locations was 

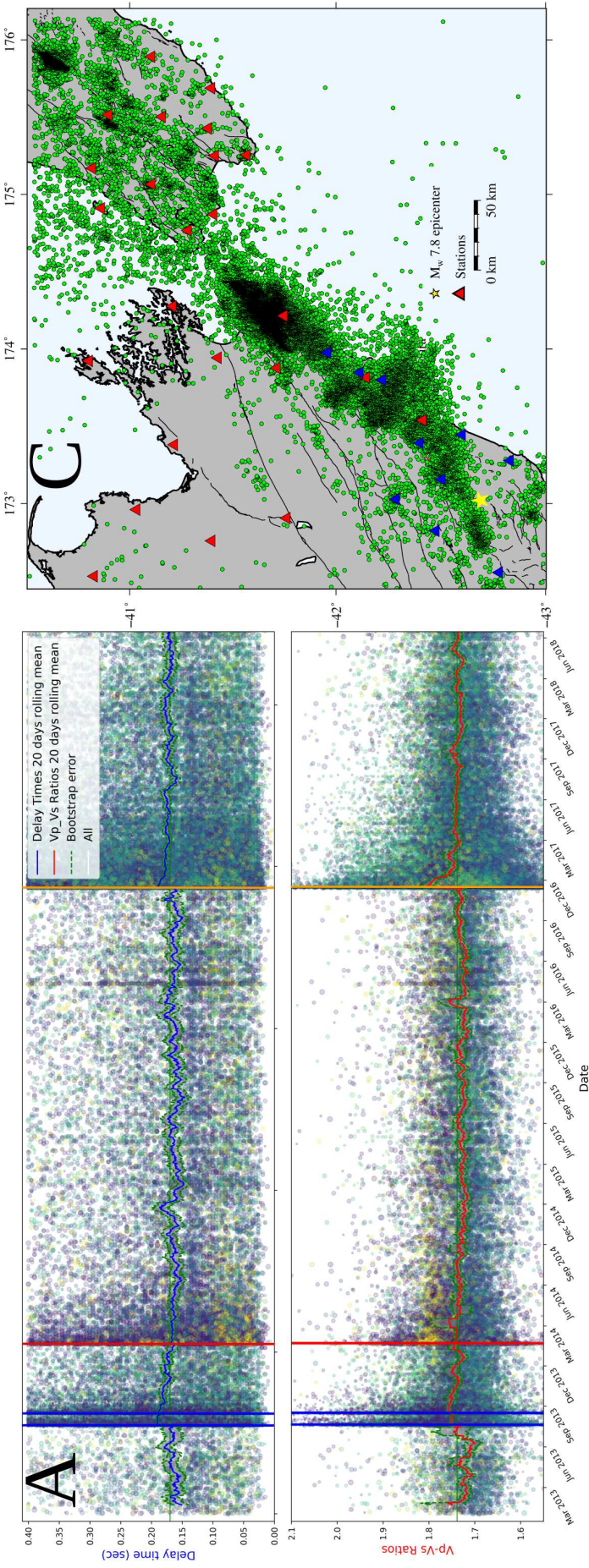
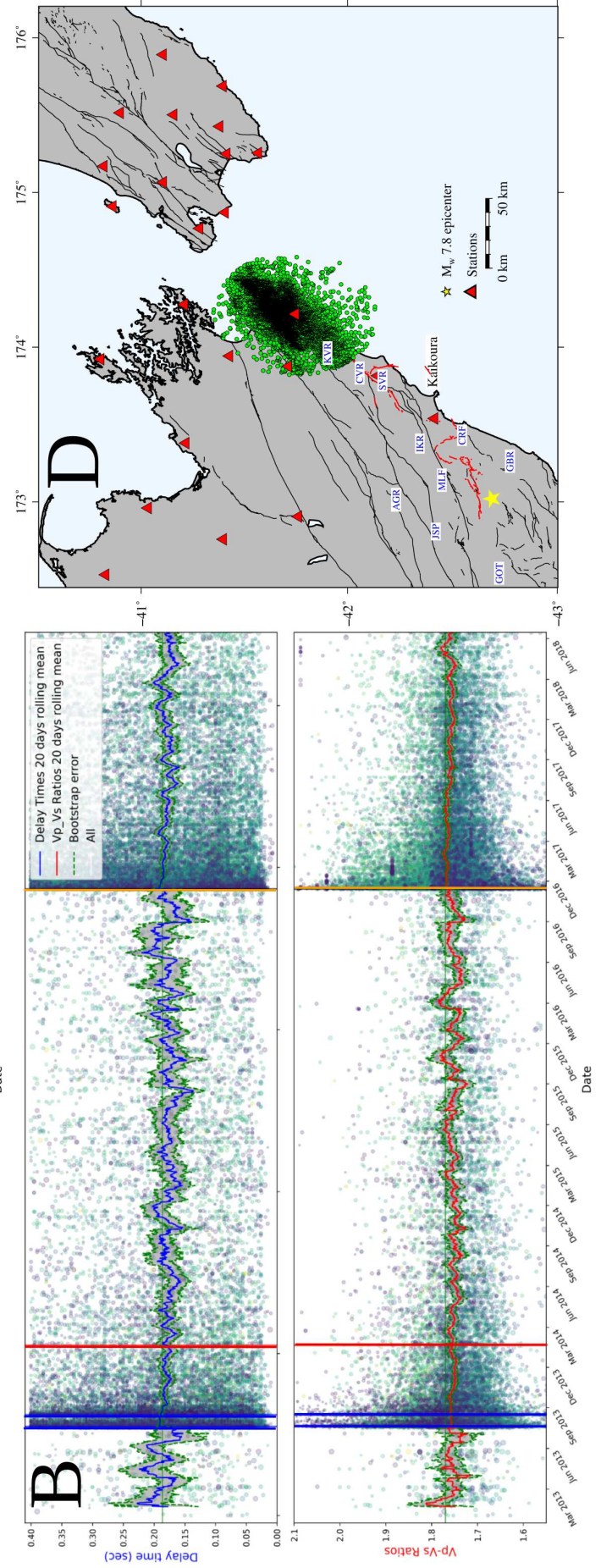

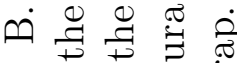

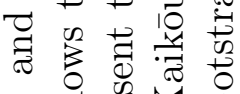
यद कर త

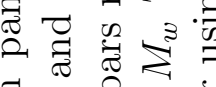
$\exists 0.0$

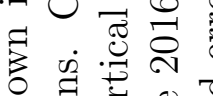
क

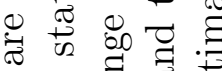
芯

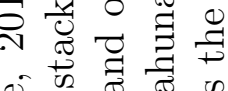

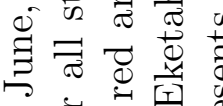
\&

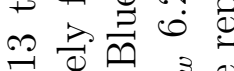
穴记

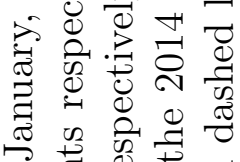
घ: $\begin{gathered}0 \\ 0\end{gathered}$

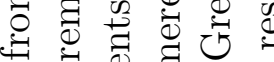
Ð

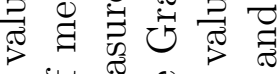

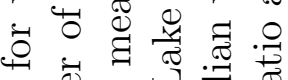

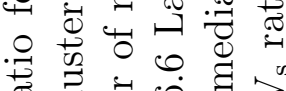

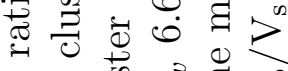
$p^{n}=$ $>^{n}$ 光

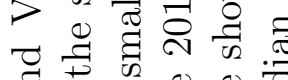
चี 范 $\ddagger$ की

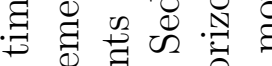
灾 壱 总 फै 岂

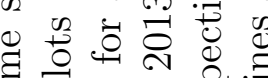

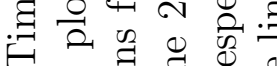
$\therefore$. $\ddot{\infty}$ 西范 ஸ口 0.0

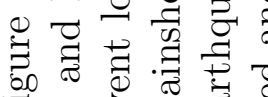
总 
not seen when the temporal analysis was done using only the small cluster of measurements (Figure A.7 $\mathrm{A}^{1}$ and $\mathrm{B}^{1}$ ). Similarly, the clear temporal variation observed (around the the $2013 M_{w} 6.52013$ Seddon, the $2013 M_{w}$ 6.6 Lake Grassmere, the $2014 M_{w} 6.2$ Eketahuna and the $2016 M_{w} 7.8$ Kaikōura earthquake) with the stacked measurements from different locations disappears when we stack individual stations measurements from earthquakes with similar locations (Figure 3.8B). Moreover, analysis of measurements with similar back azimuths revealed no clear temporal variation. This a likely indication that spatial variations of parameters strongly affect the observed apparent temporal variations.

\section{Fast orientations}

To reveal the state of anisotropy over the $5 \frac{1}{2}$ year period, we performed the spatial averaging analysis described in Section 3.3.2 with the same parameters for measurements within each year. As shown in Figure 3.9, there are significant spatial variations in the fast orientations but no systematic temporal variations. The general consistent spatial variations over the years demonstrate that neither the 2013 Cook Strait nor the 2016 Kaikōura earthquakes changed the stress directions significantly. Instead, we observe a consistent NE-SW trend (see yellow bars on Figure 3.9) that strikes sub-parallel to the active faulting around the study area. One remarkable feature is the consistent NW-SE orientation of fast orientation (blue bars) around Kaikoura region. This indicates that the NW-SE feature did not stem from the 2016 Kaikōura earthquake sequence.

The absence of temporal variations in $\phi$ suggest that any change in the stress field around central New Zealand produced anisotropy changes that are small compared to structural anisotropy. Iidaka and Obara (2013) and Nakata and Snieder (2012) reported similar observation with the M9.0 Tohoku-Oki megathrust earthquake. Iidaka and Obara (2013)'s analysis of before and after fast polarization orientations revealed no changes and they suggested that the orientation of the maximum stress axis in the region of increased seismicity did not change after the 2011 Tohoku earthquake (Iidaka and Obara, 2013). In the near-surface (i.e., upper few hundred meters) Nakata and Snieder (2012), using seismic interferometry technique, reported on no significant 

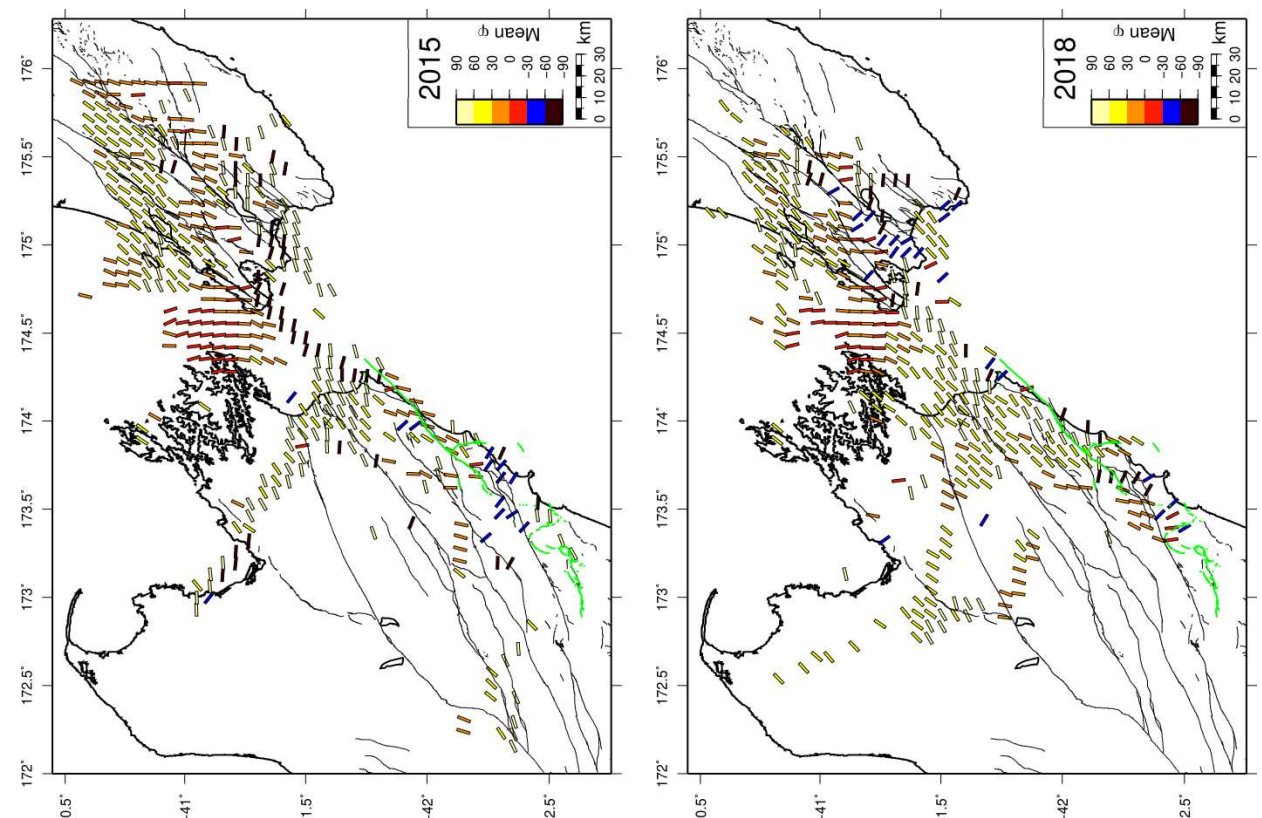

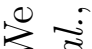
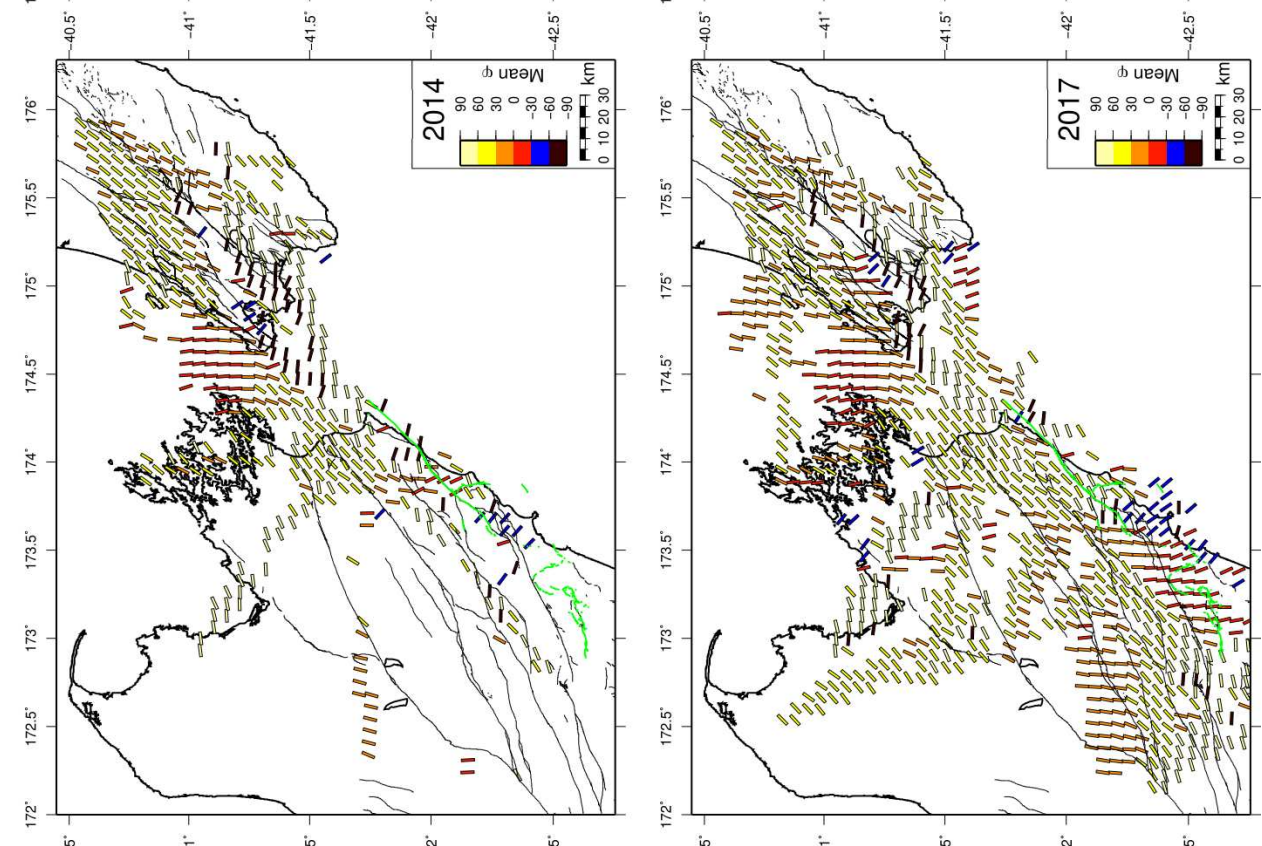

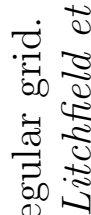

$\stackrel{-10}{0}$

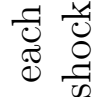

$\frac{g}{\$}$.

$+$

రृ

. సี

는

궁

蛋

.

莺

80

तथ

๘ี

ఫ串

疍

芯肯

ฮี

E

.

ठี

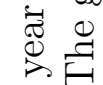
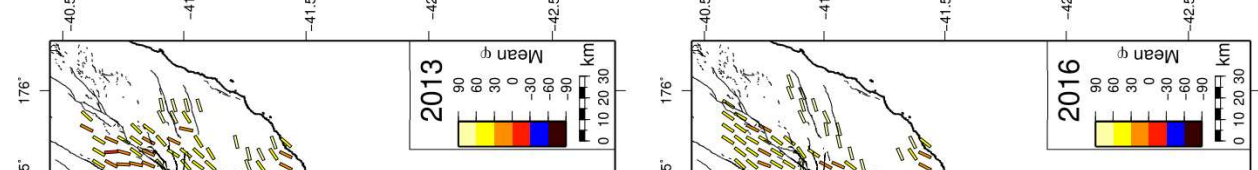

II 12

क्षें

ชี

०

家园

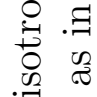

ส

㟧.

$\stackrel{0}{\circ}$

茎

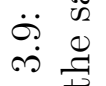

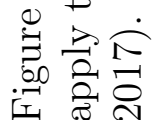


variations in fast polarization orientation after the M9.0 Tohoku-Oki earthquake. Focal mechanism inversion studies around central New Zealand following the 2016 Kaikōura earthquake (Okada et al., 2019, and personal communication with Tomomi Okada) also revealed no significant variation in the stress field. This corroborates our findings that the 2016 Kaikōura earthquake did not significantly alter the stress field around central New Zealand.

\subsubsection{Depth extent of anisotropy}

Several studies have attempted to constrain the depth extent of anisotropy. Delay times observed by Audoine et al. (2000) showed no correlation with depth, which they attributed to the higher frequency phases used in their studies, thus sampling mainly the upper crustal anisotropy. In the Marlborough region, Balfour et al. (2005) also did not observe any systematic association between $\delta t$ and depth and also suggested that the source of anisotropy may reside in the upper crust. Most of these studies conclude that anisotropy is confined to the upper few kilometers of the crust because their analysis often shows no clear correlation between delay time and hypocentral depth (Shih and Meyer, 1990; Audoine et al., 2000; Balfour et al., 2005, 2012; Gledhill, 1991).

We analyzed the association between delay time and hypocentral depth and epicentral distance at individual stations (Figure 3.10 and supplementary Figure A.8 respectively). The correlation coefficients at the analyzed stations were less than 0.2 , indicating a lack of clear correlation between delay time and hypocentral depth or epicentral distance. This suggests that we are mainly measuring anisotropy that resides only in the uppermost few kilometers of the crust (Shih and Meyer, 1990; Audoine et al., 2000; Balfour et al., 2005). Our results are also consistent with previous studies in this region and other tectonic regions (Gledhill, 1991; Audoine et al., 2000; Balfour et al., 2005; Zhang et al., 2007). The shallow sources of anisotropy observed may also suggest that topographic stresses (due to the difference in density between rock and air in the high topography areas) can contribute to the observed anisotropic pattern. 


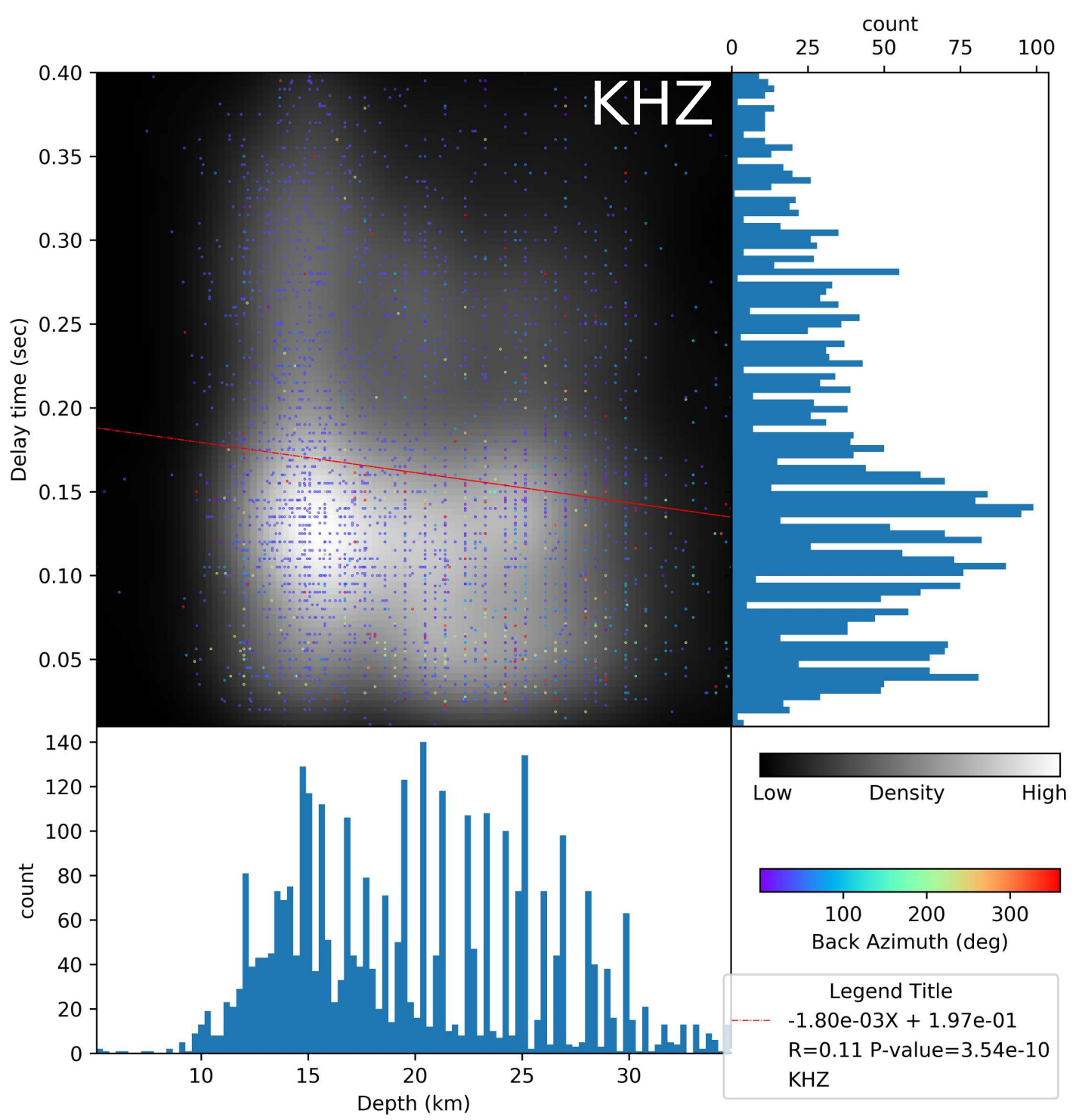

Figure 3.10: Delay times versus depth for station KHZ. Distribution of the delay time and depths are plotted on the right and bottom respectively. The scattered points are coloured by the back azimuth and plotted on a basemap of the kernel density (effectively heatmap). High-density regions are shaded white with low regions black. The red lines are the least square regression line with the equation and p-value displayed on the lower right corner of each plot.

\subsubsection{Discriminating between stress-induced and structural- control seismic anisotropy}

Discriminating between stress-induced and structural-control seismic anisotropy in most tectonic settings is often challenging because $S_{H \max }$ is often sub-parallel to major structural features (Savage et al., 1989; Peng and Ben-Zion, 2004; Johnson et al., 2011). 
But in places where the $S_{H \max }$ orientation is at a high angle to the orientation of the major structural features (such as around the eastern end of the MFS near the Kaikōura region), it is possible to distinguish between stress-induced and structurally controlled anisotropy (Boness and Zoback, 2006; Zinke, 2000). Previous crustal anisotropy studies in central New Zealand have suggested that anisotropy in the region is controlled by a combination of both structures and stresses (e.g. Audoine et al., 2000; Balfour et al., 2005; Karalliyadda and Savage, 2013; Evanzia et al., 2017). Here we attempt to distinguish between stress-induced seismic anisotropy and structurally controlled seismic anisotropy. We quantitatively compared our spatially averaged $\phi$ measurements to the average orientation of the closest active fault segments following the method described in Section 3.3.2. Figure 3.11 indicates that our spatially averaged $\phi$ measurements, $\phi_{a v g}$, are overall oriented similar to the NE-SW strike of the active fault orientation. Out of 2297 measurements we compared, $70 \%$ of them had a test statistic value, $F$, above 0.8 with only $3 \%$ less than 0.2 . The mean and median values of $F$ were $0.81 \pm 0.009$ and 0.92 respectively (thus a mean and median difference of $35^{\circ}$ and $23^{\circ}$ respectively) indicating a strong agreement. The general agreement of average $\phi$ measurements with the orientation of active fault in polygon 1 (we exclude measurements in polygon 3 whose borders are contained within polygon 1) suggests that the observed anisotropy in polygon 1 may be controlled by structures. Around the eastern end of the MFS and part of the southern end of the North Island (polygon 2 and polygon 3 on Figure 3.11) the azimuth of $\phi_{\text {avg }}$ departs from the NE-SW trend to an almost E-W trend. This departure shows a strong disagreement with the orientation of the active faults (red colour on Figure 3.11). This suggests that the anisotropy pattern observed within polygon 2 and polygon 3 is not structurally controlled. Agreement of the average $\phi$ with the orientation of active faults has also been reported elsewhere in areas of active transpression (Savage, 1999). However, structural trends are also parallel to topographic trends. In the next section, we examine how both tectonic stresses (from focal mechanism inversion) and gravitational stresses may explain part of the observed anisotropic pattern. 

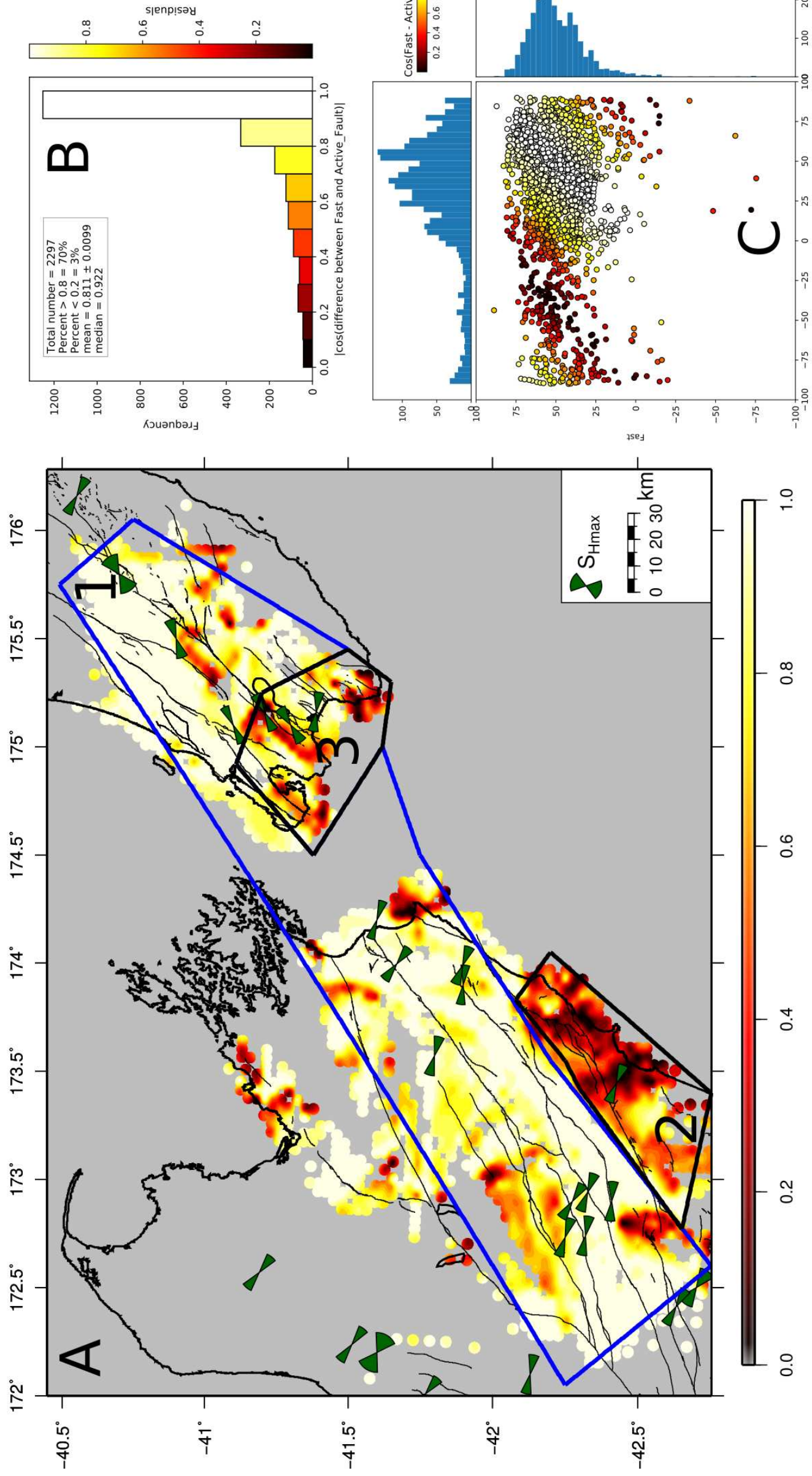

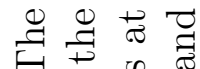

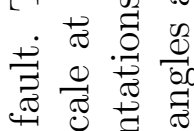

:

苋苑

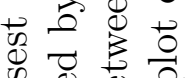

O

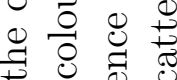

पै की

웅

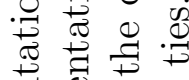

$\stackrel{0}{=}$

0 o ?

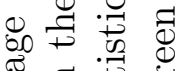

वृ क्षे

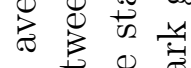

चี

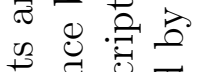

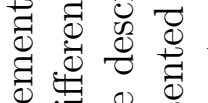

¿્વ

娄

㟧

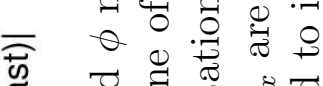

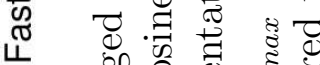

윰

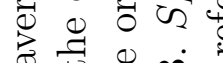

ڤ

$\exists \circ \exists . \exists$

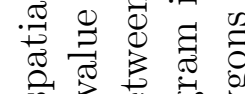

क 1000

बี

$\begin{array}{llll}0 & 0 & 0 & 0 \\ 0 & 0 & 0 \\ 0 & 0 & 0 & 0 \\ 0 & 0 & 0\end{array}$

워

要

范

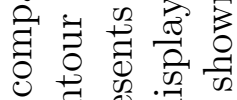

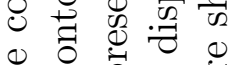

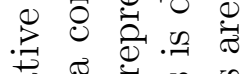

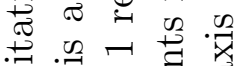

.

寻芯

$\therefore$ 渮

프류

क

$\circlearrowleft$ क⿺辶一兀

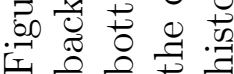




\subsubsection{Comparing spatially averaged fast directions with stress indicators}

The gravitational forces exerted by dense material placed next to less dense material, such as high mountains next to air, can significantly contribute to the total stress field, which can result in variations in crustal differential stresses (Flesch et al., 2001). This induced stress is referred to as gravitationally induced stress, $S_{H_{\max }}^{G r a v}$ (Flesch et al., 2001; Hirschberg et al., 2018). The orientation and magnitude of gravitationally induced stresses can be modelled with an assumed crustal rock density, crustal thickness and surface topography. We used the Flesch et al. (2001) method implemented by Hirschberg et al. (2018) using finite difference methods to calculate the orientation and magnitude of gravitationally induced stresses. The implementation of the Flesch et al. (2001) method involves solving force balance equations, assuming an isotropic viscous medium. Following Hirschberg et al. (2018), we assume that the viscous medium is subjected to stresses and also assume zero deviatoric stresses for the horizontal boundary condition. For our calculations, we used the $250 \mathrm{~m}$ gridded bathymetric and topographic dataset (Mitchell et al., 2012) around central New Zealand and assumed a crustal thickness and density of $30 \mathrm{~km}$ and $2,850 \mathrm{~kg} / \mathrm{m}^{3}$, respectively. We modelled the orientation and magnitude of gravitationally induced stresses at a $30 \mathrm{~km}$ grid point spacing. At every grid point, we compared averaged $\phi$ to the orientation of the gravitationally induced stresses (Figure 3.12) following the method in Section 3.3.2.

Overall, we observe a general agreement between $\phi_{\text {avg }}$ and $S_{H_{\max }}^{G r a v}$, with the mean and median values of $F$ of $0.77 \pm 0.062$ and 0.828 respectively (a mean and median difference of $39^{\circ}$ and $34^{\circ}$ respectively) (Figure 3.12B). Around the Marlborough region (Figure 3.12 ) the estimated $S_{H_{m a x}}^{G r a v}$ is parallel to the $\phi_{\text {avg }}$ orientation. The NE-SW trend of $S_{H_{\max }}^{G r a v}$ is also in agreement with the trend of the structures. Combined with the results in Section 3.5.4, this suggests the anisotropy pattern observed in polygon 1 could be controlled by either gravitational stresses or structure or a combination of the two. In polygon 2 , where agreement between the $\phi_{\text {avg }}$ and the orientation of the active fault is poor (Figure 3.11), we observe a general agreement between $\phi_{\text {avg }}$ and $S_{H_{\max }}^{G r a v}$ 


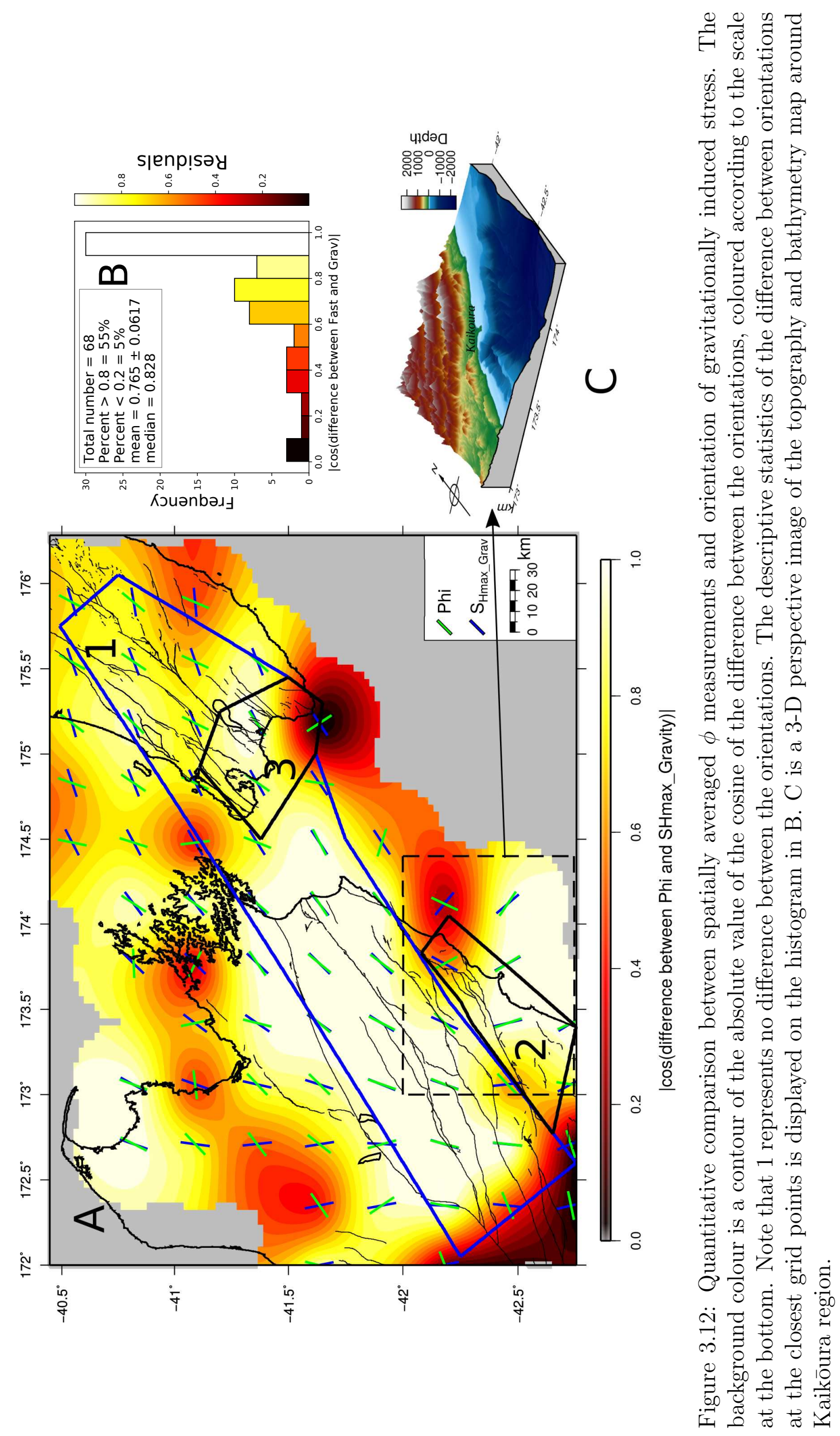


(Figure 3.12). We infer that the observed seismic anisotropy in polygon 2 (Figures 3.11 and 3.12) is best explained by the gravitationally induced stress that originates from the variations in topography around the region (Figure 3.12C).

We also examine how tectonic stresses explain the observed anisotropic patterns by comparing $\phi$ orientation measurements to the orientation of $S_{H \max }$ from focal mechanisms. Figure A.9, in the supplementary material, illustrates the comparison of $\phi$ orientations with the nearest $S_{H \max }$ following the quantitative analysis described in Section 3.3.2. Around the northern South Island, we observe a general disagreement between $\phi_{\text {avg }}$ and $S_{H \max }$ orientations (mean $F$ of $0.47 \pm 0.01,62^{\circ}$ angle difference; see supplementary Figure A.9). The general disagreement observed in the South Island is an indication that a different source of anisotropy, other than tectonic stresses is needed to explain the anisotropic pattern. Since the mean depth distribution of the focal mechanism inversion used for comparison is about $16.12 \pm 2.08 \mathrm{~km}$ (see supplementary Figure A.9 C), we suggest that the tectonic stresses are acting on deeper structures than the measured anisotropic structure. Our results reinforce Balfour et al. (2005)'s observations that the tectonic stress field does not control the crustal anisotropy pattern around the Marlborough region. Conversely, in the North Island, the $\phi_{\text {avg }}$ orientations generally agree with $S_{H \max }$ orientations (mean $F$ of $0.75 \pm 0.02,42^{\circ}$ angle difference). This agreement is an indication that tectonic stresses may contribute to the observed anisotropic pattern, although as shown in Section 3.5.4, structure may also explain the anisotropy here. Evanzia et al. (2017) observed a similar agreement and suggested that stresses in the overriding plate and gravitational stresses control the anisotropic pattern in the Wellington region.

\subsubsection{Comparing the geodetically determined plane of maxi- mum shear stress with spatially averaged fast directions}

In the Marlborough and Wellington regions the tectonics are complicated by the locked part of the Hikurangi subduction zone (Williams et al., 2013) and a series of parallel strike-slip faults (MFS). In Figure 3.13, spatial averaging of $\phi$ orientations is compared 
with the plane of maximum shear stress (we assume to be $45^{\circ}$ to the principal axes, compressional and extensional plane, of the strain rate field (Ciucu, 2010)). We used the strain rate field result calculated by Lamb et al. (2018). The observed NE-SW trend of the $\phi$ orientation, which generally shows good agreement with the plane of maximum shear stress (polygon 1 on Figure 3.13), probably reflects the effect of the shear deformation along the Australian/Pacific boundary.

Present deformation along the plate-boundary zone in north eastern South Island, New Zealand, is expected to influence anisotropy (Wilson et al., 2004; Balfour et al., 2005; Karalliyadda and Savage, 2013; Chen et al., 2013). Okaya et al. (2016) suggested that the subhorizontal foliations with lineations (e.g. schist fabric), developed due to pervasive shearing in strike-slip fault zones, are a possible source of anisotropy. We, therefore, infer that the margin-parallel orientation of $\phi$ observed could be caused either by gravitational stress or shear deformation across the Marlborough region as a result of shearing along the active faulting (MFS) or both. The general disagreement of the orientations observed in polygon 2 underpins our earlier interpretation (Section 3.5.4) that these regions are likely controlled by gravitational stresses.

\subsubsection{What controls seismic anisotropy in central New Zealand}

Several studies around the MFS and the Wellington region (e.g. Gledhill, 1991; Audoine et al., 2000; Matcham et al., 2000; Balfour et al., 2005; Karalliyadda and Savage, 2013; Evanzia et al., 2017) suggest that the anisotropy in the crust is either structuralcontrol or stress-induced. Here, we try to delineate regions where the proposed source of anisotropy best explains our results by comparing the mean values on the $F$ statistic in each polygon, both North and South Island, and the entire study region. We looked at how the structural-control hypothesis (using active fault and maximum shear plane orientations as a proxy) and stress-induced hypothesis (relating to gravitational and tectonic stresses) explain the observed anisotropic pattern. In polygon 1 (Figure 3.14, and for regions see Figure 3.11), both gravitational stresses and structures can explain (with the mean $F>0.8$ ) the anisotropic pattern observed. This is because the active faults and foliation, or aligned minerals (due to shearing) in the area are oriented paral- 


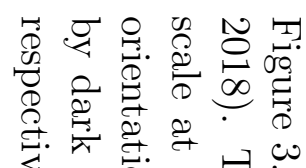

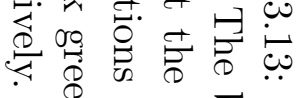

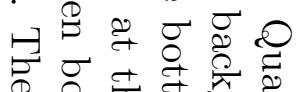

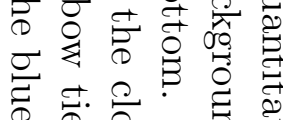

क क्ष

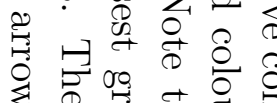

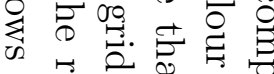

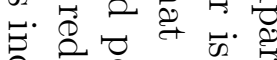

2. 0.

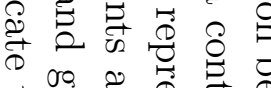

声贻

물 蚂

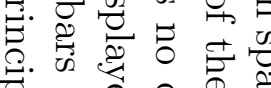

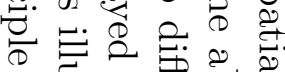

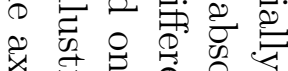

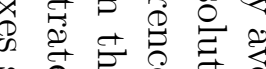

ये ते के के

స्.

ज

๑े

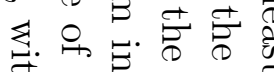

范

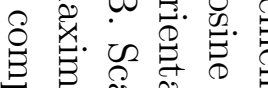

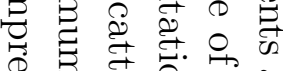

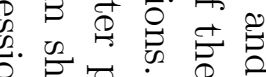

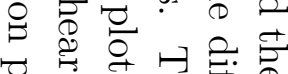

ㅇ. 웅

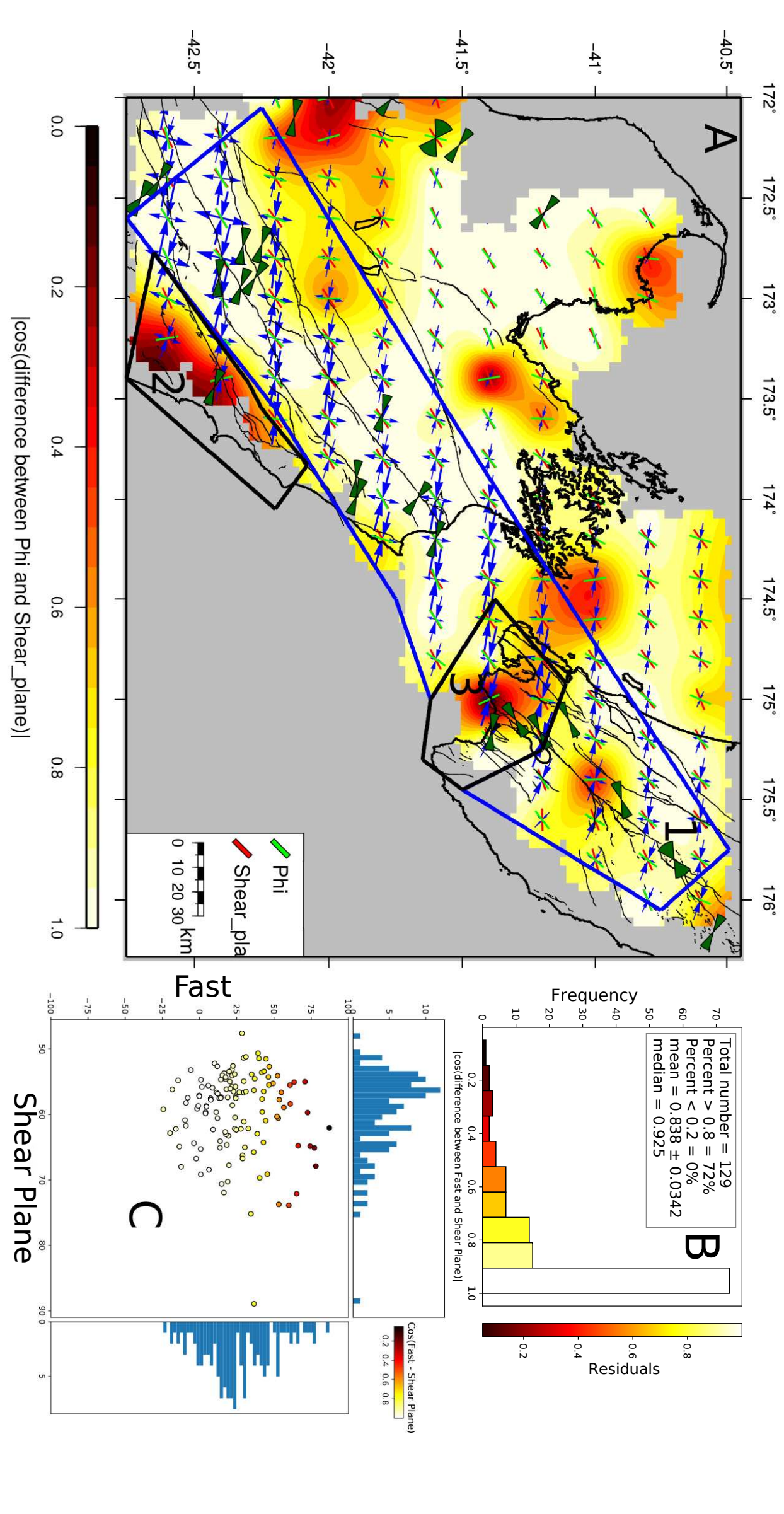

节蛋

․ :

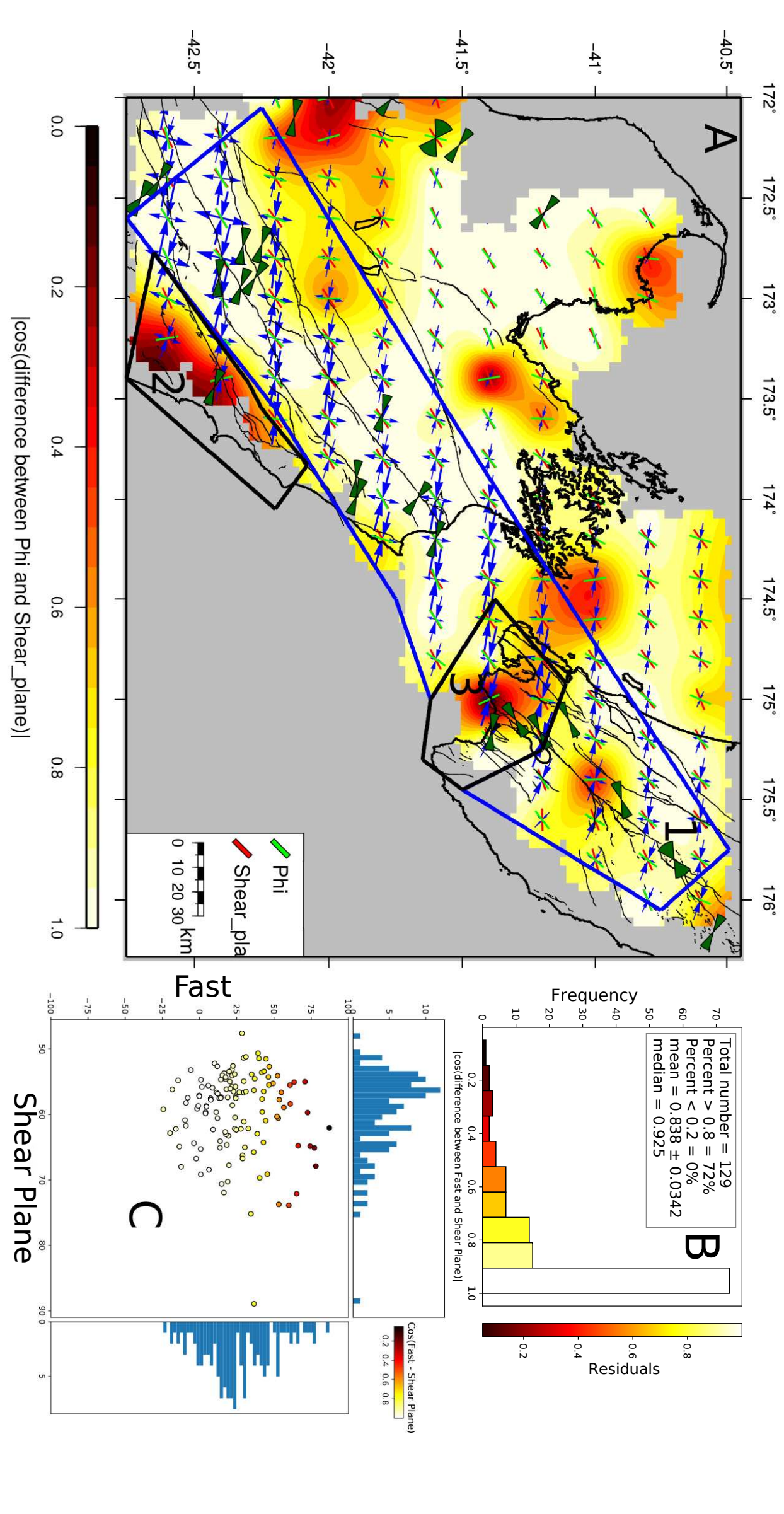

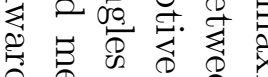

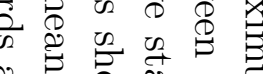

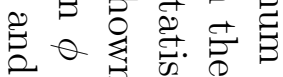

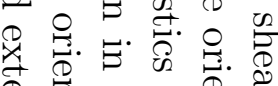

孛 $\Omega$ 号

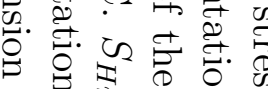

き

¿

के 0 \& 0

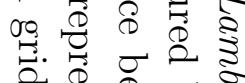

प)

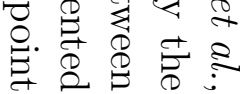


lel to the strike of the topographic structures, which are also the ridges. Gravitational stresses are the hypothesis that best explains the anisotropic pattern in polygon 2 (with the mean $F>0.8$ ). This is likely due to the sharp topography contrast (Figure 3.12C) observed around the Kaikōura region. In polygon 3, no single hypothesis can explain the observation (with the mean $F<0.7$ ). In the North Island, overall either stresses (tectonic and gravitational) or structures or both explain our results. However, in the South Island, gravitational stresses and structures best explain our results. Overall the gravitational stress-induced hypothesis can explain most regions. However, structural controls cannot be ruled out for any region except polygon 2 .

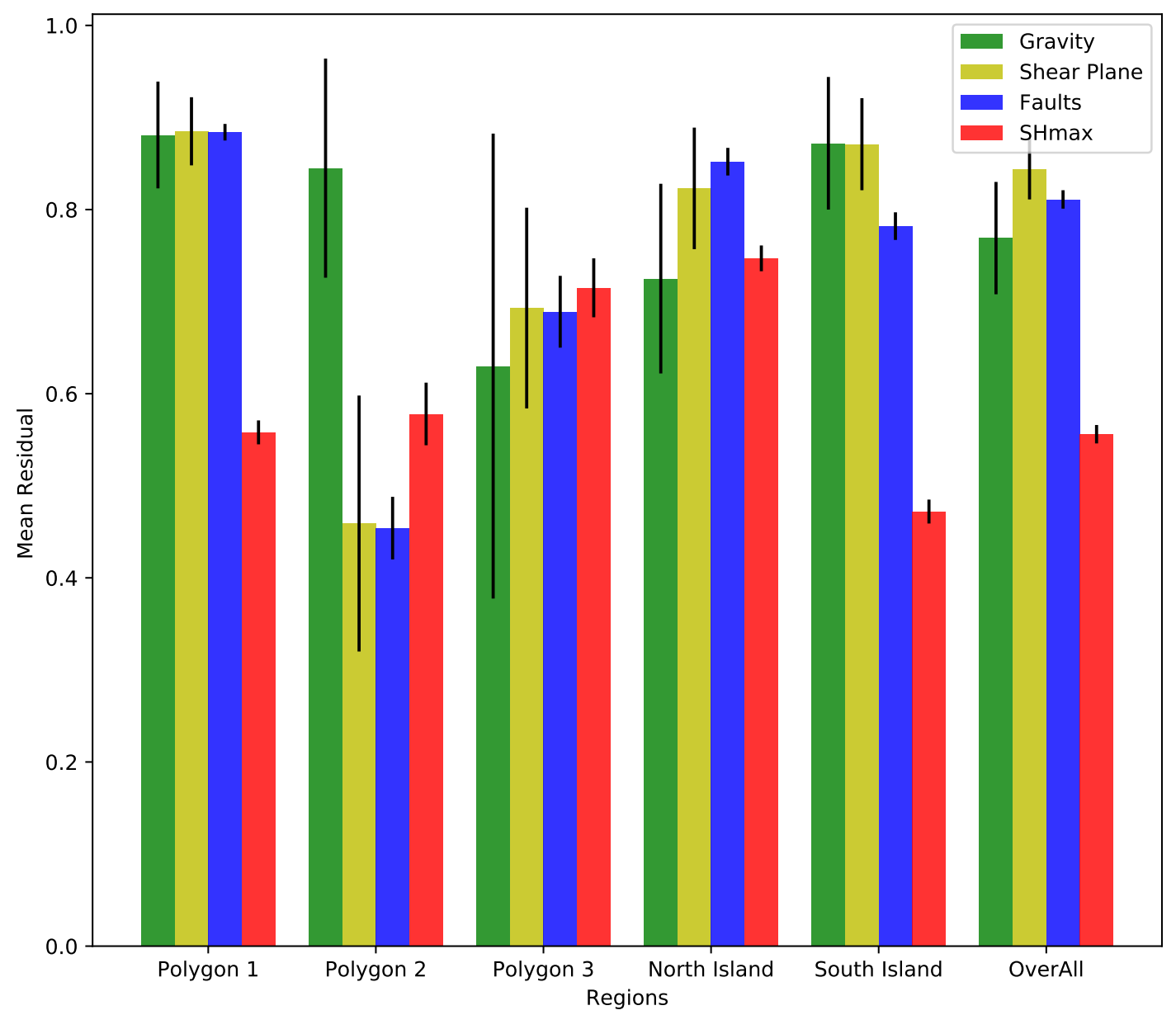

Figure 3.14: Qualitative comparison of the possible source of crustal anisotropy around the study area. The bar chart is grouped by the delineated regions and in each region, the proposed sources of crustal anisotropy (colour corded in the legend) are compared. Green, blue, red and yellow bars represent gravitational stresses, active fault orientation, $S_{H \max }$ orientation from the focal mechanism and maximum shear plane orientation, respectively. The thick vertical lines on each bar represent the mean error of the residuals. 


\subsection{Conclusions}

We have presented the largest number of measurements of high-quality SWS parameters $(\sim 102,000)$ around the Marlborough and Wellington region with a detailed systematic analysis of crustal anisotropy using over 40,000 crustal earthquakes recorded on at least one of the 36 stations. The size of our data set has allowed us to show the detailed spatial and temporal variation of anisotropic structure around central New Zealand. We conclude that the crustal anisotropy around the study region is confined to the upper few kilometers of the crust and can be controlled by either one mechanism or a combination of more than one. The high correspondence between $S_{H \max }$ calculated from gravitational potential energy from topography and average fast polarization orientation around the Marlborough and Kaikōura region suggests that gravitationally induced stresses contribute to the crustal anisotropy around the region. In the North Island, tectonic stresses inferred from focal mechanisms as well as geological structures could contribute to the observed anisotropy. In the South Island, gravitational potential energy gives the best overall match but inherited geological structures could contribute and control some regions. We suggest that examining the effect of gravitational stresses on crustal seismic anisotropy should not be neglected in future studies. We do not observe any spatiotemporal variation of the state of anisotropy over the $5 \frac{1}{2}$ year period. Apparent temporal variations of $\delta t$ and $V_{P} / V_{S}$ are mainly due to spatial variations of anisotropy combined with temporal varying paths of seismic waves. 


\section{Anisotropic Structure on a scale of tens of meters within Whataroa Valley in the vicinity of the Alpine Fault}

\section{Abstract}

Characterizing the in-situ properties and conditions of a fault approaching failure is an important step towards understanding the mechanics of earthquakes. The Alpine Fault, New Zealand provides a rare opportunity to do so. In this study we characterize the anisotropic structure and infer the likely mechanism that controls the anisotropy in the Whataroa Valley in the vicinity of the Alpine Fault, using a dense seismograph array (159 stations) with $\sim 35 \mathrm{~m}$ station separation. Due to the low seismicity in the region, we used both the energy-based (short-term average/long-term average) and matched-filter detection algorithms to detect the earthquakes, and located them using the HYPOCENTER location algorithm. A total of seven events were successfully located within the six days of sensor deployment, with local earthquake depths ranging from 1 to $8 \mathrm{~km}$. We used the automatic and objective splitting analysis code (MFAST) to estimate the splitting parameters. A total of 1090 (out of 1304) high-quality measurements were obtained from the 159 stations using the seven events. The mean orientation of the fast direction was $47 \pm 23^{\circ}$ which is near parallel to the local strike $\left(\sim 55^{\circ}\right)$ of the Alpine Fault trace and the orientations of the regional and borehole foliation planes. The fast directions observed show no significant spatial variation. The small delay time of $70 \pm 3 \mathrm{~ms}$ we observed is expected of small amount of splitting of the high-frequency phases (dominant frequencies $8.45 \pm 0.08 \mathrm{~Hz}$ ). We infer that the anisotropic structure in the Whataroa Valley is structurally controlled by the Alpine 
Fault fabric and foliations within the valley.

\subsection{Introduction}

Seismic anisotropy, a phenomenon where the velocity of an elastic wave varies as a function of the direction of propagation, is ubiquitous in the Earth's crust (Crampin and Booth, 1989; Savage, 1999; Boness and Zoback, 2006; Crampin et al., 2015; Savage et al., 2016; Cochran and Kroll, 2015). Often, the geometry and amount of seismic anisotropy are estimated using shear-wave splitting measurements. The term shear wave splitting (SWS) is used to describe the phenomenon where a shear wave propagating through an anisotropic medium splits into two orthogonal polarized waves traveling at different velocities. The orientation of the fast shear wave (fast direction) and the time lag between the fast and slow wave (delay time) are used to describe the geometry and strength of seismic anisotropy respectively. Existing studies (e.g. Crampin and Booth, 1989; Savage, 1999; Boness and Zoback, 2006; Crampin et al., 2015; Savage et al., 2016; Cochran and Kroll, 2015) have reported mechanisms such as; (a) preferred opening or closing of cracks resulting from applied stresses (stress-induced), (b) shear planes and foliation planes, fault grain (structural controll), and (c) mineral alignment (intrinsic anisotropy) as common mechanisms that control anisotropy in the crust. Recent studies also suggest that gravitational stresses (forces exerted by dense material placed next to less dense material, such as high mountains next to air) are a possible mechanism controlling anisotropy (e.g. Araragi et al., 2015; Illsley-Kemp et al., 2017, and Chapter 3 of this thesis). These studies also suggested that SWS can be used to probe the in situ properties of the upper crust. In regions of active faulting, the fast directions $(\phi)$ have been used to investigate the orientation of the maximum horizontal compressive stress $\left(S_{H \max }\right)$ and the orientation of fault strands or aligned shear fabric (Evans, 1984; Aster et al., 1990; Zinke, 2000; Boness and Zoback, 2006; Cochran and Kroll, 2015; Crampin et al., 2015; Savage et al., 2016). For stress-induced anisotropy, $\phi$ is often sub-parallel to $S_{H \max }$ (e.g. Crampin and Booth, 1989; Crampin and Gao, 2006; Savage et al., 2015) and in the case of structurally controlled anisotropy, $\phi$ is 
often reported to be near parallel to the fault or foliation orientation (e.g. Zinke, 2000; Boness and Zoback, 2006). Most of these studies have been conducted on mature, well-developed fault structures, that are often recently ruptured. However, few studies have conducted experiments to characterize the anisotropic structure on a fault late in its earthquake cycle. The New Zealand Alpine Fault offers a rare opportunity to study and document the anisotropic structure of a fault approaching failure (Sutherland, 1999; Sutherland et al., 2007; Howarth et al., 2015, 2018). Moreover, most SWS studies are conducted with station separations of the order of thousands of meters, but in this study, we use station separation on the order of tens of meters. In this Chapter, we aim to investigate the anisotropic structure, characterize spatial variability in seismic anisotropy, and infer the likely mechanism that controls the anisotropy in the Whataroa Valley in the vicinity of the Alpine Fault.

The Alpine Fault, a mature dextral strike-slip fault, connects the Hikurangi and Puysegur subduction zones with a continuous surface trace of $\sim 420 \mathrm{~km}$ in the South Island of New Zealand (Norris and Toy, 2014). At a scale of tens of kilometers, it appears as a remarkably linear feature. The Alpine Fault is thought to accommodate about $65-75 \%$ of the total Australian-Pacific relative plate boundary movement (Beavan et al., 1999; Norris and Cooper, 2001; Little et al., 2005). From detailed paleoseismicity studies, the Alpine Fault is known to produce large earthquakes every $291 \pm 23$ years and it is inferred to be late in its earthquake cycle (Sutherland, 1999; Sutherland et al., 2007; Howarth et al., 2015, 2018), posing the largest seismic hazard in the South Island (Howarth et al., 2018). To provide insight into the rock properties, the formation of plate boundaries, and mechanisms of earthquake processes, two scientific drilling experiments (Deep Fault Drilling Project, DFDP) were undertaken in and around Whataroa Valley in the vicinity of the central Alpine Fault (Townend et al., 2009). The first phase of the DFDP (DFDP-1) took place in the south of Whataroa Valley (Gaunt Creek), where two shallow (128 and $98 \mathrm{~m}$ deep) boreholes were drilled to intersect the principal slip zone of the Alpine Fault (Boulton et al., 2014). The fault zone was found to be highly fractured yet impermeable, with high geothermal gradient $\left(\sim 68^{\circ} \mathrm{C} / \mathrm{km}\right)$ and high coefficients of friction ( $\mu=0.61$ to 0.76$)$ (Sutherland et al., 2012; 
Townend et al., 2013; Boulton et al., 2014). In late 2014, the second phase of the DFDP (DFDP-2) took place in the Whataroa Valley, with the aim of drilling through the Alpine Fault at a depth of approximately 1000 m. Due to technical difficulties, a depth of $\sim 825 \mathrm{~m}$ was reached (Sutherland et al., 2017; Townend et al., 2017; Toy et al., 2017). Extreme hydrothermal conditions with measured geothermal gradient of $125 \pm 55^{\circ} \mathrm{C} / \mathrm{km}$, elevated pore fluid pressure (10\% above hydrostatic levels), and high permeability were observed beneath the Whataroa Valley (Sutherland et al., 2017; Townend et al., 2017; Toy et al., 2017). Sutherland et al. (2017) suggested that the extreme hydrothermal conditions were due to fluid flow within the fractured rocks and the exhumation of hotter rocks from depth.

In early 2016 as part of the Deep Fault Drilling Project, a detailed geophysical survey was carried out in the Whataroa Valley near the DFDP-2 drill site (Townend et al., 2018). The 2016 Whataroa Active Source Seismic Experiment was carried out to acquire a high-resolution geophysical image of the central Alpine Fault using multiple geophysical acquisition systems to better understand the complex geology and determine the velocity structure surrounding the DFDP-2 drill site. The study made use of improved acquisition techniques and geometry and included borehole sensors (which were installed in the DFDP-2 borehole), arrays of one- and three-component surface sensors, and a large number of highly repeatable vibroseis shot-points on multiple profiles (Townend et al., 2018). In this Chapter, we make use of this dense deployment and local earthquakes to characterize the anisotropic structure at a scale of tens of meters within the Whataroa Valley in the vicinity of the Alpine Fault.

\subsubsection{Previous studies}

The Whataroa Valley and its environs are some of the best-studied sites (in both seismological and geological properties) along the central Alpine Fault because: (1) the area is along an exhumation trajectory with a high uplift rate (that exposes deep rocks to the near-surface), (2) the area has a history of previous geophysical and geological experiments, and (3) the site is easily accessible (Norris and Cooper, 2001; Okaya et al., 2002; Norris et al., 2012; Norris and Toy, 2014; Little et al., 2005). In late 1995 the 
South Island Geophysical Transect, SIGHT, (a 2D seismic line across the South Island) went through the Whataroa Valley (Avendonk et al., 2004). In 1998 the extension of SIGHT, Whataroa98, was undertaken in the Whataroa Valley to image the Alpine Fault and crustal structures in the valley (Davey et al., 1998). Lay et al. (2016, 2020) recently used seismic reflection data to image the subsurface around Whataroa Valley. They observed complex subsurface structures and a high-velocity layer at the top of the basement that was attributed to anisotropy (Lay et al., 2016, 2020). Around the Whataroa Valley, Jenkins et al. (2019) combined gravity models from high-precision gravity surveys and surface mapping to constrain the location on the surface trace of the Alpine Fault. They also observed a high horizontal gravity gradient near the DFDP-2 site and suggested that an active reverse fault might bound the base of the hillslope (Jenkins et al., 2019). Upton et al. (2017) explored the relationships between topographic relief, fault partitioning, and fault strength by geodynamically modelling the motion and stress relations for a 3D deforming crust. Upton et al. (2018) summarized research conducted on the Alpine Fault from a range of disciplines including tectonics, seismology, paleoseismology, and social science as part of the Tercentenary of the 1717 AD Alpine Fault earthquake under the theme "advances in science and understanding hazards".

The stress field near the central Alpine Fault has been estimated by several researchers who have found the orientation of the maximum horizontal compressive stress, $S_{H \max }$, to be between $110-120^{\circ}$ (see green bow ties on Figure 4.1; Leitner et al., 2001; Boese, 2012; Michailos et al., 2020). The $S_{H \max }$ orientation is about $65^{\circ}$ oblique to the strike of the Alpine Fault and parallel to the azimuth of maximum contractional strain rate (which typically trends $\sim 110-120^{\circ}$ ) from GNSS data (Beavan et al., 1999). Leitner et al. (2001) analysed 53 focal mechanisms to estimate the orientation of $S_{H \max }$ and the stress regime. Their results revealed a strike-slip stress regime and $S_{H \max }$ of 110-120. Boese (2012) obtained highly consistent $S_{\text {Hmax }}$ estimates around the central Alpine Fault with an average of $115 \pm 10^{\circ}$. Recently, Michailos et al. (2020) carried out a detailed spatio-temporal analysis of the tectonic stress regime near the central Alpine Fault using 845 focal mechanisms. They observed consistent spatial and temporal char- 
acteristics of the stress parameters with an average $S_{H \max }$ orientation of $122 \pm 11^{\circ}$. In and around the Whataroa Valley the orientation of $S_{\text {Hmax }}$ is $\sim 118 \pm 26^{\circ}$ (Michailos et al., 2020).

Savage et al. (2004, 2007) used strain and mantle flow modelling to study seismic anisotropy and coupling at strike-slip boundaries to understand the origin of seismic anisotropy and to constrain teleseismic shear wave splitting interpretations around the Alpine Fault. Karalliyadda and Savage (2013) and Zietlow et al. (2014) used shear wave splitting to study the regional anisotropy in the South Island using both local and teleseismic events. Okaya et al. (1995) investigated anisotropy of the Haast Schist near the Alpine Fault fault zone and observed a high percentage $(\sim 17.3 \%)$ of anisotropy. They reported that the faster wave, which is parallel to foliations in the region, and the slower wave, show velocity differences up to $1000 \mathrm{~m} / \mathrm{s}$. They further suggested that seismic anisotropy around the Alpine Fault fault zone is caused by preferred mineral orientation and is not likely due to the presence of cracks (Okaya et al., 1995). Surprisingly, Pulford et al. (2003), using active source data, observed small delay times (with a maximum of $0.08 \mathrm{~s}$ ) around the most deformed part of the Alpine Fault region. They attributed these small delay times to the high-frequency waveforms used for estimating their shear wave splitting parameters (Pulford et al., 2003). To our knowledge, there have not been any published crustal anisotropic studies in and around the Whataroa Valley, hence the need to characterize the anisotropy structure near this fault as it approaches failure.

Several laboratory and experimental seismic anisotropy studies of rocks sampled from the Alpine Fault and it environs (e.g. Okaya et al., 1995; Dempsey et al., 2011; Simpson et al., 2020; Adam et al., 2020) have reported that anisotropy is much stronger in and around the Whataroa Valley (than it surrounding) which they infere to be due to the presence of mylonites and the schists in and around the valley. Dempsey et al. (2011) used electron backscatter diffraction and field measurement of muscovite, mylonites, and biotite (mica rich minerals) to examine the seismic velocity anisotropy of the Alpine Fault Zone. They observed that the fast direction for both P- and S- wave are strongly dependent on the foliations of the mica (indicative of mineral 
controlled anisotropy). They also reported a P- and S- wave anisotropies $12 \%$ and $10 \%$ respectively.

Around and within the Whataroa Valley, fracture imaging and analyses from both outcrops and borehole samples of the hanging-wall of the Alpine Fault wall revealed a dominant NE-SW orientation (Williams et al., 2017; Massiot et al., 2018). Massiot et al. (2018) suggested that within the Whataroa Valley, the orientation foliation is likely sub-parallel or similar to the plane of Alpine Fault at depth of about $1 \mathrm{~km}$.

\subsubsection{Tectonics and seismicity around the Whataroa Valley}

The Whataroa Valley is a former glacial valley filled with unconsolidated glacial and river sediments and is located in central Westland, South Island, New Zealand (Figure 4.1). Around the valley, the Alpine Fault strikes at approximately $055^{\circ}$ as an oblique thrust fault (Little et al., 2005; Cox et al., 2008; Barth et al., 2012; Langridge et al., 2018). The orientation of the foliations on average is consistently (over many kilometers) parallel to the strike of the Alpine Fault, although the presence of mylonite or schist may cause local departures (see black thick lines on Figure 4.1; Little et al., 2005; Litchfield et al., 2013). Gillam et al. (2013) found the mean orientation of the foliation of outcrops in the Tatare Stream (north east of the Whataroa Valley) to strike at $053^{\circ}$ with a dip of $63^{\circ}$. For outcrops in the Whataroa Valley, Little et al. (2002) measured a mean foliation of $55^{\circ}$ (strike) and $50^{\circ}$ dip. The orientation of fractures and foliation planes identified from the acoustic borehole televiewer (BHTV) in the DFDP-2 borehole are $57^{\circ}$ and $53^{\circ}$ respectively (Townend et al., 2017; Massiot et al., 2018). Geomorphology and shallow structural studies within the Whataroa Valley show a change from strike-slip faults to oblique thrusts in the valley (Norris et al., 2012; Norris and Toy, 2014). From airborne Light Detection and Ranging (LiDAR) data, a scale-dependent partitioning (unpartitioned, serially partitioned, and parallel partitioned) along the fault has been observed (Barth et al., 2012; Langridge et al., 2014). Langridge et al. (2018) recently tried to determine the poorly located active traces of the Alpine Fault near the Whataroa Valley using high-resolution topographic data derived from airborne LiDAR. They identified a $300 \mathrm{~m}$ wide zone of partitioned 
faulting close to the DFDP-2 drill site (Langridge et al., 2018).

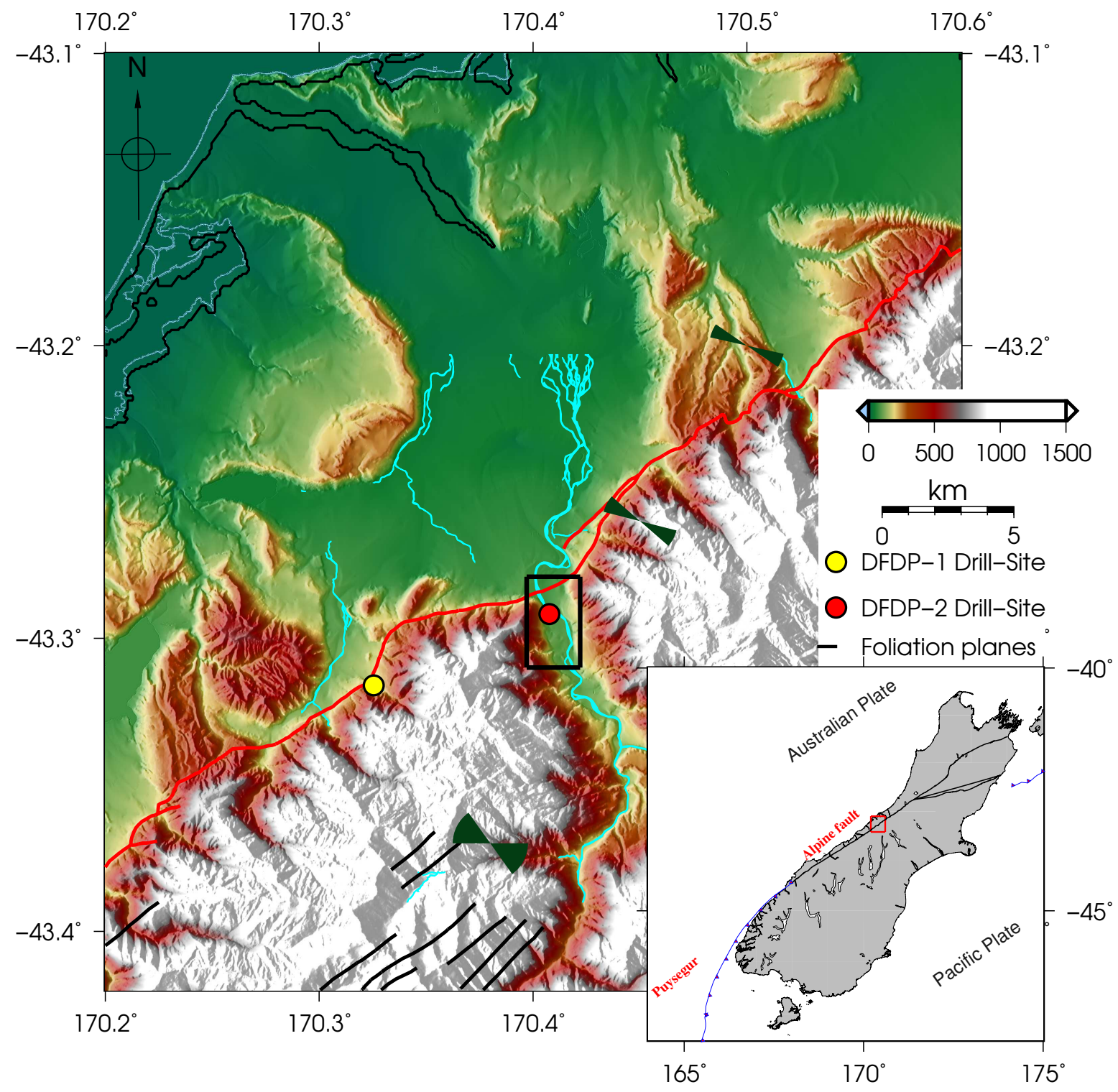

Figure 4.1: Overview map of the study area and its environs showing the position of the DFDP-1 and DFDP-2 drill sites (yellow and red circles respectively), mapped fault trace (red; Litchfield et al., 2013), Whataroa river (cyan line passing through the black box), foliation planes from the GNS QMAP database (black lines) and Little et al. (2002). Average regional $S_{H \max }$ orientations from Leitner et al. (2001) (bottom left), Boese (2012) (top right) and Michailos et al. (2020) (middle) are represented by dark green bow ties with wedges showing 95\% confidence intervals. The location of the 2016 Whataroa Active Source Seismic Experiment (WASSE) is marked by a black rectangle. See Figure 4.2 for more details of the WASSE survey. The inset shows the South Island of New Zealand with the location of the main map (red box).

The Whataroa Valley is characterized by anomalously low levels of seismic activity as documented in different studies (Leitner et al., 2001; O'Keefe, 2008; Boese, 2012; Bourguignon et al., 2015; Chamberlain et al., 2017a; Michailos et al., 2018). The surroundings of the Whataroa Valley are zones of relatively higher rates of microseismity 


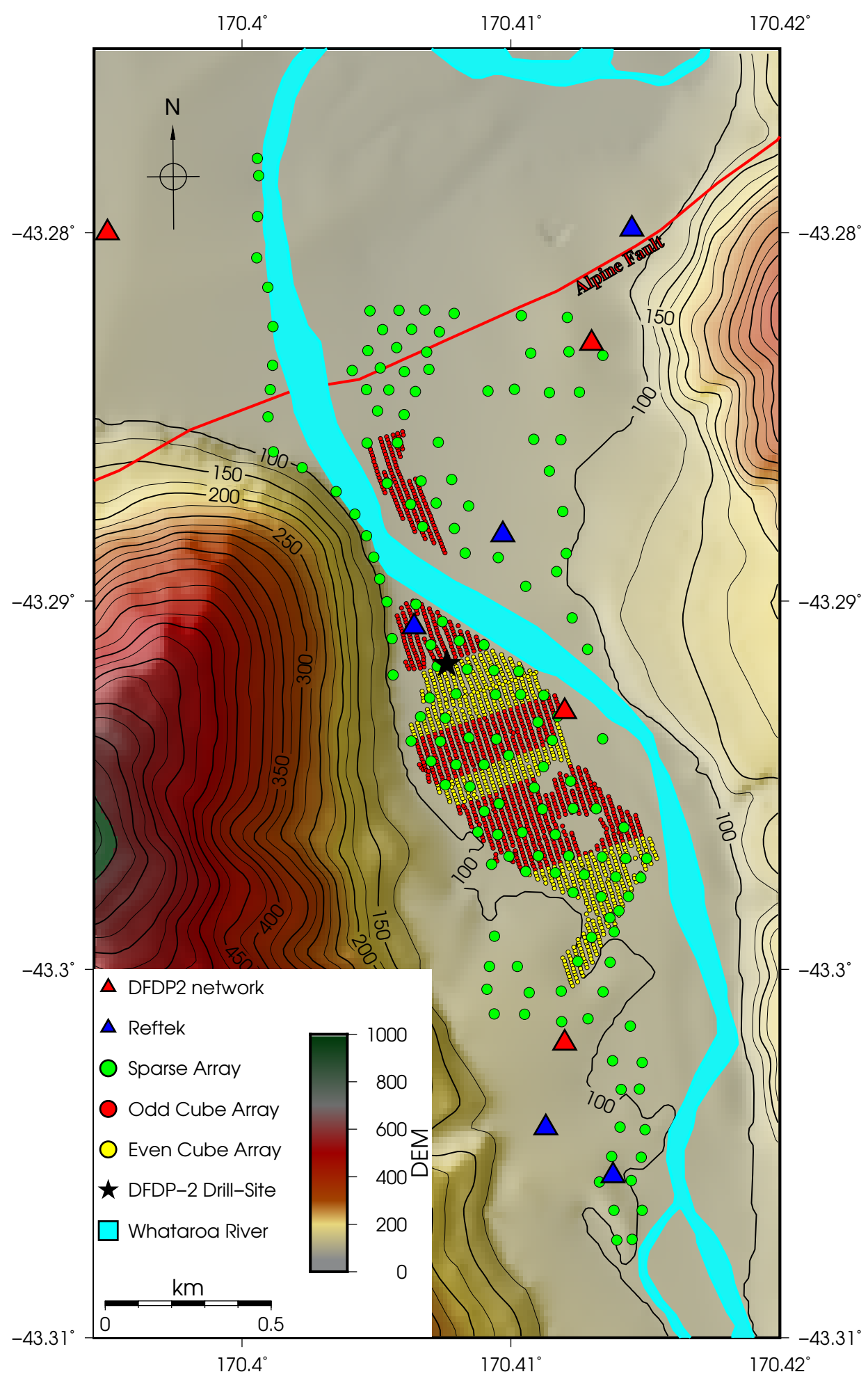

Figure 4.2: Map showing the seismic stations used for analysis. Green circles show the 159, 3C seismometers surrounding the Deep Fault Drilling Project (DFDP-2) site with an average spacing of $35 \mathrm{~m}$. The yellow and red circles show the even and odd patch array deployed in a rolling mode with a spacing of $10 \mathrm{~m}$ perpendicular and $20 \mathrm{~m}$ parallel to the main strike of the Alpine Fault. Each patch operated during a different time. The red and blue triangles are the DFDP-2 network and Reftek stations respectively, used for event detection and location. For tidy presentation, station WHAT2 (which is part of the stations used for location) is plotted on Figure 4.4 instead. 
with magnitudes (M 0-4) (Boese, 2012; Bourguignon et al., 2015). The area of low seismicity is surrounded by fewer mapped faults (Cox et al., 2008). Several researchers have tried to explain the low seismicity region as a ductile and unfractured block and/or a region mostly controlled by temperature and low thermochronometric ages (Boese, 2012; Bourguignon et al., 2015). Around the Whataroa Valley, several seismic phenomena such as microseismicity (Boese, 2012; Michailos et al., 2018), low-frequency earthquakes (Chamberlain et al., 2014; Baratin et al., 2018), and seismic tremor (Wech et al., 2012) have been reported.

\subsection{Data and methodology}

\subsubsection{Data}

We used 159 out of the 160 three-component stations (green circles on Figure 4.2) that were deployed as a relatively sparse array throughout the valley with an average spacing of $\sim 35 \mathrm{~m}$. The deployment was to record shots from active sources (vibroseis), as well as to continuously record passive source (earthquake) and continuous noise data, and was active from $16^{\text {th }}$ to $21^{\text {st }}$ January 2016 (6 days) (Townend et al., 2018). The 159 three-component receivers were later set up in a rolling mode (each patch was moved to a new position, and the same vibroseis positions were activated while each patch was occupied) with a spacing of $10 \mathrm{~m}$ perpendicular and $20 \mathrm{~m}$ parallel to the main strike of the Alpine Fault. The patch array was successively moved 12 times over the 5 day period of $22^{\text {nd }}$ to $26^{\text {th }}$ January 2016 to cover the central part of the valley (see odd and even Cube array, red and yellow respectively on Figure 4.2). The sensors were oriented to Magnetic North using a level bubble and a compass (Townend et al., 2018) but we corrected our measurements to reflect the True North $\left(23^{\circ}\right.$ magnetic declination around the Whataroa Valley). The initial focus for this array configuration was to construct a detailed 3D tomography of the hanging wall using active sources (Townend et al., 2018). In this Chapter, we took advantage of the few earthquakes recorded during this dense deployment (the sparse array) to characterize the anisotropy structure within 
the Whataroa Valley. In conjunction with these dense arrays, we used data from five three-component short-period $(2 \mathrm{~Hz})$ stations (blue triangles in Figure 4.2) deployed during the 2016 Whataroa Active Source Seismic Experiment (Lay et al., 2016, 2020; Townend et al., 2018), four shallow borehole ( $\sim 27 \mathrm{~m})$ short-period $(4.5 \mathrm{~Hz})$ stations (blue triangles in Figure 4.2) deployed in and around the DFDP-2 drill-site during the drilling (Chamberlain et al., 2017b), and one SAMBA station (WHAT2; Boese, 2012). Table 4.1 shows station information used for event location.

\subsubsection{Methods}

Local earthquakes recorded on the dense deployment of sensors were detected using both an energy-based method (Allen, 1978) and matched-filter detection technique (Gibbons and Ringdal, 2006) and located with the HYPOCENTER location algorithm (Lienert and Havskov, 1995). We first used the energy-based method (a ratio of shortterm average to long-term average (STA/LTA) detection algorithm; Allen, 1978), which is based on the variations in seismic amplitude, to detect possible earthquakes. The STA/LTA technique is fast and easy to implement and requires no prior event information, but can produce many false detections. We used the network trigger function available in the open source Python package EQcorrscan (Chamberlain et al., 2017a) which allows setting parameters such as trigger threshold, band-pass filter, and STA and LTA values for each individual station (Kaiser et al., 2017). Seismic noise normally varies from station to station, hence the need to use station-specific parameters for event detection. Table B.1 of Appendix B shows the parameters used for the triggering algorithm for each individual station used. We only make detections if four or more stations are triggered and are within an $8 \mathrm{~s}$ window of each other. We manually inspected the STA/LTA detections to remove all false detections and any possible explosion events from the nearby Whataroa quarry site. After removing false detections, a total of five events are retained. Figure 4.3 shows waveform examples of a detected earthquake recorded on most of the stations.

Since the energy-based detection method is likely to miss potential events due to overlapping waveforms and/or smaller magnitude events (with smaller amplitudes; 


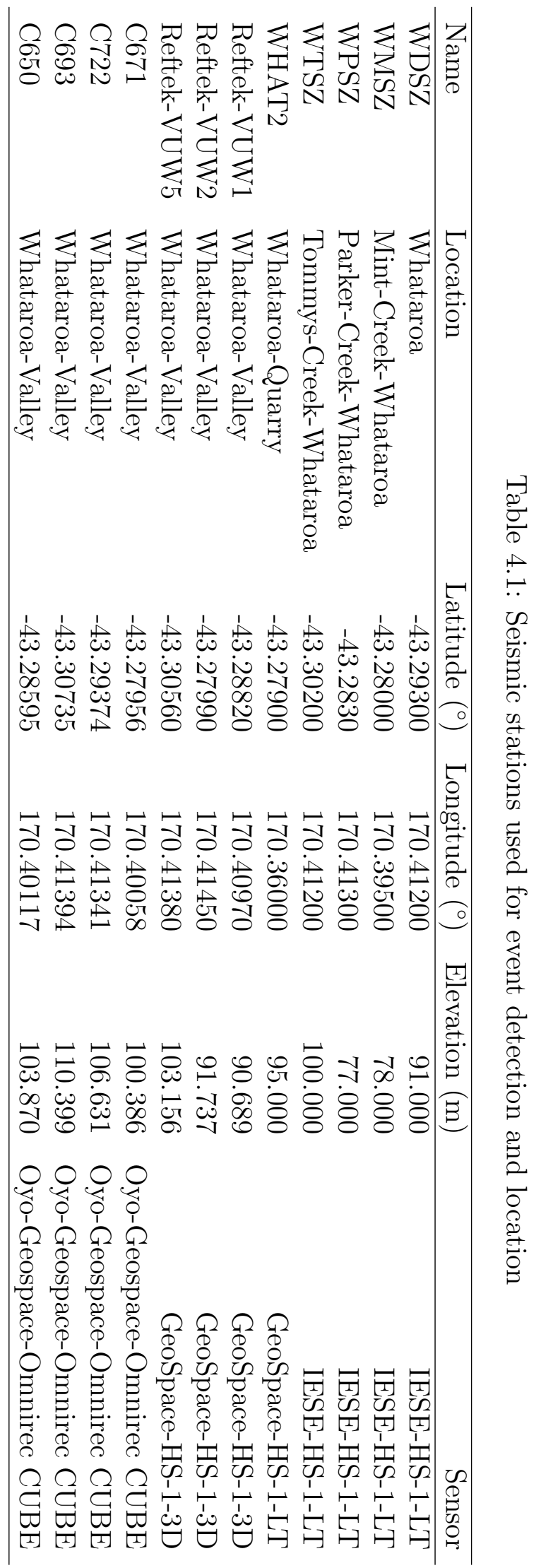


Gibbons and Ringdal, 2006; Chamberlain et al., 2017a), we used the matched-filter detection technique to detect any possible missed events. We used the open-source EQcorrscan Python package (Chamberlain et al., 2017a), which implements the crosscorrelation method of Gibbons and Ringdal (2006) to compute matched-filter detections. The five events determined using the STA/LTA technique were used as template events for the cross-correlation routine following the approach and the workflow of Chamberlain et al. (2017b) and Warren-Smith et al. (2017). We used eight stations (three Reftek stations, four DFDP-2 network stations and one SAMBA station) for matched-filter detection (see Table 4.1 and Figure 4.2). For each template, we resampled to $50 \mathrm{~Hz}$, bandpass-filtered between 1 and $10 \mathrm{~Hz}$, and use $2 \mathrm{~s}$ of the waveform starting $0.1 \mathrm{~s}$ before the phase-pick to remove any preceding random noise and scattered phases. The processed template is correlated with the daylong waveforms on all three channels at each station. The cross-correlation sum across the network is calculated by summing the computed single channel normalized cross-correlation coefficients (Chamberlain et al., 2017a). To minimize false detections, we make a detection only when the cross-correlation sum across the network exceeds a threshold value of eight times the median absolute deviation of the day-long cross-correlation sum. Detection origin times within one second of another, which are likely duplicates detections, were removed. After manually removing false detections and possible duplicate events, a total of 12 local events were retained.

We used the HYPOCENTER location algorithm (Lienert and Havskov, 1995) which is integrated into the SEISAN analysis software (Havskov and Ottemöller, 2008) and the 1-D velocity model of Boese (2012) to determine the HYPOCENTER locations. We estimated local magnitudes based on the amplitude (measured through SEISAN). For our event location, we used 12 stations; four Cube stations in addition to the 8 stations used for event detection (see Table 4.1 and Figure 4.2). Identification of correct P- and S- phase arrival times and amplitudes, which underpin the earthquake location, uncertainty, and magnitudes, were manually determined. To meet a root-mean-square (rms) travel time residual requirement of $1 \mathrm{~s}$, we used only detections recorded on at least four stations with four P-phase and four S-phase arrival times. The spatial 


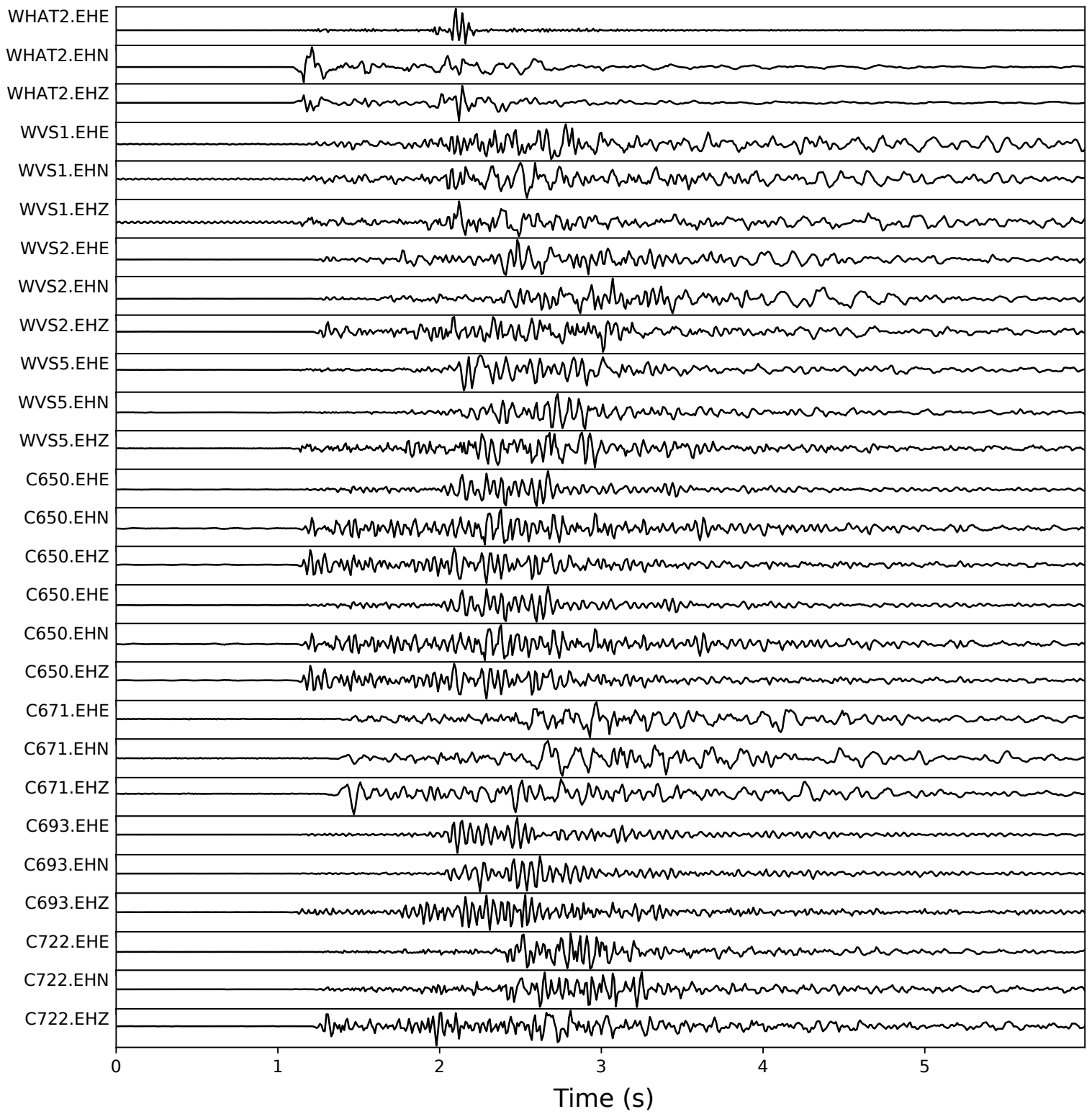

Figure 4.3: Example raw waveforms of a detected event using the STA/LTA (earthquake detected on 20/01/2016 at 04:35 UTC) used for location. They are ordered according to the station name. 


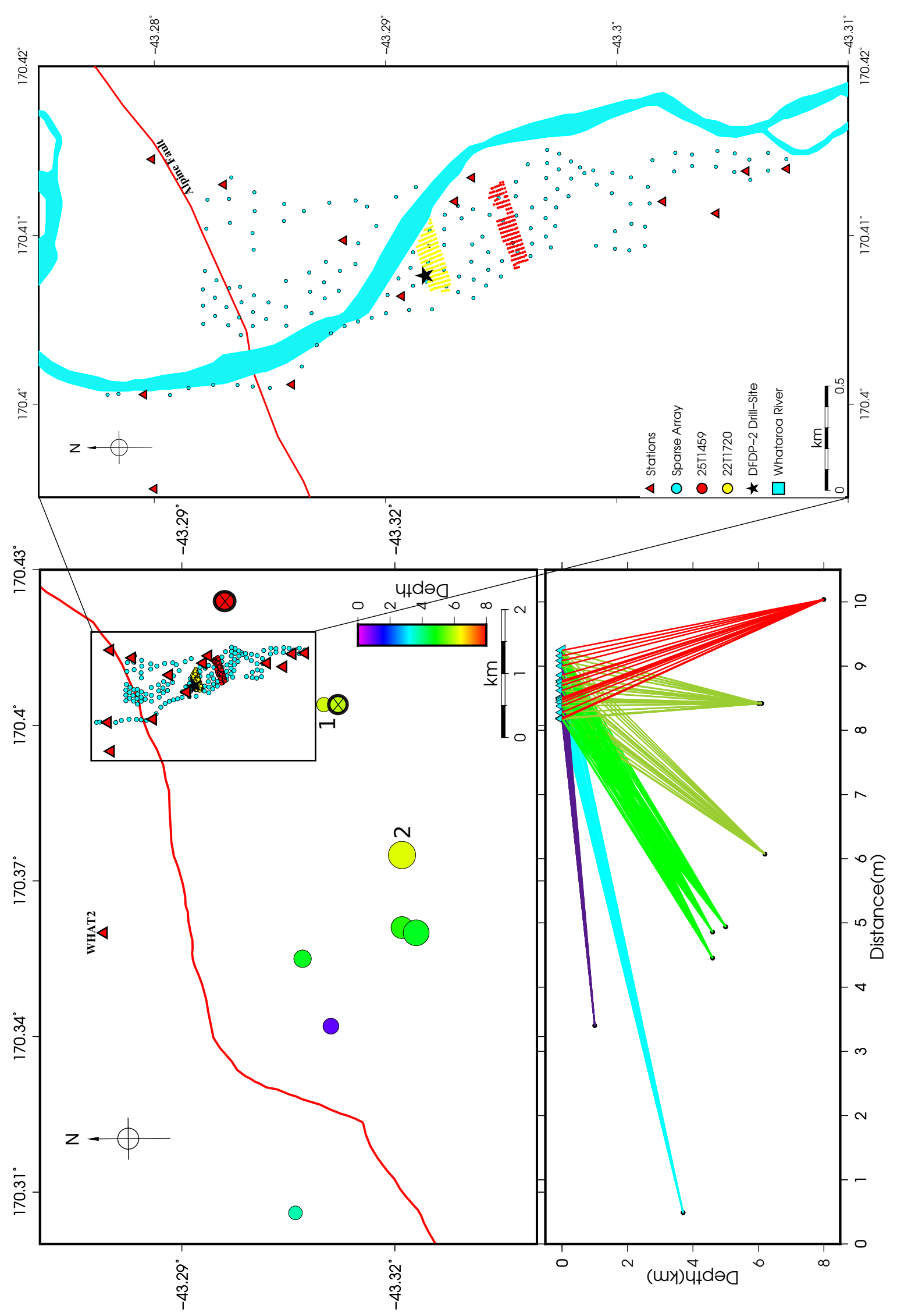

흘 골 त्ञ 엄 궁. 궁 苍唔 艺. 过 co is

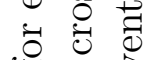
ग 至

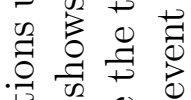

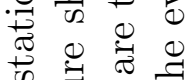
पै 800 击 满 $\circ$ ص

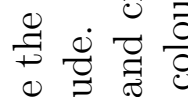
范.

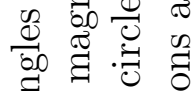
. 考荡 थी $\dot{0}$ औก भ च సี สี $\frac{0}{\mathbb{Z}} \frac{0}{0}$

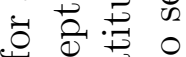
을

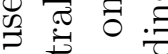

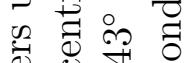

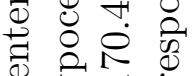

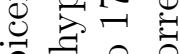
뤙 요의

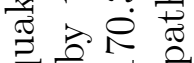
可它 đँ 0.70 पे 荥

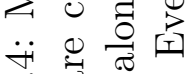

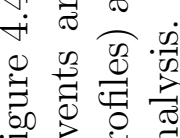

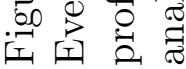


distribution of earthquakes (circles except the gray circle which are the station of the cube array) and stations (red triangles) used are shown Figure 4.4

The technique used in measuring shear wave splitting is presented in detail in Section 2.2.1. A brief overview of the method is presented here. We estimated shear wave splitting parameters using the Multiple Filter Automatic Splitting, MFAST codes (Wessel, 2010; Savage et al., 2010a) which uses a combination of the Silver and Chan (1991) eigenvalue minimization method and Teanby et al. (2004a)'s cluster analysis method that makes use of multiple time windows. The procedure is to find the splitting parameters that best remove the splitting. The splitting parameters were estimated using the eigenvalue minimization method repeated over multiple time windows around the S-phase. We filtered the waveforms with a series of band-pass filters (high-pass frequency filters were used for this analysis), and the product of the bandwidth and the signal-to-noise ratio of the filtered waveform was used to determine the best three filters (Wessel, 2010; Savage et al., 2010a). Table B.2 shows the range of filters used for this analysis. Shear waves with a signal to noise ratio (SNR) greater than 3 were used for shear wave splitting measurements. We used hand-picked shear wave arrival times for shear wave splitting measurements. The windows were automatically selected based on the dominant period around the filtered shear wave (Savage et al., 2010a). Cluster analysis over the 75 window measurements was used to select the final splitting parameter for the filter. We chose the splitting parameter pair $(\phi, \delta t)$ that best removes the splitting as measured by the smallest eigenvalue of the corrected covariance matrix, as the best measurement for the given time window (Savage et al., 2010a). The splitting parameters estimated were graded from A to D depending on the consistency between the $\phi$ and $\delta t$ measurements in the different windows. We used the MFAST version designed for very local earthquakes. Wessel (2010), Savage et al. (2010a), Walsh et al. (2013) and Section 2.2.1 give a detailed description of the MFAST method.

To ensure we were using high-quality measurements, all null graded measurements (events for which no measurable splitting occurs or events for which $\phi$ is within $20^{\circ}$ of parallel or perpendicular to incoming polarization orientation) were removed and we selected only A and B graded measurements. To avoid the complexity of converted 

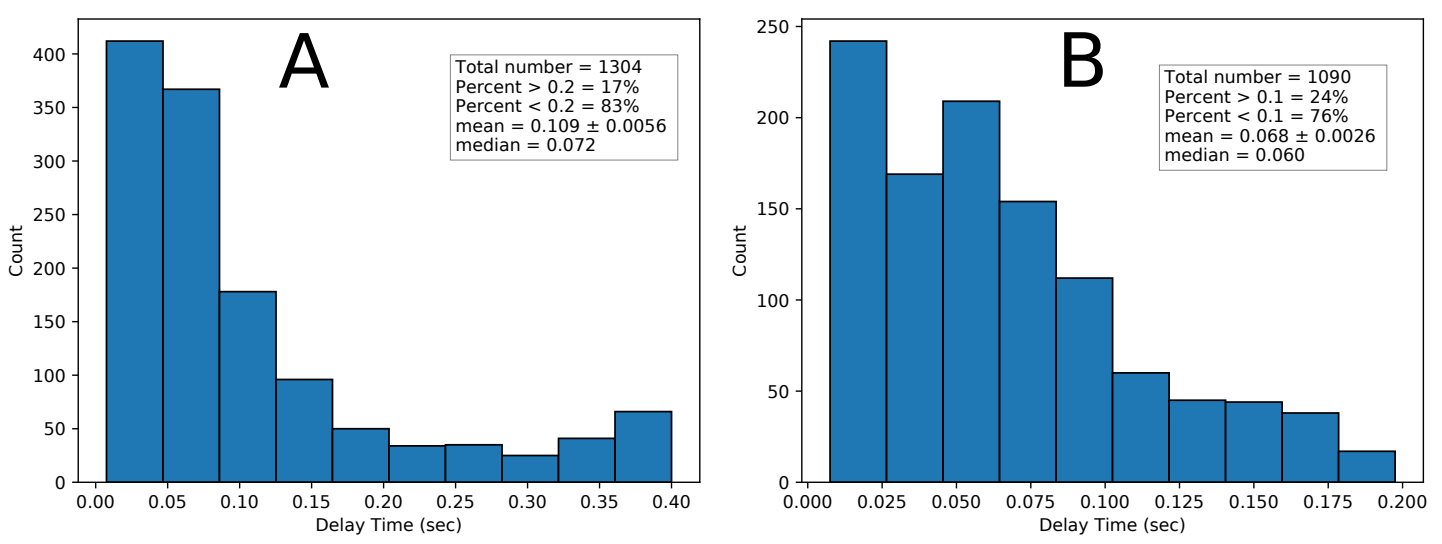

Figure 4.5: Distributions of delay times. A: All AB graded measurements B: AB graded measurements with delay time less than or equal to $0.2 \mathrm{~s}$.

phases resulting from refraction/reflection and to ensure near-vertical wave propagation, only measurements with an incident angle less than $35^{\circ}$ were used. Incidence angles were estimated using the TauP toolkit (Crotwell et al., 1999) and a 1D velocity model from Boese (2012). We also limited maximum delay times, $\delta t$, to $0.2 \mathrm{~s}$ because more than $80 \%$ of the $\delta t$ measurements were below 0.2 (see Figure 4.5 ) and moreover, local crustal events are often not expected to have larger delay times (Balfour et al., 2005; Cochran and Kroll, 2015). Following the quality control, a total of 1090 (out of 1304) high-quality measurements were obtained. Because the sensors were oriented to the Magnetic North, we rotated all our fast direction measurements to True North by subtracting $23^{\circ}$ (magnetic declination around the Whataroa Valley; NCEI Geomagnetic Modeling Team and British Geological Survey, 2019) from the fast direction measurements and the incoming polarisation.

We also estimated percentage anisotropy, $\kappa$, following the approach of Babuska and Cara (1991) and Savage (1999). Here we define $\kappa=\left(v_{\max }-v_{\min }\right) / \bar{v}$, where $v_{\max }$ is velocity of the shear wave along the fast orientation, $v_{\min }$ is velocity of the shear wave along the slow orientation and $\bar{v}$ is an average of $v_{\max }$ and $v_{\min }$. Assuming both fast and slow waves travel with the same path length, $\kappa$ can be related to $\delta t$ as: $\kappa=200 * \delta t /\left(2 t_{s}+\delta t\right)$. We use $\kappa$, the percentage anisotropy as well as $\delta t$, to characterize the anisotropy structure.

O'Connell and Budiansky (1974) define apparent crack density as $\epsilon=N a^{3} / V$, 
where $a$ is the radius of the crack and $N$ is the number of cracks in a volume, $V$. Following the approach of Hudson (1981), Leary et al. (1990), and Unglert et al. (2011) (simplifying O'Connell and Budiansky (1974)'s definition and relating $\delta$ t to crack den-

sity), we estimated the apparent crack density, $\epsilon$ using $\epsilon=\xi(\delta t / L)$. Here $\xi$ is the shear velocity of the medium, $L$ is length of the ray-path in the anisotropic medium. We assumed $L$ to be the source-receiver path-length, since our waveforms propagate through the first $8 \mathrm{~km}$ of the crust, which is often considered as anisotropic (Savage, 1999; Crampin and Gao, 2006). Based on the 1-D velocity model of Boese (2012) around the Whataroa Valley, we chose $\xi$ to be $3 \mathrm{~km} / \mathrm{s}$, which is the average shear velocity of the first $8 \mathrm{~km}$ of (Boese, 2012)'s 1-D crustal velocity model. For each high quality SWS measurement, we estimate the implied crack density.

\subsection{Results}

\subsubsection{Event locations}

Out of the 12 detected events only 9 events were successfully located with rms values less than $1 \mathrm{~s}$ (Figure 4.4). For this study, precise relative location and relative magnitude are not a priority, since they have no significant effect on the shear wave splitting analysis. The event depths ranged between 1 and $8 \mathrm{~km}$ with an average of $4.0 \mathrm{~km}$, which is consistent with the depth of micro-seismicity around the region (Boese, 2012; Chamberlain et al., 2017a; Michailos et al., 2018). Local magnitudes estimated using the HYPOCENTER location algorithm (implemented in SEISAN), were between 1.0 and 3.6, which are also consistent with previous study (Michailos et al., 2018).

\subsubsection{General overview of SWS measurements}

A total of 1090 (out of 1304) high-quality measurements (following the quality control criterion described in Section 4.2.2) were obtained from the 159 stations using seven events (recorded by the sparse stations) were obtained from the 159 stations using seven events (recorded by the sparse stations). The two other events (the black circled 
and crossed circles on Figure 4.4), that were recorded only on the patch array, were not used for this analysis (due to the different stations location).

The summary of anisotropy parameters, percentage anisotropy and crack density with their corresponding standard errors for each station are presented in Table B.3 to B.6 of Appendix B.

Fast direction results for each station are shown as rose diagrams in Figure 4.6. The regional $S_{H \max }$ orientations from (Leitner et al., 2001; Boese, 2012; Michailos et al., 2020) are also shown on Figure 4.6 as green bow-ties with wedges showing the 95\% confidence interval. Plots of individual fast directions for individual events are shown in Figure B.1 to B.7 of Appendix B. We observe a significant scatter in the rose diagrams. To obtain more reliable estimates of $\phi$ orientations and to minimize the observed scatter, we estimated the circular mean of $\phi$ orientation at each station. The circular averages of $\phi$ were estimated using a circular statistics approach (Berens, 2009) and are presented (Figure 4.7) only if the standard deviation of fast orientations at each station is less than $30^{\circ}$ and the standard error of the mean is less than $10^{\circ}$. Figure 4.7 shows the spatial distribution of averaged $\phi$ orientation, represented as bowties, at each station. The wedges of the bow-ties show the standard error of the mean estimate at each station. Although there is scatter in the rose diagram plot (Figure 4.6) at some stations, the circular averaging at individual stations (Figure 4.7) revealed a general spatial pattern of the fast direction with most of the stations showing a NE-SW orientation. The overall mean orientation of the fast direction is $47 \pm 23^{\circ}$, which is near parallel to the strike $\left(\sim 55^{\circ}\right)$ of the Alpine Fault trace in the valley, the orientations of regional and borehole foliation planes ( $\sim 53^{\circ}$; Little et al., 2002; Gillam et al., 2013; Townend et al., 2017) and the fracture orientations $\left(\sim 57^{\circ}\right)$ obtained from acoustic borehole imaging of the DFDP-2 (Townend et al., 2017).

The mean $\delta t$ values at individual stations range from $9 \mathrm{~ms}$ to $186 \mathrm{~ms}$ with a mean value of $70 \pm 3 \mathrm{~ms}$. Percentage anisotropy, $\kappa$ range from 0.21 to $4.79 \%$ with a mean value of $1.67 \pm 0.07 \%$. To further investigate the spatial distribution of $\delta t$ and strength of anisotropy, we plotted the average station $\delta t$ and percentage of anisotropy at each station (Figure 4.8). The spatial distributions of both $\delta t$ and percentage of anisotropy 


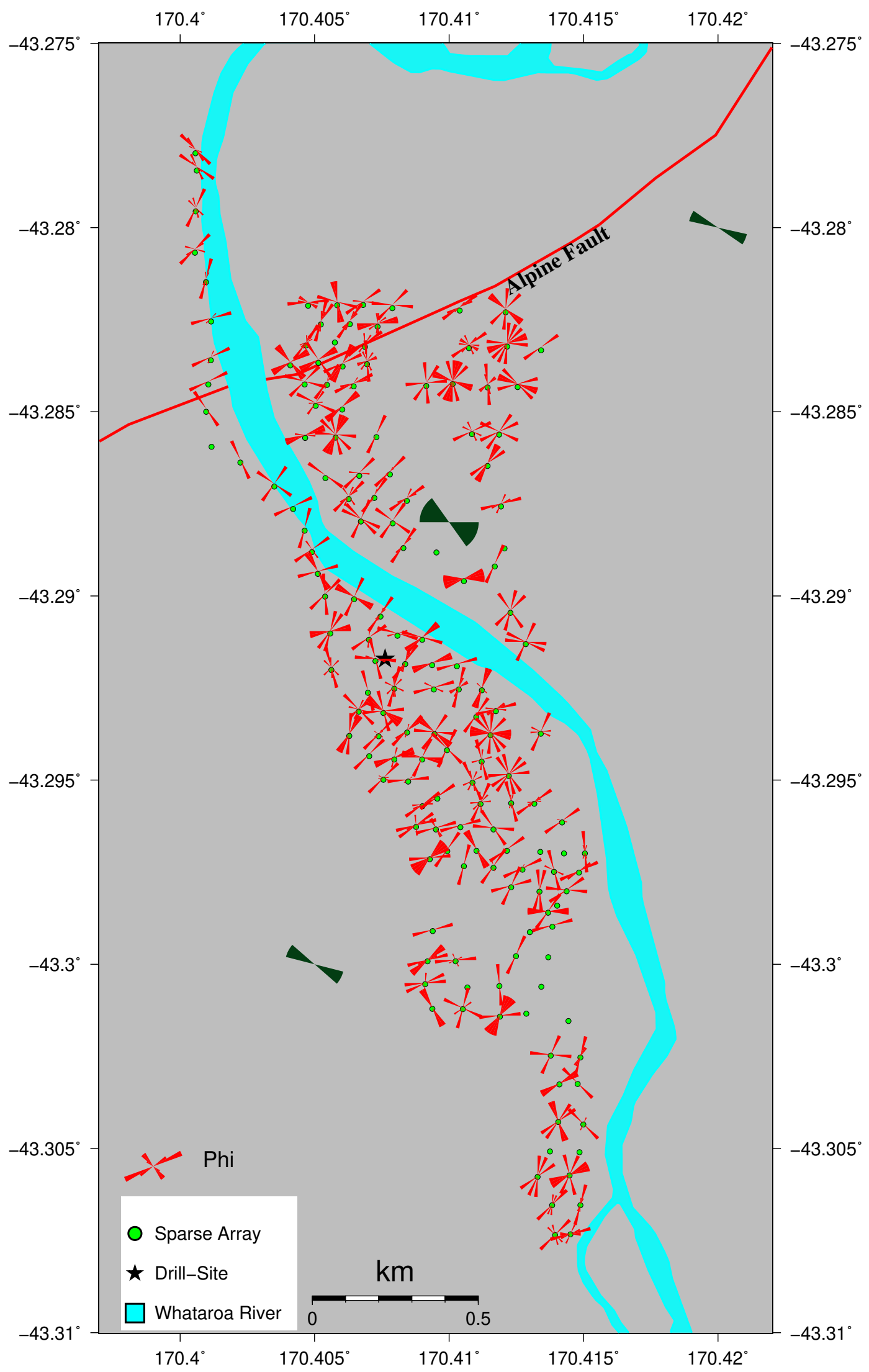

Figure 4.6: The spatial distribution of $\phi$ orientation (AB grade) obtained from local S-phase events is plotted as circular histograms (rose diagrams) the stations at which measurements were made. The red line shows the Alpine Fault trace. The green bowties shows the regional $S_{H \max }$ orientations from Leitner et al. (2001) (bottom left), Boese (2012) (top right) and Michailos et al. (2020) (middle). 
at each station revealed no clear pattern (Figure 4.8). The average crack density is $0.06 \pm 0.02$ and ranges from 0.006 to 0.242 . 


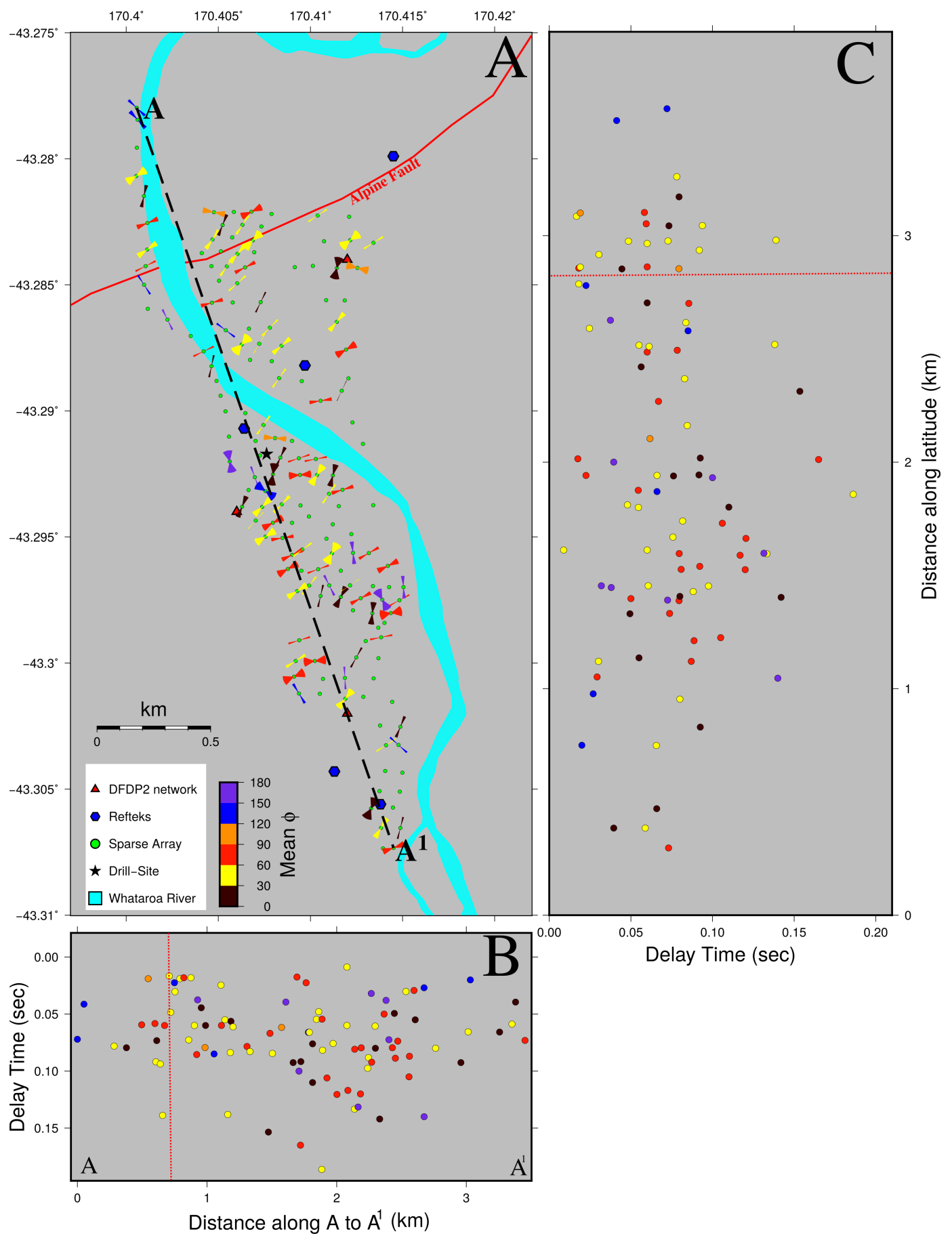

Figure 4.7: A. The spatial distribution of averaged $\phi$ orientations obtained from local events is plotted as bow ties with the wedges showing the $95 \%$ confidence intervals. The red line shows the Alpine Fault trace. We plot only measurements with a standard deviation of less $30^{\circ}$. Cross-sections $\mathrm{B}$ and $\mathrm{C}$ show the variation of the delay time measurements projected onto line $\mathrm{A}$ to $\mathrm{A}^{1}$ of Figure 4.7 (a perpendicular line across the Alpine Fault) and the latitude (from -43.275 to -43.31 ) respectively. The colour of the dots represents the orientation of $\phi$. The red vertical and horizontal dotted lines on $\mathrm{B}$ and $\mathrm{C}$ respectively are the position of the Alpine Fault. 

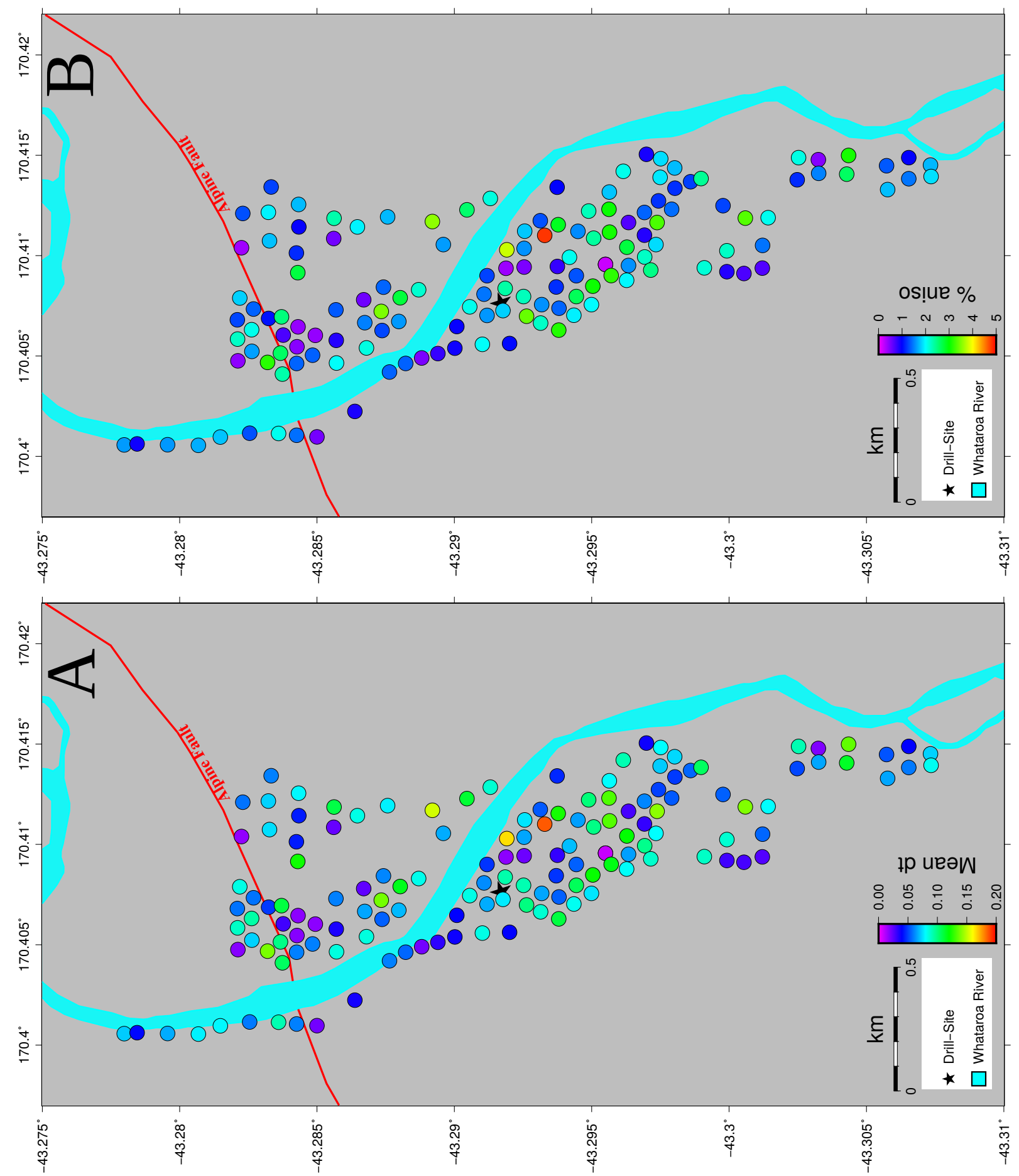

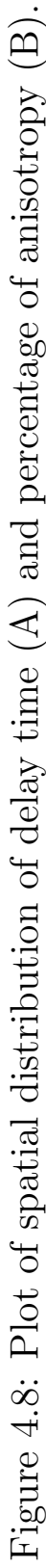




\subsection{Discussion}

The low level of seismicity (also referred to as "seismic gap") that we observed has been reported by earlier researchers (Leitner et al., 2001; O'Keefe, 2008; Boese, 2012; Bourguignon et al., 2015; Chamberlain et al., 2017a; Michailos et al., 2018). Boese (2012) attributed the "seismic gap" in the region to a ductile and unfractured block that may be controlled by high temperature with low thermochronometric ages. The observed mean $\delta t$ value of $70 \pm 3 \mathrm{~ms}$, is consistent with Pulford et al. (2003)'s measurements. Using active source data, Pulford et al. (2003) observed similar delay times $\sim 80 \mathrm{~ms}$ around the most deformed part of the Alpine Fault region (Pulford et al., 2003, similar frequency but potentially different refions; ). These observed average percentage of anisotropy and crack density are small compared to crustal anisotropic studies close to other faults (e.g. Zinke, 2000; Mishra and Zhao, 2003; Cochran et al., 2006)

Although we observe scatter in the $\phi$ orientations at individual stations, the general trend $\left(47 \pm 23^{\circ}\right)$ closely follows the strike of the Alpine Fault and the orientations of the foliation plane (Norris and Cooper, 2001; Norris and Toy, 2014; Little et al., 2002; Langridge et al., 2018; Townend et al., 2018; Massiot et al., 2018). The fact that $\phi$ orientations around the fault zone are nearly parallel to the strike of the Alpine Fault and the foliations probably reflects the effect of the deformation in the fault zone or is fabric-related anisotropy (aligning with the preferred mineral orientation), or both. A similar conclusion holds for analysis of crustal anisotropy around the North Anatolian fault, where Peng and Ben-Zion (2004, 2005) observed fault-parallel $\phi$ orientations and suggested shear fabric as possible sources for the observed anisotropy near the fault (Peng and Ben-Zion, 2004, 2005). The effect of foliated rocks on SWS measurements has been observed empirically and numerically. Barruol and Mainprice (1993) used numerical modelling to evaluate the effect of foliated rocks on SWS and observed a strong correlation between the variation of the foliation and large delay times (Barruol and Mainprice, 1993). They further observed that the fast direction is near parallel to the orientation of the foliation plane. The observed NE-SW trend of the fast direction is perpendicular to the orientation of the regional $S_{H \max }$ direction. This suggests that 
the observed anisotropy is not induced by tectonic stresses at earthquake depths.

Perhaps, the topographic contrast around the valley contributes to the observed anisotropy as reported in the anisotropy studies in other regions (e.g. Chapter 3 Araragi et al., 2015; Illsley-Kemp et al., 2017). We cannot quantitatively compare orientations of $\phi$ and variable orientation of gravitationally induced stress, $S_{H_{\max }}^{G r a v}$ in this region as was done in Section 3.5.5 of Chapter 3 because these methods rely on larger scale crustal structures on the order of $30 \mathrm{~km}$ (Hirschberg et al., 2018). In the Hirschberg et al. (2018) implementation, the gravitational potential energy (which is used to estimate $S_{H_{\max }}^{G r a v}$ ) is averaged over a crustal thickness (on the order of kms; $\sim 30 \mathrm{~km}$ ) and is estimated at a grid point spacing of similar order as the crustal thickness $(\sim 30 \mathrm{~km}$; Hirschberg et al., 2018). In this study our measurements are on the order of meters and hence below the resolution of $S_{H_{\text {max }}}^{G r a v}$.

The low delay times we observed in the presence of strongly foliated Alpine Fault mylonites (considered as a source of crustal anisotropy with large delay times; Okaya et al., 1995; Godfrey et al., 2000; Dempsey et al., 2011; Simpson et al., 2020; Adam et al., 2020) are likely due to the high-frequency waveforms used here (short period stations). Pulford et al. (2003) also observed similar small delay times around the most deformed part of the Alpine Fault region and attributed it to the use of high-frequency waveforms (with small wavelengths). Although several laboratory and numerical models of sampled rocks of the Alpine Faults (e.g. Okaya et al., 1995; Dempsey et al., 2011; Simpson et al., 2020; Adam et al., 2020) have report on high percentage of anisotropy, the low percentage of anisotropy reported in this studies are probably due to the different wavelengths used in both the laboratory and numerical models studies and the wavelengths used in this study. In this study, our wavelengths are approximately 127 m. This is based on the dominant frequencies of $\sim 8 \mathrm{~Hz}$ of the shear waveforms and low shear velocity of $\sim 1080 \mathrm{~m} / \mathrm{s}(M c N a b, 2017)$. This is often refered to as frequency dependency of the splitting measurements. The high-frequency waveforms used in measuring shear wave splitting may be sampling smaller features rather than the highly foliated fault zone. Also the $8 \mathrm{~Hz}$ dominant frequency also makes it difficult to measure splitting of $>1$ period (can resolve splitting less than $\sim 0.125 \mathrm{~s}$ ). As discussed Section 
1.3.1 and Figure 1.3, anisotropy exists at all scales and degrees of anisotropies often reported are dependent on the wavelength used in sampling the anisotropic medium.

The low values of delay time we observed can be related to the ductile and unfractured block, which is surrounded by a high attenuation zone, as suggested by microseismicity studies by Boese (2012) in and around the Whataroa Valley. The ductile and unfractured block hypothesis can explain the average low strength of anisotropy and crack density we observed $(1.67 \pm 0.07 \%$ and $0.06 \pm 0.02$ respectively) as well as the fast direction not being parallel to $S_{H_{\max }}$. This is because the upper crust, where the confining pressures are lower (allowing cracks to remain open), is often suggested to have some of the highest strength of anisotropy (Hudson, 1981; Aster et al., 1990; Crampin, 1984; Cochran et al., 2006; Boness and Zoback, 2006). Moreover, rocks that have been highly fractured or damaged due to faulting are considered to have a higher degree of anisotropy (Aster et al., 1990; Crampin, 1984; Cochran et al., 2006; Boness and Zoback, 2006). Mishra and Zhao (2003) observed a higher percentage of anisotropy around damaged rocks near the intersection of two faults. Thus, one will expect to observe a high degree of anisotropy as an indicator of the Alpine Fault's damage zone. However, the small degree of anisotropy and crack density we observed supports the ductile and unfractured block hypothesis and also the fact that we are observing foliation and fault fabrics rather than cracks.

\subsubsection{Spatial variability in seismic anisotropy within the valley}

To answer the question of whether a significant spatial variation in the observed anisotropy exists as we cross the Alpine Fault from the footwall to the hanging wall, we carried two averaging analyses; (i) a moving distance averaging of the anisotropic parameters along line $\mathrm{A}$ to $\mathrm{A}^{1}$ with a window of $200 \mathrm{~m}$ and shifting the window forward by $1 \mathrm{~m}$ for each estimate and (ii) averaging fast directions every $200 \mathrm{~m}$ along line A to $\mathrm{A}^{1}$ of Figure 4.7 (a perpendicular line across the Alpine Fault). We projected all 1090 measurements from the 159 stations onto the perpendicular line across the Alpine Fault (A to $\mathrm{A}^{1}$ on Figure 4.7 ) to estimate the distance.

We performed a simple moving distance average with a $200 \mathrm{~m}$ window as a smooth- 

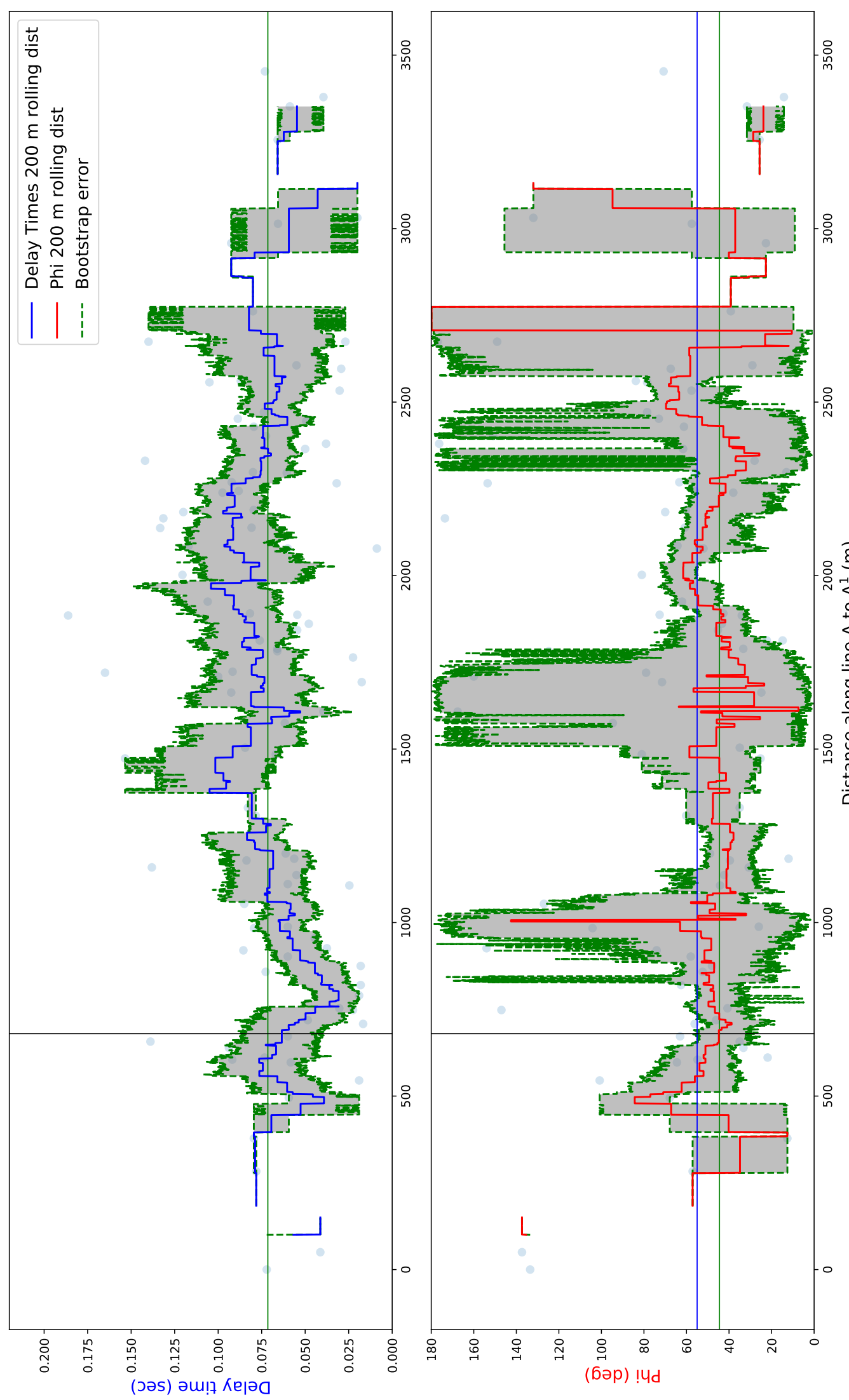

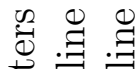

西

त्ञ

政

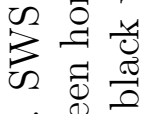

$\dot{0} 50$

$\stackrel{\square}{ }$

$\ddot{\theta} \dot{0}$

$\lambda$.

远

bo $\overrightarrow{0}$ 红

贯

要 80.

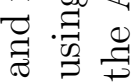

客它㟧

要

范要

蒫.

类安

$\because$ o

g

유윰

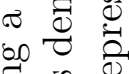

.

$\theta=\frac{0}{2}$

]

$50 \pm$

苟声

密要导

능

0.00

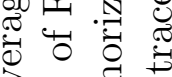

专

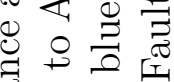

造

氜 可

bo

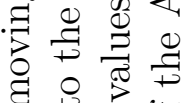

节 素

可志

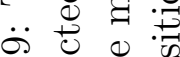

ㅁ.

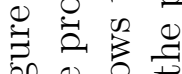

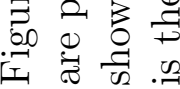


ing function for the SWS measurements, with the aim to minimize the observed scatter in our measurements as much as possible. For each moving point average, we estimated 95\% confidence intervals by using the non-parametric bootstrap approach described in Section 3.3.2 of Chapter 3. Figures 4.9 and B.8 shows a plot of moving distance averages for the SWS parameters projected unto line $\mathrm{A}$ to $\mathrm{A}^{1}$ and the latitude (from $-43.275^{\circ}$ to $\left.-43.31^{\circ}\right)$ respectively. Generally, we do not observe any significant departure of either delay time or fast direction from the mean values as we move perpendicularly along the Alpine Fault, suggesting an insignificant spatial variability in seismic anisotropy within the Whataroa Valley.

In non-overlapping $200 \mathrm{~m}$ bins along line $\mathrm{A}$ to $\mathrm{A}^{1}$, we averaged the orientations of the fast direction and estimate the $95 \%$ confidence intervals. Figure 4.10 shows the plot averaged $\phi$ orientations. The average values are plotted at the center of the bin as bow ties with the wedges representing the $95 \%$ confidence intervals. We also show a rose diagram (white wedges on Figure 4.10) of all measurements in the bin as a baseplot for the bow ties. Generally we observe a consistent NE-SW direction as we move perpendicularly across the fault from the footwall to the hanging wall. This also suggests that there is no significant spatial variation of the observed anisotropy in the Whataroa Valley.

Ideally, one may expect that a ray path that samples the hanging wall at the end of its path will have a larger percentage of its anisotropy from the hanging wall than one that samples the footwall at the end of its path. Delay time (used in calculating percentage of anisotropy) is an integrated quantity that accumulates along the ray path and it is dependent on the length of the ray path (Rümpker and Silver, 1998; Savage, 1999; Saltzer et al., 2000). The path length of the entire ray path is usually used to calculate the percentage of anisotropy. Calculating the percentage of anisotropy for the last part of the path (often the last layer) depends on how the anisotropy changes as it goes towards the surface. If the anisotropy changes slowly, the first arriving wave may only rotate the directions (fast direction), keeping the delay time that accumulates throughout the path (Rümpker and Silver, 1998; Saltzer et al., 2000). If it changes rapidly with a large enough difference in the two layers, it may simply split twice, and 


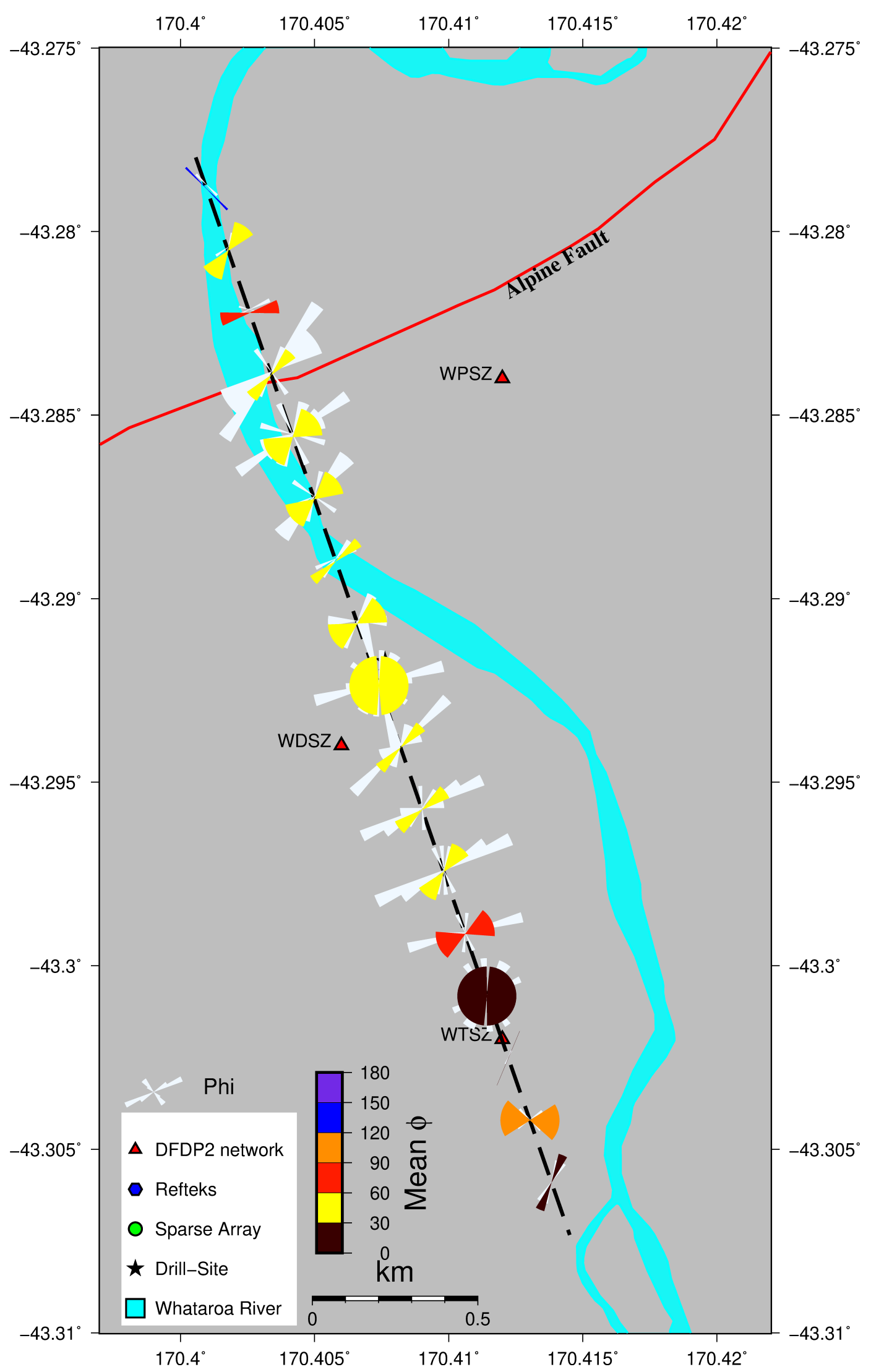

Figure 4.10: The spatial distribution of averaged $\phi$ orientations every $200 \mathrm{~m}$ across the Alpine Fault. The average $\phi$ orientations obtained from local S-phase events are plotted as bow ties with the wedges showing the $95 \%$ confidence interval. The bow ties are superimposed on the rose diagram plot of measurements in the moving average bin. The red line shows the Alpine Fault surface trace. 
one may only measure the last layer (Silver and Savage, 1994). In the latter case, it will appear to be a smaller anisotropy if one uses the entire path to calculate the percent.

However, to know exactly where anisotropy occurs along the ray path, one would need to use raytracing or better yet, full waveform forward modelling, to know how the waveform will split on its way to the surface. Since the last part of the path has the most effect on anisotropy, it will be the most important for the orientation (Rümpker and Silver, 1998). But such raytracing cannot help to determine the anisotropy in each layer unless the splitting in each part of the block is taken into account. The use of raytracing analysis to examine which event (ray) sampled the foot-wall or the hanging wall (or spend the most time in either layer) may help in explaining the anisotropy structure as one move across the Alpine Fault. But to explain the splitting measurements within the Whataroa Valley, a full 3D ray tracing with a good velocity model combined with a determination of the splitting in each section of the ray path, a well-constrained earthquake depth, and a well-constrained location of Alpine Fault is warranted. These analyses are beyond the scope of this study.

\subsubsection{Mechanisms of anisotropy in the Whataroa Valley}

To further examine the hypothesized mechanisms controlling the observed anisotropy in the valley, we compared the mean orientation of $\phi$ with structural data sets. Figure 4.11 shows a lower hemisphere plot of the orientations of the fast direction, $S_{H \max }$ and other structural data sets within and around the Whataroa Valley. For ease of comparison, we used a dip of $50^{\circ}$ to show $\phi$ measurements on the stereonet plot (Figure 4.11). Clearly, the mean fast directions observed (red thick line on Figure 4.11) are near-parallel to the orientations of fractures and foliations obtained from the acoustic borehole televiewer installed in the DFDP-2 (Townend et al., 2017), the orientation of foliations measured from the Whataroa Valley (Little et al., 2002) and Tatare Stream (Gillam et al., 2013) outcrops and the orientation of the Alpine Fault trace (Norris and Cooper, 2001; Norris and Toy, 2014; Little et al., 2002; Langridge et al., 2018). We suggest that the mechanism that controls anisotropy in the Whataroa Valley in the vicinity of the Alpine Fault is mainly structural, hence the hypothesis of structurally 


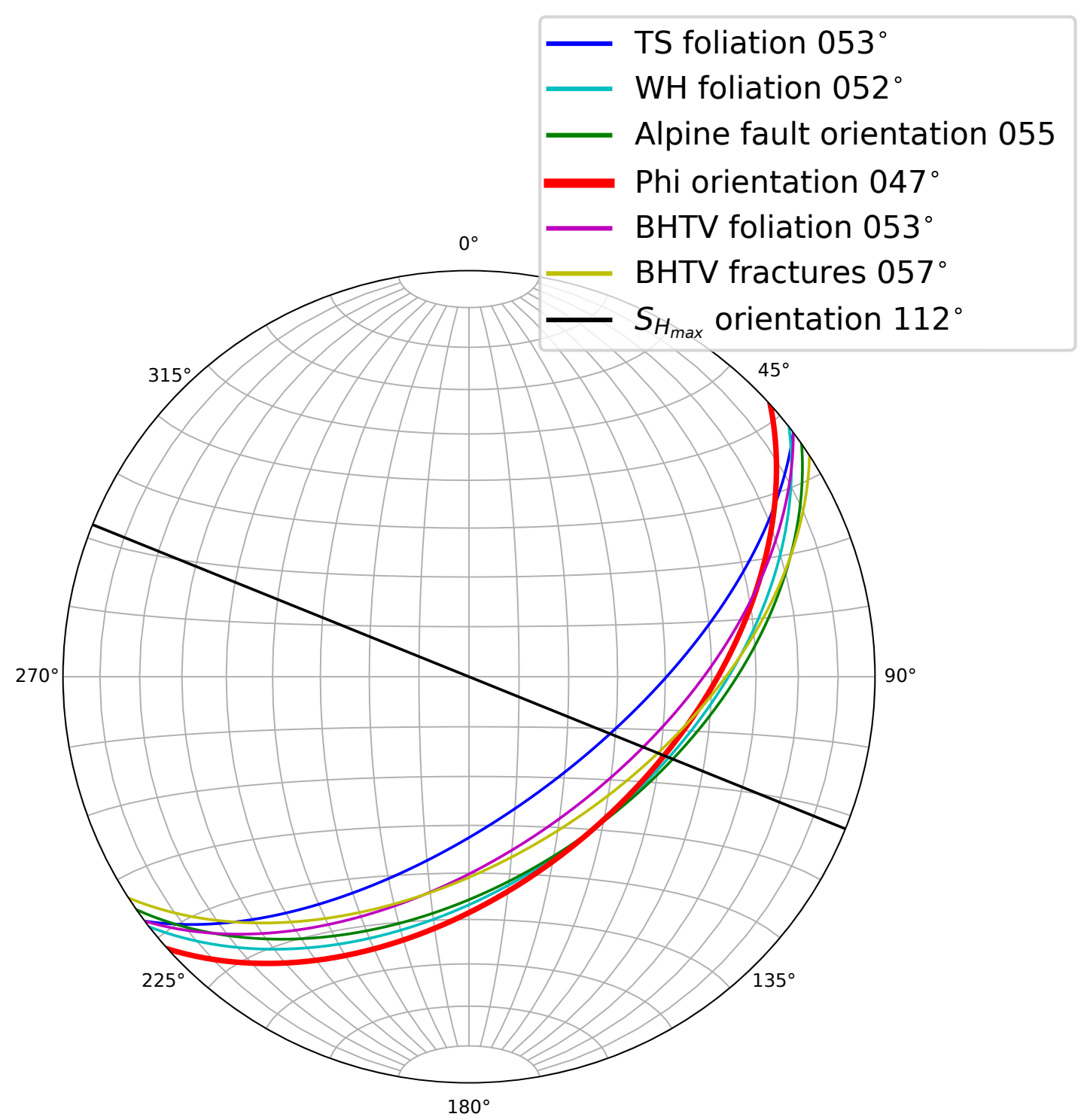

Figure 4.11: Lower hemisphere, equal-area stereonet showing orientations of $\phi, S_{\text {Hmax }}$, foliation, fractures, and the Alpine Fault. WH and TS represents foliations of Whataroa Valley (Little et al., 2002) and Tatare Stream (Gillam et al., 2013) outcrops respectively. BHTV foliation and fractures are structural information obtained from the acoustic borehole televiewer installed in the DFDP-2 (Townend et al., 2017). For ease of comparison, we used a dip of $50^{\circ}$ to show the $\phi$ measurements on the stereonet plot. 
controlled anisotropy best explains our results. Several researchers have reported on similar observations where $\phi$ orientations measured on stations close to faults are consistently fault-parallel (e.g. Zinke, 2000; Matcham et al., 2000; Balfour et al., 2005; Cochran et al., 2006; Boness and Zoback, 2006). They often invoke the structural control hypothesis as the mechanism controlling anisotropy in such regions.

The orientation of $S_{H \max }$ is at a high angle to the mean fast directions, which indicates that the anisotropic mechanism in the valley is likely not related to the stress at the depth of the earthquakes, hence the stress-controlled hypothesis, where cracks open or close in a preferred orientation, would not explain our results. Okaya et al. (1995) also suggested that cracks could not explain the anisotropy they observed with the Alpine Fault schist.

\subsection{Conclusions}

We have presented a detailed spatial analysis of seismic anisotropy within the Whataroa Valley in the vicinity of the Alpine Fault using a dense deployment of 159 threecomponent stations with an average spacing of $\sim 35 \mathrm{~m}$. The overall mean orientation of the fast direction was $47 \pm 23^{\circ}$, which is near parallel to the strike $\left(\sim 55^{\circ}\right)$ of the Alpine Fault trace in the Whataroa Valley and the orientations of regional and borehole foliation planes. A mean delay time, $\delta t$, of $70 \pm 3$ ms was observed, which is consistent with a small amount of splitting of the high-frequency phases (dominant frequencies $8.45 \pm 0.08 \mathrm{~Hz}$ ). We showed that there was no significant spatial variation of the shear wave splitting parameters as we move across the Alpine Fault trace from the hanging wall to the footwall. We conclude that the anisotropic structure in the valley is structurally controlled by the Alpine Fault fabric and foliations within the valley and that there is no significant spatial variation. Our data is sufficient to show the lack of spatial variation of the anisotropy within the Whataroa Valley, but lacks the potential to constrain the depth of the anisotropic source due to the short period of deployment and the small events detected. 


\title{
5 What Causes the Variations in Shear Wave Splitting Measurements?; examples from simulation studies
}

\begin{abstract}
For the past three decades, shear wave splitting (SWS) measurements of crustal earthquakes have been utilised as a potential technique to infer in-situ properties and to characterize seismic anisotropic structures. Often, interpretation of SWS measurements from small earthquakes is challenging and sometimes ambiguous. Earlier empirical studies have acknowledged this and suggested physical mechanisms such as scatterers, the incidence angle of the incoming shear wave, and variation in source mechanism as the likely causes. In this on going research, we simulate waveforms propagating through an anisotropic medium (using both 1-D and 3-D techniques) for a variety of scenarios, to investigate the effects that these suggested physical mechanisms have on SWS measurements. We observe that for incoming shear waves with a large angle of incidence $\left(>35^{\circ}\right)$, the estimated SWS parameters are unstable and unreliable. The variation of source mechanism showed less significant effect on SWS measurements, except for null measurements (when the incoming polarization direction is parallel to either the fast or slow direction). We observe a significant variation in estimated SWS parameters when we introduce an anomalous elastic black (scatterer) into the medium. SWS measurements and waveforms from stations at the edge of a scatterer showed more unstable results and distorted waveforms respectively, compared to stations directly above the anomaly and far away from the anomaly. We also observe that the effect of a scatterer depends on the wavelength of the incoming shear waves. For longer
\end{abstract}


wavelengths (compared to the size of the anomaly), the wave considers the medium as homogeneous. Based on our observations, we suggest that only non-null SWS parameters with angles of incidence less than $35^{\circ}$ should be considered for interpretation, as measurements outside these are unstable. Also, care needs to be taken when interpreting temporal variations of SWS measurement since spatial variations such as scatterers have significant effect on SWS parameters that can be mistaken for temporal variations. For temporal variation interpretations, we recommend using events with a similar source-receiver propagating path (repeating or near repeating events). These sample a similar medium on the propagating path of the medium and will have more stable, repeatable results, and are therefore sensitive to genuine temporal variations in the medium.

\subsection{Introduction}

The ability to monitor variations of in-situ properties, such as stress and crack density and orientation, within the Earth's crust can assist in hazard analysis and resource extraction (Crampin and Booth, 1989; Miller and Savage, 2001; Gerst and Savage, 2004; Crampin et al., 2015; Savage et al., 2015, 2016). Measuring seismic anisotropy through shear wave splitting (SWS), for the past three decades has shown potential as a tool to infer these in situ properties. The full potential of this tool has yet to be realized in part because measurements of SWS from local earthquakes give varying results and are sometimes challenging to analyse and interpret (Crampin and Gao, 2006; Johnson et al., 2010; Walsh et al., 2013). Many researchers, through empirical studies, have suggested possible physical mechanisms such as scatterers and variation in source mechanism as well as temporal changes in stress as the cause of these varying results (e.g.; Crampin, 1981; Booth and Crampin, 1985; Liu and Crampin, 1990; Crampin and Gao, 2006; Johnson et al., 2010; Walsh, 2012; Savage et al., 2015). However, there are few studies that have been carried out to investigate precisely how these mechanisms affect SWS measurements. In this Chapter, we investigate the effect that these putative physical mechanisms have on SWS measurements through numerical simulation 
(creating synthetic seismograms), using both 1-D and 3-D techniques. We explore:

(1) the effect of incidence angle of the incoming shear wave (the optimum shear wave incident angle that is allowed for SWS analysis) on SWS measurements (2) the effect of different source orientation on SWS measurements (3) the effect of scatterers on SWS measurements. This is done with the ultimate aim of improving the interpretation of SWS measurements.

The term "scattering" used here refers to the interaction of seismic waves with an anomalous body (a feature with elastic properties that differ from its surroundings). Scattering is normally observed whenever there are material changes (heterogeneity) in the wave's propagating path. A seismic wave will scatter when it encounters a discontinuity in the medium (Groenenboom and Snieder, 1995; Sato et al., 2012; Shearer, 2015). The discontinuities (scattering) referred to here are any changes in the elastic properties and/or the geometry of the medium, such as cavities, joints, inclusions, and free surfaces of underground structures. It could be that (in the shallow crust) the distributions of fluid-filled cracks, microcracks, and pore-space (having different elastic properties) can function as scatterers and have a first-order effect on the propagating shear-wave. Scattering effects can manifest in various forms, such as reflection, refraction, and diffraction (Groenenboom and Snieder, 1995; Sato et al., 2012). Scatterers can contaminate the shear wave (contamination from P-coda; Sato et al., 2012) and may obscure the detection of any subtle changes in shear wave splitting parameters. Scattering can also amplify the P-coda (expressed by the relative increase in amplitudes for the P-coda) and can impede detection of the direct S-arrival, thus making the estimation of the S-arrival, needed for SWS measurements, challenging.

Scattering mechanisms can be grouped into three classes: reflection of seismic waves in a uniformly layered structure, scattering of seismic waves from localized volume heterogeneities, and scattering of seismic waves from irregularities in the boundaries of an otherwise generally layered structure (Sato et al., 2012). For our analysis on the effect of scatterers we will focus on scattering caused by localised volume heterogeneities. Sato et al. (2012) and Shearer (2015) give a concise review of the seismic scattering processes in the crust and deeper earth. 
The particle motion recorded at the Earth's surface does not always represent the particle motion of an incoming shear wave (Nuttli, 1961; Evans, 1984; Liu and Crampin, 1990; Crampin, 1981, 1985; Crampin and Booth, 1989; Crampin and Gao, 2006). This is due to the interaction of the incoming wave with the free surface, which can lead to body-wave phase conversion. The measured particle motion can adopt an elliptical shape even if traveling in an isotropic medium (Nuttli, 1961; Liu and Crampin, 1990; Crampin, 1985; Crampin and Booth, 1989; Crampin and Gao, 2006). This leads to apparent shear wave splitting with the apparent fast direction aligned along the direction of the incoming wave (Liu and Crampin, 1990; Crampin, 1985; Crampin and Booth, 1989). There have been suggestions that shear waves with high incidence angles often show these distortions, with the particle motions becoming non-linear. Conversely, those with a smaller incidence angle (propagating near vertically) often show smaller distortions, with particle motions being linear in an isotropic medium (Nuttli, 1961; Liu and Crampin, 1990; Crampin, 1985; Crampin and Booth, 1989; Crampin and Gao, 2006). Crampin and Booth (1989) and Liu and Crampin (1990) introduced the term "shear wave window", which describes a window of incidence angles within which recorded shear waves are less distorted and are useful for SWS analysis. Neuberg and Pointer (2000) showed that waveforms outside the "shear wave window" generate elliptical particle motion even without the presence of anisotropy, especially when recording shallow local earthquakes in the vicinity of strong topography. Crampin (1990b) and Liu and Crampin (1990) suggested that the effect of shear-waves scattered at the free surface can be minimized when shear waves with incidence angles within the shear wave window are used. They observed that when the angle of incidence is less than the critical angle, $i_{c}=\sin ^{-1}\left(V_{s} / V_{p}\right)$, the reflection coefficients are real, there are fewer phase conversions, and the linear polarization of the incident wave is essentially retained at the surface. Thus waveforms of shear-waves recorded at the surface are similar to the incident waveforms only when the angle of incidence lies within the shear-wave window (Liu and Crampin, 1990; Crampin, 1990b, 1985; Crampin and Booth, 1989; Crampin and Gao, 2006).

Focal mechanisms and spectral source parameters of an earthquakes are often used 
to characterize the source properties of an earthquake. The parameters of the focal mechanism (strike, dip and rake) and the receiver back-azimuth and take-off angles determine the polarization of the incoming shear wave at each receiver (Aki and Richards, 2002). Thus different source properties will yield different polarizations of the incoming shear wave. In shear wave splitting, the polarization of the incoming shear wave can be parallel or perpendicular to an axis of symmetry which can yield a null measurement. Null measurements can introduce significant instability and uncertainty in SWS measurements. We test this by varying the earthquake fault orientations.

\subsection{Method}

In this Chapter we generate synthetic earthquakes in a range of scenarios, and generate synthetic seismograms at an array of locations in order to demonstrate the effect of incidence angle, source mechanism and scatterers. Our synthetic seismograms are computed using the spectral element method, SEM, which is implemented in the SpecFEM3D package (Komatitsch and Tromp, 1999; Komatitsch et al., 2000; Komatitsch and Tromp, 2002) and the 1-D reflectivity code by Levin and Park (1997), which has subsequently been modified by Castellazzi et al. (2015) and Walsh (2012). In recent ears the SEM has been shown to be a highly accurate technique to simulate seismic wave propagation in both isotropic and anisotropic media (Komatitsch et al., 2000; Komatitsch and Tromp, 2002; Chevrot et al., 2004; Chen and Tromp, 2007). A justification for choosing the SpecFEM3D package and a brief overview of the SpecFEM3D method is presented in Section 2.2.3 of the methods Chapter. In the following section, we mainly discuss how we implemented SpecFEM3D for the analysis. For description of terms used here, see Section 2.2.3.

\subsubsection{1-D modeling}

We use the 1-D modeling program, anirec, to simulate synthetic seismograms, using the reflectivity technique, based on a specified velocity model. The seismogram is computed based on the reflectivity algorithm of Chen (1993) and the propagation matrix 
based the on Keith and Crampin (1977) algorithm. In the reflectivity technique the transmission response of a flat-layered medium with an arbitrary oriented hexagonally symmetric anisotropy is computed (Levin and Park, 1997). A sub-vertically travelling wave is assumed to propagate upwards from the half-space into the layered part of the model, where it undergoes refraction, reflection and conversion. The pulses that arrive at the free surface are combined to form the transmission response of the medium. This transmission response is then convolved with pulse of an assumed incident wave to generate the synthetic seismogram (Levin and Park, 1997).

The method implemented in anirec requires numerous parameters such as the velocity model, phase velocity, back azimuth, wave type, plot duration (start time and end time of the seismogram). To gain intuition into the basic physics underlying the representation of the elastic stress-strain relationship, a hexagonal axis of symmetry is assumed (Levin and Park, 1997). The hexagonal axis of symmetry is described by the orientation angles describing the axis of symmetry and the elastic parameters. We limit the tensor in our model to seven parameters; five elastic parameters, $A, B, C, D$ and $E$, and two angles (orientation angles describing the axis of symmetry). Levin and Park (1997), interpreted the fractional values of $A, C, E$ as peak-to-peak relative velocity percentages. For each layer of the medium, we prescribe values of seismic velocities and hexagonally symmetric anisotropy (anisotropic parameters) about a common symmetry axis of a given orientation. For a horizontal axis of symmetry, the azimuthal dependence of the P-wave and S-wave (both vertical and horizontal) velocities can be approximated to first order in terms of the orientation of the symmetry plane in Equation 5.1 (Levin and Park, 1997; Shearer and Orcutt, 1986; Backus, 1965).

$$
\begin{aligned}
& V_{p}^{2}=A+B_{c} \cos 2 \varphi+B_{s} \sin 2 \varphi+C_{c} \cos 4 \varphi+C_{s} \sin 4 \varphi \\
& V_{s v}^{2}=D+E_{c} \cos 4 \varphi+E_{c} \sin 4 \varphi \\
& V_{s h}^{2}=F+G_{c} \cos 2 \varphi+G_{s} \cos 2 \varphi
\end{aligned}
$$

where $V_{p}$ is the $\mathrm{P}$-wave velocity, $V_{s v}$ and $V_{s h}$ are the $\mathrm{S}$-wave (both vertical and horizontal) velocity, and $\varphi$ is the angle describing the azimuth of the axis of symmetry 
(Backus, 1965; Shearer and Orcutt, 1986). The relationship between the coefficients and elastic tensors are shown below:

$$
\begin{aligned}
& A=\frac{3}{8}\left(C_{1111}+C_{2222}\right)+\frac{1}{4}\left(C_{1122}+C_{1212}\right) \\
& B_{c}=\frac{1}{2}\left(C_{1111}+C_{2222}\right), B_{s}=\left(C_{2111}+C_{1222}\right) \\
& C_{c}=\frac{1}{8}\left(C_{1111}+C_{2222}\right)+\frac{1}{4}\left(C_{1122}+C_{1212}\right) \\
& C_{s}=\frac{1}{2}\left(C_{2111}+C_{1222}\right) \\
& D=\frac{1}{8}\left(C_{1111}+C_{2222}\right)+\frac{1}{4}\left(C_{1122}+2 C_{1212}\right) \\
& E_{c}=-C_{c}, E_{s}=-C_{s} \\
& F=\frac{1}{2}\left(C_{1313}+C_{2323}\right) \\
& G_{c}=\frac{1}{2}\left(C_{1313}+C_{2323}\right), G_{s}=C_{2313}
\end{aligned}
$$

where A, B, C, D and E are constants that are related to components of the anisotropic elastics tensors (Backus, 1965; Shearer and Orcutt, 1986). The $c$ and $s$ indexes represent the cosine and sine terms.

\subsubsection{Mesh design for SpecFEM3D}

One of the critical requirements for the spectral-element simulation is a high-quality mesh relevant for the 3D model of interest. The process of constructing an appropriate 3D model requires several processing parameters and constraints such as:

1. the number of grid points per the shortest desired wavelength

2. a balance between numerical cost and available computing resources

3. the numerical stability condition and the acceptable distortion of the elements.

The spectral element method (SEM), which is implemented in SpecFEM3D, requires the computation model (mesh) to be composed of non-overlapping elements. At each element, the wave-field is discretized in terms of high-degree Lagrange polynomials and computed on the Gauss-Lobatto-Legendre (GLL) points which improves numerical accuracy and efficiency (Komatitsch and Tromp, 1999). To implement this, we require each element of the mesh to contain $(n+1)^{3}$ GLL points (where $n$ is the number 
of polynomials). For this study, a Lagrange polynomial of degree four (based on the recommendation of Komatitsch and Tromp, 1999) is used to sample the wave field. Thus each spectral element contains $(4+1)^{3}=125$ GLL points (5 GLL points per wavelength). This approach requires that the mesh elements are hexahedral elements (Komatitsch and Tromp, 1999). We used Cubit v 16.4 (www.csimsoft.com/trelis.jsp) in conjunction with GeoCubit (a Python based script; Casarotti et al., 2008b) to generate the mesh.

The physical domain of the model is approximately $100 \mathrm{~km}$ by $100 \mathrm{~km}$ at the surface, and extends to a depth of $50 \mathrm{~km}$ with an element size of $1 \mathrm{~km}$. Based on the element size and the GLL points used, our simulated waveforms are numerically accurate down to a period of $0.7 \mathrm{~s}$ (or up to $1.33 \mathrm{~Hz}$ ). Based on the resolved period and the wave speeds used (4.310 and $2.490 \mathrm{~km} / \mathrm{s}$ for P- and S-wave respectively), the averaged wavelength of our simulated waveforms is $2.38 \mathrm{~km}$. We sample the wavefield every $0.0015 \mathrm{~s}$ for 20,000 time steps, resulting in a $30 \mathrm{~s}$ waveform, which is more than enough to capture all the needed body-wave phases for our analysis. The depth of the source used for simulation is $15 \mathrm{~km}$. We designed the source-receiver geometry to be similar to the GeoNet and DPRI seismic network (used in Chapter 3 of this thesis), and additional receivers were designed to address some of the research questions (Figure 5.1). To avoid recording boundary-edge effects, we placed the stations at least $20 \mathrm{~km}$ away from the boundary of the model. Additional absorbing boundary conditions were also applied on all sides of the model in the SpecFEM3D programme. Also, to avoid the influence of boundary reflections from the bottom of the mesh, we extend the mesh to a depth of $50 \mathrm{~km}$ (to ensure there is sufficient distance for the downward propagating waves not to bounce back to contaminate the upward waves).

\subsubsection{Implementation and verification of anisotropic models}

We initially consider a theoretical model of an anisotropic medium filled with cracks. We used the theory of Hudson (1981), which is implemented in the MSAT package (A MATLAB toolkit for the analysis and calculation of elastic tensors; Walker and Wookey, 2012) to calculate the effective medium elastic tensors/constants. The 
$\begin{array}{ll}4 & 0 \\ 0 & 0 \\ 0 & 0 \\ > & 0\end{array}$

के

क

ค ग

宓

身

ฮี ญ

in है

¿

O

규

$\pi N$

尝

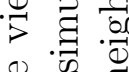

令江

以

ग

ली

< $\bigcup_{0}^{0}$

$\ddot{\theta}$ \&

잉

吾声

. 겅

䦎

分寻

¿ु

ச $\dot{2}$

일.

은

要进

可

๑ 节

Ð

○

Ð

कै के थ

$-00$

$\geq$ 용

¿ 02

車

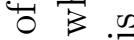

司沗

E 胥

Ð

ㄷ.

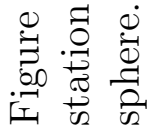


elastic tensors were subsequently incorporated into the seismic modelling package (SpecFEM3D) to conduct the forward modelling of the seismic waves following the approach of Chen and Tromp (2007) and Chevrot et al. (2004). The elastic constants were calculated using density, P- and S-wave velocities of $2670 \mathrm{~kg} / \mathrm{m}^{3}, 5.640 \mathrm{~km} / \mathrm{s}$ and $3.320 \mathrm{~km} / \mathrm{s}$ respectively. The cracks were characterised by the isotropic elastic parameters of the fill material, the crack density and the aspect ratio of the crack (the ratio of the cracks radius to the crack width; Walker and Wookey, 2012). Here we used water filled cracks with $\mathrm{P}$ - and $\mathrm{S}$-wave velocities of $1.5 \mathrm{~km} / \mathrm{s}$ and $0 \mathrm{~km} / \mathrm{s}$ respectively and density of $1000 \mathrm{~kg} / \mathrm{m}^{3}$. We used a crack density of 0.06 with an aspect ratio of $5.26 \times 10^{4}$, which yielded a delay time of $0.21 \mathrm{~s}$ and a fast direction direction of $0^{\circ}$ for our source depth of $15 \mathrm{~km}$ and station X032, which is nearly vertical above the source (the fast direction can be changed by rotating the elasticity matrix/tensor about an azimuth; Hammond et al., 2010; Walker and Wookey, 2012).

To verify that we are incorporating the correct anisotropic tensor into the SpecFEM3D package, we first designed a simple numerical experiment to simulate waveforms that are (1) propagating through the anisotropic medium and (2) propagating through an isotropic medium (used as a reference model). We compared the waveforms from the isotropic and anisotropic model. Figure 5.2 shows an example waveform comparison from a station $\mathrm{X} 032$, which is close to the source at the surface. Compared to the isotropic model, the horizontal components of the waveform from the anisotropic model show clear differences in shear phase arrival times (indicating the slow and fast wave), as expected for waveforms propagating in an anisotropic medium. This arrival time difference of $0.21 \mathrm{~s}$ recorded on station X032 is the expected delay time for the elastic constants and propagation direction used. We also estimated the splitting parameter for the recorded waveform (station X032) using the MFAST code (Wessel, 2010; Savage et al., 2010a) and we retrieved the expected delay time of $0.21 \mathrm{~s}$ and fast direction of $0^{\circ}$. Figure 5.3 shows an example of MFAST diagnostic plots of shear-wave splitting analysis and a polar view representation of the velocities derived from various elasticity tensors used in our simulations.

To further gain confidence in the specification of the elastic tensors used, we rotated 


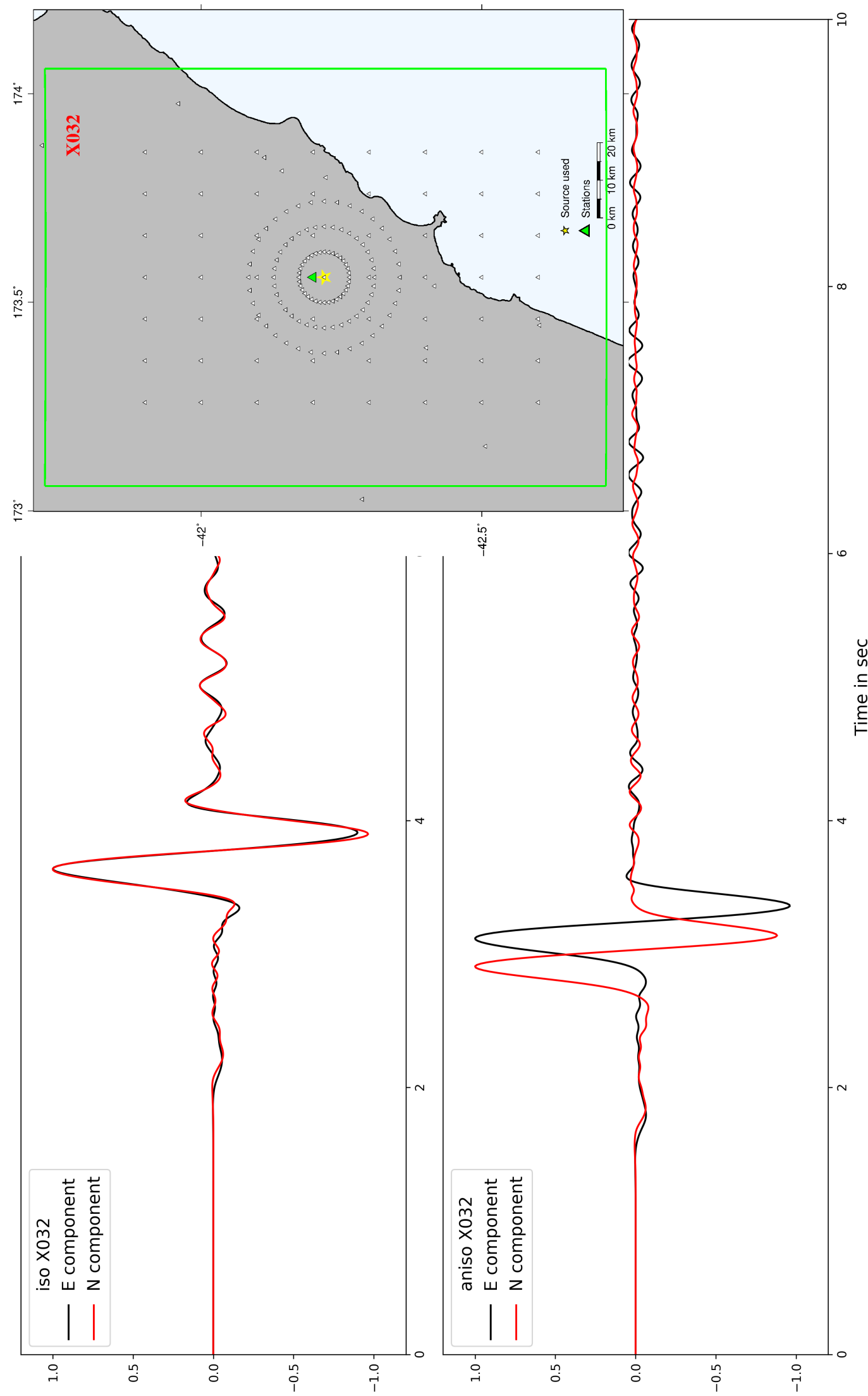

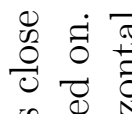

. $\frac{0}{0}$

䕇

규 पू

.

क

द्व

青定寻

萦离

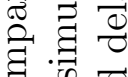

0.

द्व̈

赵

3 का

을

สี

更

ซี के के

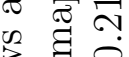

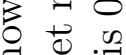

क

의

So $\$$

ज न

Е ๙ิ

हี

$\stackrel{0}{>}$.

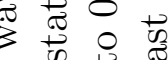

ن 0 舟

范

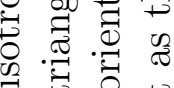

бี

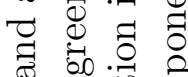

若兽

苛

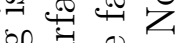

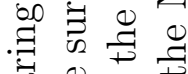

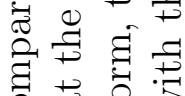

范

ن

is 0

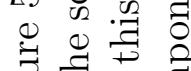

bo 

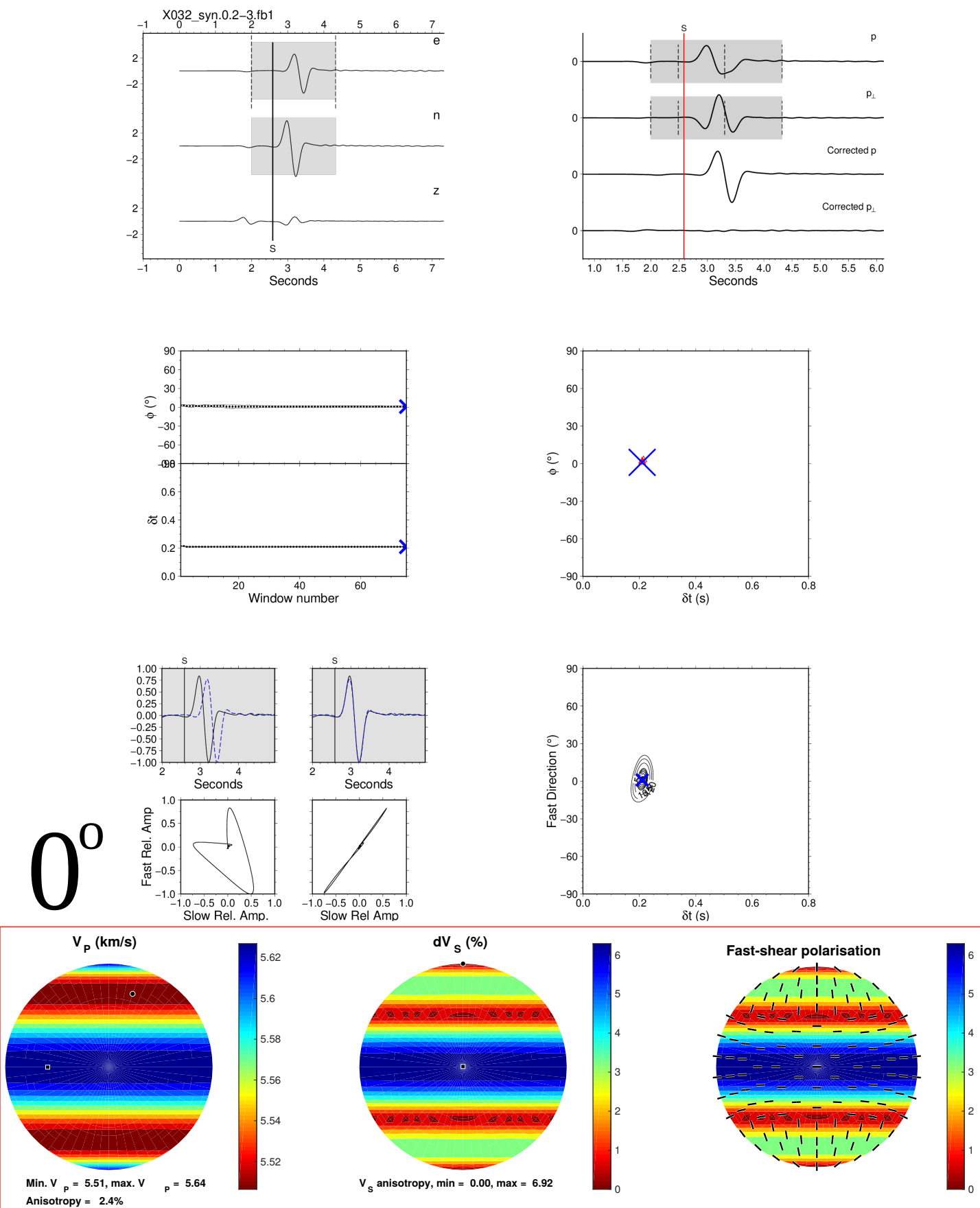

Figure 5.3: The top two panel shows MFAST diagnostic plots of SWS analysis illustrating the procedure used in estimating the splitting parameters (see Figure 2.3 for full description). The bottom panel (red box) is a polar view representation of different elasticity tensors used. It shows an equal area lower hemisphere projection plot of seismic velocities. The magnitude of the slow and fast shear wave velocity and its fast polarization direction (black thick marks) are shown on the left-hand side and middle plots, respectively. The centre of the hemisphere corresponds to vertical wave propagation, and the perimeter of the hemisphere shows azimuthal variations in horizontal velocities. The maximum and minimum $\mathrm{P}$-wave velocity and the $\mathrm{P}$-wave anisotropy are also given below each hemisphere in this column. The description of MSAT output follows that of Walker and Wookey (2012). 
Table 5.1: Elastic tensors used in validating the initial model.

\begin{tabular}{ccccc}
\hline $\mathrm{C}_{i j}$ & no rotation & $20^{\circ}$ rotation & $60^{\circ}$ rotation & $90^{\circ}$ rotation \\
\hline $\mathrm{C}_{11}$ & 84.5145 & 83.0079 & 81.9829 & 84.8923 \\
$\mathrm{C}_{12}$ & 25.9440 & 27.4947 & 28.7589 & 25.9440 \\
$\mathrm{C}_{13}$ & 25.9440 & 25.9544 & 26.0105 & 26.0327 \\
$\mathrm{C}_{16}$ & 0.0000 & -1.7874 & 1.7070 & 0.0000 \\
$\mathrm{C}_{22}$ & 84.8923 & 83.2974 & 81.7940 & 84.5145 \\
$\mathrm{C}_{23}$ & 26.0327 & 26.0223 & 25.9662 & 25.9440 \\
$\mathrm{C}_{26}$ & 0.0000 & 1.9089 & -1.5434 & 0.0000 \\
$\mathrm{C}_{33}$ & 84.8923 & 84.8923 & 84.8923 & 84.8923 \\
$\mathrm{C}_{36}$ & 0.0000 & 0.0285 & 0.0384 & 0.0000 \\
$\mathrm{C}_{44}$ & 29.4298 & 28.9849 & 26.5773 & 25.6264 \\
$\mathrm{C}_{45}$ & 0.0000 & 1.2224 & 1.6469 & 0.0000 \\
$\mathrm{C}_{55}$ & 25.6264 & 26.0713 & 28.4790 & 29.4298 \\
$\mathrm{C}_{66}$ & 25.6264 & 27.1772 & 28.4414 & 25.6264 \\
\hline
\end{tabular}

Elastic parameters $\mathrm{C}_{i j}$ are in $10^{9} \mathrm{~kg} \mathrm{~m} \mathrm{~m}^{-1} \mathrm{~s}^{2}$

the elasticity matrix/tensor by azimuth of $20^{\circ}, 30^{\circ}$ and $90^{\circ}$, and for each rotation we simulated the waveforms and estimated the splitting parameters. Figure C.1 - C.3 of Appendix $\mathrm{C}$ show the polar view representation of the elasticity tensor and an output plot from MFAST for $20^{\circ}, 30^{\circ}$ and $90^{\circ}$ rotation of elasticity matrix about the azimuth respectively. Table 5.1 shows the elastic tensors for each rotation. For each rotation $\left(20^{\circ}, 30^{\circ}\right.$ and $\left.90^{\circ}\right)$, we expected a fast direction of $20^{\circ}, 30^{\circ}$ and $90^{\circ}$ which we do indeed find after running the waveform through MFAST. This gave us confidence in our specification of the elastic tensors used in this study.

\subsubsection{Model selection}

Numerical artifacts can arise as a result of a poor-quality mesh. This may lead to an increase in computational cost or inaccurate results. Often, geological models are constructed as layered models. These layers may serve as discontinuities and produce unreal diffractions. Thus, for an accurate mesh, one should model at least the major geological discontinuities in the model. We tested two models; a two layer model and single layer model. The two layer model had a $10 \mathrm{~km}$ thick anisotropic layer on top of an isotropic homogeneous elastic volume with a thickness of $40 \mathrm{~km}$. For the single layer model we used an anisotropic homogeneous elastic volume with a thickness of $50 \mathrm{~km}$ 
(Figure 5.4). The description of the anisotropic parameter used is presented in Section 5.2.3 (implementation and verification of anisotropic models). We used a right-lateral strike-slip fault as source (EventID “2013p543824” on Table 5.2). We used the elastic tensor labelled "no rotation" from Table 5.1 which yields a fast direction of $0^{\circ}$ and delay time of $0.21 \mathrm{~s}$. Comparing the waveforms (using the same low pass filter of $1.33 \mathrm{~Hz}$ ) for the two models, we observed precursory signals before the shear arrival (shaded window after the $\mathrm{P}$ arrival in Figure 5.5) for the two layer model. The precursory signals are stronger on the north component compared to the other components, which may be P-S or S-P phase conversion or an artifact caused by the discontinuity at the boundary (high impedance contrast) of the two layers. This precursory signal was not present on the single volume's waveform. For this reason we settled on a single volume model for this analysis. Moreover, since we are not interested in fitting our result to an actual observed waveform (earthquake), the single volume model was sufficient for this analysis (for simplicity).

\subsubsection{Source parametrization}

In SpecFEM3D, the earthquake source can be described by either a point source or a finite fault model depending on the size of the model, the earthquake magnitude and the research goal. For this study, we used a point source in a form of CentroidMoment-Tensor, CMT solution, which has source information such as the event depth, magnitude and focal mechanism (describing the source). We used the first-derivative Gaussian source-time function (Ricker wavelet) as the source-time function. The CMT solutions used here are modified from the New Zealand CMT solutions catalog (Ristau, 2008). Table 5.2 shows the sources used to test the effect that earthquake source mechanisms have on SWS measurements. 


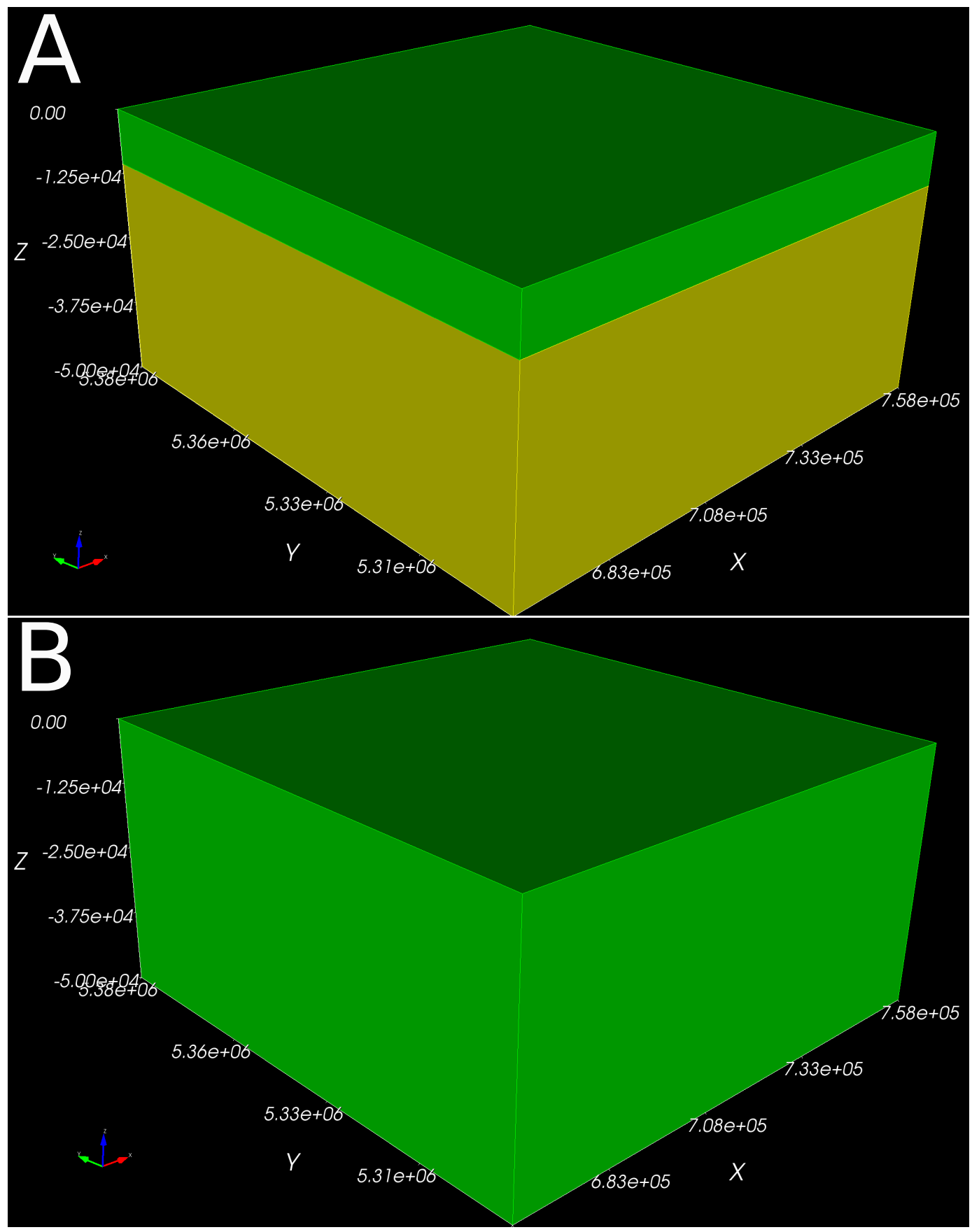

Figure 5.4: Example of initial testing model. (A) A two layer model layer model: a 10 $\mathrm{km}$ thick anisotropic layer directly above an isotropic homogeneous elastic volume with a thickness of $40 \mathrm{~km}$ (B) a single layer of volume model; an anisotropic homogeneous elastic volume with a thickness of $50 \mathrm{~km}$. 


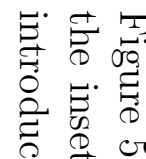

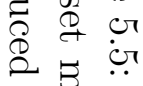

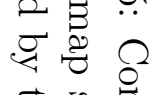

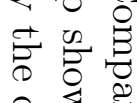

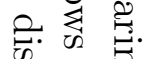

ᄋे

丞.

艺. 节. 官

\& 8

它官宽

里导 宫

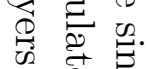

$\rightarrow \stackrel{0}{\circ}$

巴幽

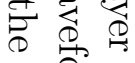

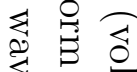

过芯

औह

范

$\begin{array}{lll}2 & 0 & 0 \\ 0 & 0 & 0 \\ 0 & 0 & 0 \\ 0 & 0 & 0\end{array}$

当乞 尺

बै

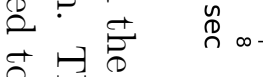

Velocity in $\mathrm{m} / \mathrm{s}$

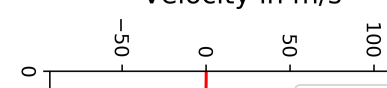

Velocity in $\mathrm{m} / \mathrm{s}$

Velocity in $\mathrm{m} / \mathrm{s}$
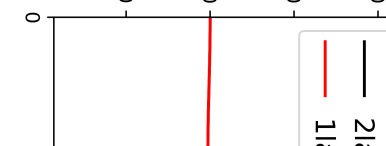

0

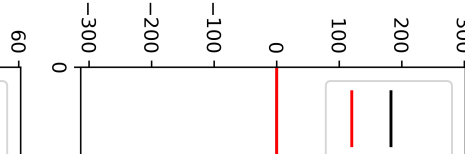

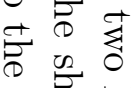

की ڤैं

क

\& 3 .

止? ?

苞官

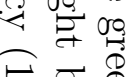

Ðْ

击苛

迹

क्व

(c)

ㄹ.

焉

몽. 뭉

के

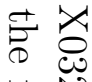

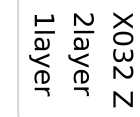
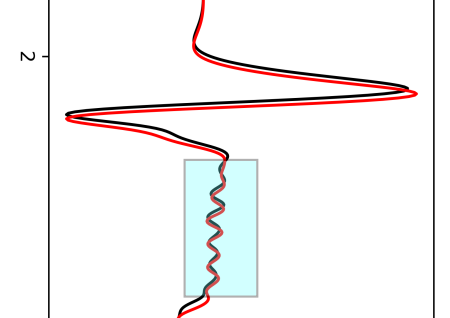

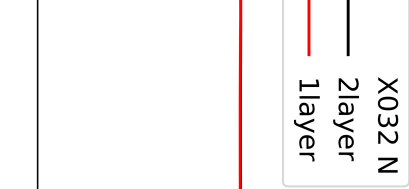

-1.

8
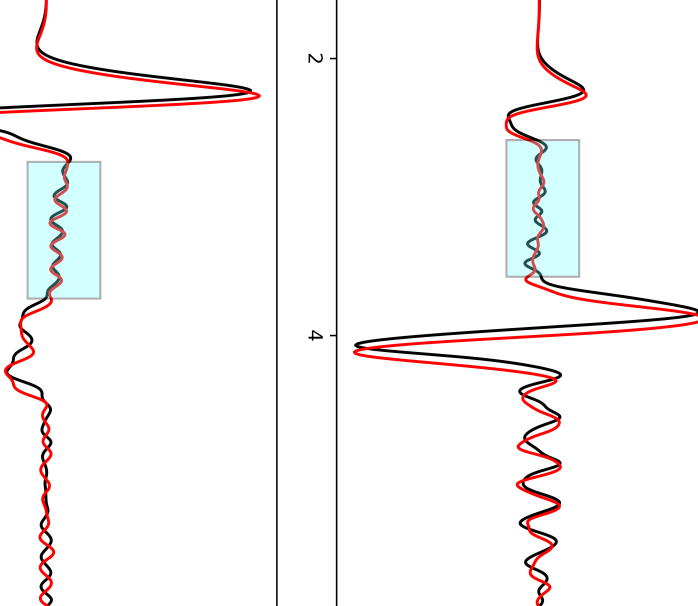


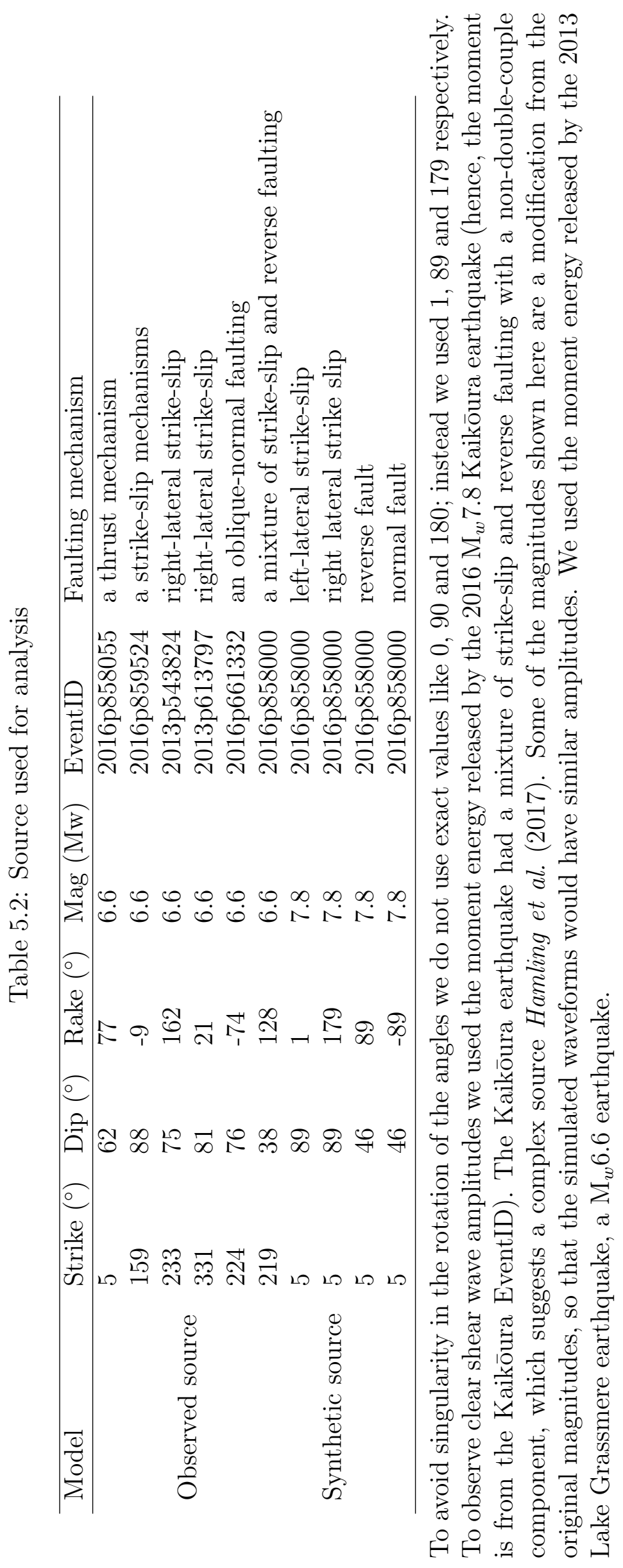




\subsubsection{Models for testing the effect of incidence angle}

Here we used a 1-D synthetic analysis primary to determine the effect that varying the angle of incidence has on splitting measurements. We also confirmed those models with an additional 3D synthetic analysis. This aids in resolving the ultimate critical angle needed for our SWS analysis. This is necessary because as discussed earlier, outside the shear wave window, the shear waves are susceptible to $\mathrm{S}$ to $\mathrm{P}$ conversions and scattering at the surface causing non-linear particle motion (Nuttli, 1961; Crampin and Gao, 2006).

We simulated waveforms in a 1-D anisotropic medium using the Levin and Park (1997) 1-D reflectivity code (which was subsequently modified by Castellazzi et al. (2015) and Walsh (2012)) and estimated the apparent splitting parameters using MFAST (Wessel, 2010; Savage et al., 2010a). Figure 5.6 shows the 1-D model used for analysis. The effect of the angle of incidence on splitting analysis was tested by varying the angle of incidence and back azimuth. For the 3D studies, we simulate seismic wave propagation throughout an anisotropic medium. The stations are positioned in a circular form and separated by $\sim 5 \mathrm{~km}$ distance to sample different back azimuths and different incidence angles. Figure 5.7 illustrates the station configuration used for the $3 \mathrm{D}$ studies. 

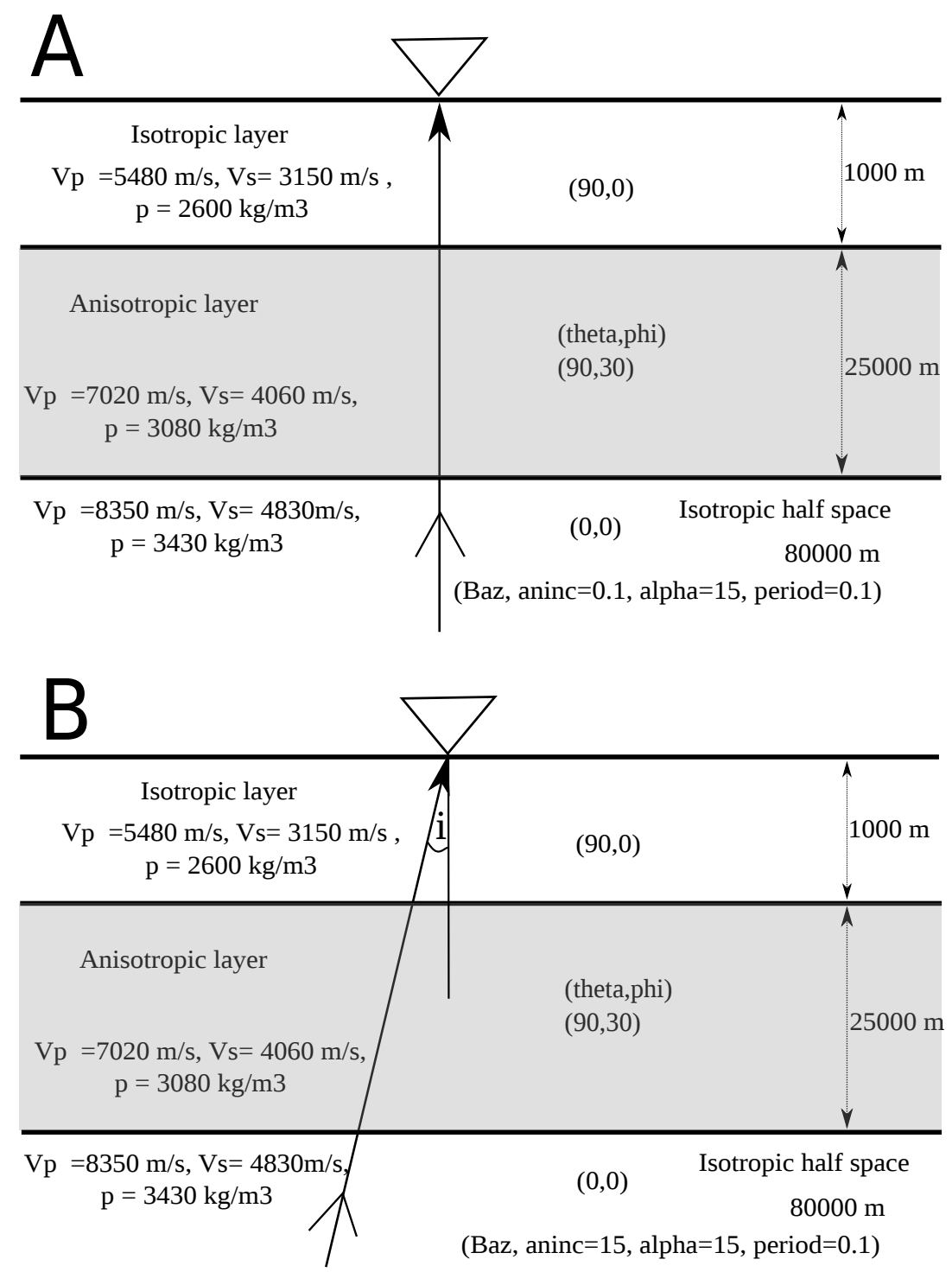

Figure 5.6: 1-D models used in the analysis. The model consists of an anisotropic layer atop isotropic layers. $\mathrm{p}=$ density, $\mathrm{V}_{\mathrm{p}}$ and $\mathrm{V}_{\mathrm{s}}$ are $\mathrm{P}$ and $\mathrm{S}$ velocity respectively. Theta and phi defines the axis of symmetry and $\mathrm{i}$ is the incidence angle. Model A is the baseline model. In model $\mathrm{B}$, we test the effect of incidence angle on splitting results. We used 1-D reflectivity codes (anirec; Levin and Park, 1997) for the waveform simulation in 1-D anisotropic media. 


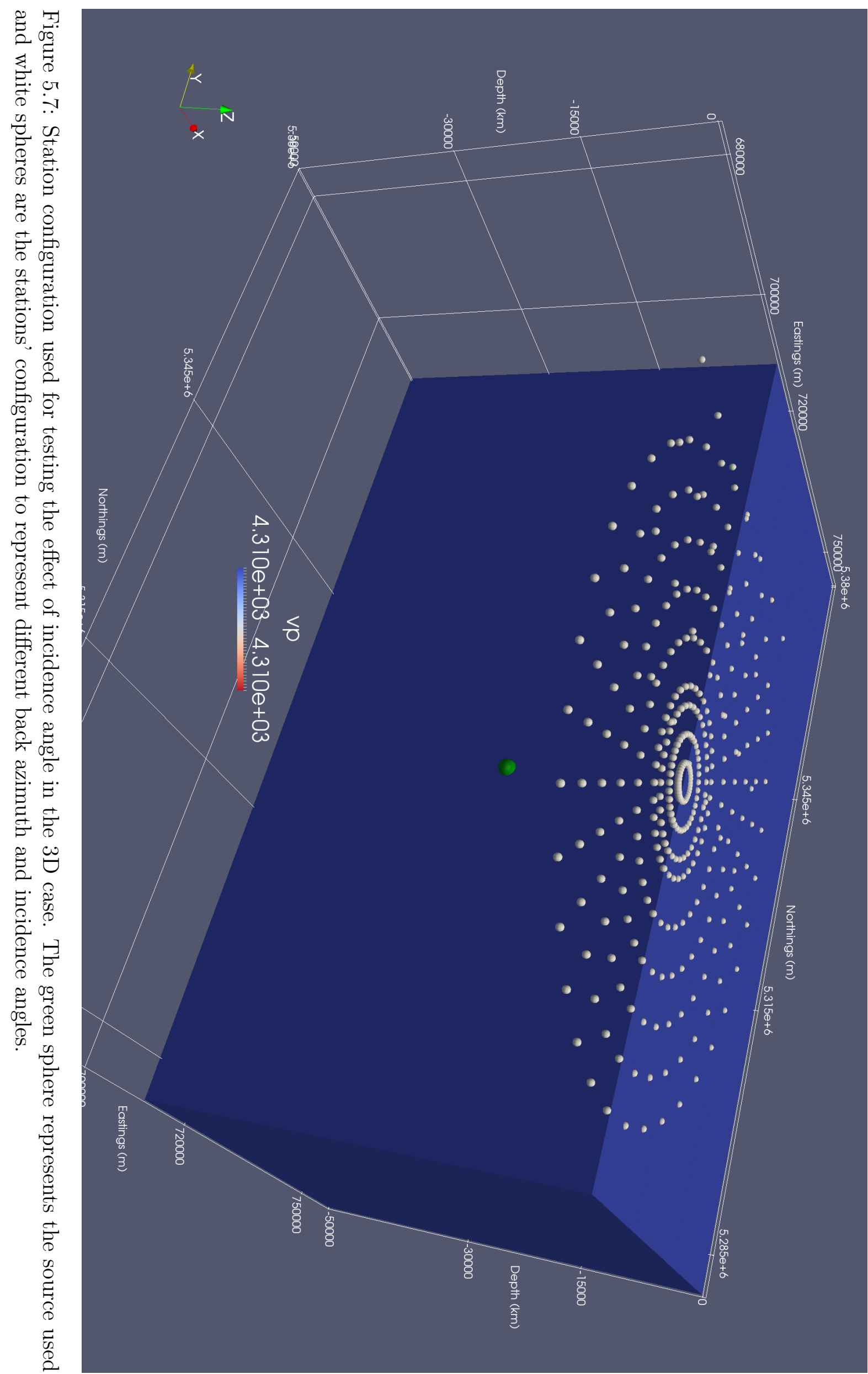




\subsection{Results and Discussion}

\subsubsection{Effect of incidence angle of the incoming shear wave on SWS}

In the $1-\mathrm{D}$ case, we observe a strong back azimuthal variation of $\delta t$ and $\phi$ as the angle of incidence is increased (here vertical incidence is $0^{\circ}$ ) (As shown in Figure 5.8). At a higher angle of incidence $>30^{\circ}$, we observe significant variation in the estimated splitting parameters with back azimuth. This is because there is a real variation of $\phi$ and $\delta t$ with angle of incidence (see Figure 5.3 and and 5.9) and also because phase conversion, at high angles, may interfere with SWS measurements (Nuttli, 1961; Liu and Crampin, 1990; Crampin, 1985). Similar strong back azimuthal variation of $\delta t$ and $\phi$ as the angle of incidence increases is observed in the 3D studies (Figure 5.10). This observation can also be seen on MSAT outputs (e.g. Figure 5.3 and 5.9). Figure 5.9 shows the ring stations used (in the $3 \mathrm{D}$ studies) on the polar view representation of the elasticity tensor used (Walker and Wookey, 2012). The wider the ring, the higher the incidence angle and as the incidence angle increases the delay time and the fast direction also differ. This is clearly seen in the estimates SWS parameters in Figure 5.8 and 5.10. Since we observed only minor variation in splitting parameters with back azimuth at an incidence angle of $35^{\circ}$ compared to a higher incidence angle, we selected a maximum angle of $35^{\circ}$ as the best shear wave window for our analysis.

Often an over simplified straight line ray from source to receiver is used to estimate the angle of incidence, which may affect the estimated values. In reality, lower velocity layers at the surface will likely curve the wave-front and effectively extend the shear wave window by several degrees (Gledhill, 1991; Crampin and Gao, 2006). Thus using a good velocity model to estimate the right incidence angle is essential. For our analysis in the observational studies (Chapter 3 and Chapter 4), we estimated angles of incidence using the TauP toolkit (Crotwell et al., 1999) and a 1-D velocity of the region of interest. 

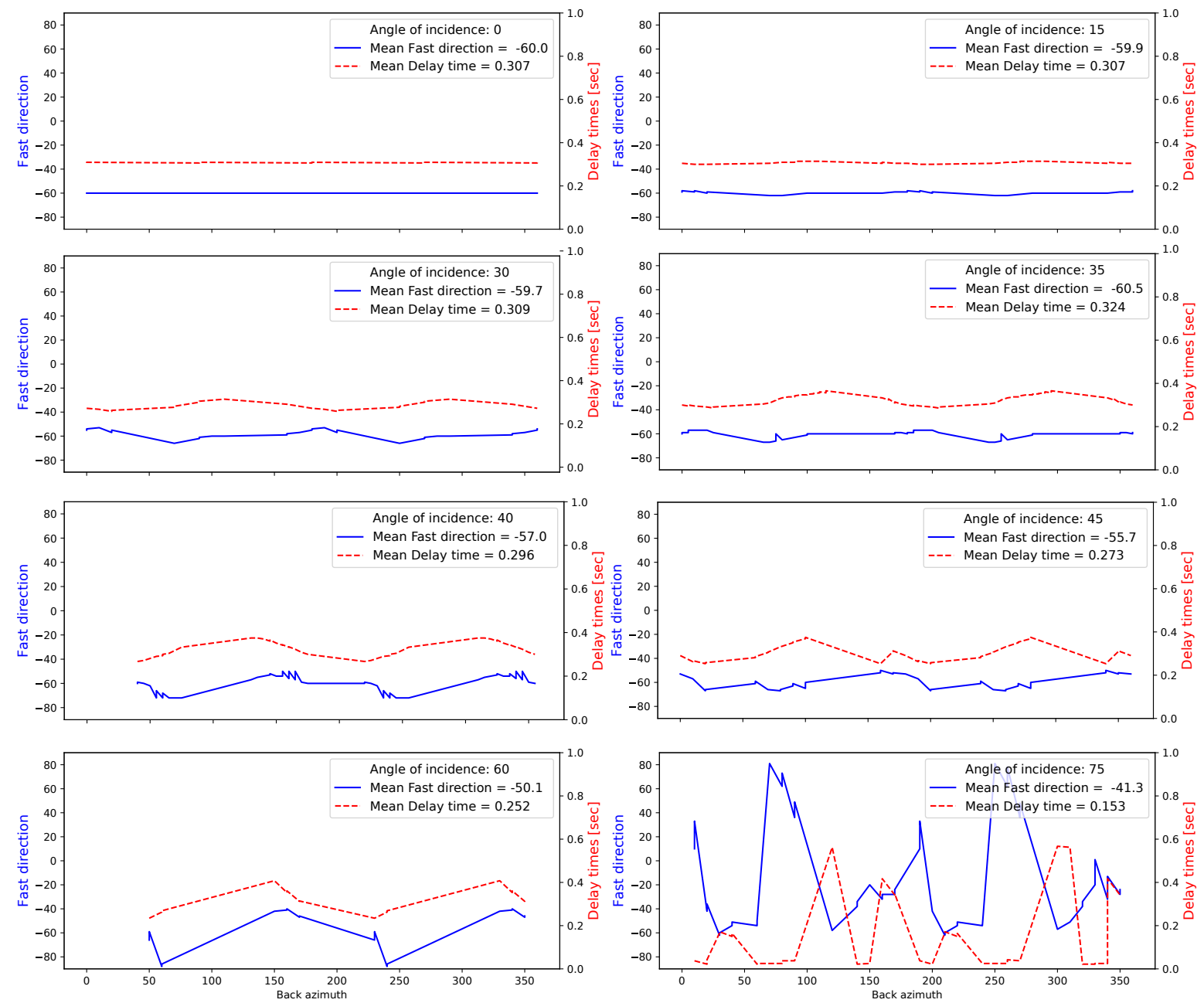

Figure 5.8: Comparison of apparent splitting parameters as a function of back-azimuth ranging from $0^{\circ}$ to $360^{\circ}$ with $5^{\circ}$ increments for the 1-D case. As angle of incidence is increased, there is more variation in $\delta t$ and $\phi$ with varying back-azimuth. This is because there is a real variation if $\phi$ and $\delta t$ with angle of incidence (see Figure 5.3 and and 5.9) and also because phase conversion, at high angles, may interfere with SWS measurements. 


\section{Fast-shear polarisation}
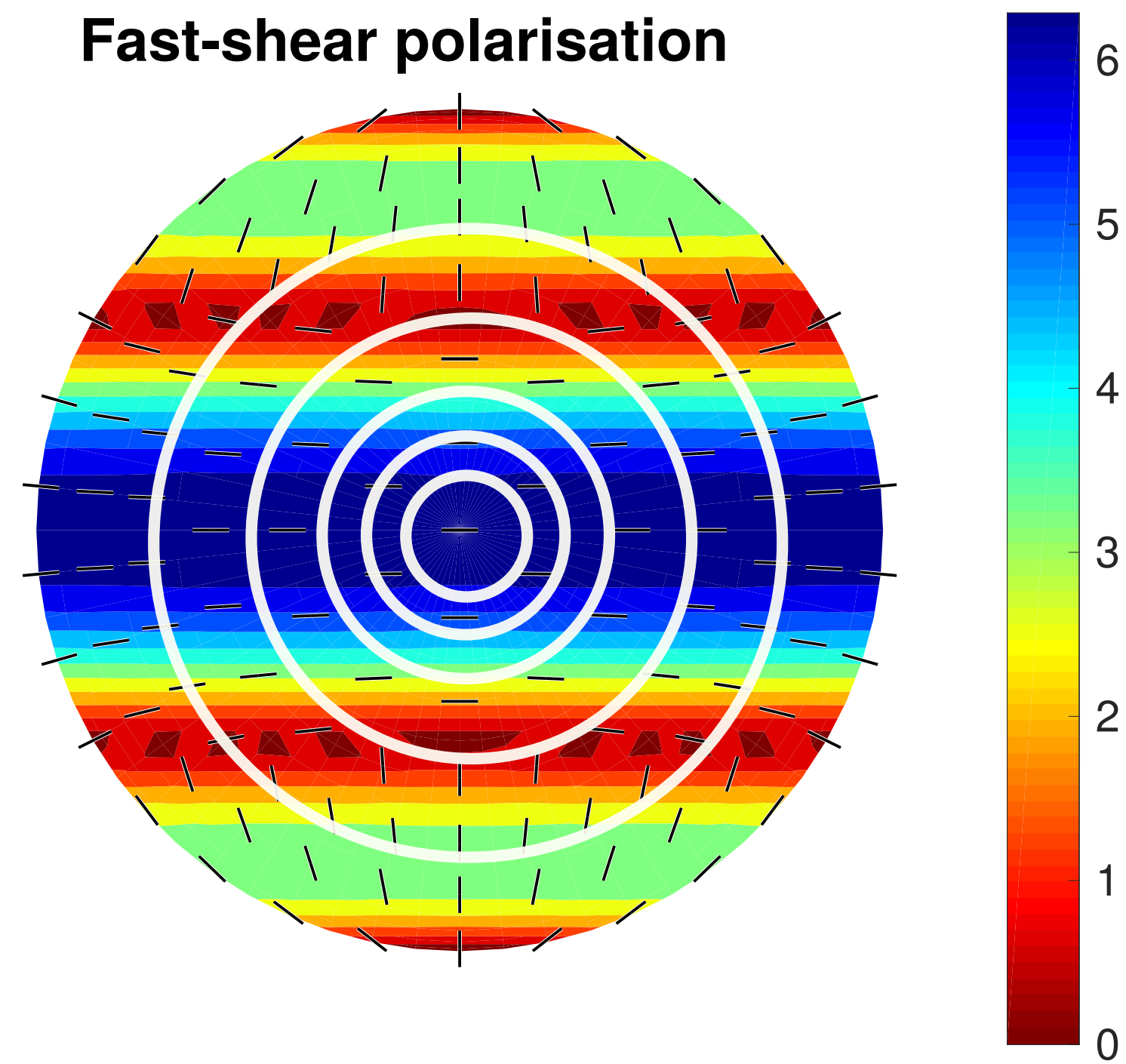

Figure 5.9: The ring stations used are represented as thick white rings on the polar view representation of the elasticity tensor used. The bigger the ring, the higher the incidence angle. The delay time and its fast direction are shown as the background colour and black thick marks respectively. The inner ring represents stations directly above our source and the others represent stations as you move away from the source. 

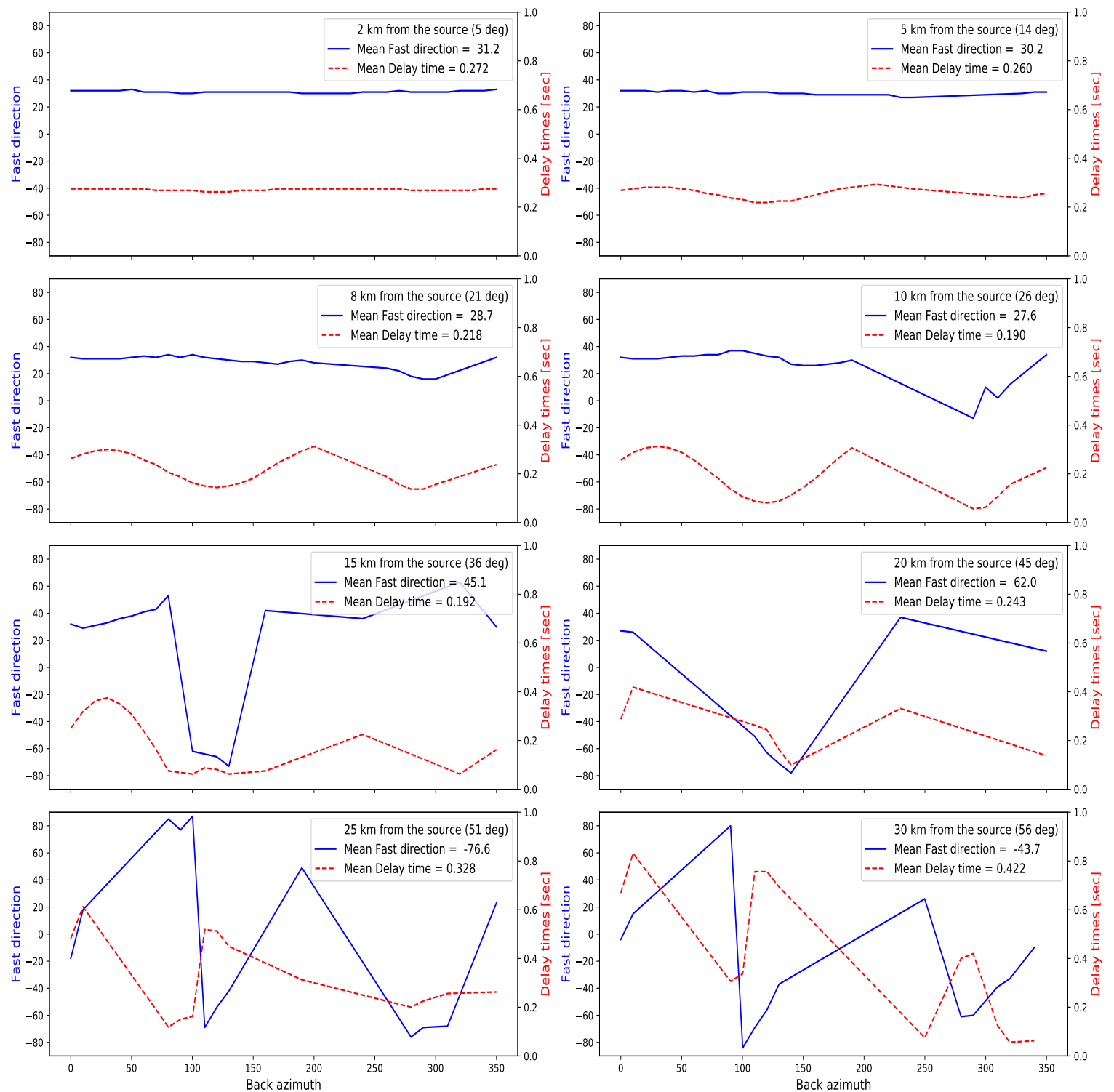

Figure 5.10: Comparison of apparent splitting parameters as a function of back-azimuth ranging from $0^{\circ}$ to $360^{\circ}$ for the $3 \mathrm{D}$ case. As angle of incidence is increased (further from the source), there is more variation in $\delta t$ and $\phi$ with varying back-azimuth. 


\subsubsection{Effect of varying source mechanism}

Here we look at the effect of incoming polarization on the SWS parameters by varying the source mechanism. All variables in the model used to test the effect of incidence angle are held constant except the source mechanism. For the source mechanism we used simple source mechanisms of strike slip, normal and reverse fault mechanisms to estimate the CMT solutions. We also use source mechanisms from observed earthquakes (such as the 2013 Cook Strait and 2016 Kaikōura earthquakes in New Zealand) except that we set the magnitude to be identical so that the amplitudes would be comparable. Table 5.2 shows the different source mechanism parameters used.

Form Figure 5.11 we observe that the effect of source mechanism is not noticeable except at certain back azimuths. The estimated splitting parameters at these back azimuths show sharp divergence from neighbouring estimates (Figure 5.11). At these back azimuths the incoming polarization direction of the shear wave is aligned either with the fast or slow direction. These measurements are "null" results where no splitting can occur, and so the parameters cannot be estimated properly (see Section 2.2.1; Wessel, 2010; Savage et al., 2010a; Balfour et al., 2012). MFAST diagnostic plots from these back azimuths confirm that these are clear null results. An example of the MFAST diagnostic plots for a back azimuth, with a null result is shown in Figure 5.12. 


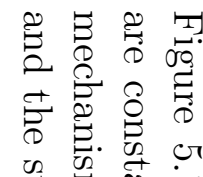

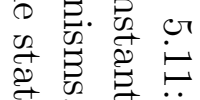

官

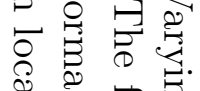

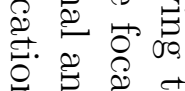

ใ

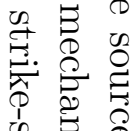

in.

ज苛范

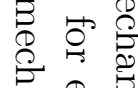

芯. 己ّ

B क ?

范艺

$\stackrel{8}{\Im} \cdot$

它芯

$\rightarrow$ 贾边

。े

$\widetilde{\sigma^{2}}$

है ڤँ

Fast direction

Fast direction

Fast direction

Fast direction

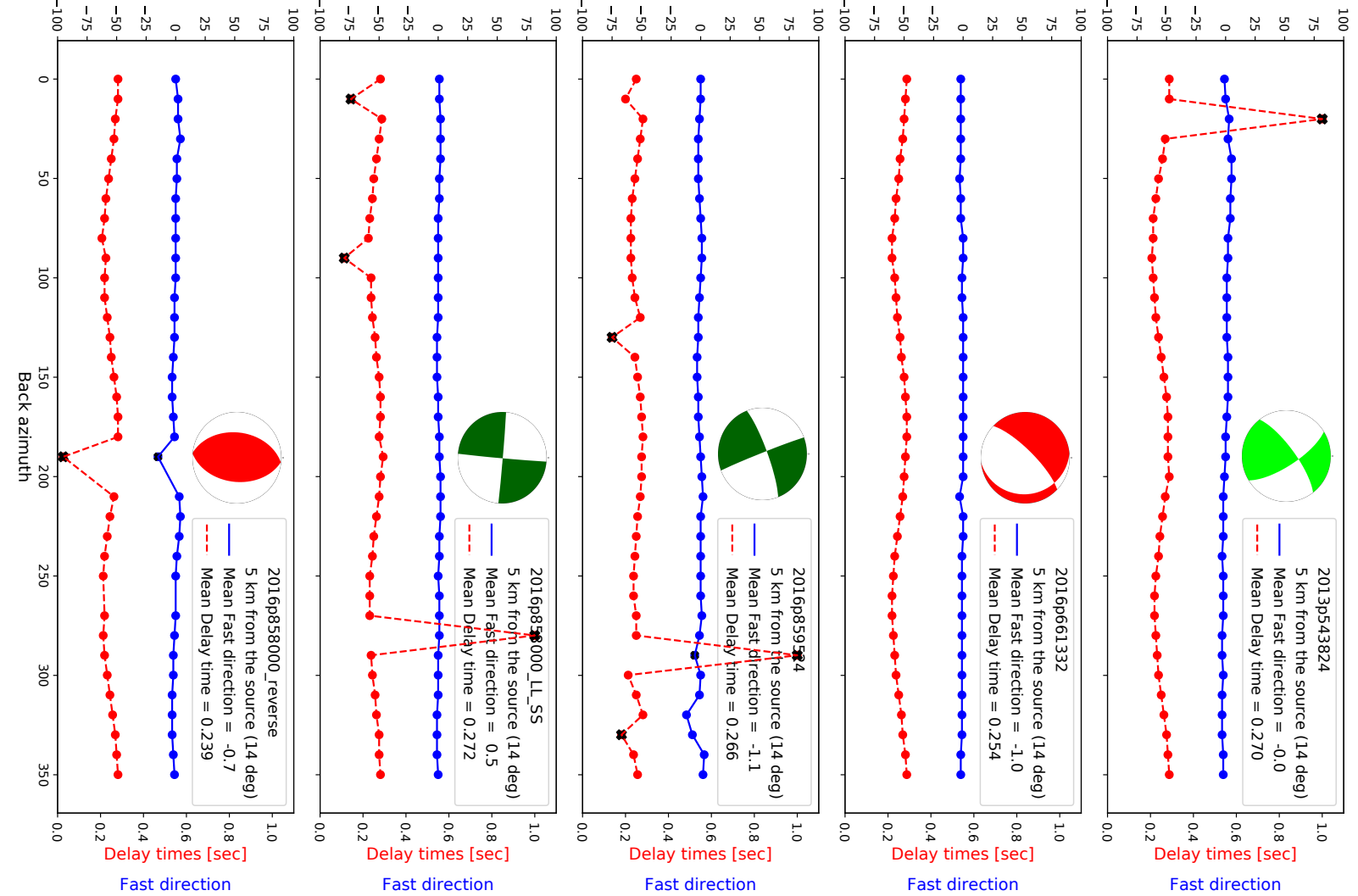

률 己

¿

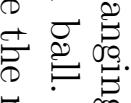

当宫

㿣

$+8$

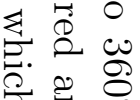

in.

요요

电早

$8 \stackrel{8}{8}$

节官

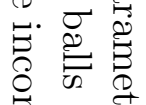

芯. 它

我

"ृ

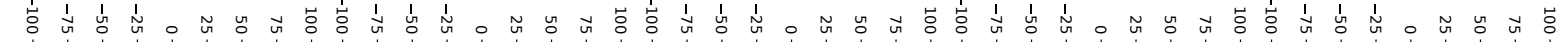
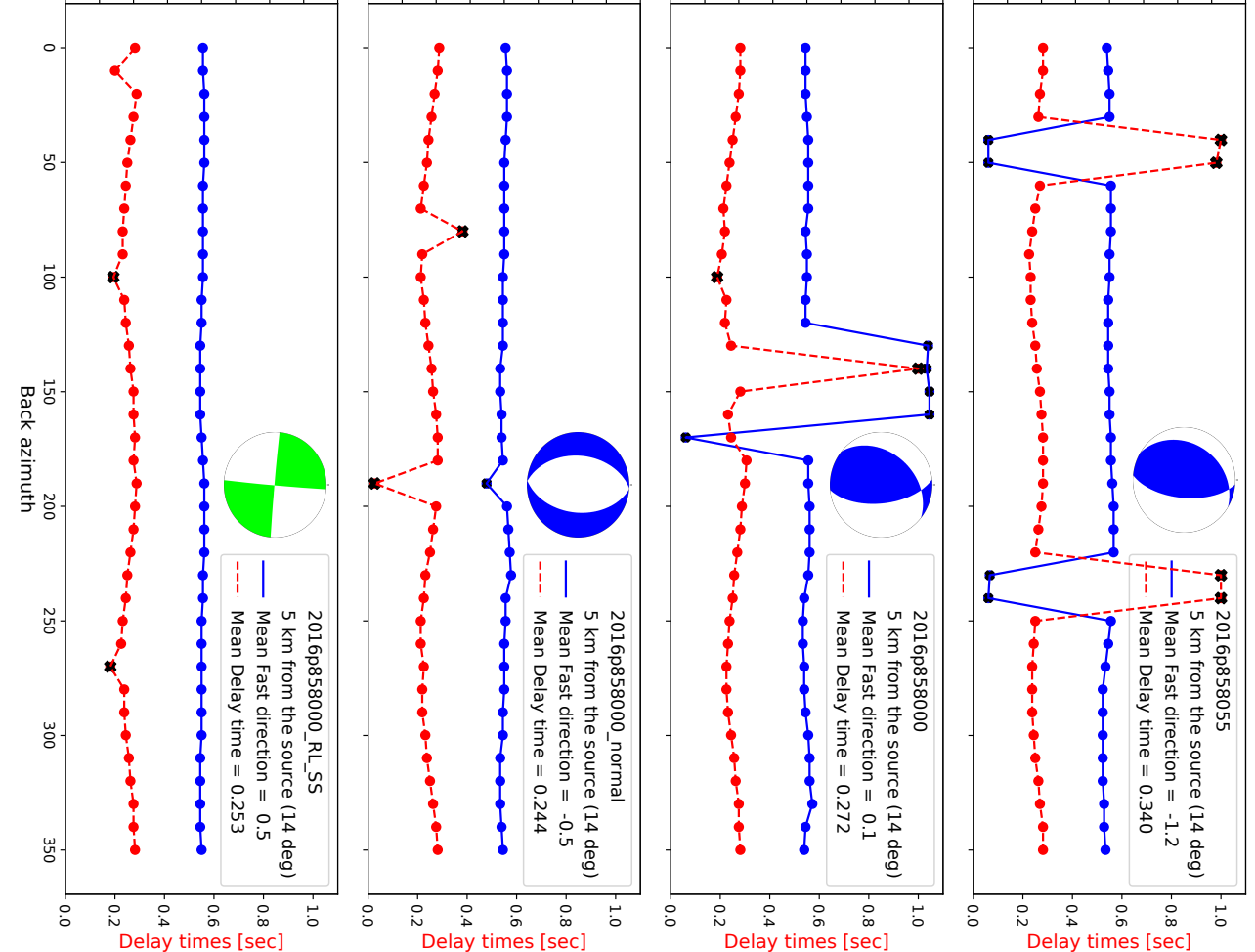

․

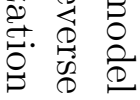



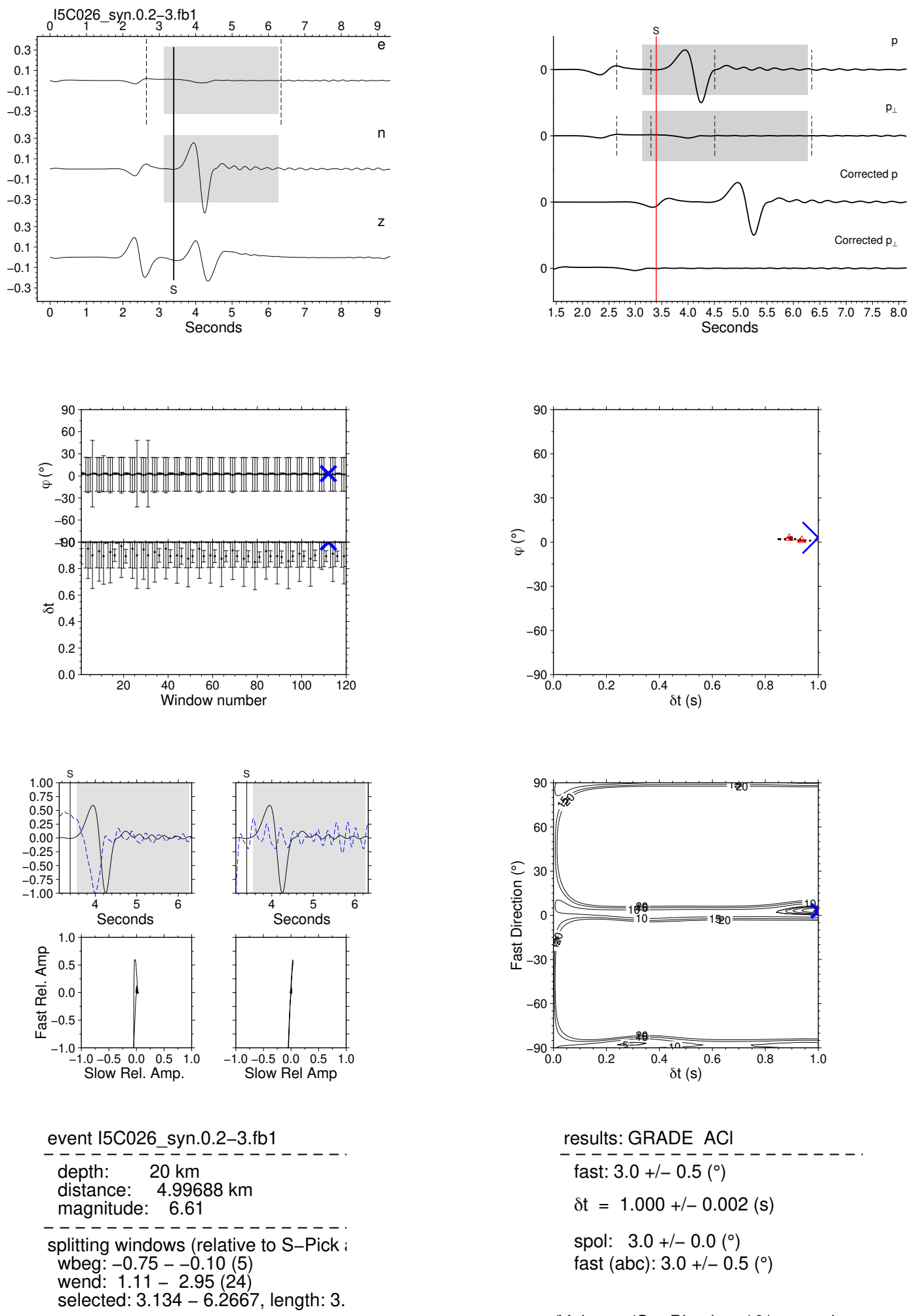

$$
\begin{aligned}
& \text { results: GRADE } \mathrm{ACI} \\
& -\mathrm{fast}: 3.0+/-0.5\left(^{\circ}\right) \\
& \delta \mathrm{t}=1.000+/-0.002(\mathrm{~s}) \\
& \text { spol: } 3.0+/-0.0\left(^{\circ}\right) \\
& \text { fast (abc): } 3.0+/-0.5\left(^{\circ}\right) \\
& \text { Nolumes/GeoPhysics_16/users-data }
\end{aligned}
$$

Figure 5.12: Example of MFAST diagnostic plots for source 2013p543824 at station C026 (located $5 \mathrm{~km}$ away from the source at the surface) showing null results. This figure has the same description as in Figure 2.3 


\subsubsection{Effect of scatterers on SWS measurements}

Here we consider a localised heterogeneous medium as the scatterer. We used both a low velocity anomaly (a proxy for near-surface weathering layers with low body-wave velocities) and a high velocity anomaly (representing a cooled volcanic rock intrusion). An illustration of the introduced localized anomaly is shown in Figure 5.13. We position stations to record waveforms that are: (1) propagating through the anomalous medium (2) propagating at the edge on the anomalous medium and (3) not going through the anomalous medium. Figure 5.14 illustrates a simplified source receiver configuration used to test the effect of scatterers on SWS measurements. All variables in the model used to test the effect of incidence angle are held constant except for the introduced anomalous body. We also tested a model with no anomalous body to serve as the reference model. We compare waveforms and SWS measurements from the inhomogeneous model to the reference model.

Figure 5.15, 5.16 and 5.17 show waveforms that are; (1) not going through the high velocity anomalous medium (scatterer), (2) propagating through the high velocity anomalous medium and (3) propagating at the edge on the anomalous medium respectively. Waveforms recorded on stations far away from the scatterer (green circle in Figure 5.14) show little difference from waveforms from the reference model (Figure 5.15). This is expected since the wave did not encounter much of the heterogeneity. For a waveform recorded at the station directly above on the scatterer (black circle in Figure 5.14), we observe a time lag between the waveform from the reference model and the inhomogeneous model (Figure 5.16). The faster arrival on the phases of the waveform from the inhomogeneous model is an indication that the waveforms was propagating through a high velocity anomalous medium. The vertical component also shows a reflected phase arriving soon after the $\mathrm{S}$ arrival. Waveform distortion is clearly observed for the waveform recorded on the station (blue circle in Figure 5.14) at the edge the anomalous medium (Figure 5.17). This observation is likely due to the interaction of the primary wave (thick ray-path to blue station from Figure 5.14) with the secondary wave (broken line with ray path going through the scatterer from Figure 

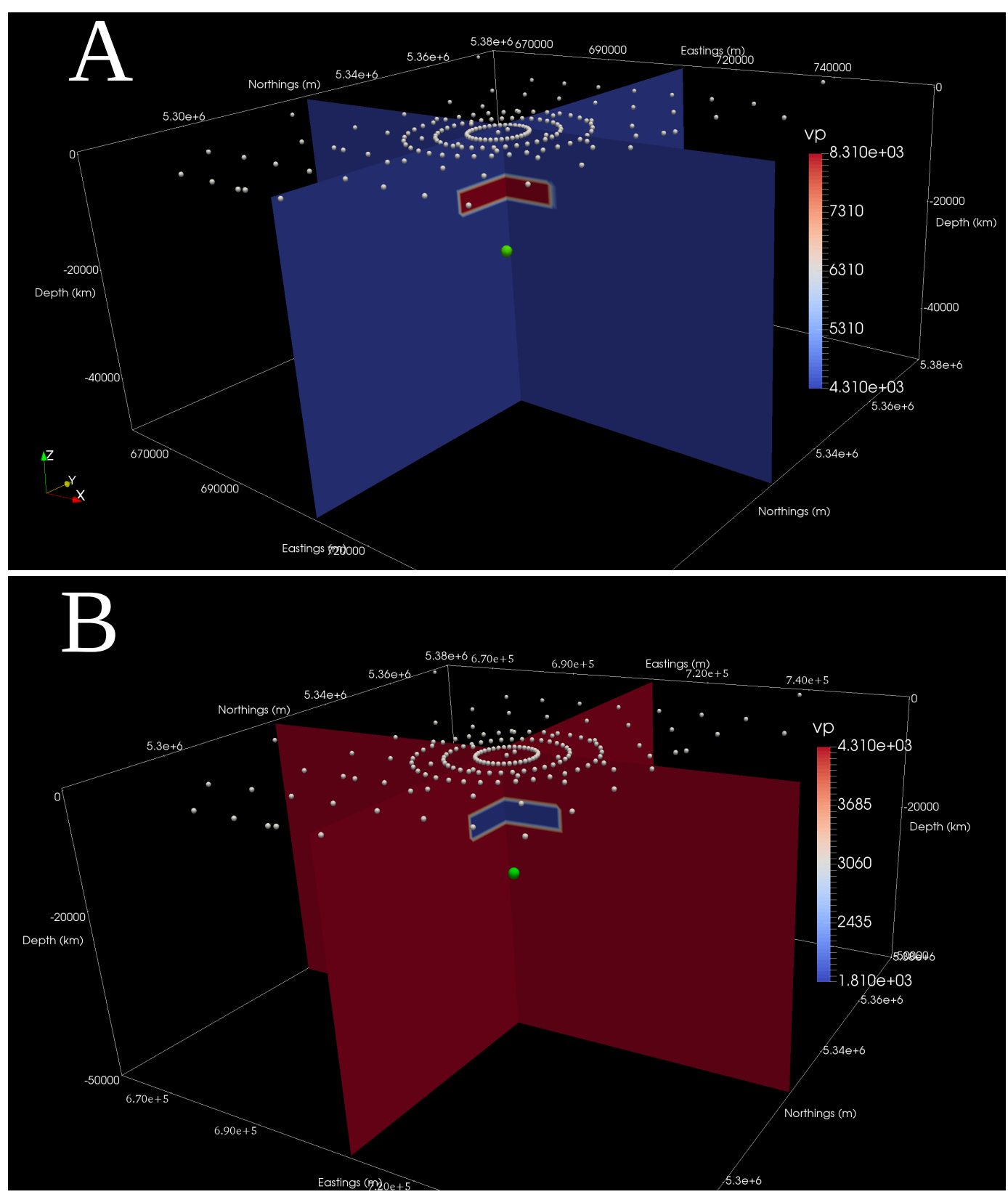

Figure 5.13: Illustration of the model used to test the effect of scatterers. (A) is high velocity anomaly (B) is the low velocity anomaly. The stations distribution are represented by the white spheres. The green sphere shows the source used. The colour scale represents the velocity. 


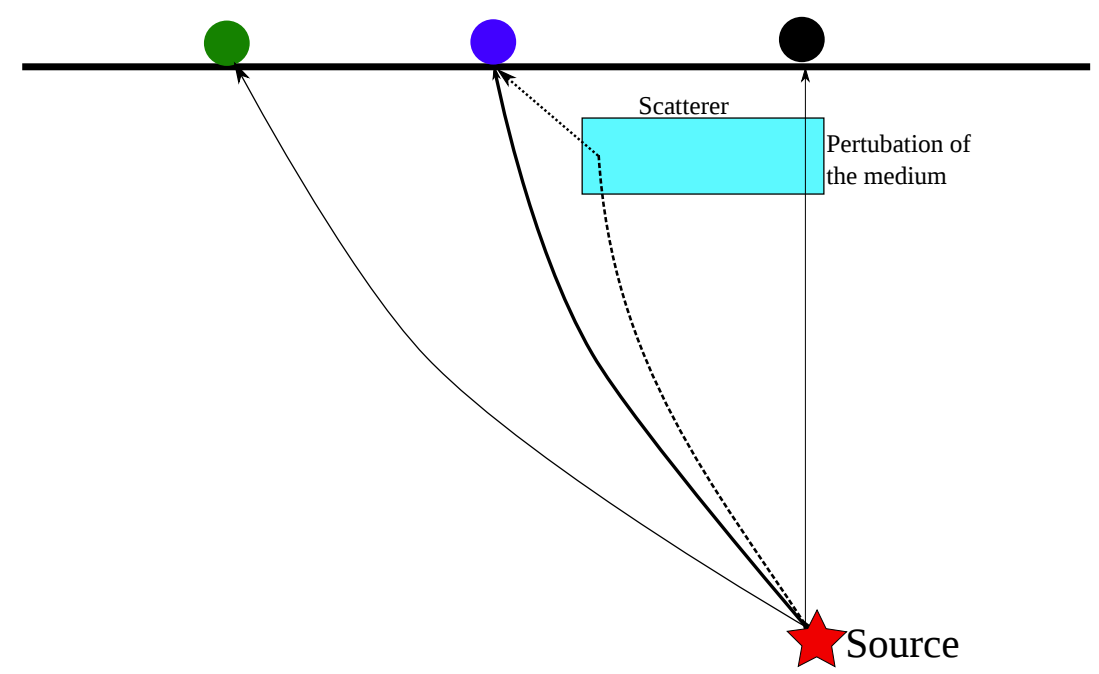

Figure 5.14: Schematic diagram of source station distribution used to test for effect of scatterers. The cyan coloured box represent the introduced scatterer. The black lines represents the ray-paths. The black, blue and green circles represents stations on-top, at the edge and far away from the scatterer respectively.

5.14). This waveform interaction clearly distorts the primary waveform and affects the arrival of the shear wave. The distortion in this case was a destructive interference where the amplitude of the waveforms decreased (defocused). We also observed waveform distortion when we used the low velocity scatterer (Figure 5.14B). Figure C.4, C.5 and C.6 of Appendix C shows waveforms for the low velocity anomaly. For the low velocity anomaly, the distortion we observed was likely constructive interference where the amplitude of the waveforms are focused (east component in Figure C.6).

To examine the effect of scatterers on SWS measurements, we estimated SWS measurements for the source receiver configuration shown in Figure 5.14. For each of the stations in Figure 5.14, we estimated $\delta t$ and $\phi$ for a range of back azimuths (from $0^{\circ}$ to $360^{\circ}$ ). Figures 5.18, 5.19 and 5.20 shows the estimated splitting parameters as a function of back-azimuth for stations (ring stations) directly above the scatterer, far away from the scatterer and at the edge of the scatterer respectively. For each plot we also show the two (low and high velocity) anomalous scatterers and the reference model (no scatterer) used in the simulation. For stations directly above the scatterer (see Figure 5.14 for the illustration of the station), we observed few variations in the SWS parameters (Figure 5.18). Both the low and high anomalies (magenta and yellow lines in Figure 5.18) show few variations from the reference model (cyan lines in Figure 5.18) 


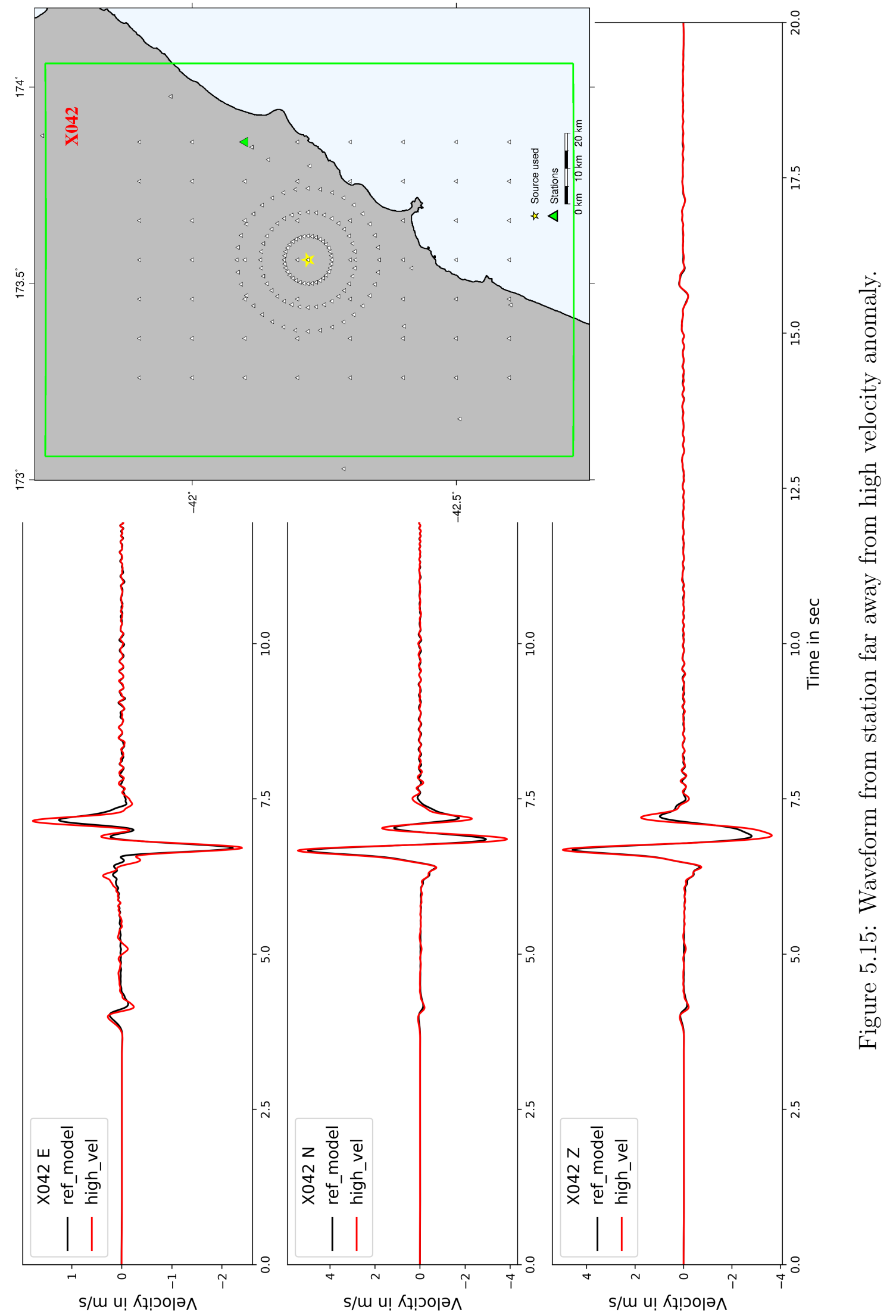




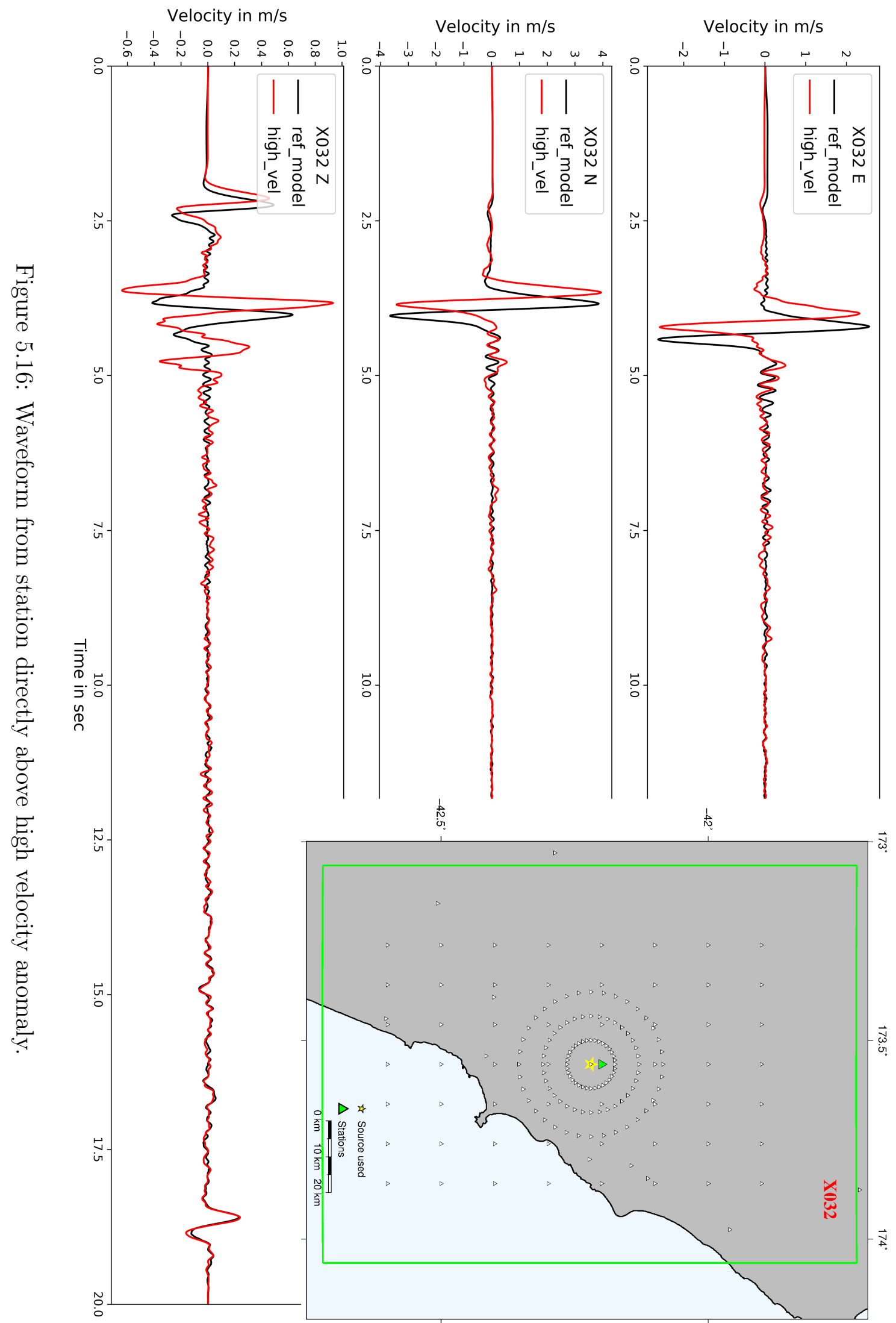




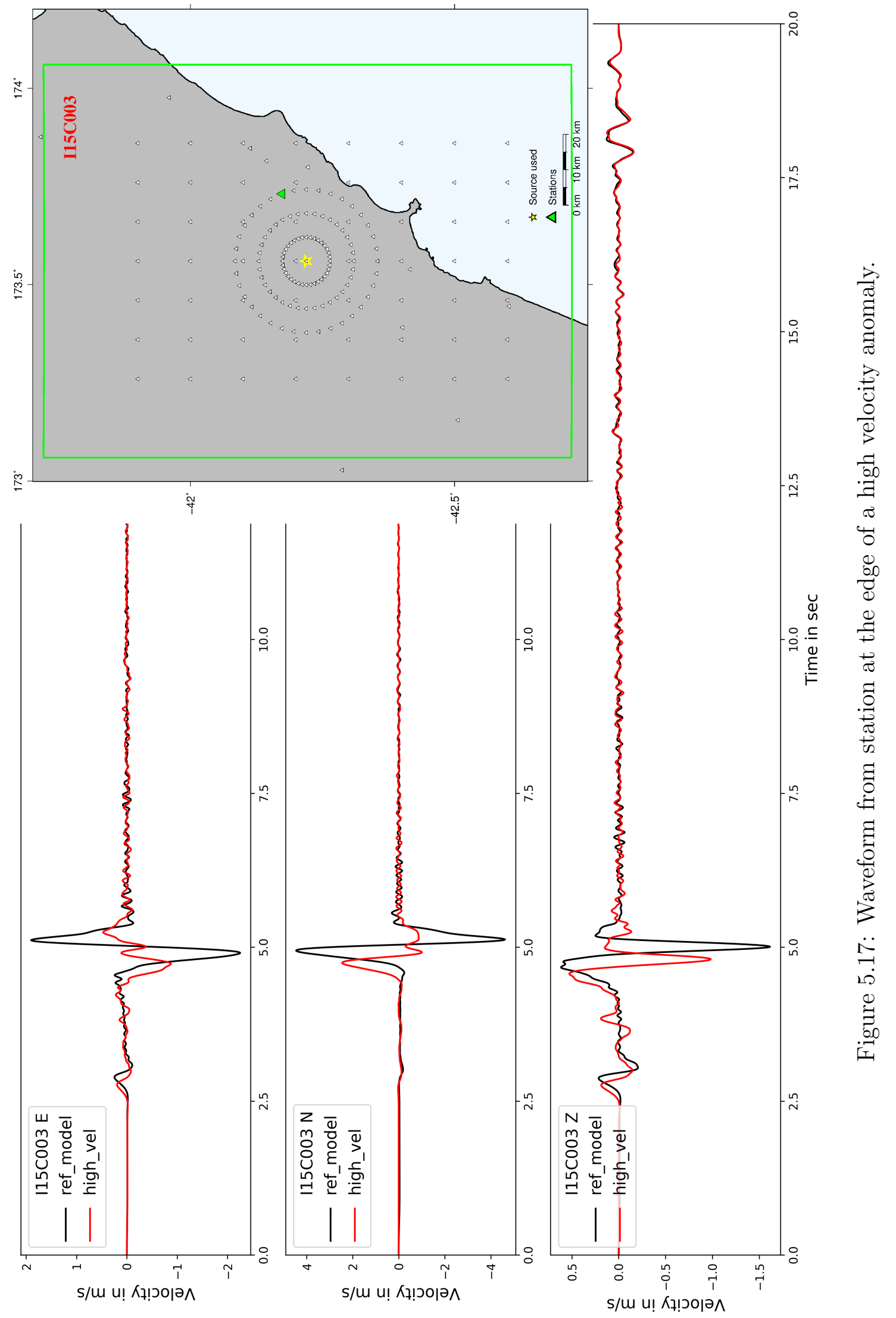


except for the peaks observed in Figure 5.18, which are null measurements. Similarly, less back azimuthal variation of $\delta t$ and $\phi$ was observed for stations far away from the scatterer (Figure 5.19). The smooth variations observed in Figure 5.19 are due to the high incidence angle $\left(\sim 26^{\circ}\right)$ of the incoming shear wave as discussed in Section 5.3.1. These variations are expected since the waveforms (see Figure 5.15) showed little variation.

However, we observed even more variation in the SWS parameters for stations at the edge of the scatterer (Figure 5.20). The observed variations are likely due to the interaction of the secondary waves with the primary waves as discussed earlier. The interactions clearly distorted the waveforms (Figure 5.17) and subsequently had a significant effect on the estimated splitting parameters. Examining the MFAST diagnostic plots for the reference model and the high velocity anomaly (Figure 5.21) at some of the stations revealed that the effect of the scatterer on the delay times could arise from cycle skipping. This occurs when the distorted waveform (destructive interference) causes a misfit between the two horizontal components (in the process of "unsplitting" the waveform). Figure 5.21B and $\mathrm{B}^{1}$ show that the expected delay time of $0.3 \mathrm{~s}$ (reference model) is increased to 1.0 (scatterer), an increase of $0.7 \mathrm{~s}$ which is the period of our simulated waveform. A waveform mismatch of the wave's period shows a clear phenomena of cycle skipping and it is confirmed in the particle motion of the fast and slow plot (see Figure $5.21 \mathrm{~A}^{1}$ ). We also analysed a similar experimental set up - using a smaller scatterer $1.5 \times 1.5 \times 1.5 \mathrm{~km}$ (compared to the $16 \times 16 \times 8 \mathrm{~km}$ rectangular anomaly used). The results showed less significant variations than with the larger scatterer. This was due the fact that our wavelength $(2.38 \mathrm{~km})$ was larger than the size of the scatterer and the waveform treats it like a homogeneous medium.

Clearly, heterogeneity in a medium can affect SWS measurements and this effect depends on the frequency of the shear wave and the size of the heterogeneity. This observation supports the suggestion of Aster et al. (1990) and Aster et al. (1991) that scatterers (due to inhomogeneity of a medium in the propagation path of the shear wave) combined with a temporal variation in the path may serve as the cause the variation in SWS parameters rather than the temporal variation in stress reported by 
Crampin (1990b). Crampin (1990b) reported on temporal variations in SWS parameter using local earthquakes recorded by the Anza network in southern California. Aster et al. (1990) and Aster et al. (1991) refuted their claims after analysing the same data and argued that physical mechanisms including scatterers cause spatial variation that is wrongly attributed to time variations. Castellazzi et al. (2015) also suggest that scatterers can have a strong effect on SWS parameters after analysing SWS parameters in Ruapehu, New Zealand. The significant variation in SWS parameter we observed due to a scatterer, supports the suggestions of Aster et al. (1990) and Castellazzi et al. (2015). Thus care needs to be taking when interpreting temporal variation of SWS parameters. 


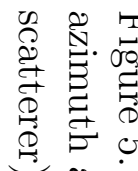

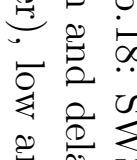

है

吉. 志. 范

है का

용

एँ त

क 롱.

足

节吉

की क

०。

政

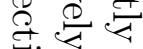

究总
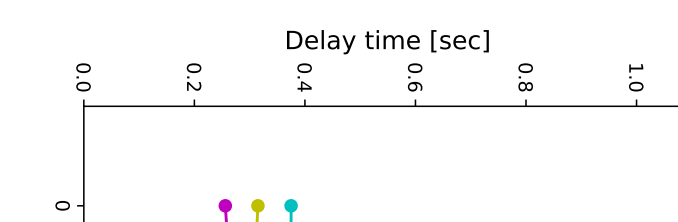

,

?.
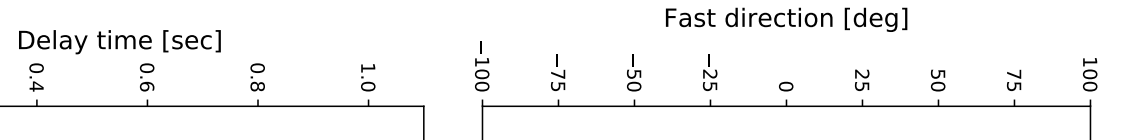

दे

है

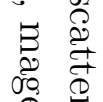

品

苛

पू

है

节 す

is

告官

क्ष

की

官

कृ

串

范

ง
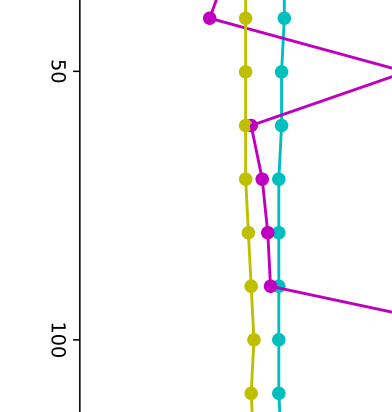

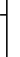

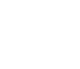

虫

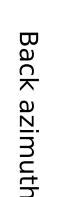

ธิ
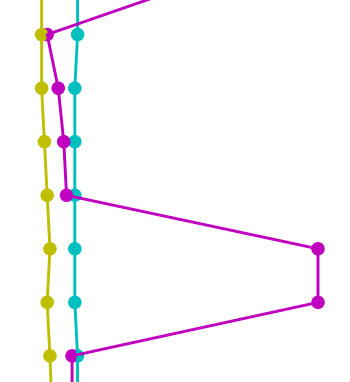

พั้

웅

क हैं

串

\& 2

훙.

के

है

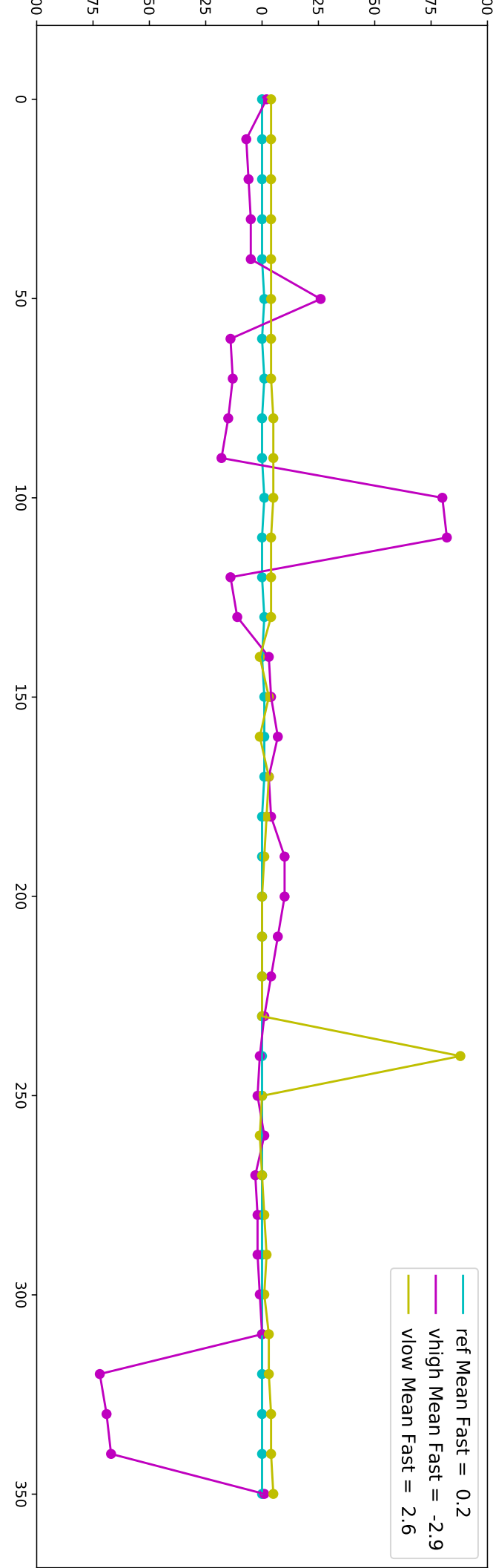




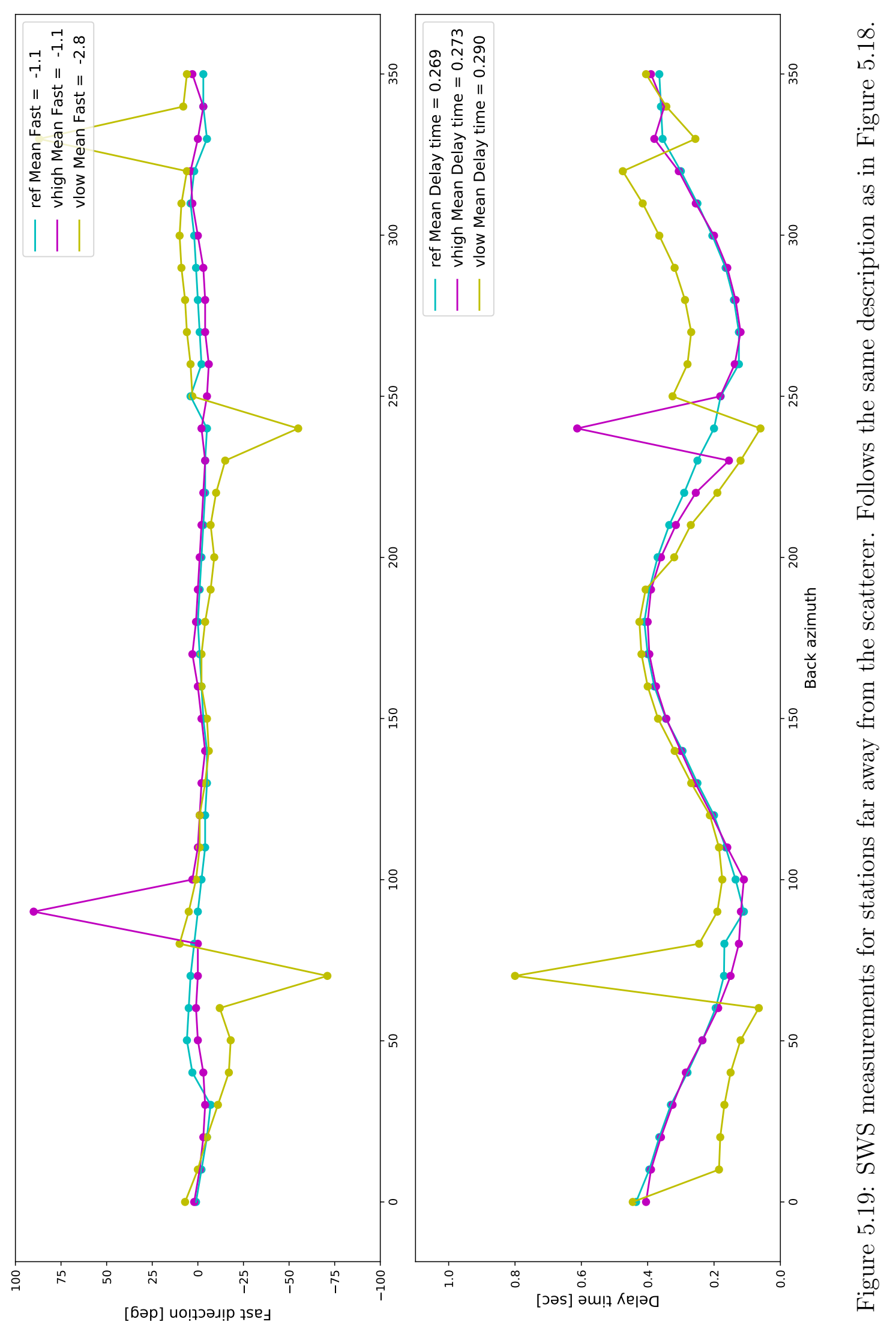




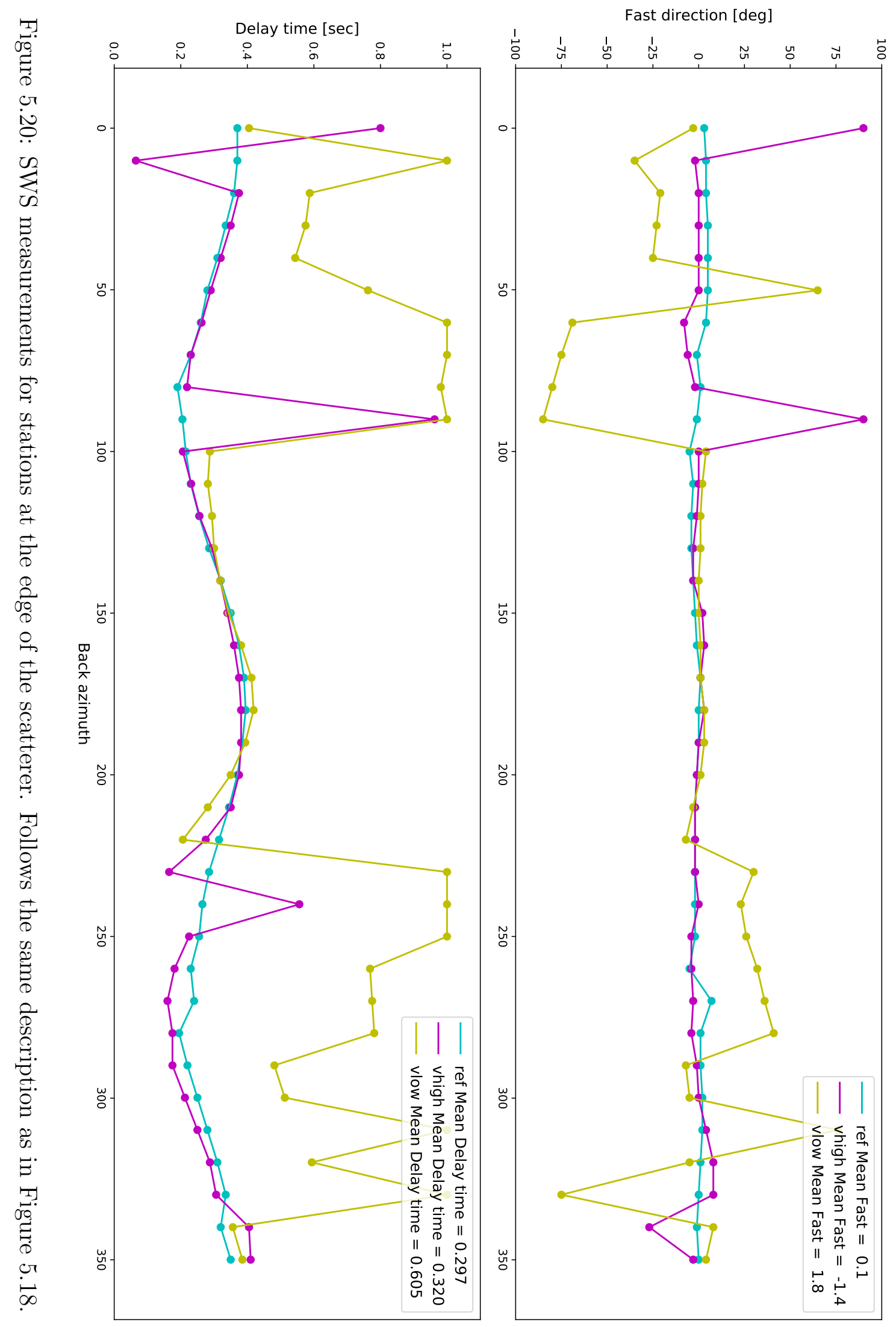



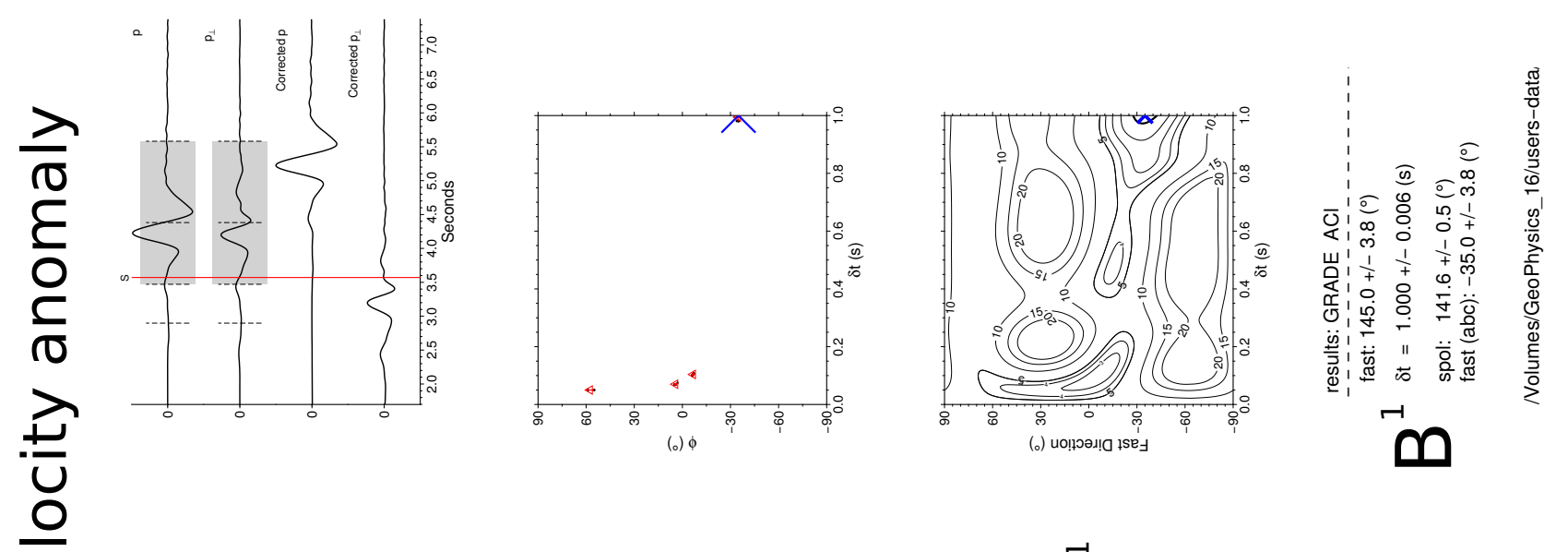

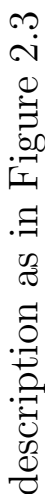
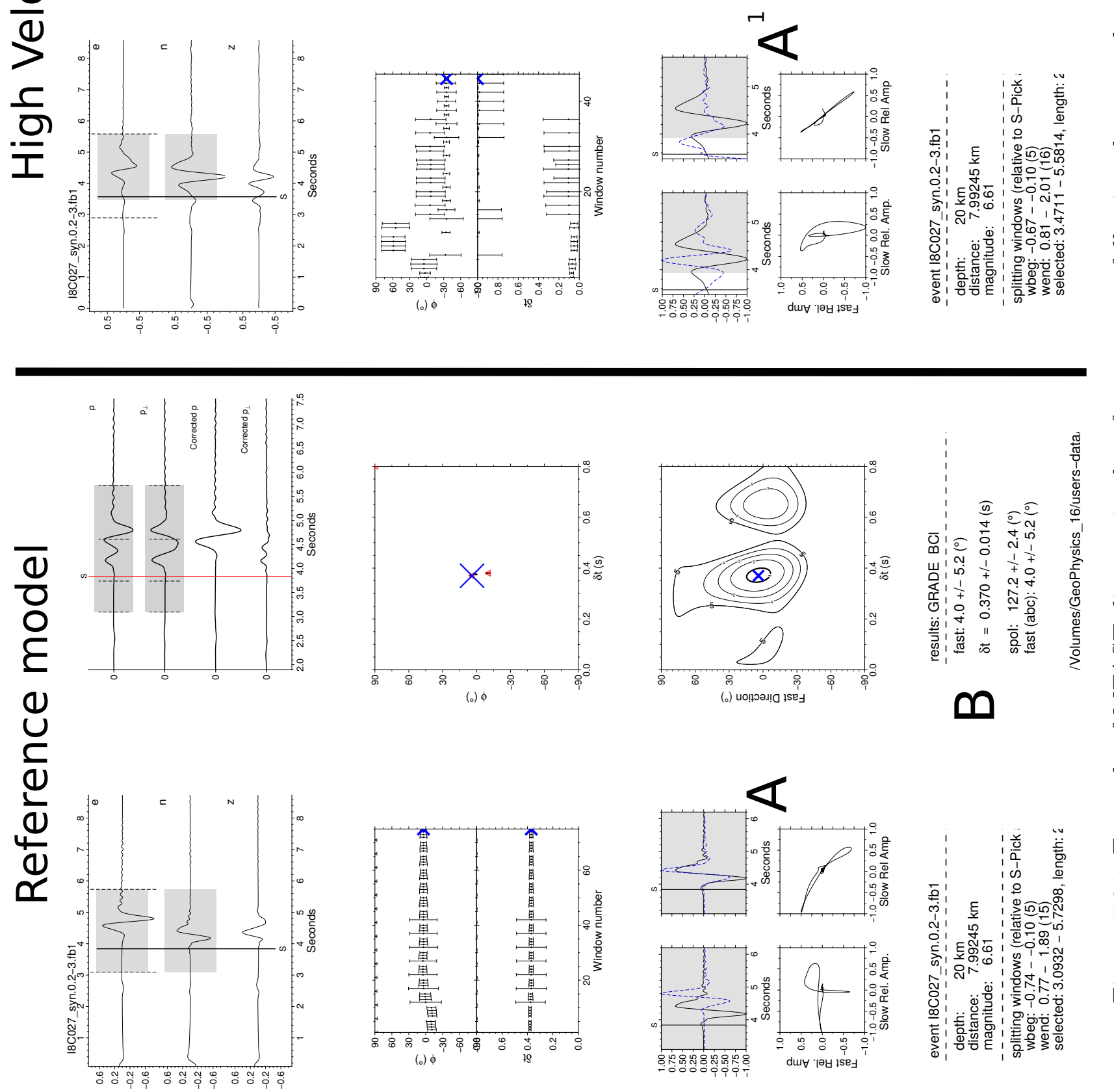

क्ष

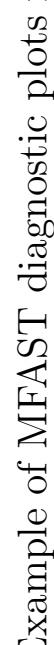
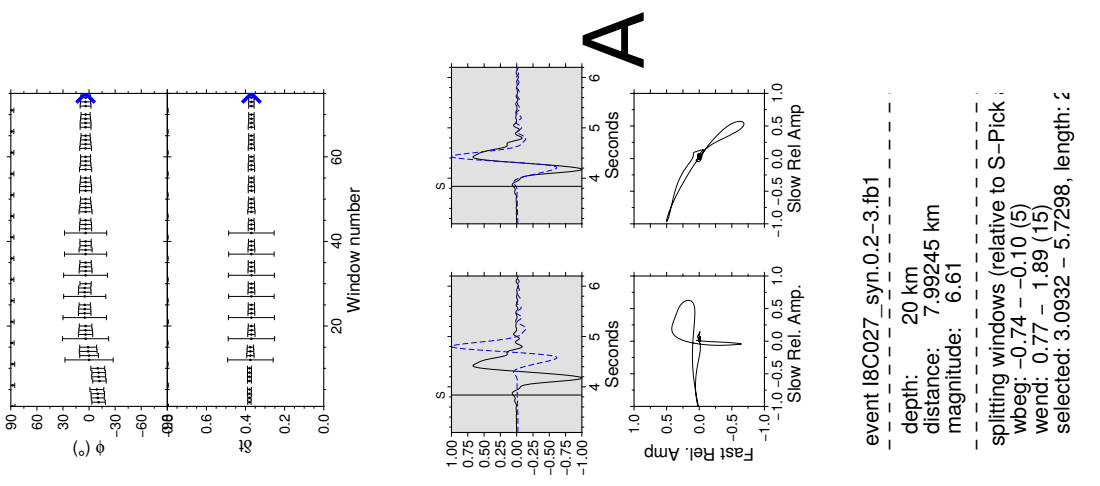

予

$\stackrel{9}{\$}$

.

흥

4

壱

\section{.}




\subsubsection{Effect of noise on SWS measurements}

Here we looked at the effect of noise on SWS measurements. We considered this by adding Gaussian noise to the simulated waveforms. We used waveforms simulated on a ring of stations close to the epicenter ( $2 \mathrm{~km}$ from the source at the surface). Figure 5.22 shows a plot of different noise level used to test the effect of noise. The waveforms are ordered according to the signal to noise ratio. We considered waveforms with signal to noise ratio, SNR, of 83.0, 32.1, 13.5, 8.8, 8.3 and 5.8. MFAST is set to discard waveforms with SNR less than 3. We decided to use a SNR of 5.8 as our minimum SNR value because analysis below this SNR gives similar splitting parameters or are discarded by MFAST.

From Figure 5.23 we do not observe a significant variation in the estimated splitting parameters with back azimuth as the signal to noise ratio of the waveforms decreases. This is likely due the fact that the high frequency Gaussian noise is filtered out by the set of predefined filters (see Table B.2 of Appendix B). Castellazzi et al. (2015) examined the effect of noise in SWS parameters in greater details using the anirec code (1D case). They considered Gaussian noise and two additional noise types; coda noise and background noise. They came to a similar conclusion that the noise level has less or no significant effect on SWS measurements since they are often filtered out or discarded from analysis. We recommend that a future attempt should be made, in a 3D case (using SPECFEM3D), to consider the effect that coda and background noise on SWS measurements as considered by Castellazzi et al. (2015) in a 1D case. 


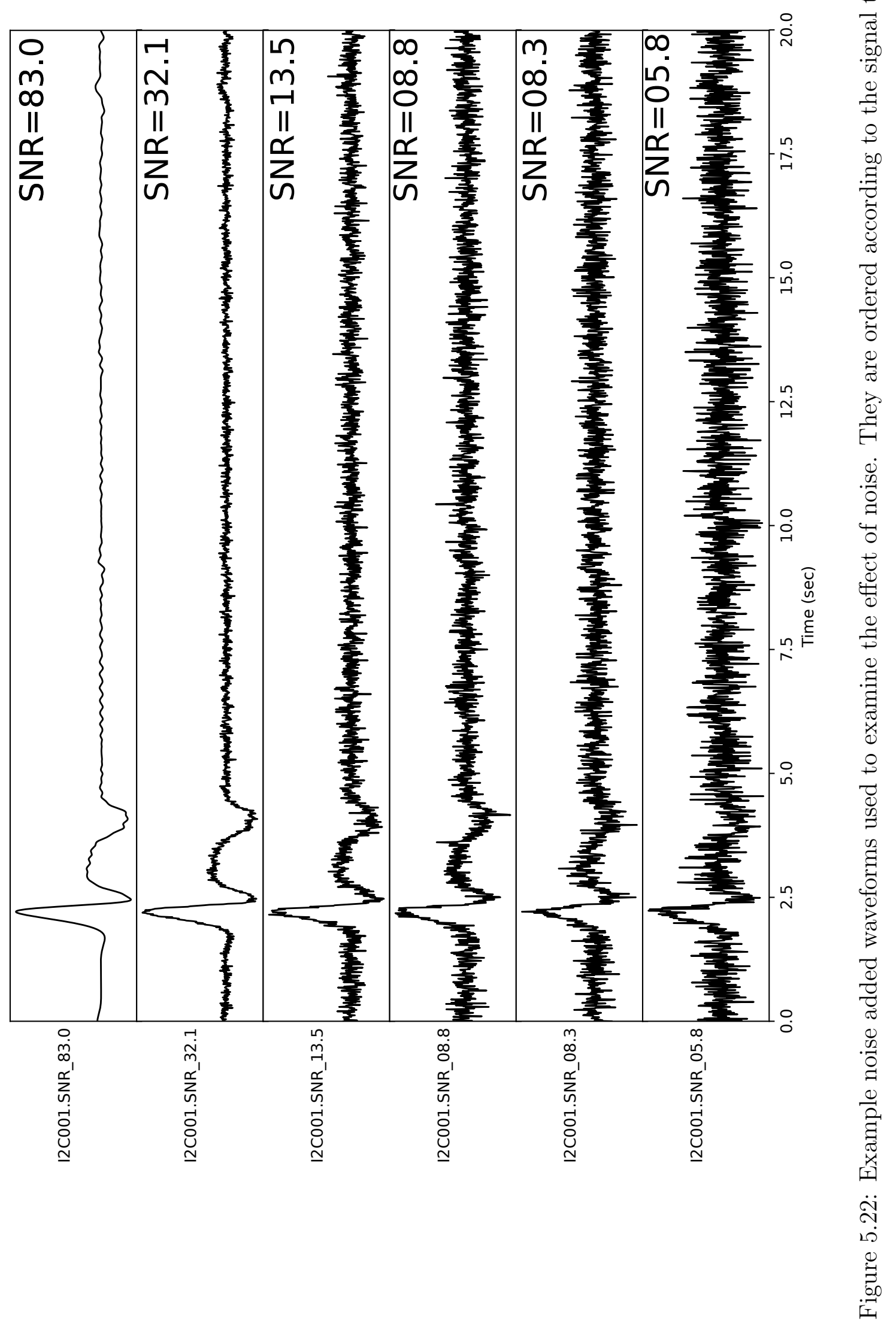




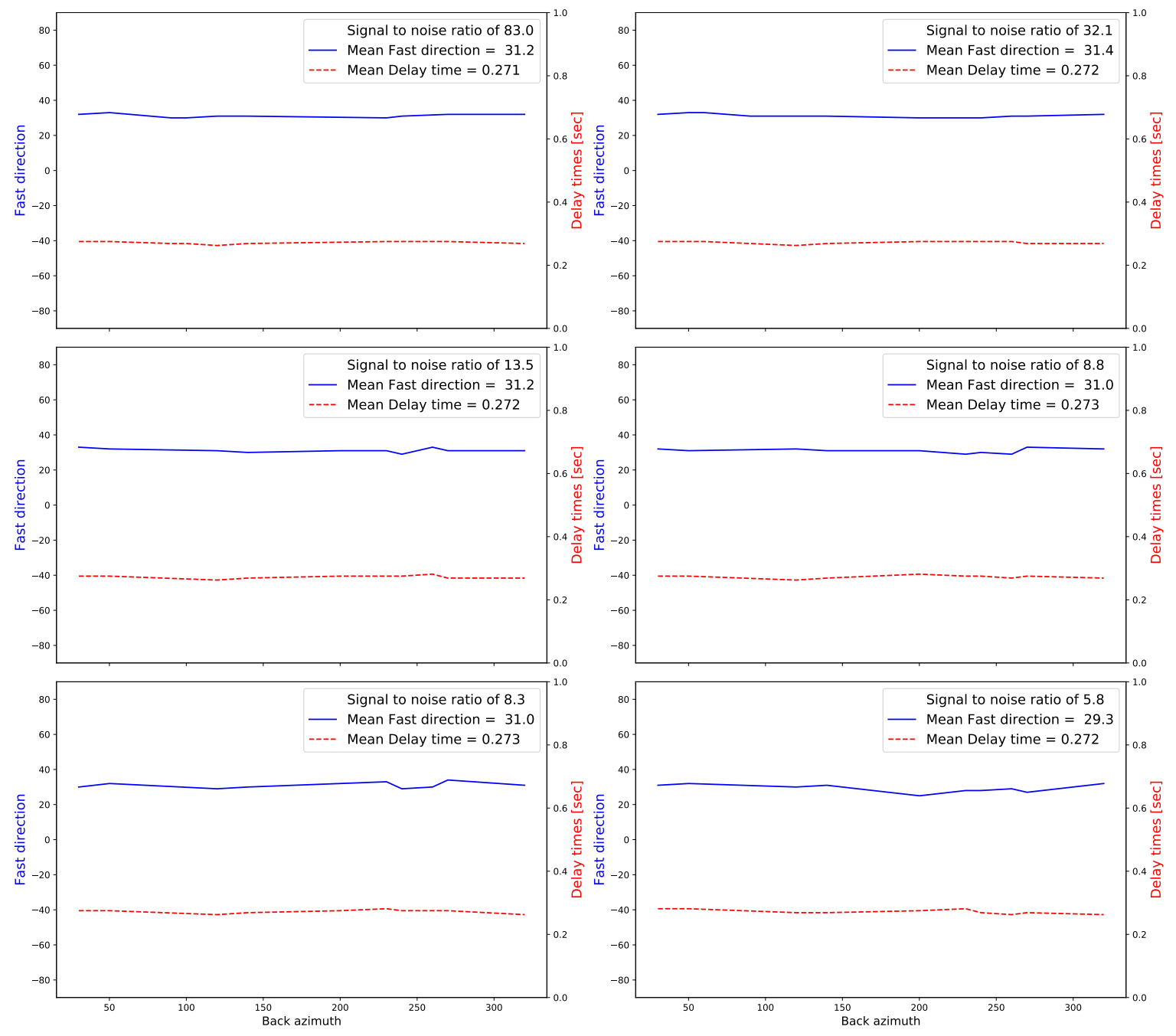

Figure 5.23: A plot of comparison of apparent splitting parameters as a function of back-azimuth ranging from $0^{\circ}$ to $360^{\circ}$ for the $3 \mathrm{D}$ case for different noise level. There is no significant variations as the signal to noise ratio of the waveforms decreases. 


\subsection{Conclusions}

We presented ongoing research to investigate how certain proposed physical mechanism affect shear wave splitting measurements. We used the SpecFEM3D programe to simulate waveforms (with frequency up to $1.33 \mathrm{~Hz}$ ) propagating through an anisotropic medium for a variety of scenarios to investigate the effect that (1) incidence angle of the incoming shear wave, (2) variation in earthquake source mechanisms, and (3) scatterers have on SWS parameters. For the effect of incidence angle of the incoming shear wave, we observed that for larger angles of incidence (more than $35^{\circ}$ ), the estimated SWS parameters are unstable and unreliable. This effect was also confirmed when we used synthetic waveforms in a 1-D medium. The variations are due to the body-wave phase conversion at the surface (Nuttli, 1961; Liu and Crampin, 1990; Crampin, 1985; Crampin and Booth, 1989; Crampin and Gao, 2006).

In testing the effect of source mechanisms on SWS we used several focal mechanisms from a simple normal fault to an oblique source mechanism like the $2016 \mathrm{M}_{w} 7.8$ Kaikōura earthquake. The variation of source mechanism showed less significant effect on SWS measurements, except for null measurements (when the incoming polarization direction is parallel to the fast or slow direction). Analyses of the effect of scatterers (anomalous elastic property) on $\delta t$ and $\phi$ revealed a significant variation in SWS parameters. We observed that SWS measurements from ray paths passing close to the edge of a scatterer showed unstable results and distorted waveforms compared to stations directly above the anomaly and far away from the anomaly. We also observed that the effect of a scatterer depends on the wavelength of the incoming waves, because a shear wave with a longer wavelength (compared to the size of the anomaly), considers the medium as effectively homogeneous. Based on our observations, we conclude that for crustal SWS measurements, only non-null SWS parameters with angle of incidence less that $35^{\circ}$ should be considered for interpretation, as measurements outside these are unstable and unreliable. Also, we suggest that care needs to taken when interpreting temporal variations of SWS measurement since spatial variations such scatterers can introduce variations which can be misconstrued as temporal variations. For temporal 
variation interpretations, we recommend using events with similar propagating path (repeating or near repeating events) that are sampling the same medium. 


\section{Summary}

The overarching goal of this thesis is to address the question: "What physical processes and/or mechanisms control or affect measurement of crustal seismic anisotropy through shear wave splitting?". We used two approaches, empirical observations and numerical simulations, to answer this research question. For each of the approaches we posed a series of questions which are answered in each individual Chapter. This Chapter summarises our main findings. We start with the two empirical studies and conclude with the numerical simulation study.

For our first empirical study, Chapter 3, we performed a detailed spatial and temporal analysis of shear wave splitting measurements to investigate what controls the anisotropic structure in a complex tectonic setting where there is subduction merging to strike-slip deformation amid the complex Marlborough Fault System, central New Zealand. This study aimed to answer the following questions: (A) What is the lateral and vertical extent of the anisotropic structures in central New Zealand? (B) What physical processes control seismic anisotropy in a complex tectonic region? Does gravitational stress, as a result of strong topography contrast, control seismic anisotropy? (C) Did the 2013 Cook Strait earthquake and the $2016 \mathrm{M}_{w} 7.8$ Kaikōura earthquake sequences affect the anisotropic structure or the state of anisotropy in central New Zealand?

In answering these questions, we calculated SWS parameters for over 40,000 crustal earthquakes recorded on 36 seismic stations spanning close to $5 \frac{1}{2}$ years between 2013 and 2018. We made use of the increase in seismicity due to the 2013 Cook Strait (Seddon, Grassmere) and 2016 Kaikōura earthquakes sequences in New Zealand. Due to the large size of the dataset, we used an automatic, objective splitting analysis algorithm and automatic local S-phase pickers to expedite the processing and to minimize 
observer bias. We obtained a total of 102,260 (out of a possible 398,169) high-quality measurements. To investigate the lateral extent of seismic anisotropy in central New Zealand, we used the spatial averaging technique by Johnson et al. (2011) to spatially average the 102260 fast direction measurements. Significant lateral variation of fast direction was observed. Two of these spatial variations are (1) the dominant NE-SW trend, which is sub-parallel to the strike of the major active faults and also to the topographic trends and (2) a contrasting NW-SE feature around the eastern end of the Marlborough Fault System near Kaikōura. This latter feature could not be explained either by the fault orientations or the tectonic stresses.

To examine the vertical extent of the anisotropic structures in central New Zealand, we analyzed the association between delay time, hypocentral depth and epicentral distance at all 36 individual stations. The correlation coefficients at the analyzed stations were less than 0.2 , indicating a lack of clear correlation between delay time and hypocentral depth or epicentral distance. This is an indication that the observed anisotropic structure is confined to the upper few kilometers of the crust. In summary, the observed significant lateral variations is an indication that the anisotropic structure is likely controlled by more than one mechanism.

This brings us to the next question about what physical processes/mechanisms control seismic anisotropy in a complex tectonic region as central New Zealand. To answer this question, we made quantitative comparisons of our spatially averaged $\phi$ measurements with GNSS derived principal contraction axes, average fault orientations, gravitational stress and focal mechanism inversion measurements. To distinguish between stressinduced and structurally controlled seismic anisotropy we quantitatively compared our spatially averaged $\phi$ measurements to the average orientation of the closest active fault segments. This analysis revealed regions which are structurally controlled and regions controlled by stresses. We also examined how both tectonic stresses (from focal mechanism inversion) and gravitational stresses (due to the topographic difference betweeen the Kaikōura ranges and the Kaikōura canyon) best explain the regions identified to be controlled by stresses. Similar quantitative comparisons were done for tectonic stresses and gravitational stresses. The high correspondence between $S_{H \max }$ calculated 
from gravitational potential energy from topography and average fast polarization orientation around the Marlborough and Kaikōura region suggests that gravitationally induced stresses control the anisotropic structure in the region. The general disagreement observed for the tectonic stresses and spatially averaged $\phi$ measurements and the fact the observed anisotropy is confined to the upper few kilometers suggest that the tectonic stresses are acting on deeper structures than the measured anisotropic structure.

The last question posed was to investigate if the 2013 Cook Strait earthquake and the $2016 \mathrm{M}_{m} 7.8$ Kaikōura earthquake sequences affect the anisotropic structure or the state of anisotropy in central New Zealand. We systematically searched for temporal variations in delay time and calculated $V_{p} / V_{s}$ associated with the 2013 Cook Strait and 2016 Kaikōura earthquakes. Temporal analysis of stacked measurements from all 36 stations revealed apparent temporal variations, but measurements from a cluster of closely spaced earthquakes showed no temporal variations. Also, analysis of measurements with similar back azimuths revealed no clear temporal variation. This indicates that the apparent temporal variations observed are likely caused by variation in earthquake path through spatially varying media. We also quantitatively compared averaged $\phi$ measurements between events before and after the 2016 Kaikōura earthquake to observe any spatio-temporal variation associated with the 2016 Kaikōura mainshock. The general agreement between measurements before and after the 2016 Kaikōura earthquake, is an indication that the state of anisotropy in central New Zealand has not changed due to the Kaikôura earthquake. The absence of temporal variations in $\phi$ suggests that any change in the stress field around central New Zealand produced anisotropy changes that are small compared to structural anisotropy. In summary, there are no significant temporal variations in delay time, $V_{p} / V_{s}$ and the state of anisotropy over the $5 \frac{1}{2}$ year period despite the occurrence of significant seismicity.

For our second empirical study, Chapter 4, we used SWS measurements to investigate the anisotropic structure of a fault considered to be approaching failure (the Alpine Fault of New Zealand). To unravel the processes that control the anisotropic structure within the Whataroa Valley, we used local earthquakes close to the Whataroa Val- 
ley that were recorded on 159 three component (3C) seismometers (with inter-station spacing $\sim 30 \mathrm{~m}$ ). In this study we aimed to answer the following questions: (1) What controls the anisotropic structure around the Whataroa Valley in the vicinity of the Alpine Fault? (2) What is the anisotropic structure as you move across the fault from the hanging wall to the foot-wall? and (3) What is the spatial variability of seismic anisotropy in the Whataroa Valley?

To answer the question of whether significant spatial variation in the observed anisotropy exists as we cross the Alpine Fault from the footwall to the hanging wall, we carried two averaging analyses along a perpendicular line across the Alpine Fault. Generally, we did not observe any significant departure of either delay time or fast direction from the mean values as we move perpendicularly across the Alpine Fault, suggesting an insignificant spatial variability in seismic anisotropy within the Whataroa Valley. The consistent NE-SW direction of the averaged $\phi$ for every $200 \mathrm{~m}$ step perpendicularly across the Fault is also an indication that there is no significant spatial variation of the observed anisotropy in the Whataroa Valley.

To investigate the physical processes controlling the anisotropic structure in the Valley, we compared the mean orientation of $\phi$ with structural data sets such as the orientations of fractures and foliations obtained from the acoustic borehole televiewer installed in the DFDP-2, the orientation of foliations within the Whataroa, and the orientation of the Alpine Fault trace. We observed an overall mean orientation of the fast direction that is near parallel to the orientation of the structural data sets, indicating that the anisotropic structure in the Whataroa Valley is mainly structural, and therefore the hypothesis of structural control of anisotropy best explains our results. We also observed that the orientation of horizontal tectonic stresses is at a high angle to the mean fast directions, which indicates that the anisotropic mechanism in the Valley is likely not related to the stress at the depth of the earthquakes. In summary, there was no significant spatial variation of the shear wave splitting parameters as we move across the Alpine Fault trace from the hanging wall to the footwall and the anisotropic structure within the Valley is structurally controlled by the Alpine Fault fabric, foliations and fractures within the Valley. 
For our last investigation, Chapter 5, we simulated the propagation of seismic waves in a variety of scenarios (using both 1-D and 3-D techniques) to investigate the possible physical mechanisms that likely cause variations in SWS measurements. Our synthetic scenarios were designed to answer the following research questions: (A) How does varying the angle of incidence of the shear wave affect SWS measurements? (B) How do varying fault source orientation affect SWS measurements? (C) How do scatterers affects SWS measurements?

In the quest of resolving the ultimate incidence angle below which SWS measurements are reasonably stable and reliable, we examined how varying angle of incidence of the shear wave affect SWS measurements using both a 1D and 3D methods. Our analysis using both methods, revealed that for larger angles of incidence $\left(>35^{\circ}\right)$, the estimated SWS parameters are unstable and unreliable compared to measurements estimated with angles of incidence $\left(<35^{\circ}\right)$. We simulated waveforms with different focal mechanisms to test the effect of source mechanisms on SWS measurements. The estimated SWS parameters from these waveforms showed less variations, except when the incoming polarization direction (which is determined by the source mechanism and the station location) is parallel to either the fast or slow direction (measurement considered as null). In examining how scatterers (anomalous elastic property) affect SWS measurement, we compared observations at stations placed directly above, at the edge and far away from an anomalous body. We observed that $\delta t$ and $\phi$ estimated from ray paths passing close to the edge of a scatterer showed unstable results and distorted waveforms compared to stations directly above the anomaly and far away from the anomaly. We also observed that the effect of a scatterer depends on the wavelength of the incoming wave, because when we used wave with a longer wavelength (compared to the size of the anomaly), the wave considers the medium as effectively homogeneous. In summary the incidence angle of the incoming shear wave, and scatterers, have significant effect on estimated SWS parameters. We suggest that for crustal SWS measurements, only non-null SWS parameters with angle of incidence less that $35^{\circ}$ should be considered for interpretation, as measurements outside these are unstable and unreliable. Also, we suggest that care needs to be taken when interpreting temporal 
variations of SWS measurement since spatial variations such as scatterers can introduce variations which can be misconstrued as temporal variations. For temporal variation interpretations, we recommend using events with a similar source-receiver propagating path (repeating or near repeating events). These sample a similar medium on the propagating path from source to receiver and will have more stable, repeatable results, and are therefore sensitive to genuine temporal variations in the medium.

In answering the question "What physical processes and/or mechanisms control or affect measurement of crustal seismic anisotropy through shear save splitting?", this study has shown that seismic anisotropy in the crust can be controlled by several physical processes (such as faults, fractures, foliations and tectonic stresses which are often reported in literature), but perhaps most importantly, we have shown that gravitational stresses due to topographic contrast can control seismic anisotropy. We recommend that examining the effect of gravitational stresses on crustal seismic anisotropy should not be neglected in future studies. We also show through simulation studies that scatterers and the incidence angle of the incoming shear wave have significant effects on the estimated SWS parameters.

\subsection{Future research}

Chapter 5 contains a summary of ongoing research into how physical mechanisms can affect shear wave splitting measurements. Chapter 5 provides a framework for further study of the mechanisms that affect SWS measurement. We showed that two of the three mechanisms considered significantly affect SWS measurements. Aside from these three mechanisms, there are others that should be examined, such as effect of topography, dipping layers, different symmetry axes and multiple layers of anisotropy. Existing literature suggest that the observations of shear waves recorded at the free surface are often contaminated by second wave arrivals following the initial P-wave arrival (P-coda) (Booth and Crampin, 1985; Crampin, 1981; Liu and Crampin, 1990; Rodgers et al., 2010). The Earth's surface topography acts as a reflecting surface for an upward propagating seismic wave-field. The rugged surface can scatter, focus or defocus 

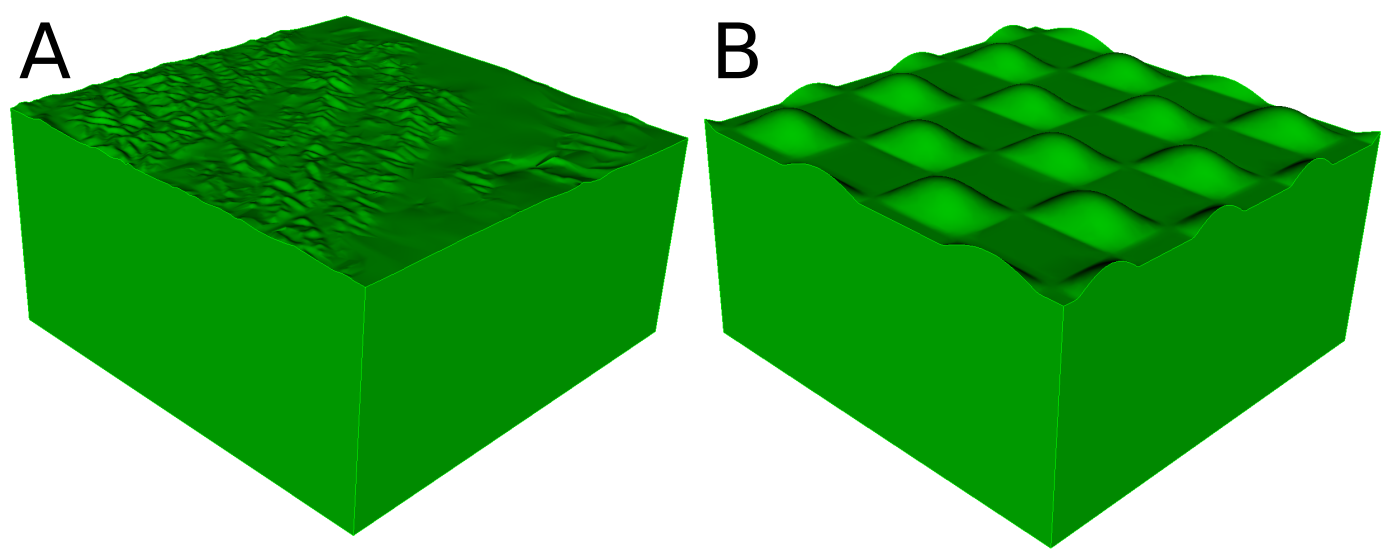

1

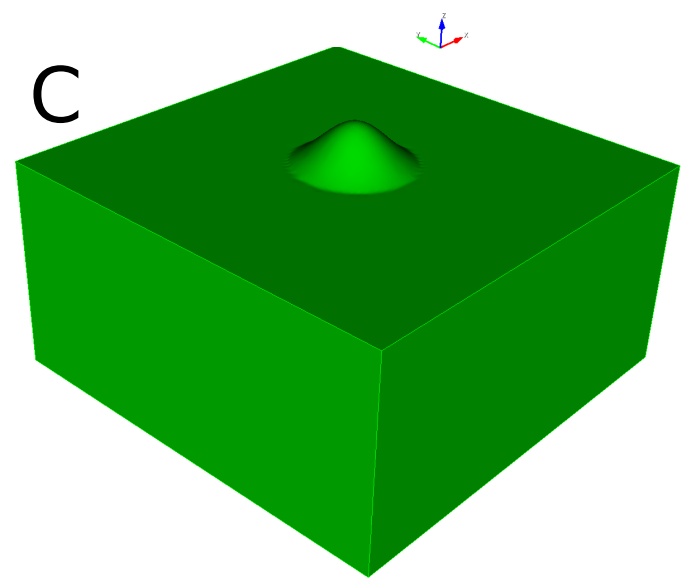

1.

Figure 6.1: Examples of suggested topographic models used. (A) is a real topographic model, (B) is a sinusoidal model and $(\mathrm{C})$ is hill at the center of the model.

the propagating waves, and thereby amplify or de-amplify the seismic response (Boore, 1972; Booth and Crampin, 1985; Lee et al., 2009a,b). This spatial variation in seismic response can convert body-waves to surface waves and vice versa (Boore, 1972; Lee et al., 2009a,b; Rodgers et al., 2010). This phase perturbation can lead to the generation of unwanted contaminating arrivals both before and after the main shear-wave phase. These additional arrivals may critically modify the characteristics of the observed shearwave, and can subsequently affect SWS measurements. Thus the need to test for the effect of topography on SWS measurements. In testing this effect one could use a realistic topography model or an artificial topography (for example sinusoidal peaks distributed across a model or a hill placed at the center of a model). Figure 6.1 shows possible examples of topographic models that could be used. Testing for the effect of scatterers can also be extended to consider randomly distributed scatterers. 
Based on our findings that gravitational stresses controls seismic anisotropy, we recommend testing this finding through simulation studies, both in terms of it scattering capability as well as it change in stress.

We also recommend attempt should be made to use repeating or near repeating event to search for temporal variation in SWS parameters associated with the 2013 Cook Strait (Seddon, Grassmere) and 2016 Kaikōura earthquakes in New Zealand. We recommend attempt should also be made to utilize the detailed earthquake catalog contracted by Michailos et al. (2018) to characterize the anisotropic structure and infer the likely mechanism that controls the anisotropy around the entire central section of the Alpine fault. 
Appendices 



\section{A Supporting Information for Chapter 3}

"Spatial and temporal Analysis of Seismic Anisotropy Associated with the Cook Strait and Kaikoura Earthquake Sequence in New Zealand"

\section{Introduction}

This section contains supplemental materials that augment reader's understanding of Chapter 3. It contains one table and 11 figures. 


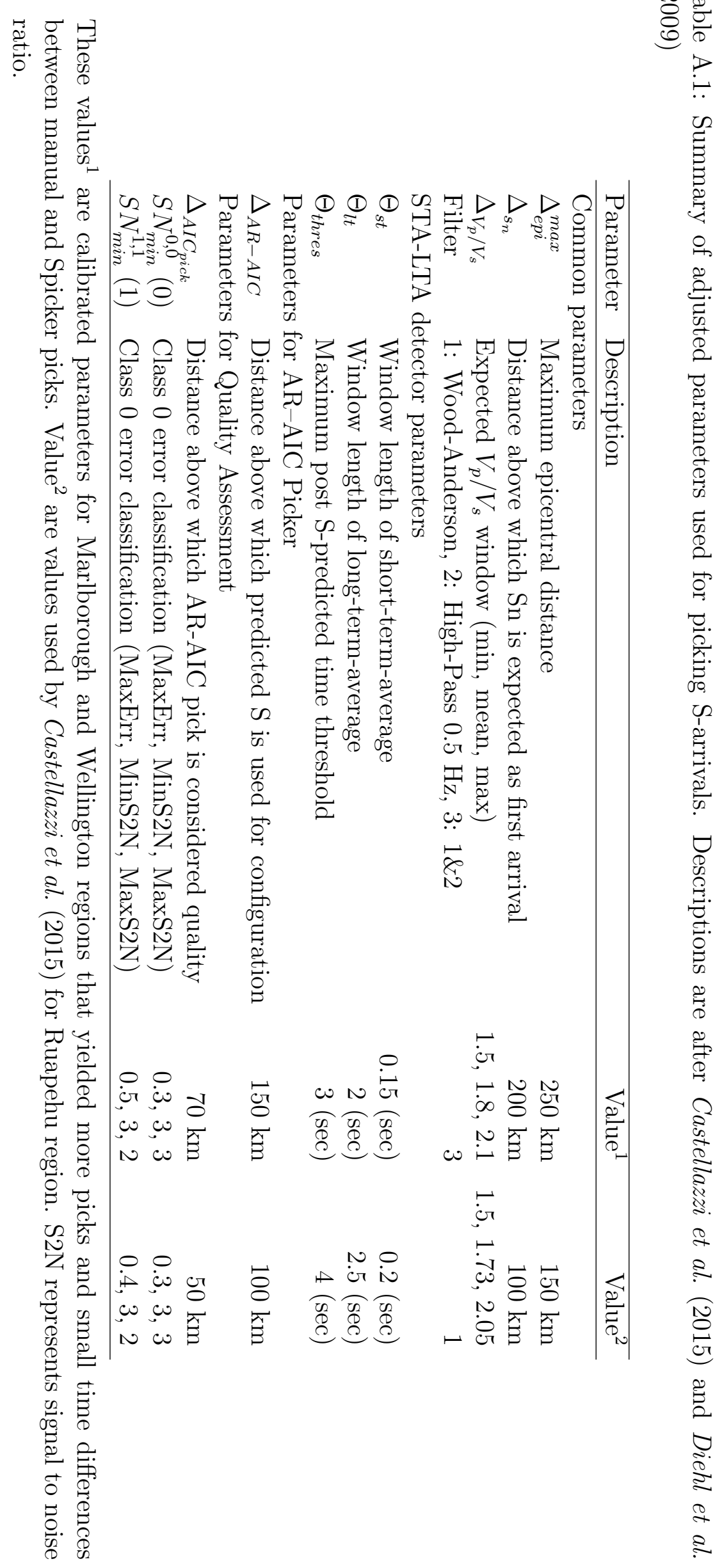



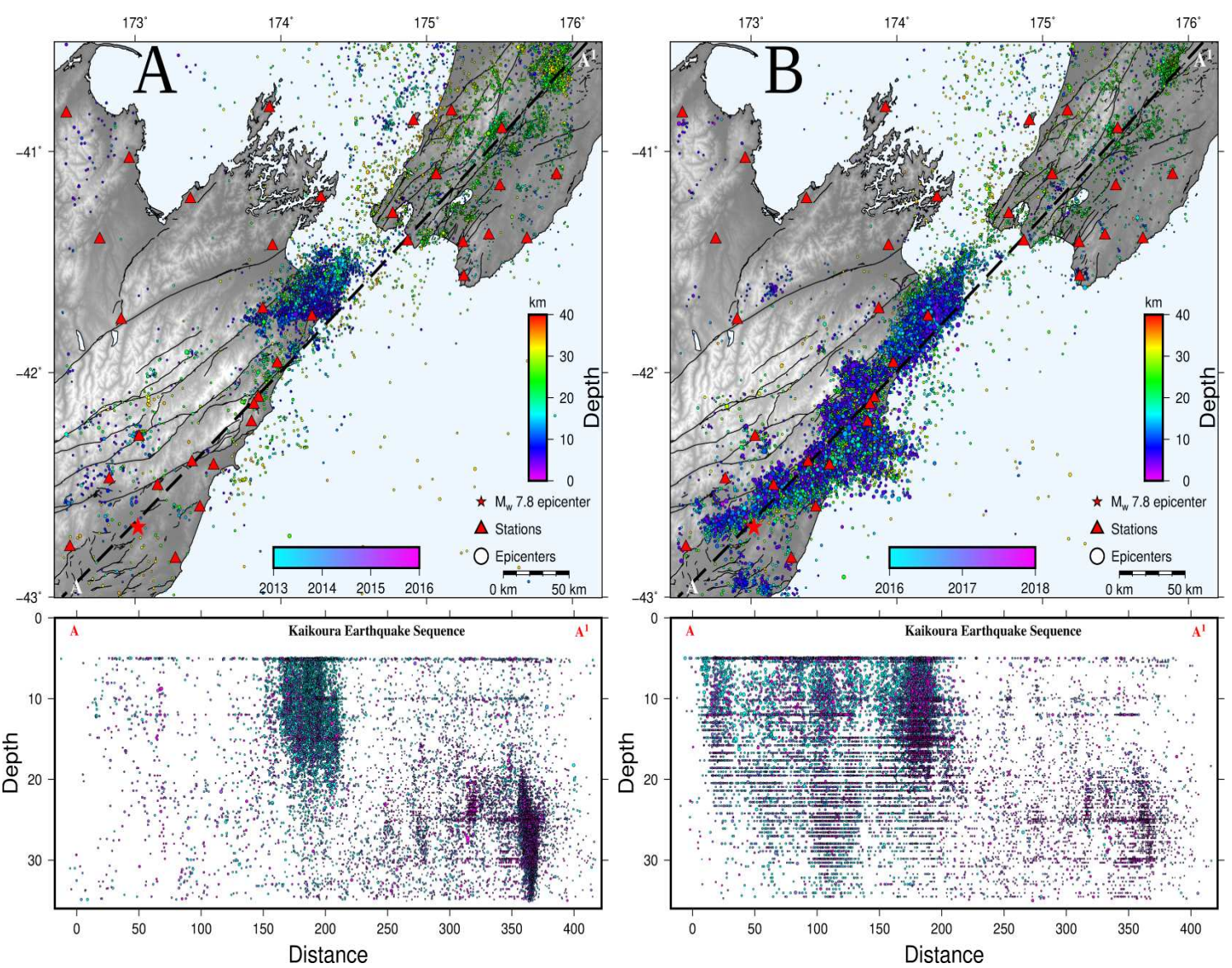

Figure A.1: Map of events (A) before and (B) after the 2016 Kaikōura earthquake: Map view with epicenters of earthquakes before (left) and after (right) the $M_{w} 7.8$ Kaikoura Earthquake. Red star shows the $M_{w} 7.8$ epicenter with the red triangles showing the GeoNet stations in the region. Events are scaled according to their magnitude, and colour coded according to their depth. A cross-section from A to $\mathrm{A}^{1}$ shows the depth distribution and colored by time (time scale on main Figure). 


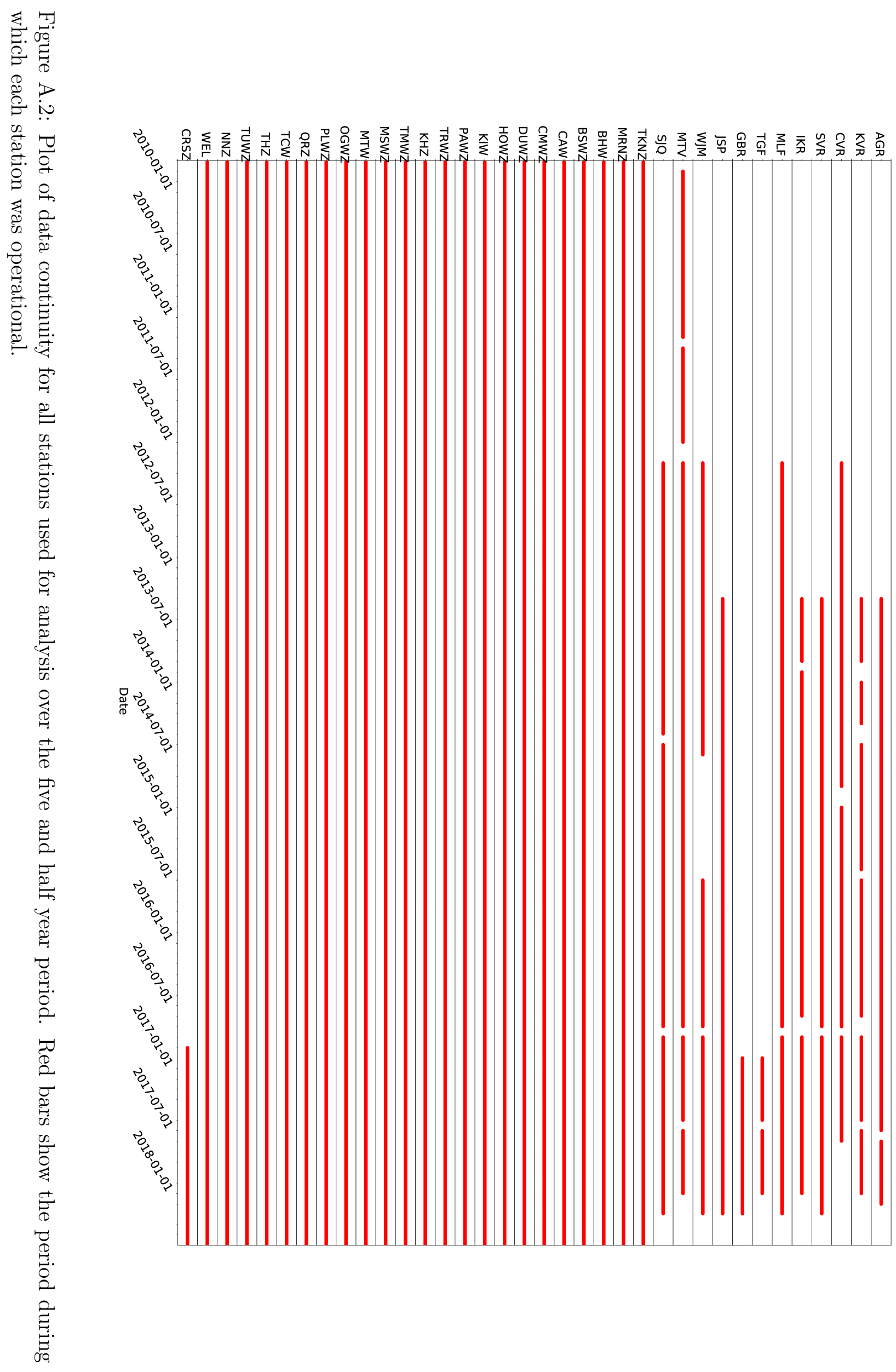



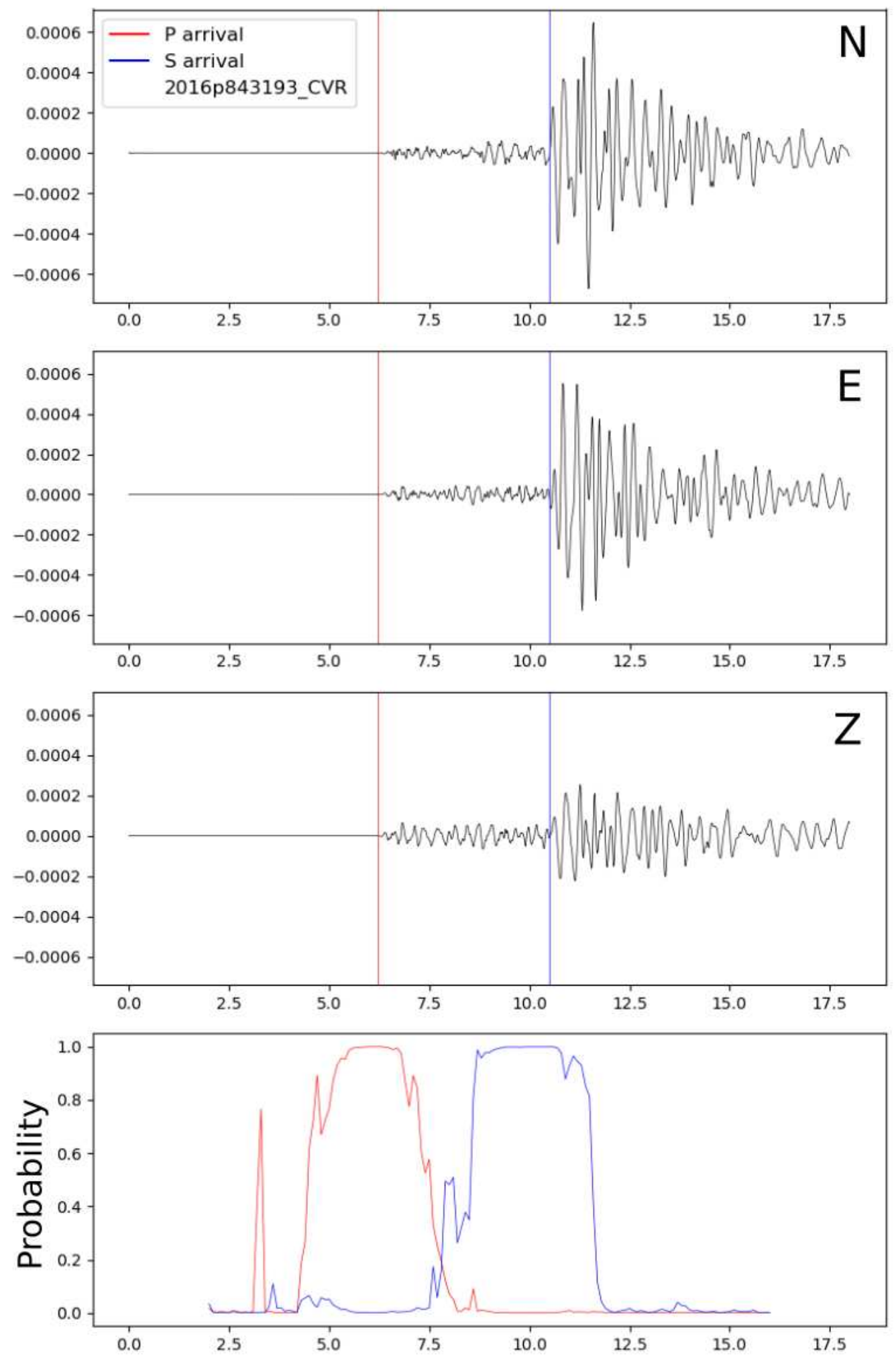

Figure A.3: Example of generalized phase detection GPD pick. The red and blue colors indicate the probability of $\mathrm{P}$ and $\mathrm{S}$ waves, respectively. The vertical bars are estimated picks. This is an example of a reliable $\mathrm{P}$ - and S-pick for station CVR. 


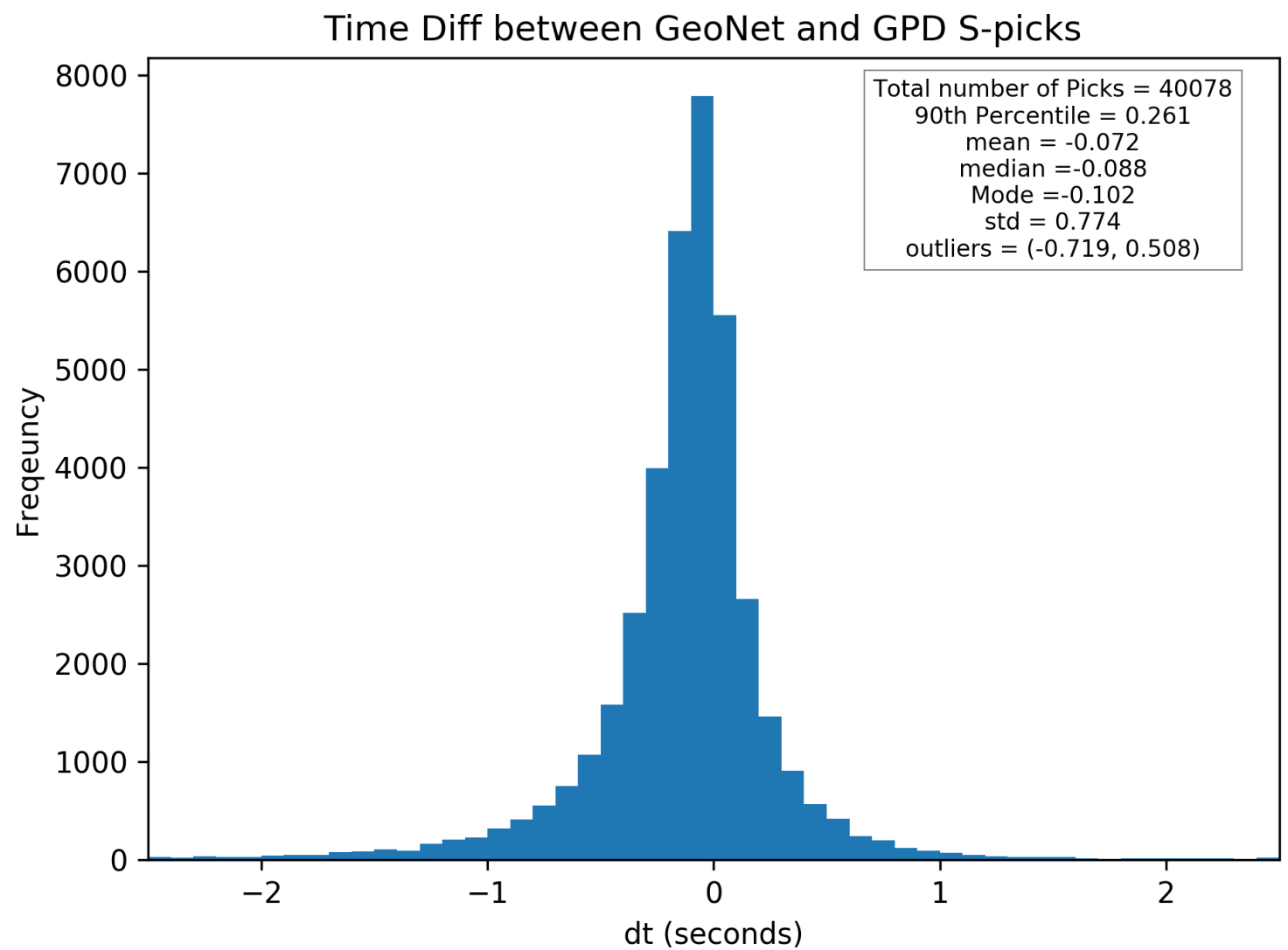

Figure A.4: Distributions of time difference between GeoNet manual S-picks and GDP S-picks. The distribution is normally distributed with mean -0.072 and standard deviation 0.774 


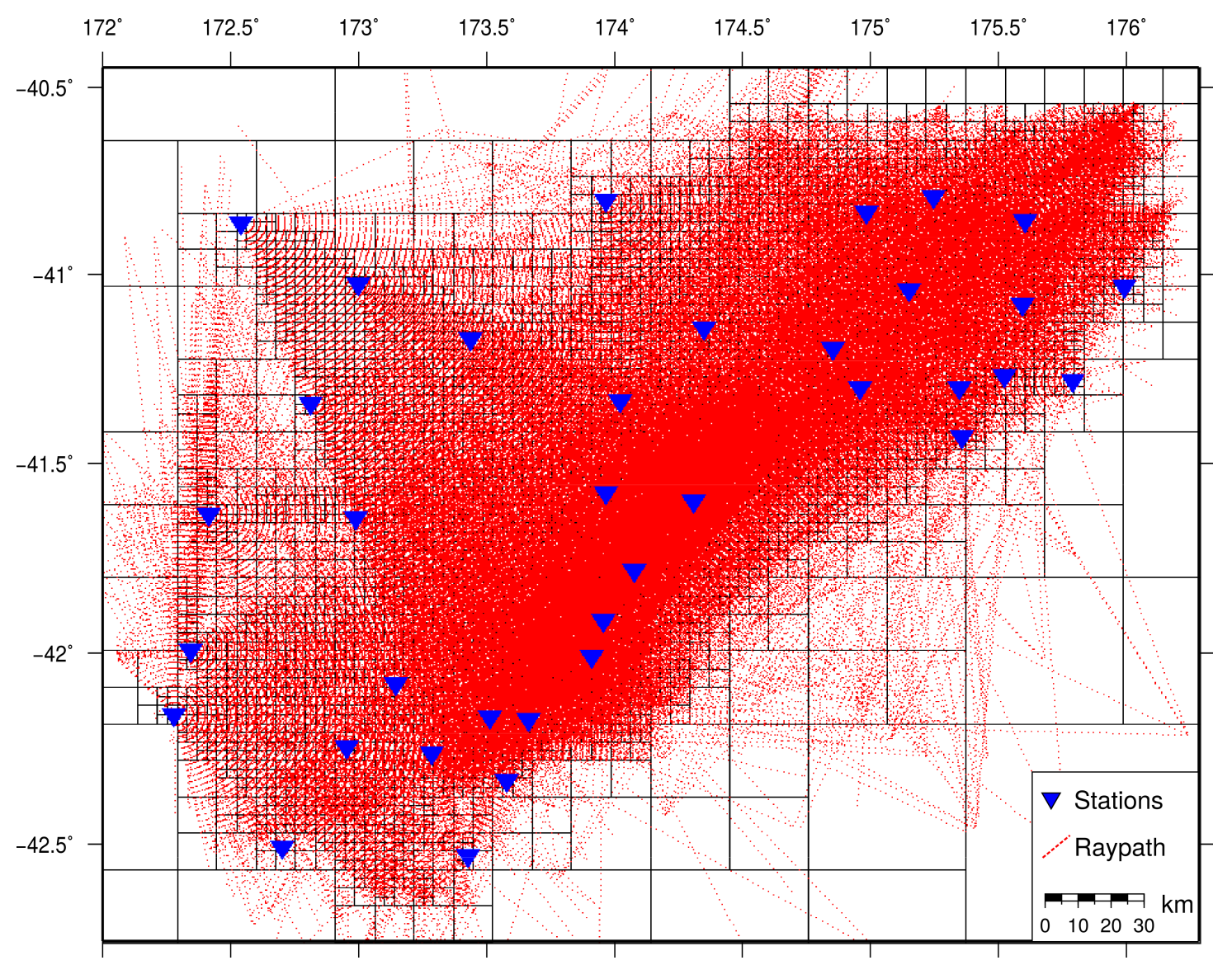

Figure A.5: Study area is divided into grid cell using quadtree gridding. Red lines show raypaths, and blue triangles are seismic stations. For each grid, a minimum of 20 and maximum of 80 raypaths pass through each grid cell, with a minimum grid size of $5 \times 5 \mathrm{~km}$ 


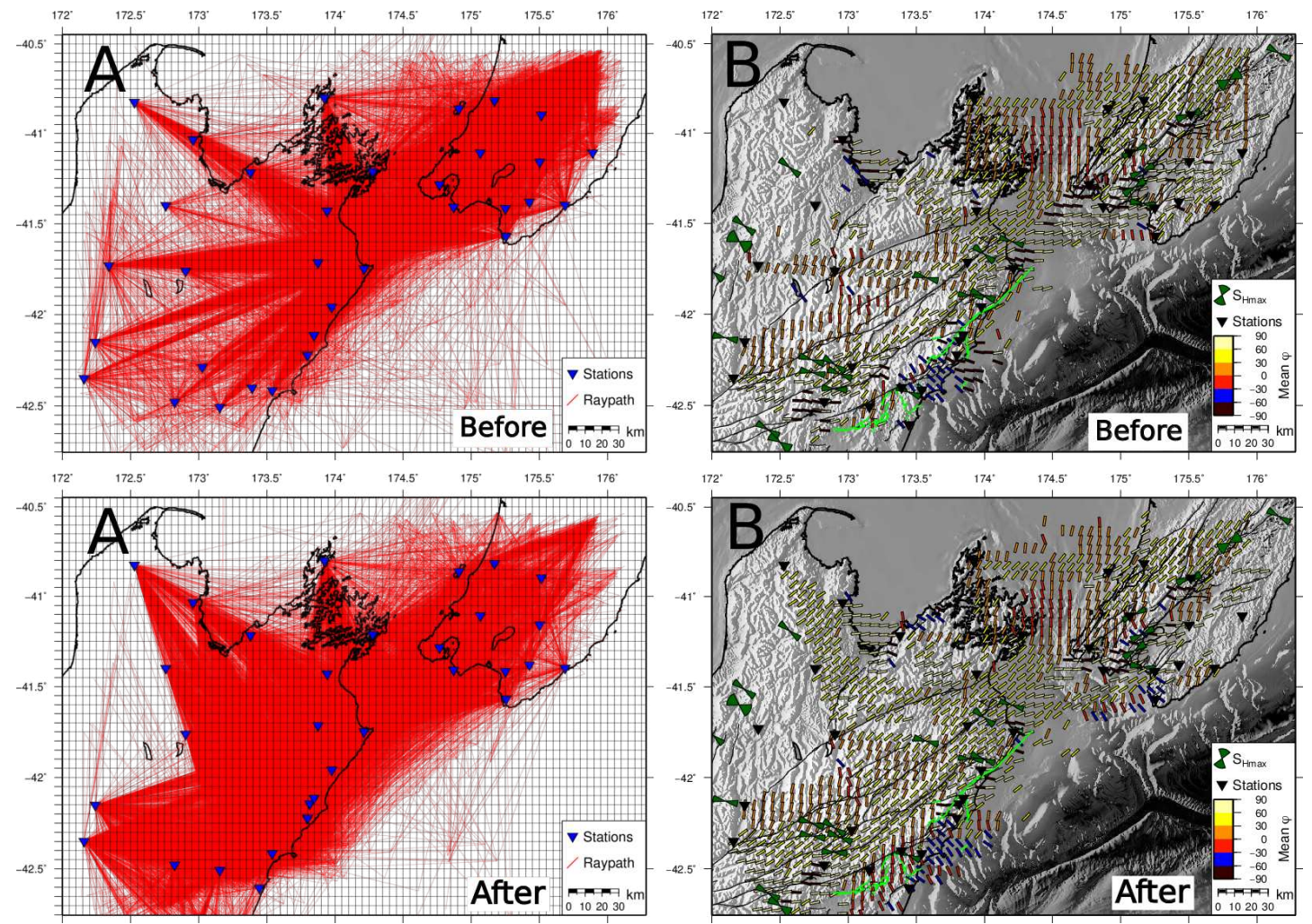

Figure A.6: Spatial averaging results of $\phi$ for before (top row) and after (bottom row) measurements. A: Study area is divided into grid cell using quadtree gridding. Red lines show raypaths, and blue inverted triangles are seismic stations. A minimum of 20 and maximum of 80 raypaths passing through each block, with a minimum block size of $5 \mathrm{~km}$ are the criteria used for gridding. B: Spatial averages with weighting inversely proportional to the square of the distance from the station. The coloured bars show the mean in each grid. Green line are the fault lines that ruptured during the Kaikōura main-shock. 

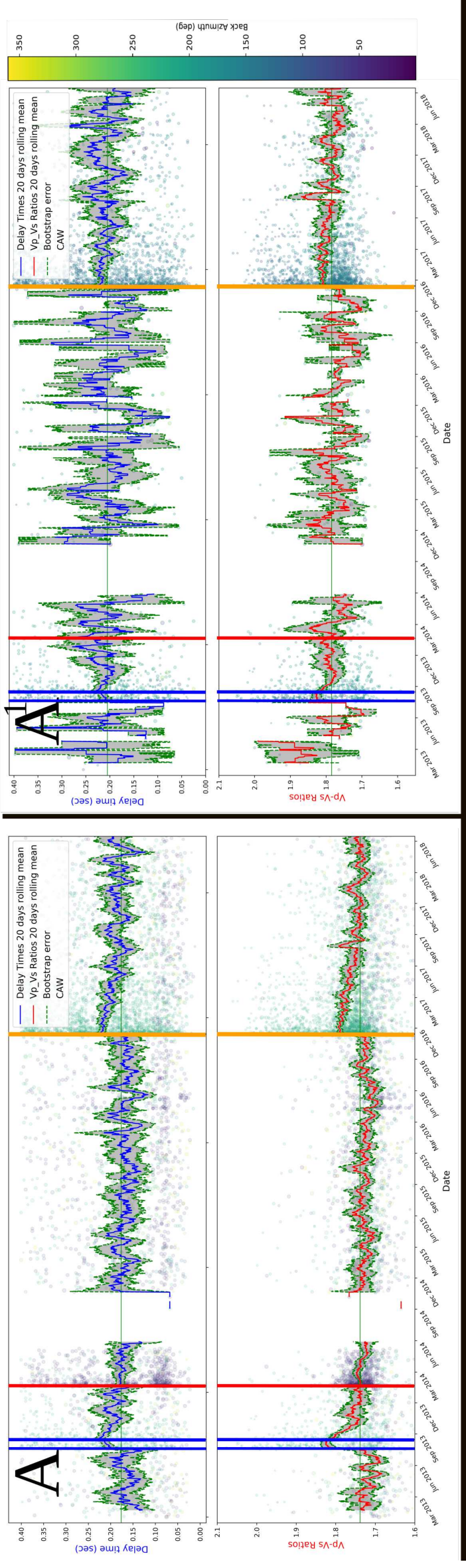

艺急芯

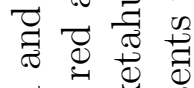

《)

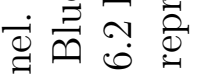

当当

वृ

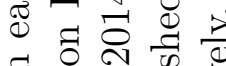

$\exists$.

专

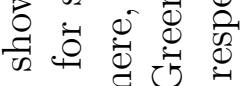

. $\frac{1}{2}$ 过

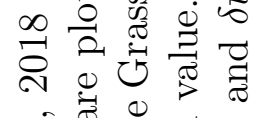

记

$-0.0$

๑

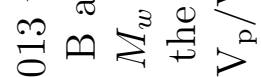

굘

悹晜

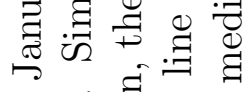

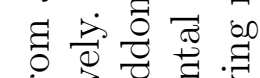

象.

龸

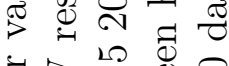

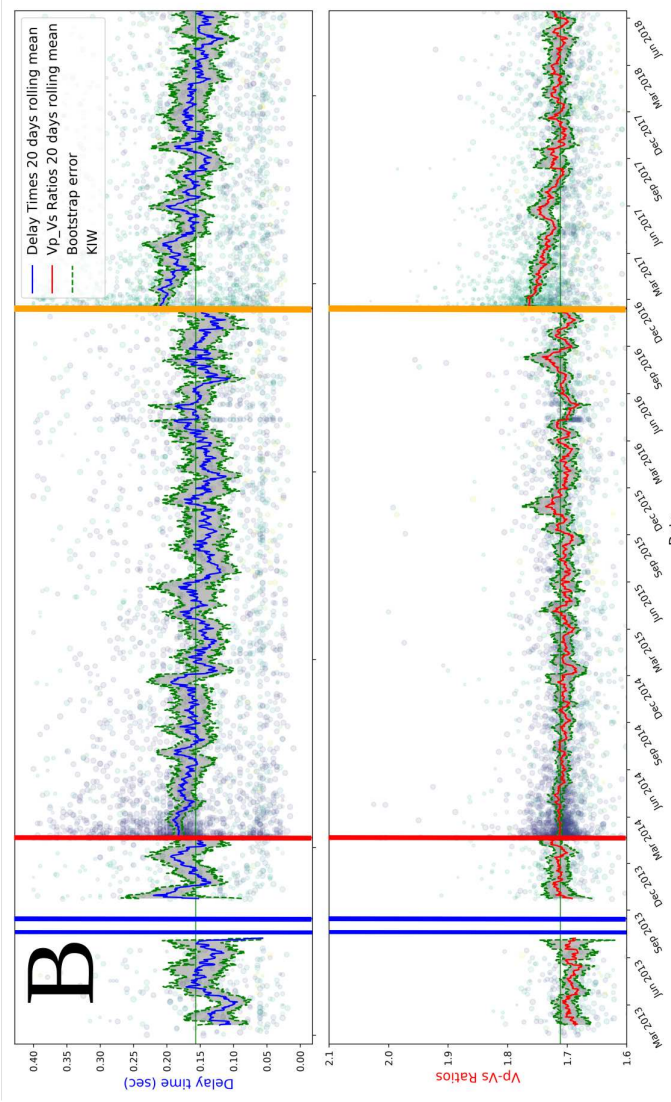

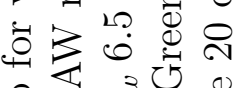

त्र

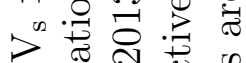

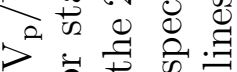

च

范

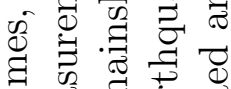

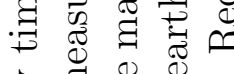

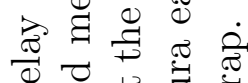

过

पै 步

की

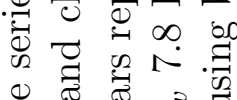

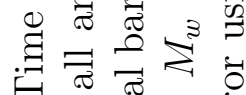

ㅂ.

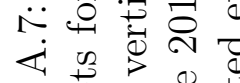

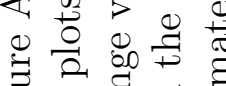

50 0 .
.

ซี 을 की 


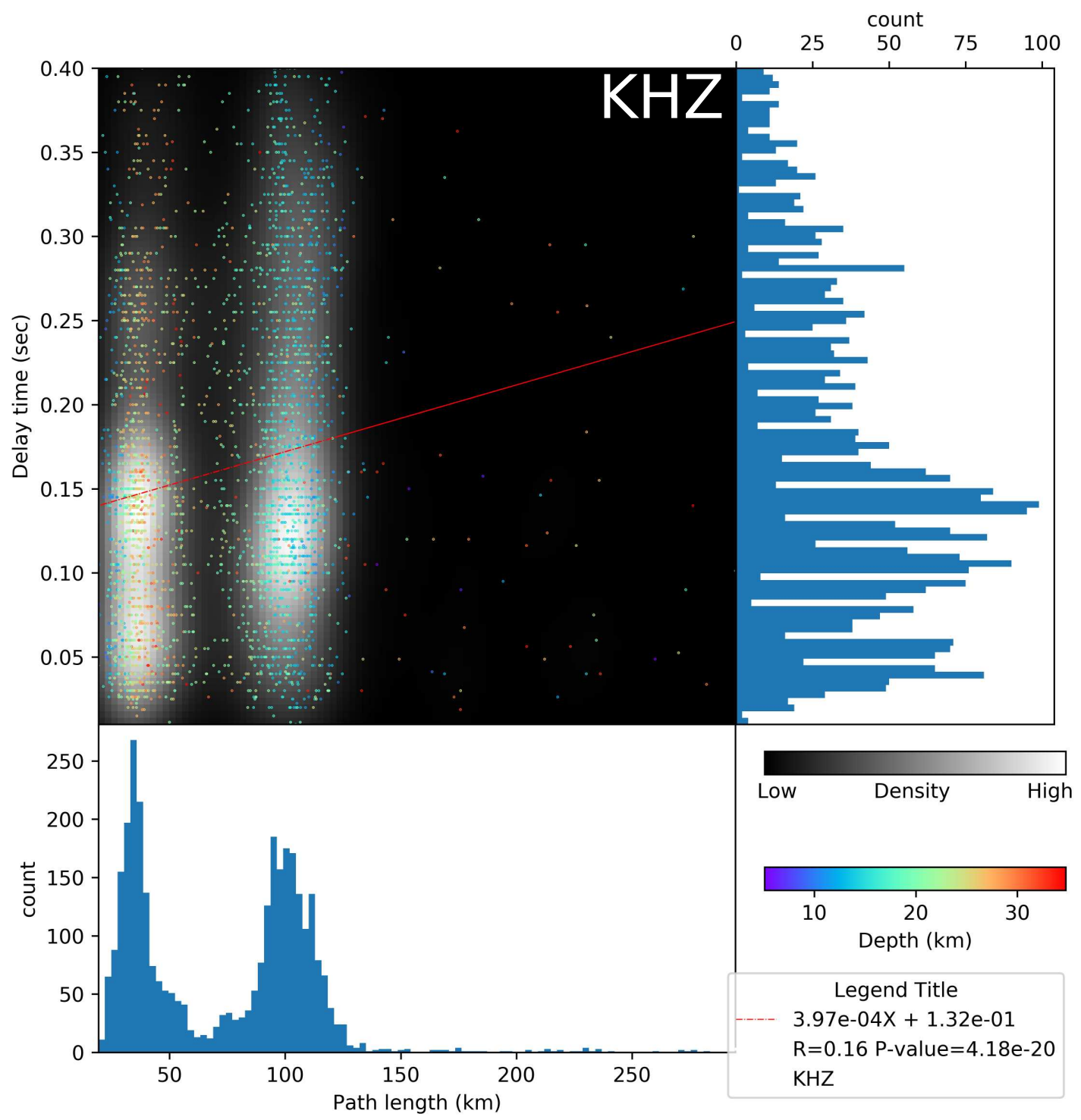

Figure A.8: Delay times versus path length for station KHZ. Distribution of the delay time and path length are plotted on the right and bottom respectively. The scattered points are coloured by the depths and plotted on a basemap of the kernel density (effectively heatmap). High density regions are shaded white with low regions black. The red line is the least square regression line with the equation and p-value displayed on the lower right corner. 


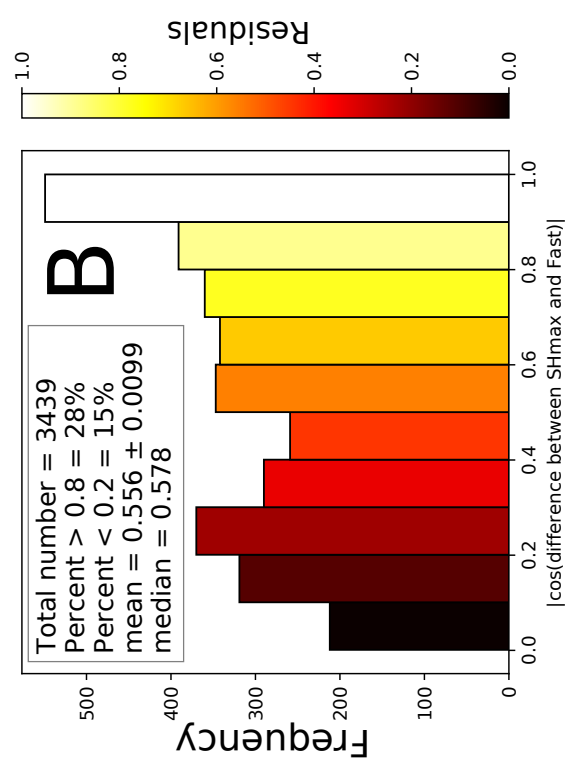

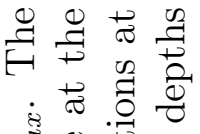

है $\frac{0}{\pi}$

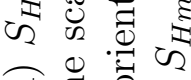

छ ㅎํ

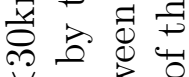

$\checkmark$ 范

पु

然

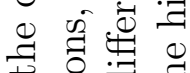

㟧荧

章索

. 녕

苟

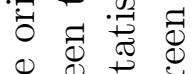

\&

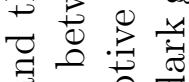

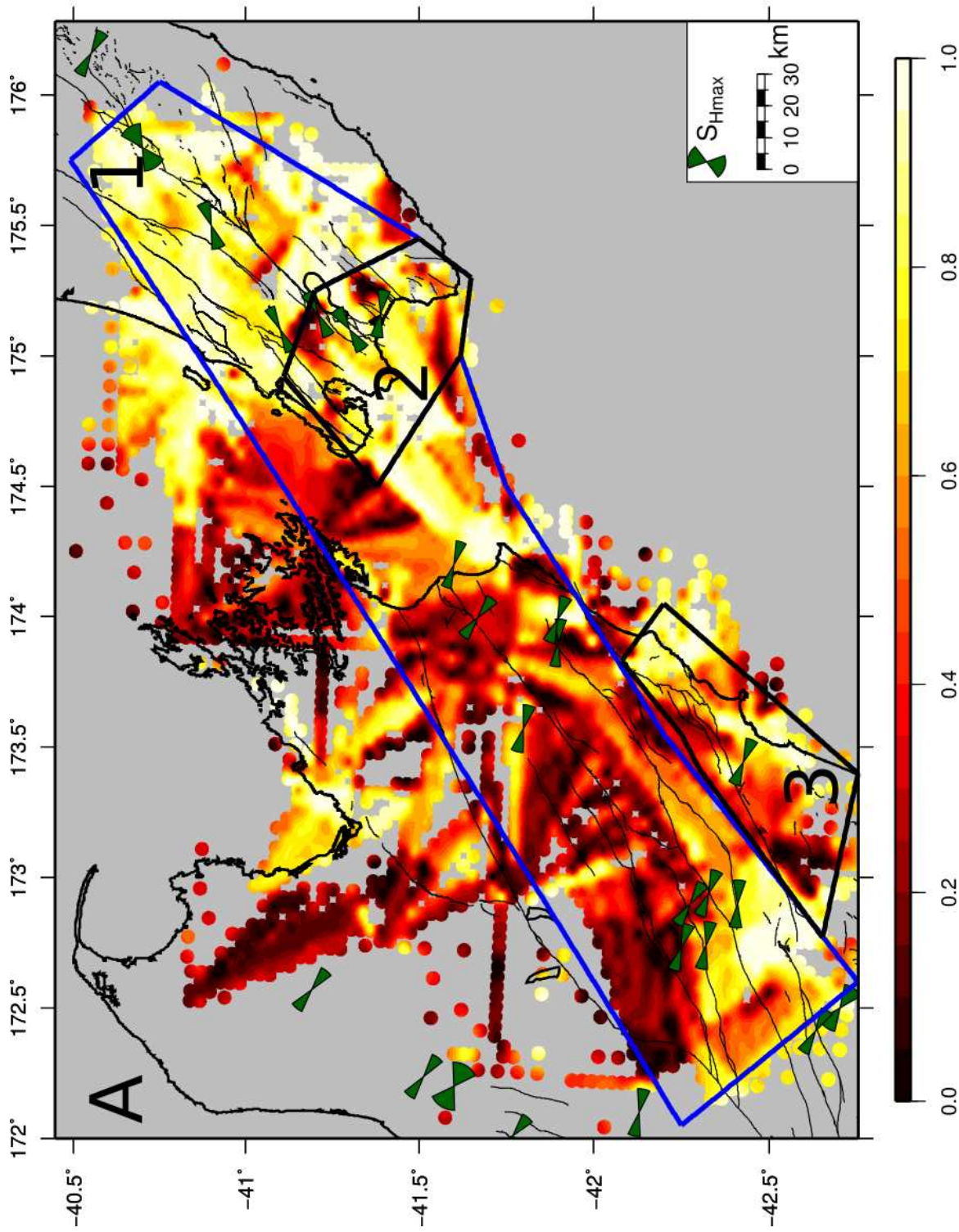

舟

율ㄹㄹ

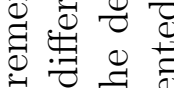

$\vec{G} E$

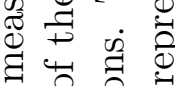

$\theta$ 莣

政

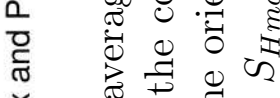

i.

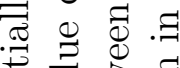

需

$\pi, 050$

$= \pm$

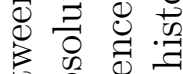

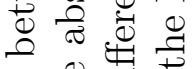

굥

总岕

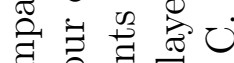

ఫี 웜 $\overrightarrow{0} . \exists$

( 000

.

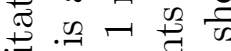

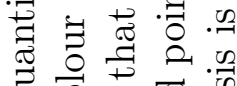

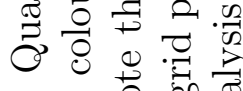

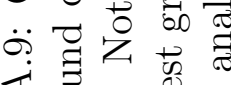

$<$ ठ

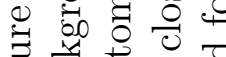

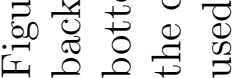




\section{B Supporting Information for Chapter 4 "Anisotropic Structure on a scale of tens of meters within Whataroa Valley in the vicinity of the Alpine Fault"}

\section{Introduction}

This section contains supplemental materials that augment reader's understanding of Chapter 4. It contains six tables and eight figures. 
Table B.1: Site-specific parameters used in EQcorrscans network trigger function (short-term average to long-term average, STA/LTA, detection algorithm. We used the vertical components for the triggering.

\begin{tabular}{llrrrrr}
\hline Station & sta_len $(\mathrm{s})$ & lat_len $(\mathrm{s})$ & lowcut $(\mathrm{Hz})$ & highcut $(\mathrm{Hz})$ & thres_on & thres_off \\
\hline WDSZ & 0.3 & 2 & 3 & 11 & 8 & 3 \\
WMSZ & 0.2 & 2 & 3 & 11 & 8 & 3 \\
WPSZ & 0.2 & 2 & 3 & 11 & 8 & 3 \\
WTSZ & 0.2 & 2 & 3 & 11 & 8 & 3 \\
WHAT2 & 0.3 & 3 & 3 & 12 & 8 & 3 \\
Reftek-VUW1 & 0.2 & 2 & 2 & 11 & 8 & 3 \\
Reftek-VUW2 & 0.2 & 2 & 2 & 11 & 8 & 3 \\
Reftek-VUW5 & 0.2 & 2 & 2 & 11 & 8 & 3 \\
C671 & 0.2 & 2 & 3 & 11 & 8 & 3 \\
C722 & 0.2 & 2 & 3 & 11 & 8 & 3 \\
C693 & 0.2 & 2 & 3 & 11 & 8 & 3 \\
C650 & 0.2 & 2 & 3 & 11 & 8 & 3 \\
\hline
\end{tabular}

Note: sta_len and lta_len are the lengths in samples of the short and long time average windows, respectively. lowcut and highcut define the low and high values for the bandpass filter applied respectively. thres_on and thres_off are the values that switch a single station trigger on and off, respectively.

Table B.2: Filters Applied to Waveforms Prior to MFAST analysis. Here we show the pair of band pass filters used in MFAST for both local and regional studies. The bold text are the filters used for this study. Generally lower band pass filters are used for regional studies compared to local studies. For local studies we often use microseismicity which are known to have higher frequencies compared to regional events, hence the need for higher frequencies.

\begin{tabular}{lllll}
\hline \multirow{2}{*}{ Filters } & \multicolumn{2}{c}{ Local studies } & \multicolumn{2}{c}{ Regional studies } \\
& low $\mathbf{( H z )}$ & high $\mathbf{( H z})$ & low $(\mathrm{Hz})$ & high $(\mathrm{Hz})$ \\
\hline Filter 1 & $\mathbf{1}$ & $\mathbf{5}$ & 0.4 & 4 \\
Filter 2 & $\mathbf{1}$ & $\mathbf{8}$ & 0.5 & 5 \\
Filter 3 & $\mathbf{1}$ & $\mathbf{1 5}$ & 0.2 & 3 \\
Filter 4 & $\mathbf{1}$ & $\mathbf{3 0}$ & 0.3 & 3 \\
Filter 5 & $\mathbf{3}$ & $\mathbf{5}$ & 0.5 & 4 \\
Filter 6 & $\mathbf{2}$ & $\mathbf{8}$ & 0.6 & 3 \\
Filter 7 & $\mathbf{3}$ & $\mathbf{1 5}$ & 0.8 & 6 \\
Filter 8 & $\mathbf{3}$ & $\mathbf{3 0}$ & 1 & 3 \\
Filter 9 & $\mathbf{5}$ & $\mathbf{1 0}$ & 1 & 5 \\
Filter 10 & $\mathbf{5}$ & $\mathbf{1 5}$ & 1 & 8 \\
Filter 11 & $\mathbf{5}$ & $\mathbf{3 0}$ & 2 & 3 \\
Filter 12 & $\mathbf{5}$ & $\mathbf{2 5}$ & 2 & 6 \\
Filter 13 & $\mathbf{1 0}$ & $\mathbf{2 0}$ & 3 & 8 \\
Filter 14 & $\mathbf{1 0}$ & $\mathbf{2 5}$ & 4 & 10 \\
\hline
\end{tabular}


Table B.3: Summary statistics of fast orientation $(\phi)$, delay time $(\delta t)$,percent anisotropy (\% Aniso) and crack density $(\epsilon)$ with their $95 \%$ confidence interval for stations used

\begin{tabular}{|c|c|c|c|c|}
\hline Station & Mean $\phi\left(^{\circ}\right)$ & Mean $\delta t(\mathrm{~s})$ & Mean \%Aniso & Mean $\epsilon$ \\
\hline C609 & $124.876 \pm-41.982$ & $0.04 \pm 0.002$ & $0.976 \pm 0.074$ & $0.036 \pm 0.016$ \\
\hline $\mathrm{C} 621$ & $39.164 \pm-23.494$ & $0.08 \pm 0.054$ & $2.058 \pm 1.379$ & $0.057 \pm 0.03$ \\
\hline $\mathrm{C} 622$ & $63.375 \pm-18.546$ & $0.092 \pm 0.017$ & $2.078 \pm 0.338$ & $0.081 \pm 0.044$ \\
\hline C623 & $117.556 \pm-40.516$ & $0.032 \pm 0.015$ & $0.792 \pm 0.369$ & $0.018 \pm 0.012$ \\
\hline C624 & $45.229 \pm-23.888$ & $0.055 \pm 0.013$ & $1.389 \pm 0.361$ & $0.042 \pm 0.02$ \\
\hline C625 & $87.679 \pm-64.765$ & $0.087 \pm 0.033$ & $2.214 \pm 0.708$ & $0.064 \pm 0.027$ \\
\hline C626 & $22.227 \pm-12.088$ & $0.11 \pm 0.024$ & $3.124 \pm 1.401$ & $0.1 \pm 0.055$ \\
\hline $\mathrm{C} 627$ & $124.758 \pm-28.296$ & $0.066 \pm 0.042$ & $1.569 \pm 0.892$ & $0.037 \pm 0.011$ \\
\hline C628 & $70.0 \pm 0.000$ & $0.12 \pm 0.000$ & $2.752 \pm 0.000$ & $0.078 \pm 0.000$ \\
\hline C629 & $173.558 \pm-12.237$ & $0.132 \pm 0.031$ & $3.028 \pm 0.518$ & $0.117 \pm 0.071$ \\
\hline C630 & $43.389 \pm-11.975$ & $0.048 \pm 0.017$ & $1.178 \pm 0.398$ & $0.035 \pm 0.015$ \\
\hline C631 & $60.258 \pm-9.872$ & $0.08 \pm 0.022$ & $1.773 \pm 0.497$ & $0.069 \pm 0.051$ \\
\hline C632 & $111.036 \pm-46.773$ & $0.065 \pm 0.036$ & $1.57 \pm 0.89$ & $0.035 \pm 0.018$ \\
\hline C633 & $60.825 \pm-25.588$ & $0.081 \pm 0.041$ & $1.976 \pm 0.867$ & $0.056 \pm 0.024$ \\
\hline C634 & $30.469 \pm-29.147$ & $0.133 \pm 0.023$ & $3.072 \pm 0.484$ & $0.112 \pm 0.063$ \\
\hline C635 & $94.624 \pm-13.458$ & $0.062 \pm 0.027$ & $1.46 \pm 0.645$ & $0.036 \pm 0.021$ \\
\hline C636 & $64.851 \pm-31.357$ & $0.048 \pm 0.017$ & $1.258 \pm 0.555$ & $0.03 \pm 0.011$ \\
\hline C637 & $160.00 \pm 0.000$ & $0.1 \pm 0.00$ & $3.401 \pm 0.00$ & $0.054 \pm 0.00$ \\
\hline C638 & $167.583 \pm-28.213$ & $0.04 \pm 0.019$ & $1.032 \pm 0.468$ & $0.024 \pm 0.014$ \\
\hline C640 & $23 \pm-25.68$ & $0.038 \pm 0.028$ & $0.943 \pm 0.717$ & $0.045 \pm 0.046$ \\
\hline C641 & $138.919 \pm-38.403$ & $0.033 \pm 0.013$ & $0.79 \pm 0.325$ & $0.019 \pm 0.008$ \\
\hline C642 & $52.993 \pm-42.748$ & $0.022 \pm 0.007$ & $0.516 \pm 0.167$ & $0.014 \pm 0.005$ \\
\hline $\mathrm{C} 643$ & $12 \pm 0.000$ & $0.056 \pm 0.00$ & $1.381 \pm 0.00$ & $0.038 \pm 0.00$ \\
\hline C644 & $50.943 \pm-32.769$ & $0.084 \pm 0.055$ & $2.019 \pm 1.26$ & $0.069 \pm 0.042$ \\
\hline C645 & $51.997 \pm-2.83$ & $0.06 \pm 0.032$ & $1.66 \pm 0.997$ & $0.068 \pm 0.063$ \\
\hline C646 & $64 \pm 0.000$ & $0.06 \pm 0.00$ & $1.385 \pm 0.00$ & $0.041 \pm 0.00$ \\
\hline $\mathrm{C} 647$ & $90.0 \pm 247.866$ & $0.061 \pm 0.061$ & $1.455 \pm 1.418$ & $0.025 \pm 0.019$ \\
\hline C648 & $154 \pm 0.000$ & $0.037 \pm 0.00$ & $0.897 \pm 0.00$ & $0.022 \pm 0.00$ \\
\hline C649 & $57.86 \pm-27.851$ & $0.066 \pm 0.023$ & $1.503 \pm 0.499$ & $0.038 \pm 0.011$ \\
\hline C651 & $9.565 \pm-37.428$ & $0.112 \pm 0.071$ & $2.577 \pm 1.652$ & $0.05 \pm 0.017$ \\
\hline C652 & $92.409 \pm-40.331$ & $0.087 \pm 0.041$ & $2.126 \pm 0.937$ & $0.049 \pm 0.027$ \\
\hline C653 & $10.042 \pm-46.047$ & $0.036 \pm 0.02$ & $0.864 \pm 0.455$ & $0.028 \pm 0.023$ \\
\hline C654 & $48.923 \pm-12.937$ & $0.061 \pm 0.001$ & $1.391 \pm 0.054$ & $0.042 \pm 0.018$ \\
\hline C655 & $44.196 \pm-5.874$ & $0.025 \pm 0.013$ & $0.563 \pm 0.276$ & $0.016 \pm 0.009$ \\
\hline C656 & $81.5 \pm-63.286$ & $0.116 \pm 0.078$ & $2.764 \pm 1.369$ & $0.073 \pm 0.059$ \\
\hline C657 & $42.298 \pm-31.578$ & $0.055 \pm 0.024$ & $1.274 \pm 0.53$ & $0.042 \pm 0.021$ \\
\hline C658 & $74.034 \pm-12.025$ & $0.085 \pm 0.059$ & $2.021 \pm 1.421$ & $0.071 \pm 0.07$ \\
\hline C660 & $30.749 \pm-1.921$ & $0.138 \pm 0.068$ & $3.485 \pm 1.83$ & $0.109 \pm 0.072$ \\
\hline C661 & $136.228 \pm-42.903$ & $0.069 \pm 0.021$ & $1.602 \pm 0.501$ & $0.041 \pm 0.014$ \\
\hline C662 & $34.99 \pm-4.327$ & $0.083 \pm 0.041$ & $2.186 \pm 1.399$ & $0.058 \pm 0.021$ \\
\hline
\end{tabular}

Only high quality measurements (described in Section 3.3.2) are presented in the summary table. \pm represents the $95 \%$ confidence intervals. Station averages and standard deviation for fast orientations were performed using circular statistics. 
Table B.4: Continuation of Table B.3

\begin{tabular}{|c|c|c|c|c|}
\hline Station & Mean $\phi\left(^{\circ}\right)$ & Mean $\delta t(\mathrm{~s})$ & Mean \%Aniso & Mean $\epsilon$ \\
\hline C663 & $127 \pm 0.000$ & $0.085 \pm 0.00$ & $2.113 \pm 0.00$ & $0.1 \pm 0.00$ \\
\hline C664 & $68.5 \pm-22.661$ & $0.084 \pm 0.071$ & $1.82 \pm 1.517$ & $0.052 \pm 0.027$ \\
\hline C665 & $33.227 \pm-7.21$ & $0.094 \pm 0.024$ & $2.075 \pm 0.5$ & $0.053 \pm 0.009$ \\
\hline C666 & $46.037 \pm-27.602$ & $0.049 \pm 0.035$ & $1.065 \pm 0.751$ & $0.031 \pm 0.021$ \\
\hline C667 & $35.025 \pm-44.191$ & $0.122 \pm 0.033$ & $2.747 \pm 0.713$ & $0.068 \pm 0.018$ \\
\hline C668 & $133.488 \pm-9.174$ & $0.072 \pm 0.012$ & $1.609 \pm 0.307$ & $0.041 \pm 0.012$ \\
\hline C669 & $69.587 \pm-37.98$ & $0.058 \pm 0.035$ & $1.386 \pm 0.874$ & $0.032 \pm 0.019$ \\
\hline C670 & $16.753 \pm-46.522$ & $0.11 \pm 0.033$ & $2.517 \pm 0.732$ & $0.069 \pm 0.036$ \\
\hline C671 & $178.23 \pm-29.584$ & $0.067 \pm 0.04$ & $1.597 \pm 0.942$ & $0.047 \pm 0.039$ \\
\hline C672 & $137.332 \pm-13.533$ & $0.041 \pm 0.014$ & $0.944 \pm 0.33$ & $0.022 \pm 0.004$ \\
\hline C673 & $57.109 \pm-23.951$ & $0.078 \pm 0.047$ & $1.684 \pm 0.989$ & $0.043 \pm 0.024$ \\
\hline C674 & $12.569 \pm-10.705$ & $0.079 \pm 0.021$ & $1.769 \pm 0.439$ & $0.053 \pm 0.019$ \\
\hline C675 & $67.854 \pm-13.553$ & $0.059 \pm 0.001$ & $1.315 \pm 0.041$ & $0.041 \pm 0.011$ \\
\hline C676 & $54.811 \pm-16.198$ & $0.092 \pm 0.043$ & $2.064 \pm 0.916$ & $0.062 \pm 0.024$ \\
\hline C677 & $63 \pm 0.000$ & $0.06 \pm 0.00$ & $1.437 \pm 0.00$ & $0.04 \pm 0.00$ \\
\hline C678 & $147 \pm 0.000$ & $0.022 \pm 0.00$ & $0.541 \pm 0.00$ & $0.025 \pm 0.00$ \\
\hline C679 & $145.859 \pm-29.649$ & $0.135 \pm 0.042$ & $3.084 \pm 0.942$ & $0.171 \pm 0.158$ \\
\hline C681 & $22.651 \pm-7.873$ & $0.092 \pm 0.047$ & $2.105 \pm 0.992$ & $0.062 \pm 0.033$ \\
\hline C682 & $14.199 \pm-5.164$ & $0.04 \pm 0.001$ & $0.993 \pm 0.131$ & $0.046 \pm 0.035$ \\
\hline C683 & $47.759 \pm-33.856$ & $0.053 \pm 0.007$ & $1.345 \pm 0.256$ & $0.057 \pm 0.054$ \\
\hline C684 & $70.768 \pm-21.625$ & $0.073 \pm 0.028$ & $1.723 \pm 0.639$ & $0.09 \pm 0.083$ \\
\hline C685 & $132 \pm 0.000$ & $0.02 \pm 0.00$ & $0.478 \pm 0.00$ & $0.012 \pm 0.00$ \\
\hline C687 & $57.498 \pm-4.16$ & $0.066 \pm 0.027$ & $1.511 \pm 0.612$ & $0.085 \pm 0.081$ \\
\hline C688 & $62 \pm-40.145$ & $0.051 \pm 0.04$ & $1.164 \pm 0.896$ & $0.039 \pm 0.035$ \\
\hline C689 & $31.595 \pm-15.819$ & $0.059 \pm 0.045$ & $1.457 \pm 0.997$ & $0.036 \pm 0.029$ \\
\hline C690 & $12.25 \pm-39.687$ & $0.114 \pm 0.044$ & $2.621 \pm 0.997$ & $0.117 \pm 0.057$ \\
\hline C691 & $25.632 \pm-25.248$ & $0.066 \pm 0.011$ & $1.75 \pm 0.318$ & $0.04 \pm 0.01$ \\
\hline C693 & $13.432 \pm-42.2$ & $0.082 \pm 0.073$ & $1.881 \pm 1.654$ & $0.064 \pm 0.065$ \\
\hline C695 & $13 \pm 0.000$ & $0.08 \pm 0.00$ & $1.863 \pm 0.00$ & $0.052 \pm 0.00$ \\
\hline C697 & $61.196 \pm-4.45$ & $0.08 \pm 0.001$ & $1.874 \pm 0.215$ & $0.068 \pm 0.036$ \\
\hline C698 & $56.25 \pm-1.479$ & $0.061 \pm 0.001$ & $1.388 \pm 0.037$ & $0.058 \pm 0.032$ \\
\hline C699 & $149 \pm-5.013$ & $0.027 \pm 0.001$ & $0.681 \pm 0.002$ & $0.046 \pm 0.039$ \\
\hline $\mathrm{C} 700$ & $27.995 \pm-20.952$ & $0.142 \pm 0.027$ & $3.29 \pm 0.592$ & $0.131 \pm 0.086$ \\
\hline C701 & $38 \pm 0.000$ & $0.098 \pm 0.00$ & $2.222 \pm 0.00$ & $0.064 \pm 0.00$ \\
\hline C702 & $61.571 \pm-14.384$ & $0.05 \pm 0.013$ & $1.192 \pm 0.284$ & $0.038 \pm 0.017$ \\
\hline C703 & $153.5 \pm-4.509$ & $0.032 \pm 0.016$ & $0.863 \pm 0.289$ & $0.019 \pm 0.012$ \\
\hline C704 & $12.5 \pm-67.362$ & $0.056 \pm 0.004$ & $1.451 \pm 0.264$ & $0.058 \pm 0.044$ \\
\hline C706 & $56.138 \pm-20.01$ & $0.088 \pm 0.06$ & $2.469 \pm 1.911$ & $0.097 \pm 0.106$ \\
\hline C707 & $150.911 \pm-22.789$ & $0.072 \pm 0.028$ & $1.917 \pm 0.975$ & $0.058 \pm 0.015$ \\
\hline C708 & $176.282 \pm-10.407$ & $0.038 \pm 0.002$ & $0.912 \pm 0.112$ & $0.032 \pm 0.016$ \\
\hline C709 & $34.961 \pm-6.071$ & $0.084 \pm 0.006$ & $2.07 \pm 0.179$ & $0.063 \pm 0.021$ \\
\hline C714 & $173.018 \pm-37.664$ & $0.099 \pm 0.021$ & $2.381 \pm 0.538$ & $0.058 \pm 0.018$ \\
\hline C715 & $80.991 \pm-10.912$ & $0.121 \pm 0.026$ & $3.042 \pm 0.76$ & $0.108 \pm 0.085$ \\
\hline C716 & $9.393 \pm-29.617$ & $0.092 \pm 0.029$ & $2.263 \pm 0.732$ & $0.072 \pm 0.034$ \\
\hline $\mathrm{C} 717$ & $85.45 \pm-17.857$ & $0.023 \pm 0.012$ & $0.515 \pm 0.257$ & $0.015 \pm 0.007$ \\
\hline
\end{tabular}


Table B.5: Continuation of Table B.4

\begin{tabular}{|c|c|c|c|c|}
\hline Station & Mean $\phi\left(^{\circ}\right)$ & Mean $\delta t(\mathrm{~s})$ & Mean \%Aniso & Mean $\epsilon$ \\
\hline C718 & $24.805 \pm-9.419$ & $0.093 \pm 0.04$ & $2.353 \pm 1.015$ & $0.075 \pm 0.047$ \\
\hline C719 & $117.915 \pm-37.923$ & $0.077 \pm 0.017$ & $1.815 \pm 0.431$ & $0.063 \pm 0.041$ \\
\hline $\mathrm{C} 720$ & $71.5 \pm-3.504$ & $0.018 \pm 0.003$ & $0.387 \pm 0.067$ & $0.011 \pm 0.004$ \\
\hline $\mathrm{C} 721$ & $79 \pm 0.000$ & $0.165 \pm 0.00$ & $3.791 \pm 0.00$ & $0.242 \pm 0.00$ \\
\hline $\mathrm{C} 722$ & $54.306 \pm-51.711$ & $0.046 \pm 0.021$ & $1.005 \pm 0.45$ & $0.025 \pm 0.013$ \\
\hline $\mathrm{C} 723$ & $72.622 \pm-11.933$ & $0.054 \pm 0.01$ & $1.313 \pm 0.28$ & $0.044 \pm 0.022$ \\
\hline $\mathrm{C} 724$ & $14.807 \pm-21.263$ & $0.076 \pm 0.025$ & $1.785 \pm 0.554$ & $0.054 \pm 0.023$ \\
\hline $\mathrm{C} 725$ & $34 \pm-4.007$ & $0.186 \pm 0.009$ & $4.794 \pm 0.796$ & $0.103 \pm 0.016$ \\
\hline $\mathrm{C} 727$ & $110.744 \pm-50.654$ & $0.123 \pm 0.031$ & $2.877 \pm 0.826$ & $0.09 \pm 0.049$ \\
\hline $\mathrm{C} 728$ & $33.298 \pm-24.456$ & $0.066 \pm 0.029$ & $1.574 \pm 0.645$ & $0.048 \pm 0.025$ \\
\hline C729 & $62.859 \pm-42.009$ & $0.099 \pm 0.054$ & $2.611 \pm 1.782$ & $0.056 \pm 0.026$ \\
\hline $\mathrm{C} 730$ & $91.897 \pm-39.872$ & $0.06 \pm 0.038$ & $1.377 \pm 0.82$ & $0.034 \pm 0.022$ \\
\hline C731 & $34.982 \pm-16.226$ & $0.139 \pm 0.047$ & $3.154 \pm 0.991$ & $0.074 \pm 0.024$ \\
\hline $\mathrm{C} 733$ & $47.994 \pm-5.208$ & $0.018 \pm 0.001$ & $0.416 \pm 0.017$ & $0.01 \pm 0.002$ \\
\hline $\mathrm{C} 734$ & $56.173 \pm-5.055$ & $0.017 \pm 0.001$ & $0.373 \pm 0.024$ & $0.01 \pm 0.003$ \\
\hline $\mathrm{C} 735$ & $100.725 \pm-52.205$ & $0.105 \pm 0.036$ & $2.332 \pm 0.751$ & $0.054 \pm 0.02$ \\
\hline $\mathrm{C} 736$ & $77 \pm-30.888$ & $0.06 \pm 0.00$ & $1.389 \pm 0.023$ & $0.051 \pm 0.018$ \\
\hline $\mathrm{C} 737$ & $34.123 \pm-7.773$ & $0.019 \pm 0.001$ & $0.466 \pm 0.042$ & $0.012 \pm 0.004$ \\
\hline $\mathrm{C} 738$ & $62.559 \pm-12.796$ & $0.018 \pm 0.001$ & $0.411 \pm 0.037$ & $0.012 \pm 0.004$ \\
\hline C739 & $21.845 \pm-10.847$ & $0.073 \pm 0.028$ & $1.629 \pm 0.588$ & $0.045 \pm 0.015$ \\
\hline $\mathrm{C} 743$ & $40.743 \pm-17.296$ & $0.03 \pm 0.017$ & $0.706 \pm 0.367$ & $0.017 \pm 0.009$ \\
\hline $\mathrm{C} 746$ & $100.815 \pm-18.976$ & $0.019 \pm 0.002$ & $0.442 \pm 0.048$ & $0.01 \pm 0.002$ \\
\hline $\mathrm{C} 747$ & $159.947 \pm-35.961$ & $0.089 \pm 0.056$ & $2.222 \pm 1.424$ & $0.045 \pm 0.031$ \\
\hline C749 & $64.472 \pm-17.94$ & $0.058 \pm 0.049$ & $1.269 \pm 1.065$ & $0.028 \pm 0.012$ \\
\hline $\mathrm{C} 752$ & $177 \pm 0.000$ & $0.14 \pm 0.00$ & $3.328 \pm 0.00$ & $0.091 \pm 0.00$ \\
\hline $\mathrm{C} 754$ & $51.5 \pm-27.571$ & $0.054 \pm 0.023$ & $1.428 \pm 0.32$ & $0.032 \pm 0.018$ \\
\hline $\mathrm{C} 756$ & $74.389 \pm-27.72$ & $0.056 \pm 0.031$ & $1.248 \pm 0.682$ & $0.032 \pm 0.024$ \\
\hline $\mathrm{C} 757$ & $78.5 \pm-0.5$ & $0.105 \pm 0.024$ & $2.414 \pm 0.625$ & $0.154 \pm 0.139$ \\
\hline $\mathrm{C} 758$ & $73 \pm 0.000$ & $0.089 \pm 0.00$ & $2.156 \pm 0.00$ & $0.06 \pm 0.00$ \\
\hline $\mathrm{C} 761$ & $67.421 \pm-28.195$ & $0.029 \pm 0.023$ & $0.702 \pm 0.543$ & $0.022 \pm 0.013$ \\
\hline $\mathrm{C} 763$ & $6.5 \pm-11.66$ & $0.049 \pm 0.011$ & $1.18 \pm 0.235$ & $0.07 \pm 0.061$ \\
\hline $\mathrm{C} 780$ & $29 \pm 0.000$ & $0.055 \pm 0.00$ & $1.264 \pm 0.00$ & $0.034 \pm 0.00$ \\
\hline $\mathrm{C} 783$ & $83.822 \pm-27.61$ & $0.087 \pm 0.014$ & $2.229 \pm 0.378$ & $0.079 \pm 0.052$ \\
\hline $\mathrm{C} 784$ & $57.633 \pm-10.984$ & $0.03 \pm 0.021$ & $0.862 \pm 0.757$ & $0.021 \pm 0.011$ \\
\hline $\mathrm{C} 785$ & $78.5 \pm-20.343$ & $0.074 \pm 0.019$ & $1.743 \pm 0.53$ & $0.034 \pm 0.005$ \\
\hline $\mathrm{C} 787$ & $90.376 \pm-41.962$ & $0.024 \pm 0.011$ & $0.547 \pm 0.248$ & $0.018 \pm 0.015$ \\
\hline C788 & $25.2 \pm-1.47$ & $0.153 \pm 0.002$ & $3.557 \pm 0.358$ & $0.098 \pm 0.042$ \\
\hline C789 & $81.012 \pm-9.809$ & $0.067 \pm 0.004$ & $1.613 \pm 0.263$ & $0.036 \pm 0.01$ \\
\hline C794 & $60.177 \pm-20.958$ & $0.078 \pm 0.021$ & $1.727 \pm 0.404$ & $0.052 \pm 0.012$ \\
\hline $\mathrm{C} 814$ & $26 \pm-3.003$ & $0.06 \pm 0.055$ & $1.348 \pm 1.186$ & $0.038 \pm 0.036$ \\
\hline C839 & $39.591 \pm-12.03$ & $0.084 \pm 0.08$ & $1.957 \pm 1.613$ & $0.05 \pm 0.051$ \\
\hline $\mathrm{C} 840$ & $69.303 \pm-34.044$ & $0.109 \pm 0.037$ & $2.325 \pm 0.727$ & $0.062 \pm 0.027$ \\
\hline $\mathrm{C} 842$ & $52.377 \pm-31.55$ & $0.073 \pm 0.052$ & $1.952 \pm 1.417$ & $0.053 \pm 0.053$ \\
\hline C843 & $58 \pm 0.000$ & $0.06 \pm 0.00$ & $1.256 \pm 0.00$ & $0.029 \pm 0.00$ \\
\hline $\mathrm{C} 844$ & $89.225 \pm-43.312$ & $0.075 \pm 0.044$ & $1.758 \pm 0.969$ & $0.053 \pm 0.047$ \\
\hline
\end{tabular}


Table B.6: Continuation of Table B.5

\begin{tabular}{lllll}
\hline Station & Mean $\phi\left(^{\circ}\right)$ & Mean $\delta t(\mathrm{~s})$ & Mean \%Aniso & Mean $\epsilon$ \\
\hline C845 & $152.582 \pm-52.278$ & $0.058 \pm 0.024$ & $1.308 \pm 0.501$ & $0.036 \pm 0.01$ \\
C846 & $13.012 \pm-28.605$ & $0.044 \pm 0.017$ & $0.975 \pm 0.37$ & $0.028 \pm 0.017$ \\
C847 & $7.746 \pm-45.831$ & $0.044 \pm 0.019$ & $1.16 \pm 0.605$ & $0.028 \pm 0.017$ \\
C848 & $104.179 \pm-22.647$ & $0.079 \pm 0.026$ & $1.735 \pm 0.559$ & $0.059 \pm 0.039$ \\
C849 & $54 \pm 0.000$ & $0.009 \pm 0.00$ & $0.21 \pm 0.00$ & $0.006 \pm 0.00$ \\
C850 & $117 \pm-26.933$ & $0.029 \pm 0.012$ & $0.68 \pm 0.299$ & $0.041 \pm 0.039$ \\
C851 & $64.224 \pm-12.386$ & $0.117 \pm 0.058$ & $3.173 \pm 1.85$ & $0.123 \pm 0.116$ \\
C852 & $172 \pm-35.522$ & $0.065 \pm 0.049$ & $1.545 \pm 1.167$ & $0.045 \pm 0.029$ \\
C853 & $42.666 \pm-1.7$ & $0.082 \pm 0.003$ & $1.944 \pm 0.101$ & $0.074 \pm 0.053$ \\
C883 & $168.5 \pm-41.041$ & $0.07 \pm 0.042$ & $2.059 \pm 1.494$ & $0.039 \pm 0.018$ \\
C884 & $29.722 \pm-54.486$ & $0.101 \pm 0.048$ & $2.269 \pm 1.09$ & $0.056 \pm 0.03$ \\
C885 & $85.473 \pm-36.948$ & $0.055 \pm 0.028$ & $1.323 \pm 0.696$ & $0.049 \pm 0.021$ \\
C886 & $57.257 \pm-27.06$ & $0.076 \pm 0.027$ & $1.99 \pm 0.795$ & $0.067 \pm 0.044$ \\
C889 & $62.077 \pm-21.949$ & $0.106 \pm 0.041$ & $2.653 \pm 0.967$ & $0.065 \pm 0.032$ \\
C896 & $42.076 \pm-28.397$ & $0.067 \pm 0.043$ & $1.591 \pm 1.036$ & $0.075 \pm 0.078$ \\
All & $47.873 \pm 23.26$ & $0.070 \pm 0.003$ & $1.674 \pm 0.067$ & $0.052 \pm 0.003$ \\
\hline
\end{tabular}

All represent the averages of all stations combined. 


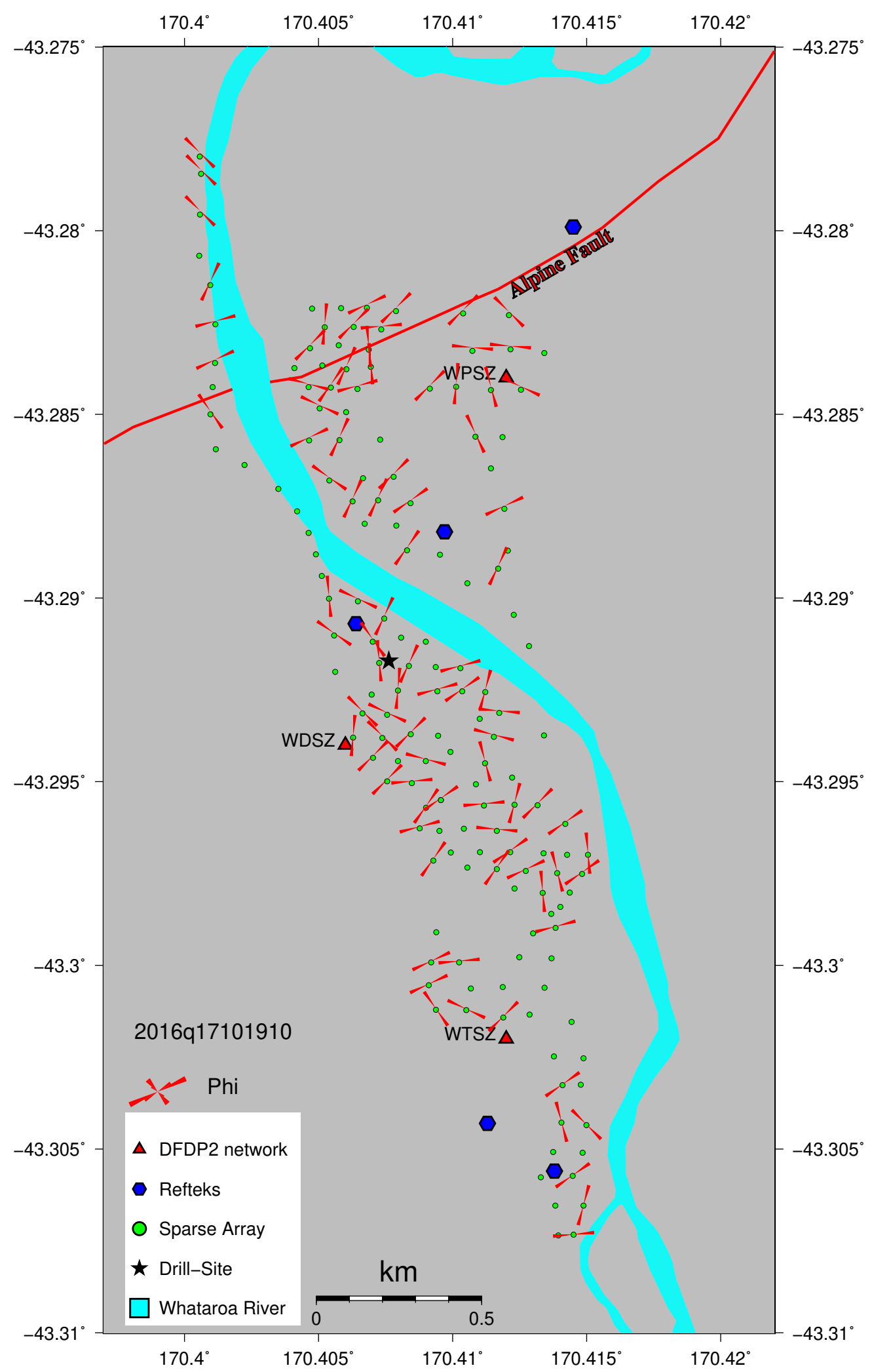

Figure B.1: The spatial distribution of $\phi$ orientation for event 2016q17101910 (AB grade) obtained from local S-phase events is plotted as circular histograms (rose diagrams) the stations at which measurements were made. The red line shows the Alpine fault trace. 


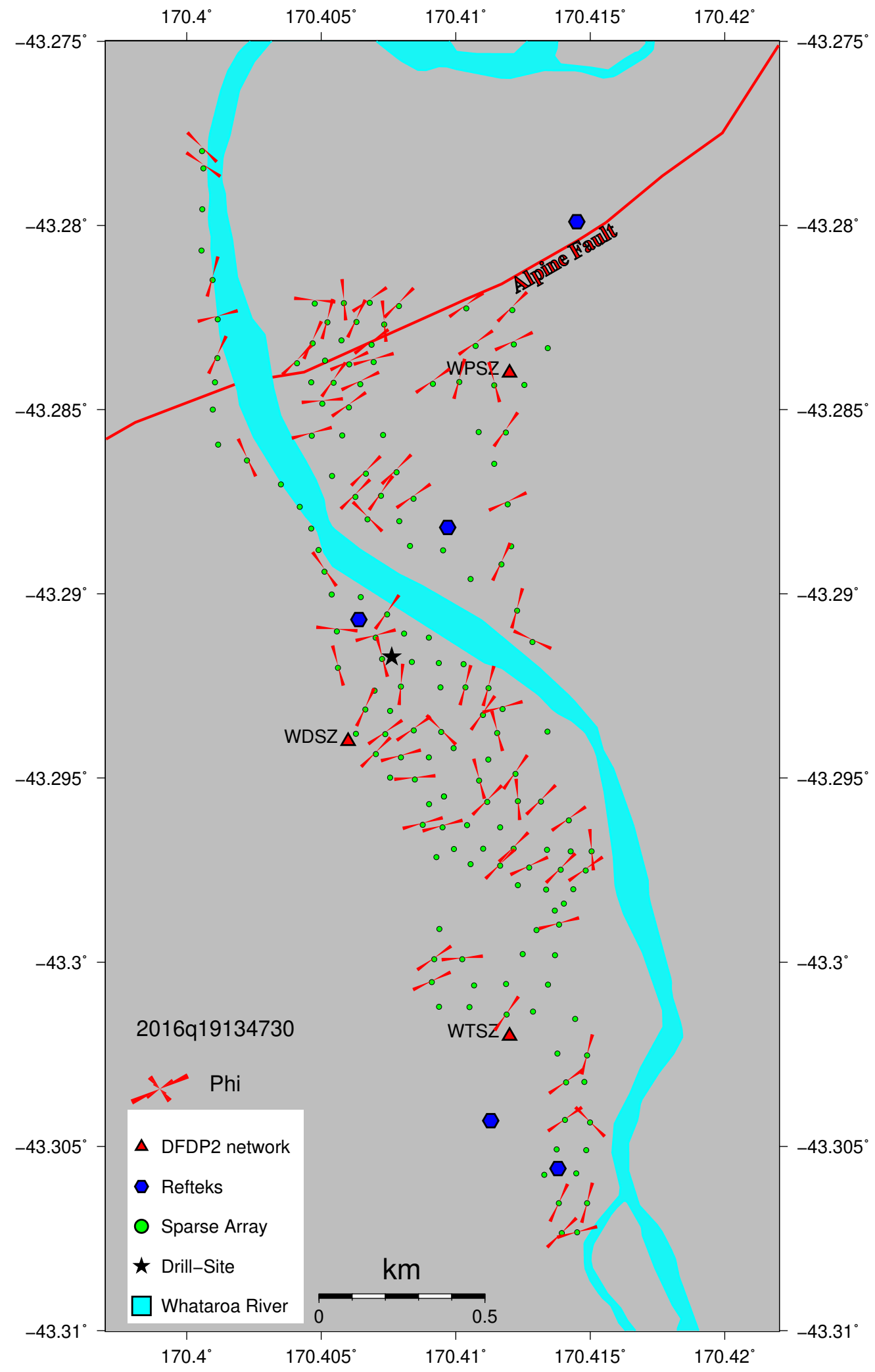

Figure B.2: The spatial distribution of $\phi$ orientation for event 2016q19134730 (AB grade) obtained from local S-phase events is plotted as circular histograms (rose diagrams) the stations at which measurements were made. The red line shows the Alpine fault trace. 


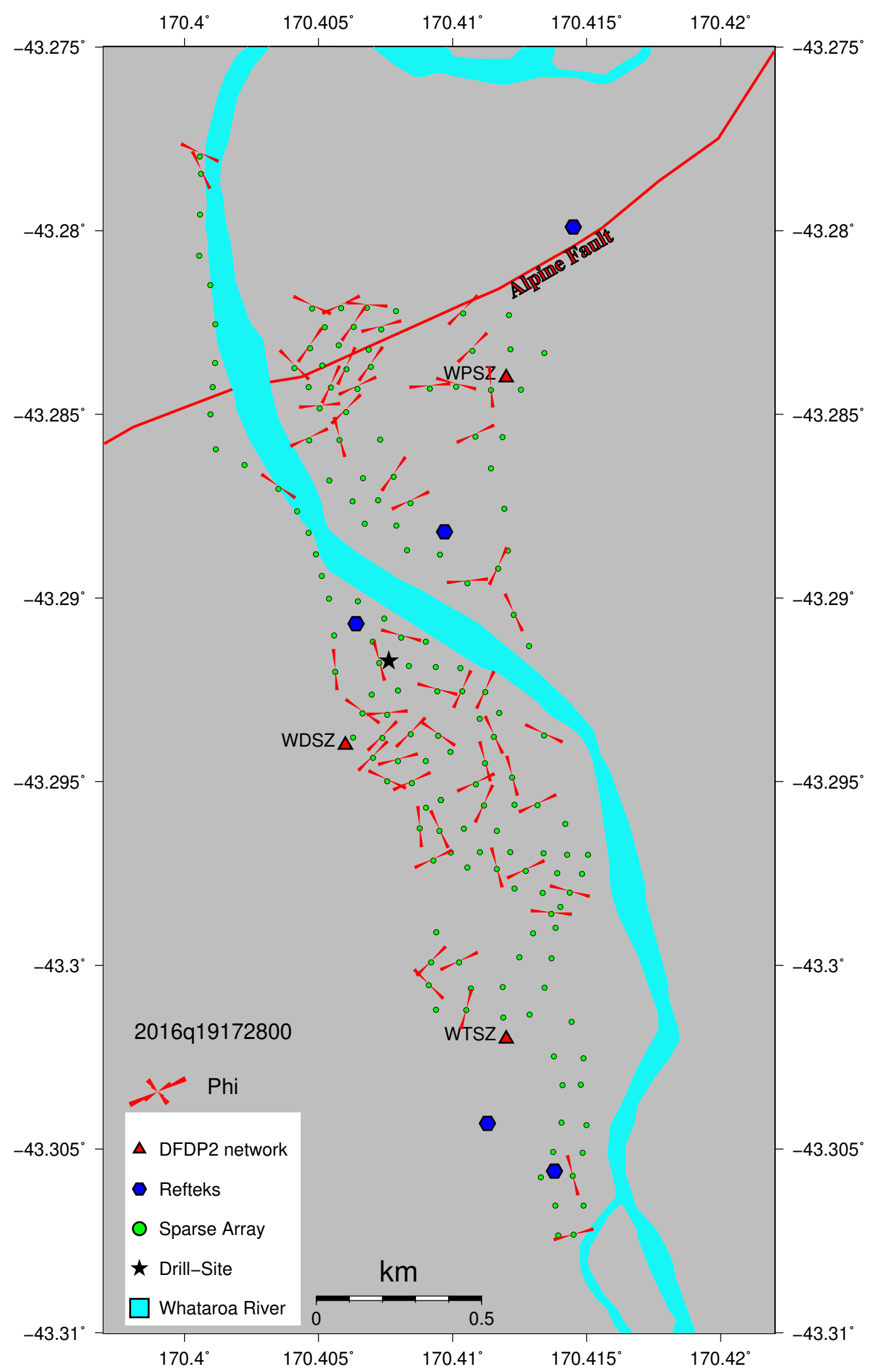

Figure B.3: The spatial distribution of $\phi$ orientation for event 2016q19172800 (AB grade) obtained from local S-phase events is plotted as circular histograms (rose diagrams) the stations at which measurements were made. The red line shows the Alpine fault trace. 


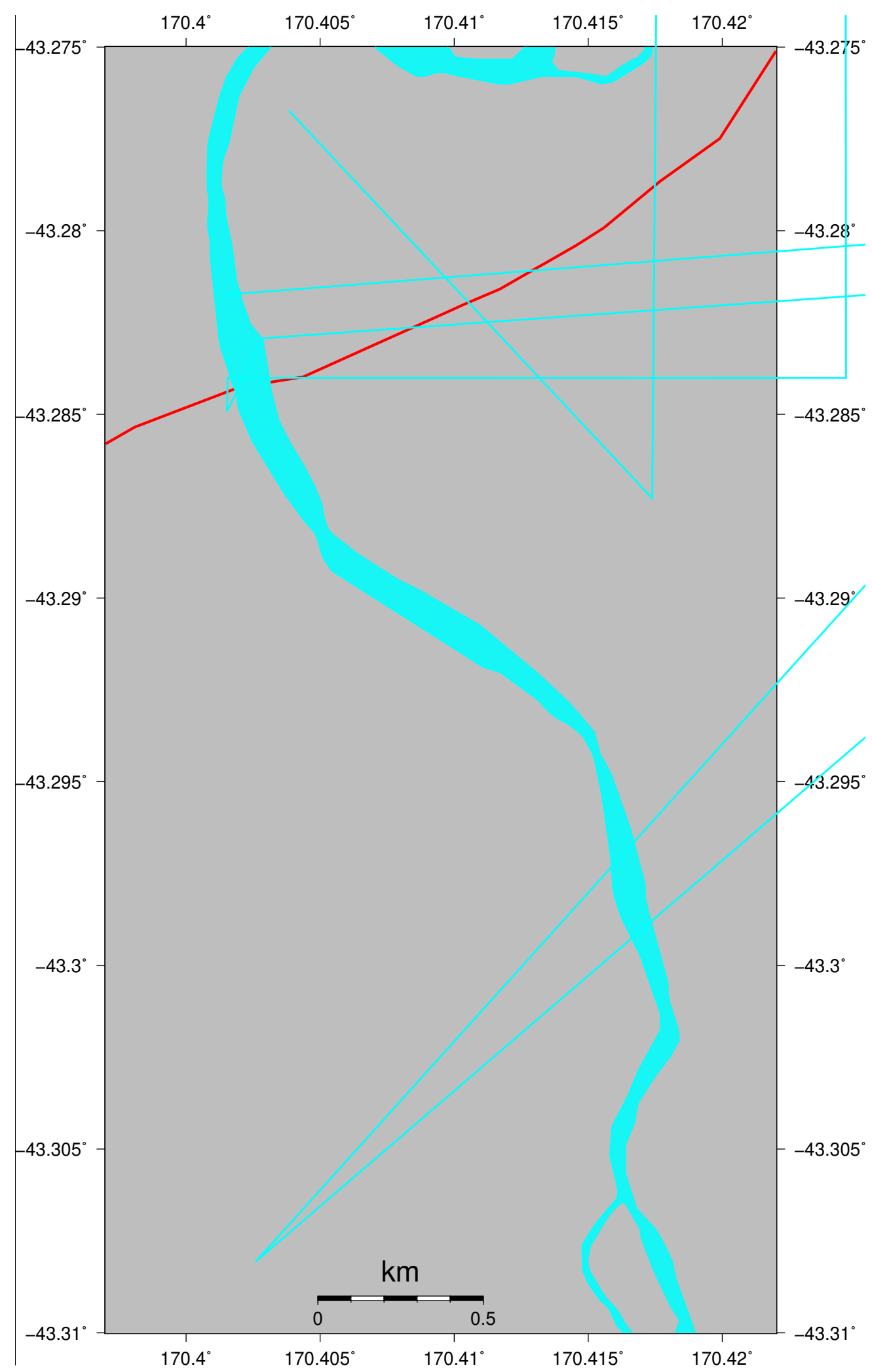

Figure B.4: The spatial distribution of $\phi$ orientation for event 2016q20043517 (AB grade) obtained from local S-phase events is plotted as circular histograms (rose diagrams) the stations at which measurements were made. The red line shows the Alpine fault trace. 


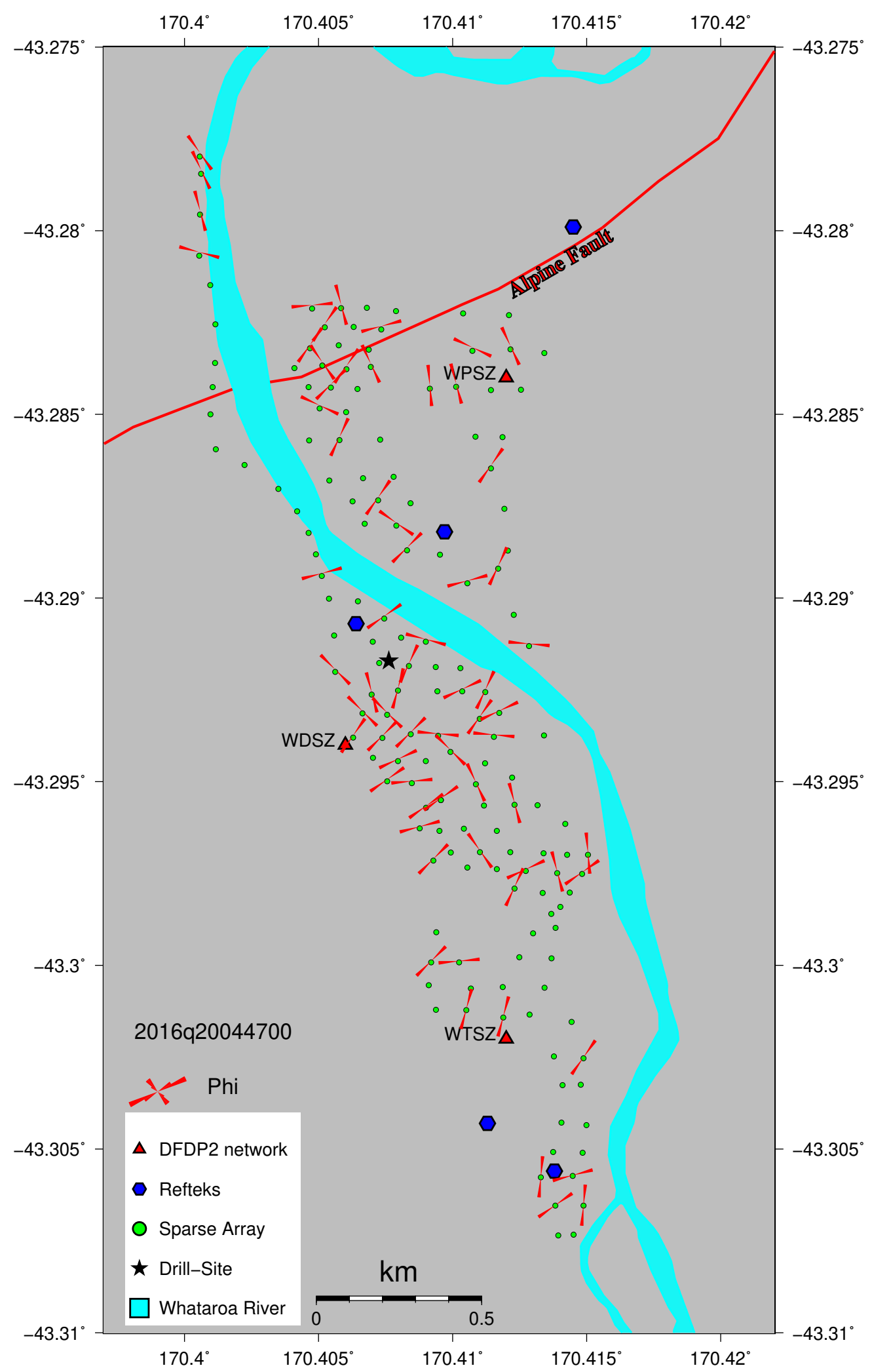

Figure B.5: The spatial distribution of $\phi$ orientation for event 2016q20044700 (AB grade) obtained from local S-phase events is plotted as circular histograms (rose diagrams) the stations at which measurements were made. The red line shows the Alpine fault trace. 


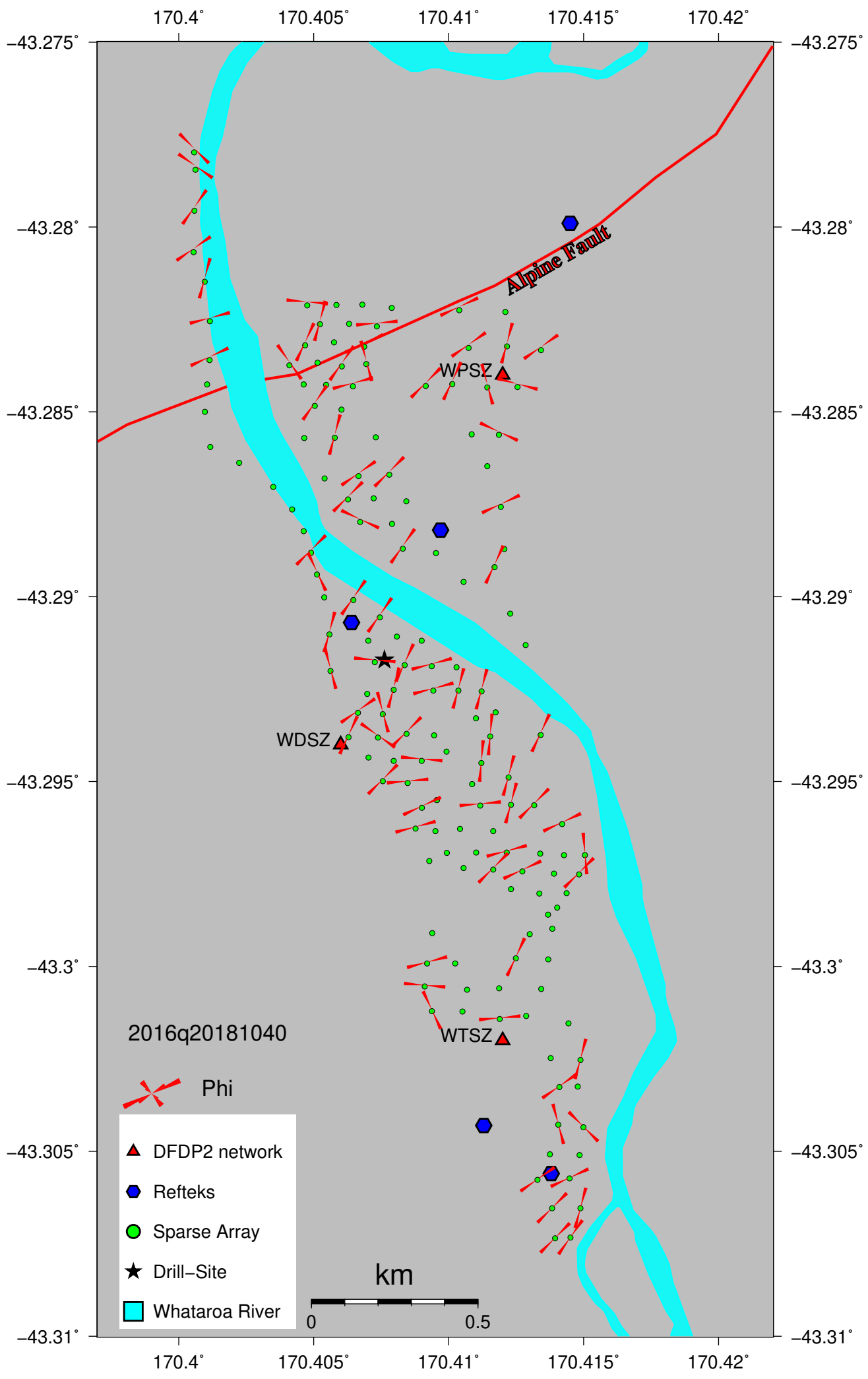

Figure B.6: The spatial distribution of $\phi$ orientation for event (AB grade) obtained from local S-phase events is plotted as circular histograms (rose diagrams) the stations at which measurements were made. The red line shows the Alpine fault trace. 


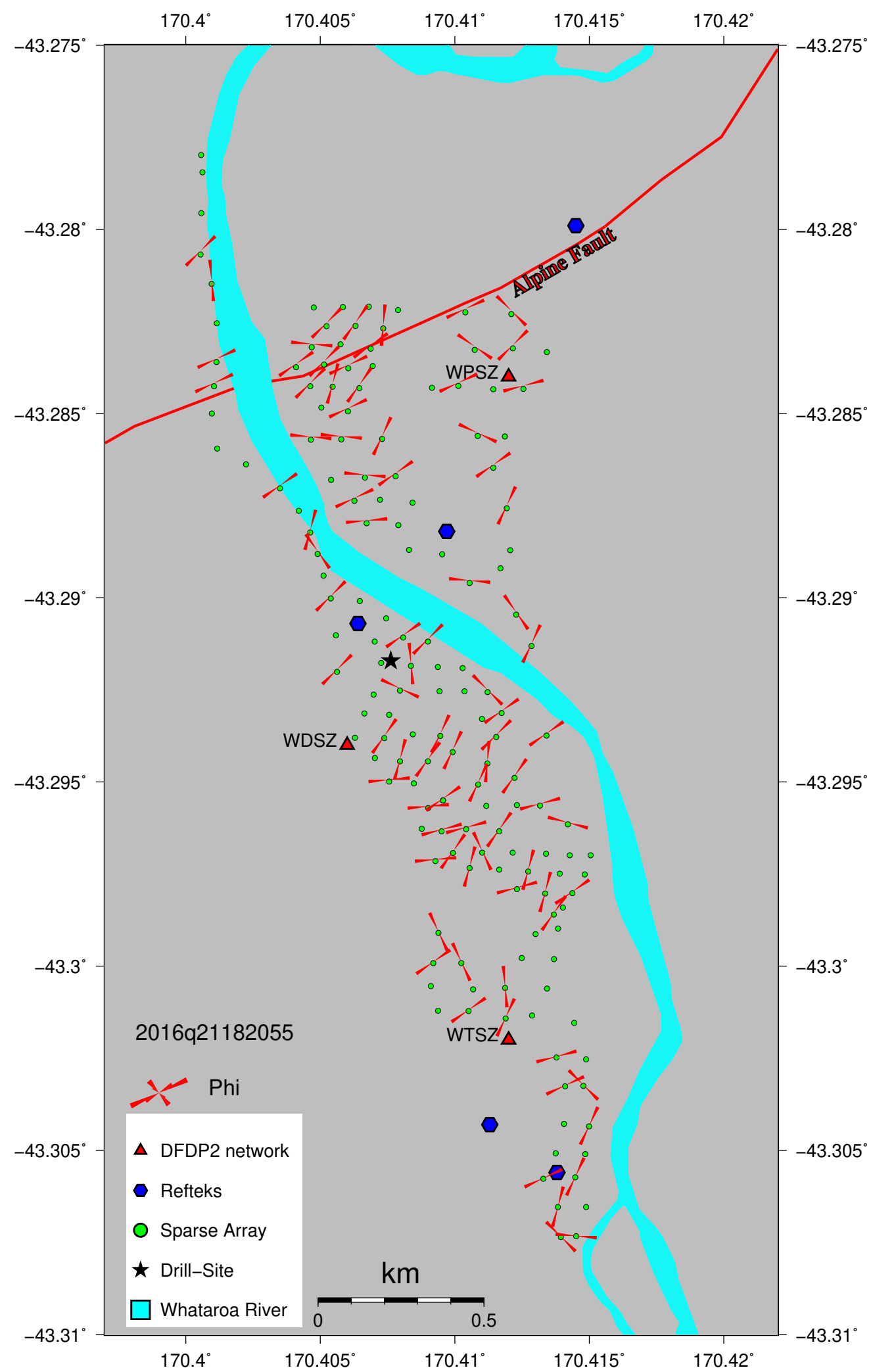

Figure B.7: The spatial distribution of $\phi$ orientation for event 2016q21182055 (AB grade) obtained from local S-phase events is plotted as circular histograms (rose diagrams) the stations at which measurements were made. The red line shows the Alpine fault trace. 


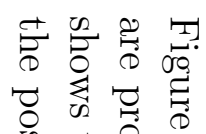

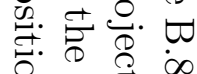

范

串总官

志苍芦莡

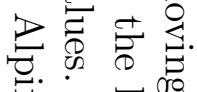

붕

官官泀曷

न के

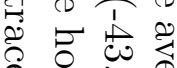

봉

강

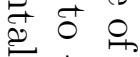

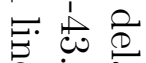

Dن

$8 ?$

官录

๑ क

웅

$+8$

官节节.

if क \&

क्षे

के

○宗导.

की

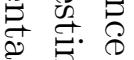

응

윰

$\rightarrow 8$

○

㿣. 泀.

है

क्ष

夷号 8

क्ष

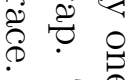

官宫急

ए

हैं

官

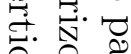

류

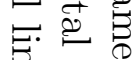

的节

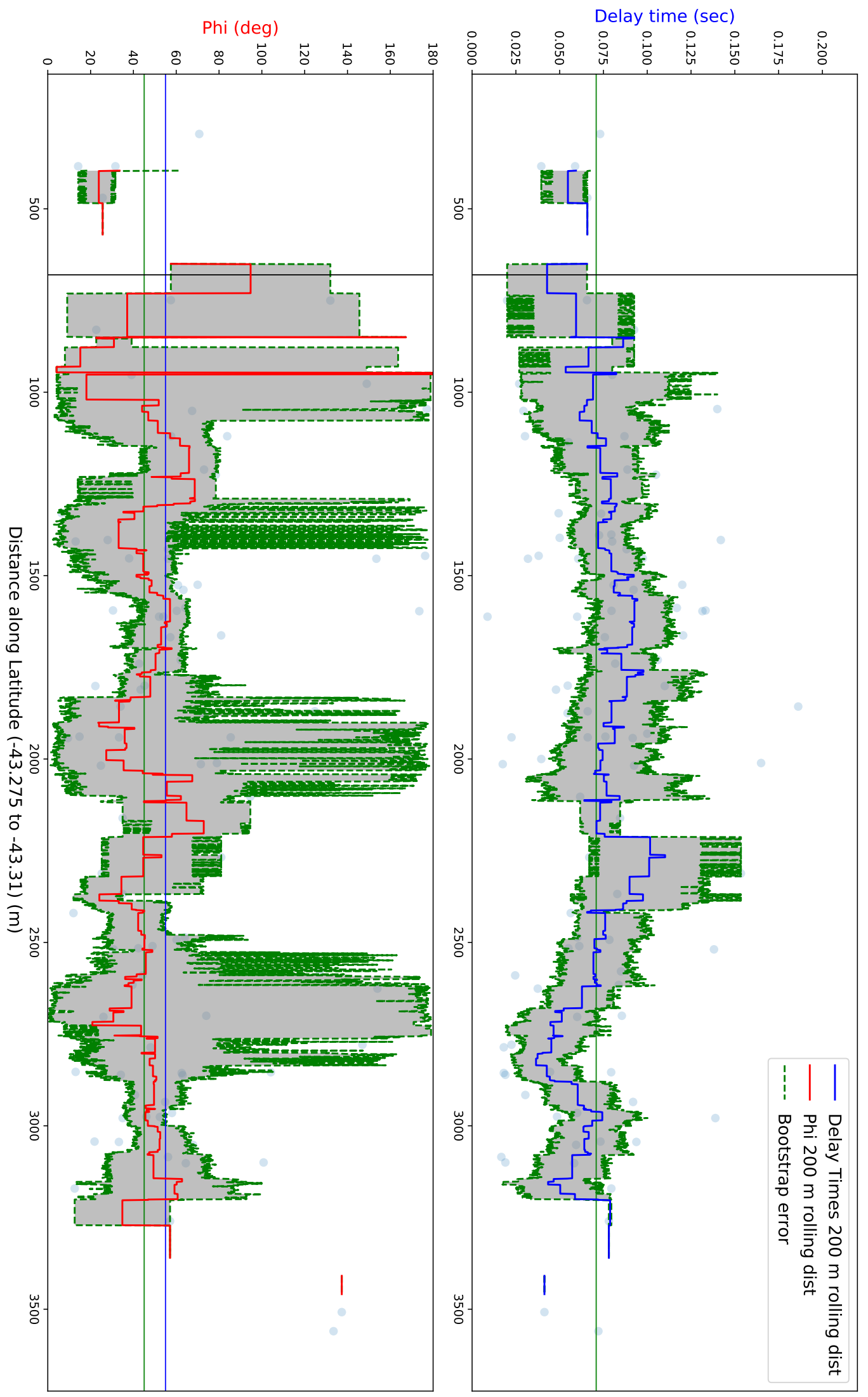




\section{Supporting Information for Chapter 5 "What Causes the Variations in Shear Wave Splitting Measurements"}

\section{Introduction}

This section contains supplemental materials that augment reader's understanding of

Chapter 5. It contains six figures. 

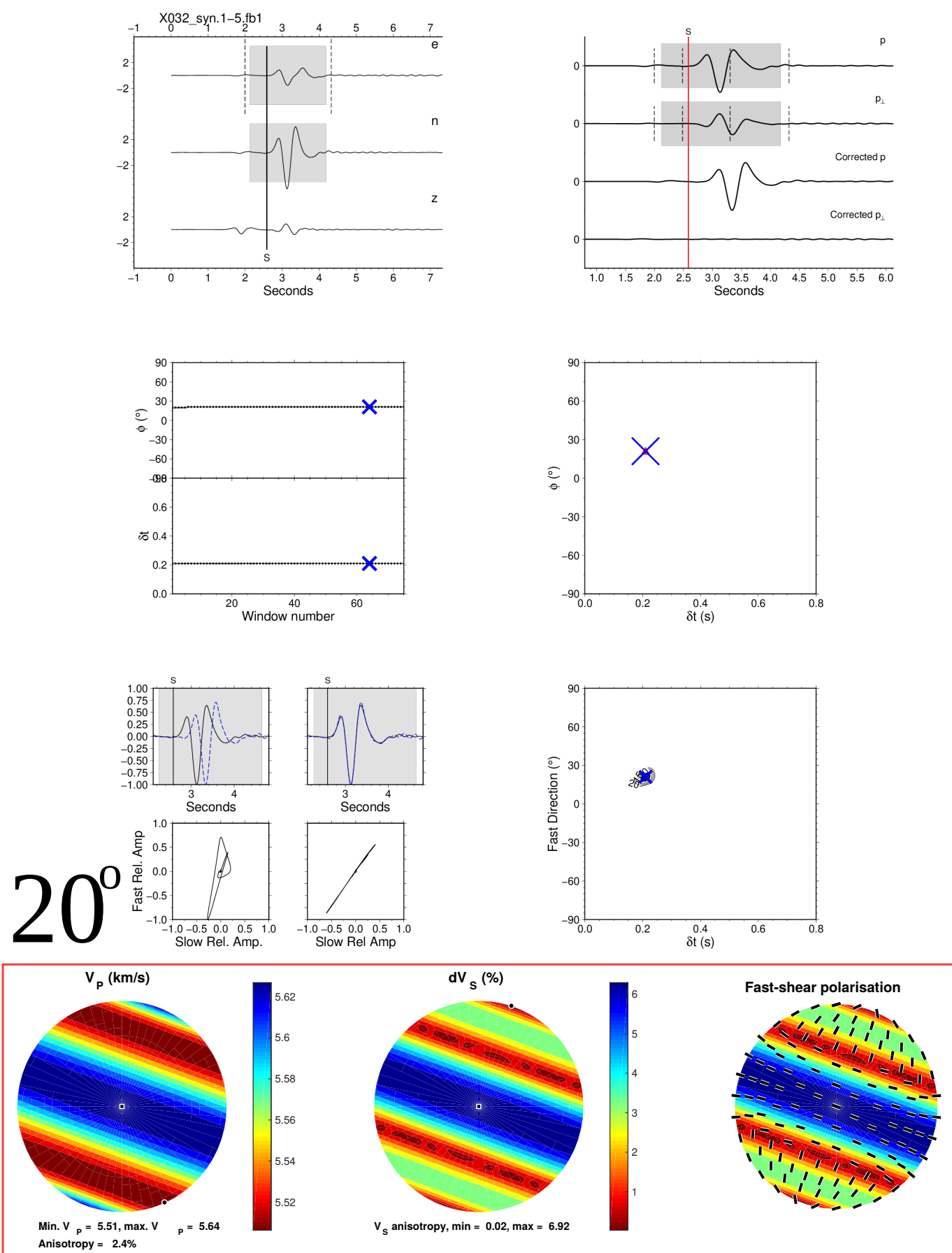

Figure C.1: $20^{\circ}$ rotation of the elasticity matrix/tensor about azimuth. Same description as Figure 5.3. 

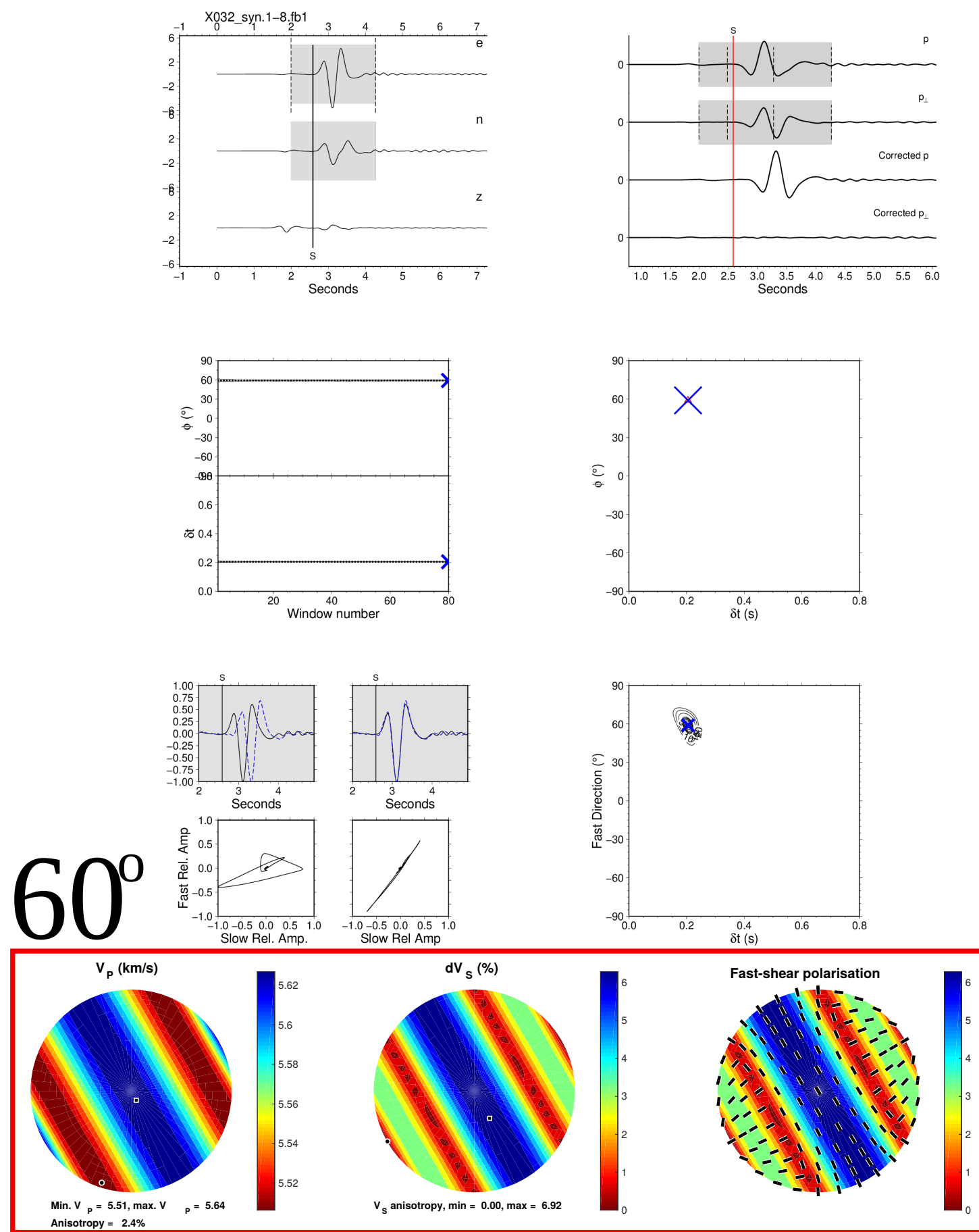

Figure C.2: $60^{\circ}$ rotation of the elasticity matrix/tensor about azimuth. Same description as Figure 5.3. 

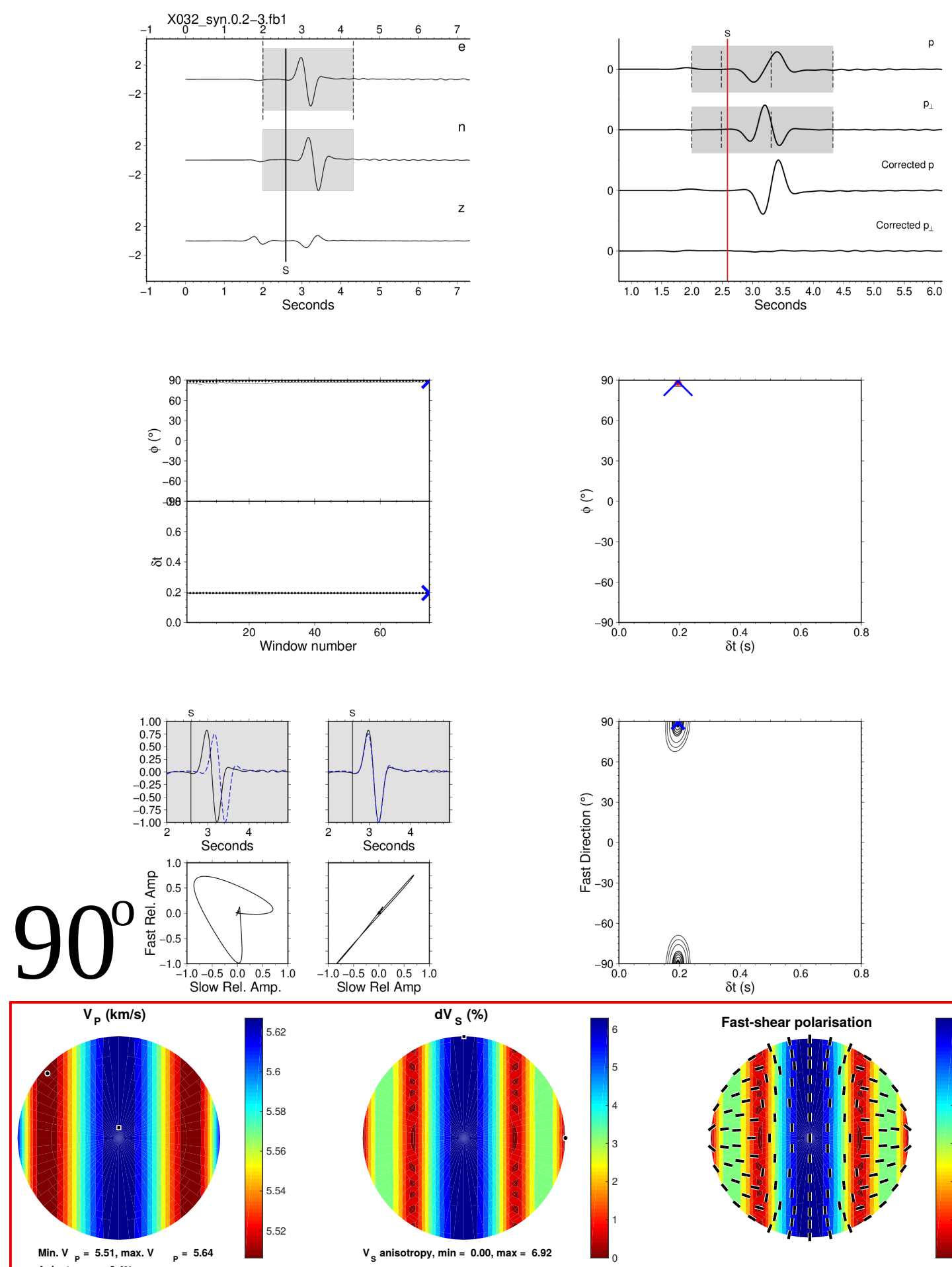

Figure C.3: $90^{\circ}$ rotation of the elasticity matrix/tensor about azimuth. Same description as Figure 5.3. 


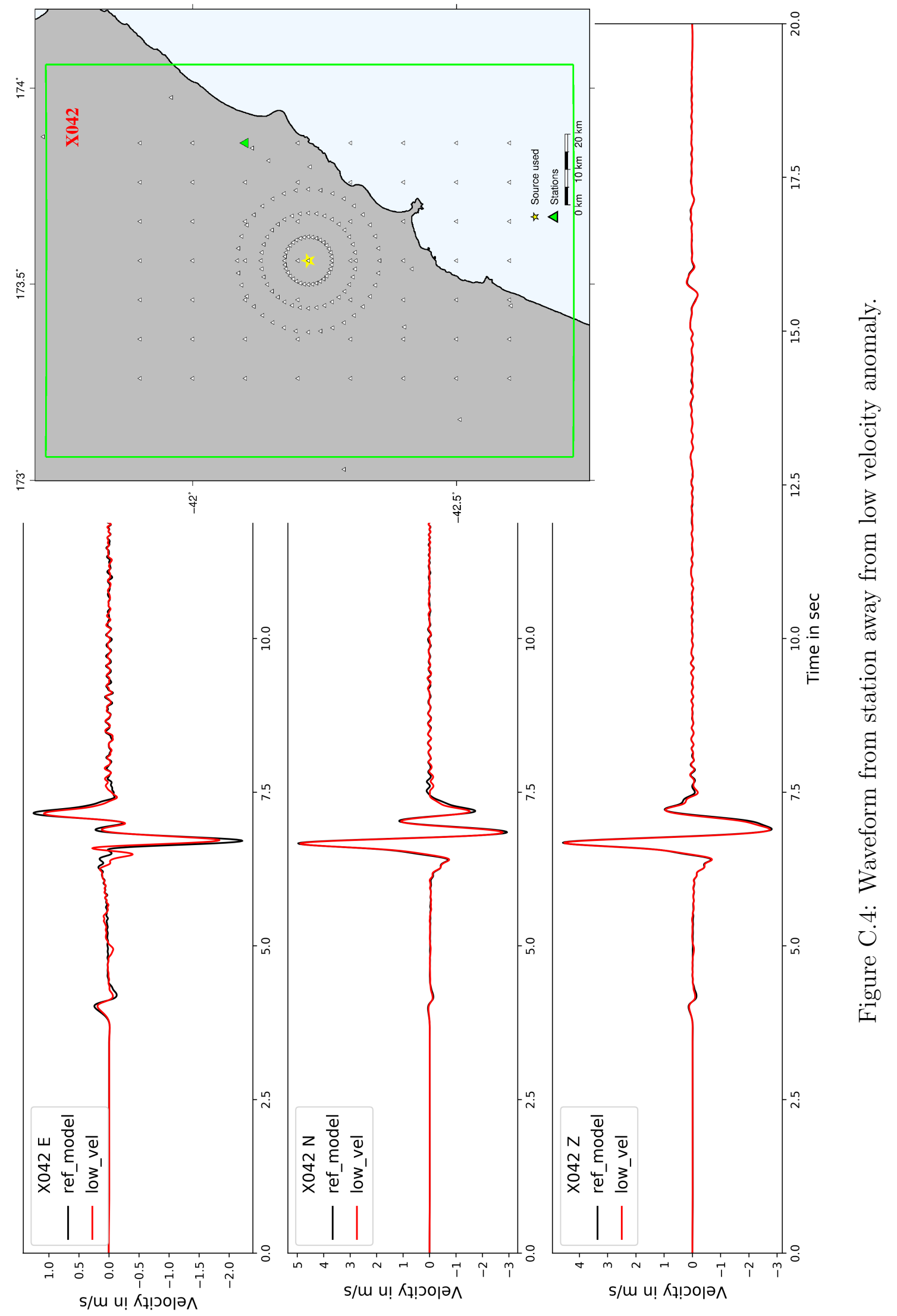




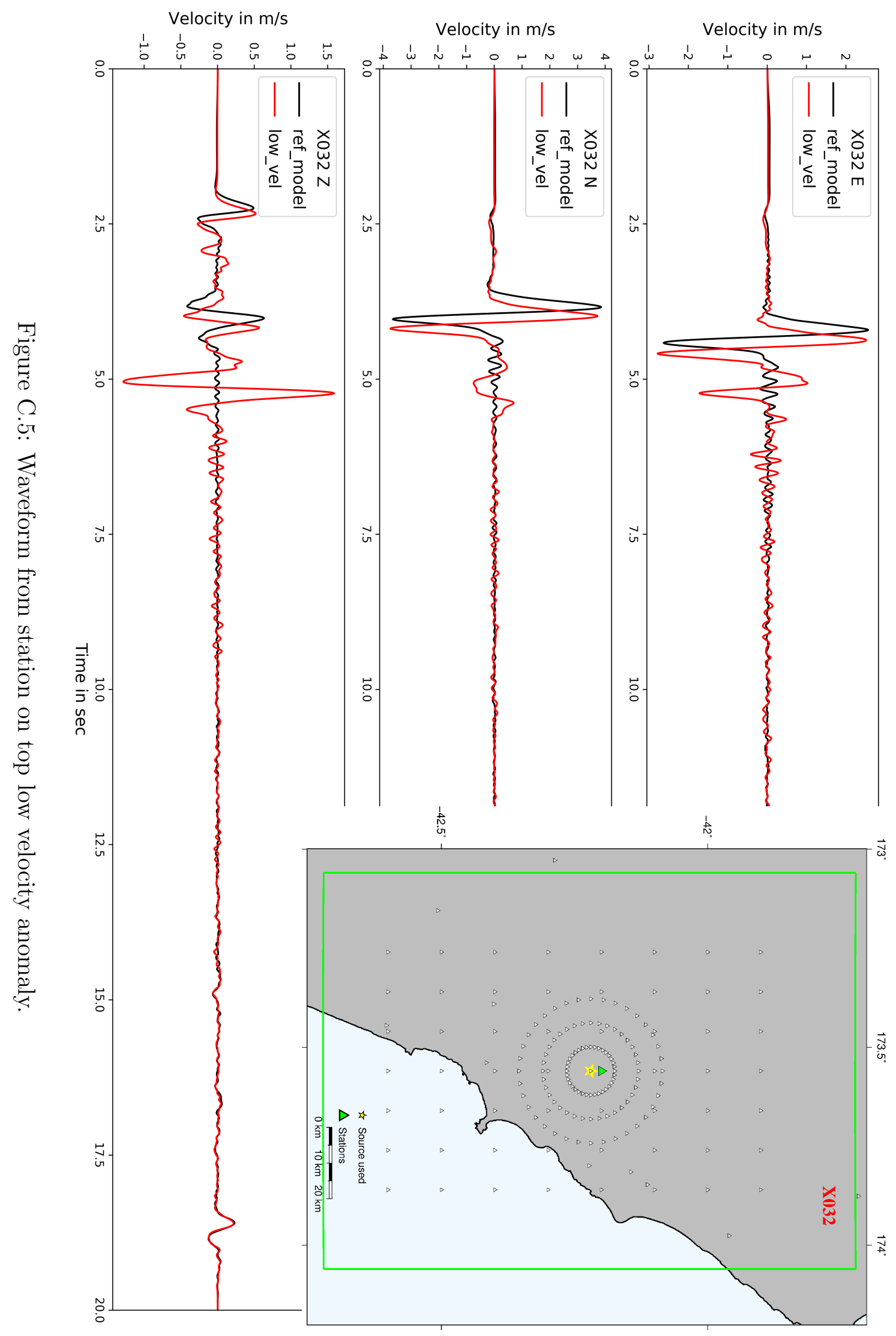




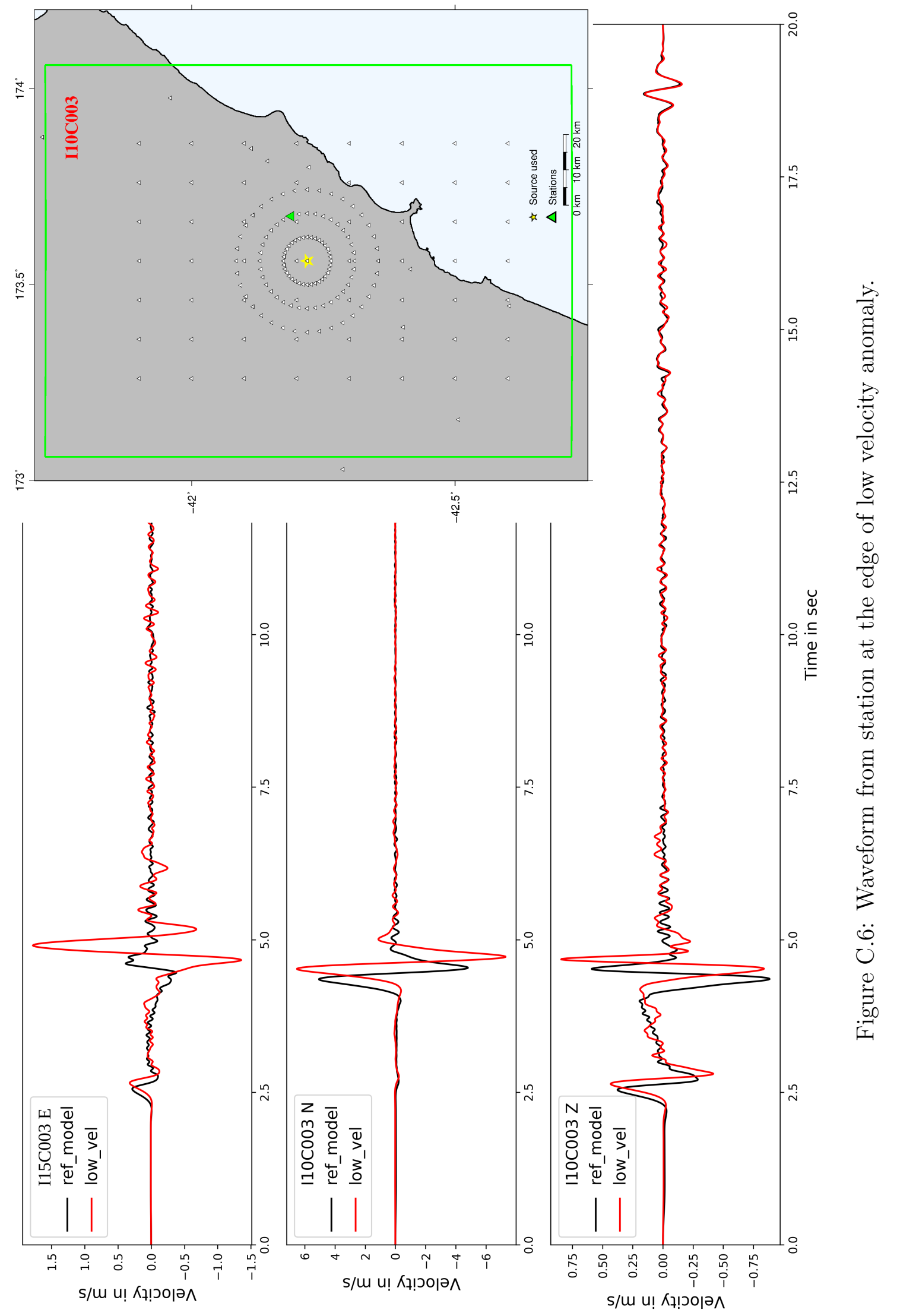




\section{Bibliography}

Aben, F. M., M.-L. Doan, J.-P. Gratier, and F. Renard, Experimental postseismic recovery of fractured rocks assisted by calcite sealing, Geophysical Research Letters, 44(14), 7228-7238, doi:10.1002/2017gl073965, 2017.

Abercrombie, R. E., S. Bannister, J. Ristau, and D. Doser, Variability of earthquake stress drop in a subduction setting, the Hikurangi Margin, New Zealand, Geophysical Journal International, 208(1), 306-320, doi:10.1093/gji/ggw393, 2016.

Adam, L., M. Frehner, K. Sauer, V. Toy, and S. Guerin-Marthe, Seismic Anisotropy and Its Impact on Imaging the Shallow Alpine Fault: An Experimental and Modeling Perspective, Journal of Geophysical Research: Solid Earth, 125(8), doi: 10.1029/2019jb019029, 2020.

Aki, K., and P. G. Richards, Quantitative Seismology, 2nd Ed., University Science Books, 2002.

Allen, R. V., Automatic earthquake recognition and timing from single traces, Bulletin of the Seismological Society of America, 68(5), 1521-1532, 1978.

Anderson, D. L., Theory of the Earth, Blackwell Scientific Publications, 1989.

Anderson, E. M., The dynamics of faulting, Transactions of the Edinburgh Geological Society, 8(3), 387-402, doi:10.1144/transed.8.3.387, 1905.

Anderson, H., T. Webb, and J. Jackson, Focal mechanisms of large earthquakes in the South Island of New Zealand: implications for the accommodation of PacificAustralia plate motion, Geophysical Journal International, 115(3), 1032-1054, doi: 10.1111/j.1365-246x.1993.tb01508.x, 1993. 
Araragi, K. R., M. K. Savage, T. Ohminato, and Y. Aoki, Seismic anisotropy of the upper crust around Mount Fuji, Japan, Journal of Geophysical Research: Solid Earth, 120(4), 2739-2751, doi:10.1002/2014jb011554, 2015.

Arnold, R., and J. Townend, A Bayesian approach to estimating tectonic stress from seismological data, Geophysical Journal International, 170(3), 1336-1356, doi:10. 1111/j.1365-246X.2007.03485.x, 2007.

Aster, R. C., P. M. Shearer, and J. Berger, Quantitative measurements of shear wave polarizations at the anza seismic network, southern california: Implications for shear wave splitting and earthquake prediction, Journal of Geophysical Research, 95(B8), 12,449, doi:10.1029/jb095ib08p12449, 1990.

Aster, R. C., P. M. Shearer, and J. Berger, Reply [to "Comment on 'Quantitative measurements of shear wave polarizations at the Anza Seismic Network, southern California: Implications for shear wave splitting and earthquake prediction' by Richard C. Aster, Peter M. Shearer, and Jon Berger"], Journal of Geophysical Research: Solid Earth, 96 (B4), 6415-6419, doi:10.1029/91jb00294, 1991.

Audoine, E., M. K. Savage, and K. Gledhill, Seismic anisotropy from local earthquakes in the transition region from a subduction to a strike-slip plate boundary, New Zealand, Journal of Geophysical Research: Solid Earth, 105(B4), 8013-8033, doi: 10.1029/1999JB900444, 2000.

Avendonk, H. J. A. V., W. S. Holbrook, D. Okaya, J. K. Austin, F. Davey, and T. Stern, Continental crust under compression: A seismic refraction study of South Island Geophysical Transect I, South Island, New Zealand, Journal of Geophysical Research: Solid Earth, 109(B6), doi:10.1029/2003jb002790, 2004.

Babuska, V., and M. Cara, Seismic anisotropy in the Earth, vol. 10, Springer Science \& Business Media, 1991.

Backus, G. E., Possible forms of seismic anisotropy of the uppermost mantle under oceans, Journal of Geophysical Research, 70(14), 3429-3439, 1965. 
Baisch, S., Scattering change near the earthquake and temporal, Journal of geophysical research, 106(B8), 16-323, 2001.

Bale, R., B. Gratacos, B. Mattocks, S. Roche, K. Poplavskii, and X. Li, Shear wave splitting applications for fracture analysis and improved imaging:some onshore examples, First Break: Data Processing, 2009.

Balfour, N., M. Savage, and J. Townend, Stress and crustal anisotropy in Marlborough, New Zealand: evidence for low fault strength and structure-controlled anisotropy, Geophysical Journal International, 163(3), 1073-1086, 2005.

Balfour, N., J. F. Cassidy, and S. E. Dosso, Crustal anisotropy in the forearc of the Northern Cascadia Subduction Zone, British Columbia, Geophysical Journal International, 188(1), 165-176, doi:10.1111/j.1365-246X.2011.05231.x, 2012.

Bao, H., J. Bielak, O. Ghattas, L. F. Kallivokas, D. R. O'Hallaron, J. R. Shewchuk, and J. Xu, Large-scale simulation of elastic wave propagation in heterogeneous media on parallel computers, Computer Methods in Applied Mechanics and Engineering, 152(1-2), 85-102, doi:10.1016/s0045-7825(97)00183-7, 1998.

Baratin, L.-M., C. J. Chamberlain, J. Townend, and M. K. Savage, Focal mechanisms and inter-event times of low-frequency earthquakes reveal quasi-continuous deformation and triggered slow slip on the deep Alpine Fault, Earth and Planetary Science Letters, 484, 111-123, doi:10.1016/j.epsl.2017.12.021, 2018.

Barruol, G., and D. Mainprice, A quantitative evaluation of the contribution of crustal rocks to the shear-wave splitting of teleseismic SKS waves, Physics of the Earth and Planetary Interiors, 78(3-4), 281-300, doi:10.1016/0031-9201(93)90161-2, 1993.

Barth, N. C., V. G. Toy, R. M. Langridge, and R. J. Norris, Scale dependence of oblique plate-boundary partitioning: New insights from LiDAR, central Alpine fault, New Zealand, Lithosphere, 4(5), 435-448, doi:10.1130/1201.1, 2012.

Beavan, J., M. Moore, C. Pearson, M. Henderson, B. Parsons, S. Bourne, P. England, D. Walcott, G. Blick, D. Darby, and K. Hodgkinson, Crustal deformation during 
1994-1998 due to oblique continental collision in the central Southern Alps, New Zealand, and implications for seismic potential of the Alpine fault, Journal of Geophysical Research: Solid Earth, 104(B11), 25,233-25,255, doi:10.1029/1999jb900198, 1999.

Beavan, J., P. Denys, M. Denham, B. Hager, T. Herring, and P. Molnar, Distribution of present-day vertical deformation across the Southern Alps, New Zealand, from 10 years of GPS data, Geophysical Research Letters, 37 (16), doi:10.1029/2010gl044165, 2010.

Beavan, J., L. M. Wallace, N. Palmer, P. Denys, S. Ellis, N. Fournier, S. Hreinsdottir, C. Pearson, and M. Denham, New Zealand GPS velocity field: 1995-2013, New Zealand Journal of Geology and Geophysics, 59(1), 5-14, doi:10.1080/00288306.2015. 1112817, 2016.

Berens, P., CircStat: AMATLABToolbox for circular statistics, Journal of Statistical Software, 31(10), doi:10.18637/jss.v031.i10, 2009.

Bernth, H., and C. Chapman, A comparison of the dispersion relations for anisotropic elastodynamic finite-difference grids, Geophysics, 76(3), WA43-WA50, doi:10.1190/ $1.3555530,2011$.

Bianco, F., L. Scarfı, E. D. Pezzo, and D. Patanè, Shear wave splitting changes associated with the 2001 volcanic eruption on Mt Etna, Geophysical Journal International, 167(2), 959-967, doi:10.1111/j.1365-246x.2006.03152.x, 2006.

Boese, C., Microseismicity in the central Southern Alps, Westland, New Zealand, Ph.D. thesis, Victoria University of Wellington, 2012.

Boness, N., and M. Zoback, Mapping stress and structurally controlled crustal shear velocity anisotropy in California, Geology, 34(10), 825-828, 2006.

Boore, D. M., A note on the effect of simple topography on seismic SH waves, Bulletin of the seismological society of America, 62(1), 275-284, 1972. 
Booth, D. C., and S. Crampin, Shear-wave polarizations on a curved wavefront at an isotropic free surface, Geophysical Journal International, 83(1), 31-45, doi:10.1111/ j.1365-246x.1985.tb05154.x, 1985.

Boulton, C., D. E. Moore, D. A. Lockner, V. G. Toy, J. Townend, and R. Sutherland, Frictional properties of exhumed fault gouges in DFDP-1 cores, Alpine Fault, New Zealand, Geophysical Research Letters, 41(2), 356-362, doi:10.1002/2013gl058236, 2014.

Bourguignon, S., S. Bannister, C. M. Henderson, J. Townend, and H. Zhang, Structural heterogeneity of the midcrust adjacent to the central Alpine Fault, New Zealand: Inferences from seismic tomography and seismicity between Harihari and Ross, Geochemistry, Geophysics, Geosystems, 16(4), 1017-1043, doi:10.1002/2014gc005702, 2015.

Canuto, C., M. Y. Hussaini, A. Quarteroni, and T. A. Zang, Spectral Methods in Fluid Dynamics, Springer Berlin Heidelberg, doi:10.1007/978-3-642-84108-8, 1988.

Casarotti, E., M. Stupazzini, S. J. Lee, D. Komatitsch, A. Piersanti, and J. Tromp, GEOCUBIT, an HPC parallel mesher for spectral-element method seismic wave simulation, in 70th EAGE Conference and Exhibition - Workshops and Fieldtrips, European Association of Geoscientists \& Engineers, doi:10.3997/2214-4609.201405029, 2008b.

Castellazzi, C., M. Savage, E. Walsh, and R. Arnold, Shear wave automatic picking and splitting measurements at Ruapehu volcano, New Zealand, Journal of Geophysical Research: Solid Earth, 120, 3363-3384, 2015.

Cesca, S., Y. Zhang, V. Mouslopoulou, R. Wang, J. Saul, M. Savage, S. Heimann, S.-K. Kufner, O. Oncken, and T. Dahm, Complex rupture process of the Mw 7.8, 2016, Kaikōura earthquake, New Zealand, and its aftershock sequence, Earth and Planetary Science Letters, 478, 110-120, doi:10.1016/j.epsl.2017.08.024, 2017.

Chadwick, P., M. Vianello, and S. C. Cowin, A new proof that the number of linear 
elastic symmetries is eight, Journal of the Mechanics and Physics of Solids, 49(11), 2471-2492, doi:10.1016/s0022-5096(01)00064-3, 2001.

Chaljub, E., D. Komatitsch, J.-P. Vilotte, Y. Capdeville, B. Valette, and G. Festa, Spectral-element analysis in seismology, in Advances in Wave Propagation in Heterogenous Earth, pp. 365-419, Elsevier, doi:10.1016/s0065-2687(06)48007-9, 2007.

Chamberlain, C. J., D. R. Shelly, J. Townend, and T. A. Stern, Low-frequency earthquakes reveal punctuated slow slip on the deep extent of the Alpine Fault, New Zealand, Geochemistry, Geophysics, Geosystems, 15(7), 2984-2999, doi:10.1002/ 2014gc005436, 2014

Chamberlain, C. J., C. M. Boese, and J. Townend, Cross-correlation-based detection and characterisation of microseismicity adjacent to the locked, late-interseismic Alpine Fault, South Westland, New Zealand, Earth and Planetary Science Letters, 457, 63-72, doi:10.1016/j.epsl.2016.09.061, 2017a.

Chamberlain, C. J., C. M. Boese, J. D. Eccles, M. K. Savage, L.-M. Baratin, J. Townend, and et al., Real-Time Earthquake Monitoring during the Second Phase of the Deep Fault Drilling Project, Alpine Fault, New Zealand, Seismological Research Letters, 88(6), 1443-1454, doi:10.1785/0220170095, 2017b.

Chen, M., and J. Tromp, Theoretical and numerical investigations of global and regional seismic wave propagation in weakly anisotropic earth models, Geophysical Journal International, 168(3), 1130-1152, doi:10.1111/j.1365-246x.2006.03218.x, 2007.

Chen, X., A systematic and efficient method of computing normal modes for multilayered half-space, Geophysical Journal International, 115(2), 391-409, doi:10.1111/j. 1365-246x.1993.tb01194.x, 1993.

Chen, Y., Z. Zhang, C. Sun, and J. Badal, Crustal anisotropy from Moho converted Ps wave splitting analysis and geodynamic implications beneath the eastern margin of 
Tibet and surrounding regions, Gondwana Research, 24(3-4), 946-957, doi:10.1016/ j.gr.2012.04.003, 2013.

Chevrot, S., Multichannel analysis of shear wave splitting, Journal of Geophysical Research: Solid Earth, 105(B9), 21,579-21,590, doi:10.1029/2000jb900199, 2000.

Chevrot, S., N. Favier, and D. Komatitsch, Shear wave splitting in three-dimensional anisotropic media, Geophysical Journal International, 159(2), 711-720, doi:10.1111/ j.1365-246X.2004.02432.x, 2004.

Chiarabba, C., P. D. Gori, and E. Boschi, Pore-pressure migration along a normalfault system resolved by time-repeated seismic tomography, Geology, 37(1), 67-70, doi:10.1130/g25220a.1, 2009.

Christensen, N. I., Poisson's ratio and crustal seismology, Journal of Geophysical Research: Solid Earth, 101(B2), 3139-3156, doi:10.1029/95jb03446, 1996.

Ciucu, C., Maximum Stress at a Tectonic Fault Plane. Coulomb Law, Romanian Reports in Physics, 62(2), 414-421, 2010.

Clark, K., E. Nissen, J. Howarth, I. Hamling, J. Mountjoy, W. Ries, K. Jones, S. Goldstien, U. Cochran, P. Villamor, S. Hreinsdóttir, N. Litchfield, C. Mueller, K. Berryman, and D. Strong, Highly variable coastal deformation in the $2016 \mathrm{Mw}$ 7.8 Kaikōura earthquake reflects rupture complexity along a transpressional plate boundary, Earth and Planetary Science Letters, 474, 334-344, doi:10.1016/j.epsl. 2017.06.048, 2017.

Cochran, E., and K. Kroll, Stress- and structure-controlled anisotropy in a region of complex faulting-Yuha Desert, California, Geophysical Journal International, 202(2), 1109-1121, doi:10.1093/gji/ggv191, 2015.

Cochran, E. S., Y.-G. Li, and J. E. Vidale, Anisotropy in the shallow crust observed around the San Andreas fault before and after the 2004 M 6.0 Parkfield earthquake, Bulletin of the Seismological Society of America, 96(4B), S364-S375, 2006. 
Cox, S. C., G. S. (NZ), and D. J. A. Barrell, Geology of the Aoraki Area: Scale 1: 250000, GNS Science, 2008.

Crampin, S., A review of wave motion in anisotropic and cracked elastic-media, Wave Motion, 3(4), 343-391, doi:10.1016/0165-2125(81)90026-3, 1981.

Crampin, S., An introduction to wave propagation in anisotropic media, Geophysical Journal International, 76(1), 17-28, doi:10.1111/j.1365-246X.1984.tb05018.x, 1984.

Crampin, S., Evaluation of anisotropy by shear-wave splitting, Geophysics, 50(1), 142 152, doi:10.1190/1.1441824, 1985.

Crampin, S., Extensive-dilatancy anisotropy, Eos, Transactions American Geophysical Union, 69(29), 729-729, doi:10.1029/88EO01023, 1988.

Crampin, S., The scattering of shear-waves in the crust, Pure and Applied Geophysics PAGEOPH, 132(1-2), 67-91, doi:10.1007/bf00874358, 1990a.

Crampin, S., The potential of shear-wave VSPs for monitoring recovery: A letter to management, The Leading Edge, 9(3), 50-52, doi:10.1190/1.1439728, 1990b.

Crampin, S., The fracture criticality of crustal rocks, Geophysical Journal International, 118(2), 428-438, doi:10.1111/j.1365-246x.1994.tb03974.x, 1994.

Crampin, S., and D. C. Booth, Shear-wave splitting showing hydraulic dilation of preexisting joints in granite, Scientific Drilling, 1(1), 21-26, 1989.

Crampin, S., and Y. Gao, A review of techniques for measuring shear-wave splitting above small earthquakes, Physics of the Earth and Planetary Interiors, 159(1-2), 1-14, doi:10.1016/j.pepi.2006.06.002, 2006.

Crampin, S., and Y. Gao, The New Geophysics, Terra Nova, 25(3), 173-180, doi: 10.1111/ter.12030, 2013.

Crampin, S., and S. Peacock, A review of the current understanding of seismic shearwave splitting in the Earth's crust and common fallacies in interpretation, Wave Motion, 45(6), 675-722, doi:10.1016/j.wavemoti.2008.01.003, 2008. 
Crampin, S., and S. V. Zatsepin, Modelling the compliance of crustal rock-II. Response to temporal changes before earthquakes, Geophysical Journal International, 129(3), 495-506, doi:10.1111/j.1365-246x.1997.tb04489.x, 1997.

Crampin, S., T. Volti, and R. Stefánsson, A successfully stress-forecast earthquake, Geophysical Journal International, 138(1), F1-F5, doi:10.1046/j.1365-246x.1999. 00891.x, 1999.

Crampin, S., Y. Gao, and J. Bukits, A review of retrospective stress-forecasts of earthquakes and eruptions, Physics of the Earth and Planetary Interiors, 245, 76-87, doi:10.1016/j.pepi.2015.05.008, 2015.

Crotwell, H. P., T. J. Owens, and J. Ritsema, The TauP Toolkit: Flexible Seismic Travel-time and Ray-path Utilities, Seismological Research Letters, 70(2), 154-160, doi:10.1785/gssrl.70.2.154, 1999 .

Currie, C. A., J. F. Cassidy, R. D. Hyndman, and M. G. Bostock, Shear wave anisotropy beneath the Cascadia subduction zone and western North American craton, Geophysical Journal International, 157(1), 341-353, doi:10.1111/j.1365-246x.2004.02175.x, 2004.

Dainty, A. M., The Influence of Seismic Scattering on Monitoring, in Monitoring a Comprehensive Test Ban Treaty, pp. 663-688, Springer Nature, doi:10.1007/978-94011-0419-7_36, 1996.

Davey, F., T. Henyey, W. Holbrook, D. Okaya, T. Stern, A. Melhuish, S. Henrys, H. Anderson, D. Eberhart-Phillips, T. McEvilly, R. Uhrhammer, F. Wu, G. Jiracek, P. Wannamaker, G. Caldwell, and N. Christensen, Preliminary results from a geophysical study across a modern, continent-continent collisional plate boundary — the Southern Alps, New Zealand, Tectonophysics, 288(1-4), 221-235, doi: 10.1016/s0040-1951(97)00297-7, 1998.

Dellow, S., C. Massey, S. Cox, G. Archibald, J. Begg, Z. Bruce, and et al., Landslides caused by the 14 November 2016 Mw7.8 Kaikōura earthquake and the immediate 
response, Bulletin of the New Zealand Society for Earthquake Engineering, 50(2), 106-116, 2017.

Dempsey, E. D., D. J. Prior, E. Mariani, V. G. Toy, and D. J. Tatham, Mica-controlled anisotropy within mid-to-upper crustal mylonites: an EBSD study of mica fabrics in the Alpine Fault Zone, New Zealand, Geological Society, London, Special Publications, 360(1), 33-47, doi:10.1144/sp360.3, 2011.

Diehl, T., N. Deichmann, E. Kissling, and S. Husen, Automatic S-Wave Picker for Local Earthquake Tomography, Bulletin of the Seismological Society of America, $99(3)$, 1906-1920, doi:10.1785/0120080019, 2009.

Dieterich, J. H., Time-dependent friction in rocks, Journal of Geophysical Research, r7(20), 3690-3697, doi:10.1029/jb077i020p03690, 1972.

Dodge, D. A., and G. C. Beroza, Source array analysis of coda waves near the 1989 Loma Prieta, California, mainshock: Implications for the mechanism of coseismic velocity changes, Journal of Geophysical Research: Solid Earth, 102(B11), 24,43724,458, doi:10.1029/97jb02024, 1997.

Duputel, Z., and L. Rivera, Long-period analysis of the 2016 Kaikōura earthquake, Physics of the Earth and Planetary Interiors, 265, 62-66, doi:10.1016/j.pepi.2017. $02.004,2017$.

Durufle, M., P. Grob, and P. Joly, Influence of gauss and gauss-lobatto quadrature rules on the accuracy of a quadrilateral finite element method in the time domain, Numerical Methods for Partial Differential Equations, 25(3), 526-551, doi:10.1002/ num.20353, 2009.

Dvorkin, J., G. Mavko, and A. Nur, Overpressure detection from compressional- and shear-wave data, Geophysical Research Letters, 26(22), 3417-3420, doi:10.1029/ 1999gl008382, 1999.

Eberhart-Phillips, D., and S. Bannister, 3-D imaging of Marlborough, New Zealand, 
subducted plate and strike-slip fault systems, Geophysical Journal International, doi:10.1111/j.1365-246x.2010.04621.x, 2010.

Eberhart-Phillips, D., and B. Fry, Joint local earthquake and teleseismic inversion for 3-D velocity and Q in New Zealand, Physics of the Earth and Planetary Interiors, 283, 48-66, doi:10.1016/j.pepi.2018.08.005, 2018.

Evans, R., Effects of the free surface on shear wavetrains, Geophysical Journal of the Royal Astronomical Society, 76(1), 165-172, doi:\{10.1111/j.1365-246X.1984. tb05032.x\}, 1984.

Evanzia, D., T. Wilson, M. K. Savage, S. Lamb, and H. Hirschberg, Stress Orientations in a Locked Subduction Zone at the Southern Hikurangi Margin, New Zealand, Journal of Geophysical Research: Solid Earth, 122(10), 7895-7911, doi:10.1002/ 2017jb013998, 2017.

Faccioli, E., F. Maggio, R. Paolucci, and A. Quarteroni, 2d and 3d elastic wave propagation by a pseudo-spectral domain decomposition method, Journal of Seismology, 1(3), 237-251, doi:10.1023/A:1009758820546, 1997.

Fichtner, A., Full Seismic Waveform Modelling and Inversion, Springer Berlin Heidelberg, doi:10.1007/978-3-642-15807-0, 2010.

Flesch, L. M., A. J. Haines, and W. E. Holt, Dynamics of the India-Eurasia collision zone, Journal of Geophysical Research: Solid Earth, 106(B8), 16,435-16,460, doi: 10.1029/2001jb000208, 2001.

Flinn, E., Signal analysis using rectilinearity and direction of particle motion, Proceedings of the IEEE, 53(12), 1874-1876, doi:10.1109/proc.1965.4462, 1965.

Fouch, M. J., and S. Rondenay, Seismic anisotropy beneath stable continental interiors, Physics of the Earth and Planetary Interiors, 158(2), 292320, doi:10.1016/j.pepi. 2006.03.024, continental Anisotropy, 2006. 
Fry, B., F. Davey, D. Eberhart-Phillips, and S. Lebedev, Depth variable crustal anisotropy, patterns of crustal weakness, and destructive earthquakes in Canterbury, New Zealand, Earth and Planetary Science Letters, 392, 50-57, 2014.

Fukao, Y., Evidence from core-reflected shear waves for anisotropy in the Earth's mantle, Nature, 309(5970), 695-698, doi:10.1038/309695a0, 1984.

Gao, Y., and S. Crampin, Observations of stress relaxation before earthquakes, Geophysical Journal International, 157(2), 578-582, doi:10.1111/j.1365-246x.2004. 02207.x, 2004.

Gao, Y., and S. Crampin, Retrospective stress-forecasting of earthquakes, Geophysical Research Abstracts, 17, EGU2015-5984, 2015.

Gerst, A., and M. Savage, Seismic anisotropy beneath Ruapehu Volcano: A possible eruption forecasting tool, Science, 306, 1543-1547, 2004.

Gibbons, S. J., and F. Ringdal, The detection of low magnitude seismic events using array-based waveform correlation, Geophysical Journal International, 165(1), 149166, doi:10.1111/j.1365-246x.2006.02865.x, 2006.

Gillam, B. G., T. A. Little, E. Smith, and V. G. Toy, Extensional shear band development on the outer margin of the Alpine mylonite zone, Tatare Stream, Southern Alps, New Zealand, Journal of Structural Geology, 54, 1-20, doi:10.1016/j.jsg.2013.06.010, 2013.

Gledhill, K. R., Evidence for shallow and pervasive seismic anisotropy in the Wellington Region, New Zealand, Journal of Geophysical Research: Solid Earth, 96(B13), 21,503-21,516, doi:10.1029/91JB02049, 1991.

Godfrey, N., N. Christensen, and D. Okaya, Anisotropy of schists: Contribution of crustal anisotropy to active source seismic experiments and shear wave splitting observations, Journal of Geophysical Research: Solid Earth, 105(B12), 27,991-28,007, 2000. 
Groenenboom, J., and R. Snieder, Attenuation, dispersion, and anisotropy by multiple scattering of transmitted waves through distributions of scatterers, The Journal of the Acoustical Society of America, 98(6), 3482-3492, doi:10.1121/1.413780, 1995.

Hamling, I. J., E. D’Anastasio, L. M. Wallace, S. Ellis, M. Motagh, S. Samsonov, N. Palmer, and S. Hreinsdóttir, Crustal deformation and stress transfer during a propagating earthquake sequence: The 2013 Cook Strait sequence, central New Zealand, Journal of Geophysical Research: Solid Earth, 119(7), 6080-6092, doi: 10.1002/2014jb011084, 2014.

Hamling, I. J., S. Hreinsdóttir, K. Clark, J. Elliott, C. Liang, E. Fielding, N. Litchfield, P. Villamor, L. Wallace, T. J. Wright, E. D'Anastasio, S. Bannister, D. Burbidge, P. Denys, P. Gentle, J. Howarth, C. Mueller, N. Palmer, C. Pearson, W. Power, P. Barnes, D. J. A. Barrell, R. V. Dissen, R. Langridge, T. Little, A. Nicol, J. Pettinga, J. Rowland, and M. Stirling, Complex multifault rupture during the 2016 Mw 7.8 Kaikōura earthquake, New Zealand, Science, 356(6334), 1 - 10, doi: dio:10.1126/science.aam7194, 2017.

Hammond, J. O. S., J.-M. Kendall, D. Angus, and J. Wookey, Interpreting spatial variations in anisotropy: insights into the Main Ethiopian Rift from SKS waveform modelling, Geophysical Journal International, doi:10.1111/j.1365-246x.2010.04587.x, 2010.

Hardebeck, J. L., and A. J. Michael, Damped regional-scale stress inversions: Methodology and examples for southern California and the Coalinga aftershock sequence, Journal of Geophysical Research: Solid Earth, 111 (B11), doi:10.1029/2005jb004144, 2006.

Hardebeck, J. L., and T. Okada, Temporal stress changes caused by earthquakes: A review, Journal of Geophysical Research: Solid Earth, 123(2), 1350-1365, doi: 10.1002/2017jb014617, 2018.

Havskov, J., and L. Ottemöller, SEISAN: the earthquake analysis software, For Windows, Solaris, Linux and MACOSX. Version, 8, 2008. 
Healy, D., R. H. Sibson, Z. Shipton, and R. Butler, Stress, faulting, fracturing and seismicity: the legacy of Ernest Masson Anderson, Geological Society, London, Special Publications, 367(1), 1-6, doi:10.1144/sp367.1, 2012.

Hirschberg, H. P., S. Lamb, and M. K. Savage, Strength of an obliquely convergent plate boundary: lithospheric stress magnitudes and viscosity in New Zealand, Geophysical Journal International, 216(2), 1005-1024, doi:10.1093/gji/ggy477, 2018.

Holden, C., A. Kaiser, R. Van Dissen, and R. Jury, Sources, ground motion and structural response characteristics in Wellington of the 2013 Cook Strait earthquakes, Bull. New Zeal. Soc. Earthq. Eng., 46(4), 188-195, 2013.

Holden, C., Y. Kaneko, E. D'Anastasio, R. Benites, B. Fry, and I. Hamling, The 2016 Kaikōura Earthquake Revealed by Kinematic Source Inversion and Seismic Wavefield Simulations: Slow Rupture Propagation on a Geometrically Complex Crustal Fault Network, Geophysical Research Letters, 44(22), doi:10.1002/2017GL075301, 2017.

Holt, R., M. Savage, J. Townend, E. Syracuse, and C. Thurber, Crustal stress and fault strength in the Canterbury Plains, New Zealand, Earth and Planetary Science Letters, 383, 173-181, doi:10.1016/j.epsl.2013.09.041, 2013.

Holt, W. E., and A. J. Haines, The kinematics of northern South Island, New Zealand, determined from geologic strain rates, Journal of Geophysical Research: Solid Earth, 100 (B9), 17,991-18,010, doi:10.1029/95JB01059, 1995.

Howarth, J. D., S. J. Fitzsimons, R. J. Norris, R. Langridge, and M. J. Vandergoes, A 2000 yr rupture history for the Alpine fault derived from Lake Ellery, South Island, New Zealand, Geological Society of America Bulletin, 128(3-4), 627-643, doi:10.1130/b31300.1, 2015.

Howarth, J. D., U. A. Cochran, R. M. Langridge, K. Clark, S. J. Fitzsimons, K. Berryman, P. Villamor, and D. T. Strong, Past large earthquakes on the Alpine Fault: paleoseismological progress and future directions, New Zealand Journal of Geology and Geophysics, 61(3), 309-328, doi:10.1080/00288306.2018.1464658, 2018. 
Hudson, J. A., Wave speeds and attenuation of elastic waves in material containing cracks, Geophysical Journal International, 64(1), 133-150, doi:10.1111/j.1365-246x. 1981.tb02662.x, 1981.

Igel, H., Computational Seismology, Oxford University Press, doi:10.1093/acprof:oso/ 9780198717409.001.0001, 2016.

Igel, H., P. Mora, and B. Riollet, Anisotropic wave propagation through finite-difference grids, Geophysics, 60(4), 1203-1216, doi:10.1190/1.1443849, 1995.

Iidaka, T., and K. Obara, Shear-wave splitting in a region with newly-activated seismicity after the 2011 Tohoku earthquake, Earth, Planets and Space, 65 (9), 1059-1064, doi:10.5047/eps.2013.02.003, 2013.

Illsley-Kemp, F., M. K. Savage, D. Keir, H. P. Hirschberg, J. M. Bull, T. M. Gernon, J. O. Hammond, J.-M. Kendall, A. Ayele, and B. Goitom, Extension and stress during continental breakup: Seismic anisotropy of the crust in Northern Afar, Earth and Planetary Science Letters, 4777, 41-51, doi:10.1016/j.epsl.2017.08.014, 2017.

Ito, H., J. DeVilbiss, and A. Nur, Compressional and shear waves in saturated rock during water-steam transition, Journal of Geophysical Research, 84(B9), 4731, doi: 10.1029/jb084ib09p04731, 1979.

Jenkins, S., R. Sutherland, and J. Townend, Gravity survey of the central Alpine Fault near the DFDP-2 drill site, Whataroa, South Island, New Zealand, New Zealand Journal of Geology and Geophysics, 63(1), 128-144, doi:10.1080/00288306.2019. 1639777, 2019.

Johnson, J. H., S. Prejean, M. K. Savage, and J. Townend, Anisotropy, repeating earthquakes, and seismicity associated with the 2008 eruption of Okmok volcano, Alaska, Journal of Geophysical Research, 115, doi:10.1029/2009jb006991, 2010.

Johnson, J. H., M. K. Savage, and J. Townend, Distinguishing between stress-induced and structural anisotropy at Mount Ruapehu volcano, New Zealand, Journal of Geophysical Research, 116, doi:10.1029/2011JB008308, 2011. 
Johnson, J. H., D. A. Swanson, D. C. Roman, M. P. Poland, and W. A. Thelen, Crustal Stress and Structure at Kīlauea Volcano Inferred from Seismic Anisotropy, in Hawaiian Volcanoes, pp. 251-268, John Wiley \& Sons, Inc, doi:10.1002/9781118872079. $\operatorname{ch} 12,2015$.

Kaiser, A., N. Balfour, B. Fry, C. Holden, N. Litchfield, M. Gerstenberger, and et al., The 2016 Kaikōura, New Zealand, Earthquake: Preliminary Seismological Report, Seismological Research Letters, 88(3), 727-739, doi:10.1785/0220170018, 2017.

Karalliyadda, S. C., and M. K. Savage, Seismic anisotropy and lithospheric deformation of the plate-boundary zone in South Island, New Zealand: inferences from local Swave splitting, Geophysical Journal International, 193(2), 507-530, doi:10.1093/gji/ ggt022, 2013.

Keith, C. M., and S. Crampin, Seismic body waves in anisotropic media: reflection and refraction at a plane interface, Geophysical Journal International, 49(1), 181-208, 1977.

Kisslinger, C., and E. Engdahl, The interpretation of the Wadati diagram with relaxed assumptions, Bulletin of the Seismological Society of America, 63(5), 1723-1736, 1973.

Klosko, E. R., F. T. Wu, H. J. Anderson, D. Eberhart-Phillips, T. V. McEvilly, E. Audoine, M. K. Savage, and K. R. Gledhill, Upper mantle anisotropy in the New Zealand Region, Geophysical Research Letters, 26(10), 1497-1500, doi:10.1029/1999g1900273, 1999.

Komatitsch, D., and J. Tromp, Introduction to the spectral element method for threedimensional seismic wave propagation, Geophysical Journal International, 139(3), 806-822, doi:10.1046/j.1365-246x.1999.00967.x, 1999.

Komatitsch, D., and J. Tromp, Spectral-element simulations of global seismic wave propagation-I. Validation, Geophysical Journal International, 149(2), 390-412, doi: 10.1046/j.1365-246x.2002.01653.x, 2002. 
Komatitsch, D., C. Barnes, and J. Tromp, Simulation of anisotropic wave propagation based upon a spectral element method, Geophysics, 65(4), 1251-1260, doi:10.1190/ $1.1444816,2000$.

Komatitsch, D., S. Tsuboi, and J. Tromp, The Spectral-Element Method in Seismology, pp. 205-227, American Geophysical Union, doi:10.1029/157GM13, 2013.

Kong, Q., D. T. Trugman, Z. E. Ross, M. J. Bianco, B. J. Meade, and P. Gerstoft, Machine Learning in Seismology: Turning Data into Insights, Seismological Research Letters, 90(1), 3-14, doi:10.1785/0220180259, 2018.

Koulakov, I., E. Kukarina, I. H. Fathi, S. E. Khrepy, and N. Al-Arifi, Anisotropic tomography of Hokkaido reveals, Journal of Geophysical Research: Solid Earth, 120, 3219-3239, 2015.

Lamb, S., R. Arnold, and J. D. P. Moore, Locking on a megathrust as a cause of distributed faulting and fault-jumping earthquakes, Nature Geoscience, 11(11), 871875, doi:10.1038/s41561-018-0230-5, 2018.

Langridge, R., K. R. Berryman, and R. J. Van Dissen, Defining the geometric segmentation and Holocene slip rate of the Wellington Fault, New Zealand: The Pahiatua section, New Zealand Journal of Geology and Geophysics, 48(4), 591-607, doi:10.1080/00288306.2005.9515136, 2005.

Langridge, R., W. Ries, T. Farrier, N. Barth, N. Khajavi, and G. D. Pascale, Developing sub 5-m LiDAR DEMs for forested sections of the Alpine and Hope faults, South Island, New Zealand: Implications for structural interpretations, Journal of Structural Geology, 64, 53-66, doi:10.1016/j.jsg.2013.11.007, 2014.

Langridge, R., W. Ries, N. Litchfield, P. Villamor, R. V. Dissen, D. Barrell, M. Rattenbury, D. Heron, S. Haubrock, D. Townsend, J. Lee, K. Berryman, A. Nicol, S. Cox, and M. Stirling, The New Zealand Active Faults Database, New Zealand Journal of Geology and Geophysics, 59(1), 86-96, doi:10.1080/00288306.2015.1112818, 2016. 
Langridge, R. M., J. D. Howarth, S. C. Cox, J. G. Palmer, and R. Sutherland, Frontal fault location and most recent earthquake timing for the Alpine Fault at Whataroa, Westland, New Zealand, New Zealand Journal of Geology and Geophysics, 61(3), 329-340, doi:10.1080/00288306.2018.1509878, 2018.

Lanza, F., C. J. Chamberlain, K. Jacobs, E. Warren-Smith, H. J. Godfrey, M. Kortink, C. H. Thurber, M. K. Savage, J. Townend, S. Roecker, and D. Eberhart-Phillips, Crustal Fault Connectivity of the Mw 7.8 2016 Kaikōura Earthquake Constrained by Aftershock Relocations, Geophysical Research Letters, 46(12), 6487-6496, doi: 10.1029/2019GL082780, 2019.

Lay, V., S. Buske, A. Lukács, A. R. Gorman, S. Bannister, and D. R. Schmitt, Advanced seismic imaging techniques characterize the Alpine Fault at Whataroa (New Zealand), Journal of Geophysical Research: Solid Earth, 121(12), 8792-8812, doi: 10.1002/2016jb013534, 2016.

Lay, V., S. Buske, S. B. Bodenburg, J. Townend, R. Kellett, M. K. Savage, D. R. Schmitt, A. Constantinou, J. D. Eccles, M. Bertram, K. Hall, D. Lawton, A. R. Gorman, and R. S. Kofman, Seismic P Wave Velocity Model From 3-D Surface and Borehole Seismic Data at the Alpine Fault DFDP-2 Drill Site (Whataroa, New Zealand), Journal of Geophysical Research: Solid Earth, 125(4), e2019JB018,519, doi:10.1029/2019JB018519, 2020.

Leary, P. C., S. Crampin, and T. V. McEvilly, Seismic fracture anisotropy in the earth's crust: An overview, Journal of Geophysical Research, 95(B7), 11,105, doi: 10.1029/jb095ib07p11105, 1990.

Lee, S.-J., D. Komatitsch, B.-S. Huang, and J. Tromp, Effects of Topography on Seismic-Wave Propagation: An Example from Northern TaiwanEffects of Topography on Seismic-Wave Propagation: An Example from Northern Taiwan, Bulletin of the Seismological Society of America, 99(1), 314-325, doi:10.1785/0120080020, 2009a. 
Lee, S.-J., Y.-C. Chan, D. Komatitsch, B.-S. Huang, and J. Tromp, Effects of Realistic Surface Topography on Seismic Ground Motion in the Yangminshan Region of Taiwan Based Upon the Spectral-Element Method and LiDAR DEM Effects of Realistic Surface Topography on Seismic Ground Motion in the Yangminshan Region of Taiwan, Bulletin of the Seismological Society of America, 99(2A), 681-693, doi:10.1785/0120080264, 2009b.

Leitner, B., D. Eberhart-Phillips, H. Anderson, and J. L. Nabelek, A focused look at the Alpine fault, New Zealand: Seismicity, focal mechanisms, and stress observations, Journal of Geophysical Research: Solid Earth, 106(B2), 2193-2220, doi:10.1029/ 2000jb900303, 2001.

Levin, V., and J. Park, P-SH conversions in a flat-layered medium with anisotropy of arbitrary orientation, Geophysical Journal International, pp. 253-266, 1997.

Li, Y.-G., Rock Anisotropy, Fracture and Earthquake Assessment, Walter de Gruyter GmbH \& Co KG, 2016.

Li, Y.-G., and J. E. Vidale, Healing of the shallow fault zone from 1994-1998 After the 1992 M7.5 Landers, California, Earthquake, Geophysical Research Letters, 28(15), 2999-3002, doi:10.1029/2001gl012922, 2001.

Li, Y.-G., J. E. Vidale, K. Aki, F. Xu, and T. Burdette, Evidence of Shallow Fault Zone Strengthening After the 1992 M7.5 Landers, California, Earthquake, Science, 279 (5348), 217-219, doi:10.1126/science.279.5348.217, 1998.

Lienert, B., and J. Havskov, A computer program for locating earthquakes both locally and globally, Seismological Research Letters, 66 (5), 26-36, 1995.

Lisitsa, V., and D. Vishnevskiy, Lebedev scheme for the numerical simulation of wave propagation in 3D anisotropic elasticity, Geophysical Prospecting, 58(4), 619-635, doi:10.1111/j.1365-2478.2009.00862.x, 2010.

Litchfield, N., R. V. Dissen, R. Sutherland, P. Barnes, S. Cox, R. Norris, R. Beavan, R. Langridge, P. Villamor, K. Berryman, M. Stirling, A. Nicol, S. Nodder, 
G. Lamarche, D. Barrell, J. Pettinga, T. Little, N. Pondard, J. Mountjoy, and

K. Clark, A model of active faulting in New Zealand, New Zealand Journal of Geology and Geophysics, 57(1), 32-56, doi:10.1080/00288306.2013.854256, 2013.

Litchfield, N. J., A. Benson, A. Bischoff, A. Hatem, A. Wandres, and et. al., Multiple Fault Ground Surface Ruptures In The 14 November 2016 Kaikōura Earthquake, New Zealand, Seismological Research Letters, 88(2B), 624-626, doi: 10.1785/0220170035, 2017.

Little, T., R. Holcombe, and B. Ilg, Kinematics of oblique collision and ramping inferred from microstructures and strain in middle crustal rocks, central Southern Alps, New Zealand, Journal of Structural Geology, 24(1), 219-239, doi:10.1016/s0191-8141(01) 00060-8, 2002.

Little, T. A., S. Cox, J. K. Vry, and G. Batt, Variations in exhumation level and uplift rate along the obliqu-slip Alpine fault, central Southern Alps, New Zealand, Geological Society of America Bulletin, 117(5), 707, doi:10.1130/b25500.1, 2005.

Liu, E., and S. Crampin, Effects of the internal shear wave window: Comparison with anisotropy induced splitting, Journal of Geophysical Research: Solid Earth, 95(B7), 11,275-11,281, doi:10.1029/JB095iB07p11275, 1990.

Liu, Y., Systematic Analysis of Shear-Wave Splitting in the Aftershock Zone of the 1999 Chi-Chi, Taiwan, Earthquake: Shallow Crustal Anisotropy and Lack of Precursory Variations, Bulletin of the Seismological Society of America, 94(6), 2330-2347, doi: 10.1785/0120030139, 2004.

Liu, Y., S. Crampin, and I. Main, Shear-wave anisotropy: spatial and temporal variations in time delays at Parkfield, Central California, Geophysical Journal International, 130(3), 771-785, 1997.

Lucente, F. P., P. D. Gori, L. Margheriti, D. Piccinini, M. D. Bona, C. Chiarabba, and N. P. Agostinetti, Temporal variation of seismic velocity and anisotropy before the 
2009 MW6.3 L'Aquila earthquake, Italy, Geology, 38(11), 1015-1018, doi:10.1130/ g31463.1, 2010.

Massiot, C., B. Célérier, M.-L. Doan, T. A. Little, J. Townend, D. D. McNamara, J. Williams, D. R. Schmitt, V. G. Toy, R. Sutherland, L. Janku-Capova, P. Upton, and P. A. Pezard, The Alpine Fault Hangingwall Viewed From Within: Structural Analysis of Ultrasonic Image Logs in the DFDP-2B Borehole, New Zealand, Geochemistry, Geophysics, Geosystems, 19(8), 2492-2515, doi:10.1029/2017gc007368, 2018.

Matcham, I., M. K. Savage, and K. R. Gledhill, Distribution of seismic anisotropy in the subduction zone beneath the Wellington region, New Zealand, Geophysical Journal International, 140(1), 1-10, doi:10.1046/j.1365-246x.2000.00928.x, 2000.

Maupin, V., and J. Park, Theory and observations - wave propagation in anisotropic media, Treatise on geophysics, 1, 289-321, 2007.

McLaskey, G. C., A. M. Thomas, S. D. Glaser, and R. M. Nadeau, Fault healing promotes high-frequency earthquakes in laboratory experiments and on natural faults, Nature, 491 (7422), 101-104, doi:10.1038/nature11512, 2012.

McNab, A., Velocity structure of the Whataroa Valley using Ambient Noise Tomography, Master's thesis, Victoria University of Wellington, 2017.

Menke, W., and V. Levin, The cross-convolution method for interpreting SKS splitting observations, with application to one and two-layer anisotropic earth models, Geophysical Journal International, 154(2), 379-392, doi:10.1046/j.1365-246x.2003. 01937.x, 2003.

Michael, A. J., Determination of stress from slip data: Faults and folds, Journal of Geophysical Research: Solid Earth, 89(B13), 11,517-11,526, doi:10.1029/ JB089iB13p11517, 1984.

Michailos, K., E. G. C. Smith, C. J. Chamberlain, M. K. Savage, and J. Townend, Variations in seismogenic thickness along the central Alpine Fault, New Zealand, 
revealed by a decade's relocated microseismicity, Geochemistry, Geophysics, Geosystems, doi:10.1029/2018gc007743, 2018.

Michailos, K., E. Warren-Smith, M. K. Savage, and J. Townend, Detailed spatiotemporal analysis of the tectonic stress regime near the central Alpine Fault, New Zealand, Tectonophysics, 775, 228,205, doi:10.1016/j.tecto.2019.228205, 2020.

Miller, V., and M. Savage, Changes in seismic anisotropy after volcanic eruptions: evidence from Mount Ruapehu, Science, 293(5538), 2231-2233, 2001.

Mishra, O., and D. Zhao, Crack density, saturation rate and porosity at the 2001 Bhuj, India, earthquake hypocenter: a fluid-driven earthquake?, Earth and Planetary Science Letters, 212(3-4), 393-405, doi:10.1016/s0012-821x(03)00285-1, 2003.

Mitchell, J., K. Mackay, H. Neil, E. Mackay, A. Pallentin, and P. Notman, New Zealand Region Bathymetry, 1:4,000000, 2012.

Moczo, P., J. O. Robertsson, and L. Eisner, The Finite-Difference Time-Domain Method for Modeling of Seismic Wave Propagation, in Advances in Wave Propagation in Heterogenous Earth, pp. 421-516, Elsevier, doi:10.1016/s0065-2687(06)48008$0,2007$.

Monteiller, V., and S. Chevrot, How to make robust splitting measurements for singlestation analysis and three-dimensional imaging of seismic anisotropy, Geophysical Journal International, doi:10.1111/j.1365-246x.2010.04608.x, 2010.

Mortimer, N., Metamorphic zones, terranes, and Cenozoic faults in the Marlborough Schist, New Zealand, New Zealand Journal of Geology and Geophysics, 36(3), 357368, doi:10.1080/00288306.1993.9514581, 1993.

Mortimer, N., New Zealand's geological foundations, Gondwana Research, 7(1), 261$272,2004$.

Nakata, N., and R. Snieder, Time-lapse change in anisotropy in Japan's near surface after the 2011 Tohoku-Oki earthquake, Geophysical Research Letters, 39(11), doi: 10.1029/2012gl051979, 2012. 
NCEI Geomagnetic Modeling Team, and British Geological Survey, World magnetic model 2020, doi:10.25921/11V3-DA71, 2019.

Neuberg, J., and T. Pointer, Effects of volcano topography on seismic broad-band waveforms, Geophysical Journal International, 143(1), 239-248, doi:10.1046/j.1365246x.2000.00251.x, 2000.

Norris, R. J., and A. F. Cooper, Late Quaternary slip rates and slip partitioning on the Alpine Fault, New Zealand, Journal of Structural Geology, 23(2-3), 507-520, doi:10.1016/s0191-8141(00)00122-x, 2001.

Norris, R. J., and V. G. Toy, Continental transforms: A view from the Alpine Fault, Journal of Structural Geology, 64, 3-31, doi:10.1016/j.jsg.2014.03.003, 2014.

Norris, R. J., A. Cooper, V. Toy, S. Read, and L. Easterbrook, Three-dimensional structure of the Alpine Fault zone in the region around the Waitangi-taona and Whataroa Rivers, 2002 Geosciences New Zealnd, 2012.

Nur, A., Effects of stress on velocity anisotropy in rocks with cracks, Journal of Geophysical Research, 76(8), 2022-2034, doi:10.1029/jb076i008p02022, 1971.

Nuttli, O., The effect of the Earth's surface on the S wave particle motion, Bulletin of the Seismological Society of America, 51(2), 237, 1961.

O’Connell, R. J., and B. Budiansky, Seismic velocities in dry and saturated cracked solids, Journal of Geophysical Research, 79(35), 5412-5426, doi:10.1029/ JB079i035p05412, 1974.

Okada, T., Y. Iio, S. Matsumoto, S. Bannister, S. Ohmi, S. Horiuchi, T. Sato, T. Miura, J. Pettinga, F. Ghisetti, and R. H. Sibson, Comparative tomography of reverse-slip and strike-slip seismotectonic provinces in the northern South Island, New Zealand, Tectonophysics, 765, 172-186, doi:10.1016/j.tecto.2019.03.016, 2019.

Okaya, D., N. Christensen, D. Stanley, T. Stern, and S. (term), Crustal anisotropy in the vicinity of the Alpine Fault Zone, South Island, New Zealand, New Zealand Jour- 
nal of Geology and Geophysics, 38(4), 579-583, doi:10.1080/00288306.1995.9514686, 1995.

Okaya, D., S. Henrys, and T. Stern, Double-sided onshore-offshore seismic imaging of a plate boundary: 'super-gathers' across South Island, New Zealand, Tectonophysics, 355(1-4), 247-263, doi:10.1016/s0040-1951(02)00145-2, 2002.

Okaya, D., N. I. Christensen, Z. E. Ross, and F. T. Wu, Terrane-controlled crustal shear wave splitting in Taiwan, Geophysical Research Letters, 43(2), 556-563, doi: 10.1002/2015gl066446, 2016.

O'Keefe, B. C., Microseismicity of the Central Alpine fault Region, New Zealand, Master's thesis, Victoria University of Wellington, 2008.

Park, J., Surface waves in layered anisotropic structures, Geophysical Journal International, 126(1), 173-183, doi:10.1111/j.1365-246x.1996.tb05276.x, 1996.

Pavlov, V. A., 3D Partitioning in SPECFEM3D Internal Mesher, 2012.

Peng, Z., and Y. Ben-Zion, Systematic analysis of crustal anisotropy along the Karadere-Düzce branch of the North Anatolian fault, Geophysical Journal International, $159(1), 253-274,2004$.

Peng, Z., and Y. Ben-Zion, Spatio-temporal variations of crustal anisotropy from similar events in aftershocks of the 1999 M7.4 Izmit and M7.1 Duzce, Turkey, earthquake sequences, Geophys. J. Int., 160, 1027-1043, 2005.

Peter, D., D. Komatitsch, Y. Luo, R. Martin, N. Le Goff, E. Casarotti, P. Le Loher, F. Magnoni, Q. Liu, C. Blitz, T. Nissen-Meyer, P. Basini, and J. Tromp, Forward and adjoint simulations of seismic wave propagation on fully unstructured hexahedral meshes, Geophysical Journal International, 186(2), 721-739, doi: 10.1111/j.1365-246X.2011.05044.x, 2011.

Petersen, T., K. Gledhill, M. Chadwick, N. H. Gale, and J. Ristau, The New Zealand National Seismograph Network, Seismological Research Letters, 82(1), 9-20, doi: 10.1785/gssrl.82.1.9, 2011. 
Pettinga, J., M. Yetton, R. Van Dissen, and G. Downes, Earthquake Source Identification and Characterisation for the Canterbury Region, South Island, New Zealand, Bulletin of the New Zealand Society for Earthquake Engineering, 34(4), 282-317, 2001.

Piccinini, D., L. Margheriti, L. Chiaraluce, and M. Cocco, Space and time variations of crustal anisotropy during the 1997 Umbria-Marche, central Italy, seismic sequence, Geophysical Journal International, 167(3), 1482-1490, doi:10.1111/j.1365246x.2006.03112.x, 2006.

Priolo, E., J. M. Carcione, and G. Seriani, Numerical simulation of interface waves by high-order spectral modeling techniques, The Journal of the Acoustical Society of America, 95(2), 681-693, doi:10.1121/1.408428, 1994.

Pulford, A., M. Savage, and T. Stern, Absent anisotropy: The paradox of the Southern Alps orogen, Geophysical Research Letters, 30(20), doi:10.1029/2003g1017758, 2003.

Qinya, L., P. Jascha, K. Dimitri, and T. Jeroen, Spectral-element moment tensor inversions for earthquakes in Southern California, Bulletin of the Seismological Society of America, 94(5), 1748-1761, 2004.

Reyners, M., and H. Cowan, The transition from subduction to continental collision: crustal structure in the North Canterbury region, New Zealand, Geophysical Journal International, 115(3), 1124-1136, doi:10.1111/j.1365-246X.1993.tb01514.x, 1993.

Ristau, J., Implementation of Routine Regional Moment Tensor Analysis in New Zealand, Seismological Research Letters, 79(3), 400-415, doi:10.1785/gssrl.79.3.400, 2008.

Ristau, J., Update of Regional Moment Tensor Analysis for Earthquakes in New Zealand and Adjacent Offshore Regions, Bulletin of the Seismological Society of America, 103(4), 2520-2533, doi:10.1785/0120120339, 2013.

Robertsson, J. O. A., J. O. Blanch, K. Nihei, and J. Tromp, Numerical Modeling 
of Seismic Wave Propagation, Society of Exploration Geophysicists, doi:10.1190/1. $9781560803089,2012$.

Rodgers, A. J., N. A. Petersson, and B. Sjogreen, Simulation of topographic effects on seismic waves from shallow explosions near the North Korean nuclear test site with emphasis on shear wave generation, Journal of Geophysical Research, 115(B11), doi:10.1029/2010jb007707, 2010.

Roman, D. C., M. K. Savage, R. Arnold, J. L. Latchman, and S. De Angelis, Analysis and forward modeling of seismic anisotropy during the ongoing eruption of the Soufrière Hills Volcano, Montserrat, 1996-2007, Journal of Geophysical Research: Solid Earth, 116(B3), doi:10.1029/2010jb007667, 2011.

Ross, Z. E., M.-A. Meier, E. Hauksson, and T. H. Heaton, Generalized seismic phase detection with deep learning, Bulletin of the Seismological Society of America, 108(5A), 2894-2901, doi:10.1785/0120180080, 2018.

Rümpker, G., and P. G. Silver, Apparent shear-wave splitting parameters in the presence of vertically varying anisotropy, Geophysical Journal International, 135(3), 790800, doi:10.1046/j.1365-246x.1998.00660.x, 1998.

Rümpker, G., and P. G. Silver, Apparent shear-wave splitting parameters in the presence of vertically varying anisotropy, Geophysical Journal International, 135(3), 790-800, 1998.

Saenger, E. H., and T. Bohlen, Finite-difference modeling of viscoelastic and anisotropic wave propagation using the rotated staggered grid, in Numerical Modeling of Seismic Wave Propagation: Gridded Two-way Wave-equation Methods, Society of Exploration Geophysicists, doi:10.1190/1.9781560803089, 2012.

Saiga, A., Y. Hiramatsu, T. Ooida, and K. Yamaoka, Spatial variation in the crustal anisotropy and its temporal variation associated with a moderate-sized earthquake in the Tokai region, central Japan, Geophysical Journal International, 154(3), 695-705, doi:10.1046/j.1365-246x.2003.01998.x, 2003. 
Saltzer, R. L., J. B. Gaherty, and T. H. Jordan, How are vertical shear wave splitting measurements affected by variations in the orientation of azimuthal anisotropy with depth?, Geophysical Journal International, 141(2), 374-390, doi:10.1046/j.1365246x.2000.00088.x, 2000.

Sato, H., M. C. Fehler, and T. Maeda, Seismic wave propagation and scattering in the heterogeneous earth, vol. 484, Springer, doi:10.1007/978-3-540-89623-4, 2012.

Savage, M., Seismic anisotropy and mantle deformation: what have we learned from shear wave splitting?, Rev. Geophys., 37, 65-106, 1999.

Savage, M., X. Shih, R. Meyer, and R. Aster, Shear-wave anisotropy of active tectonic regions via automated S-wave polarization analysis, Tectonophysics, 165(1-4), 279292, doi:10.1016/0040-1951(89)90053-x, 1989.

Savage, M., A. Wessell, N. Teanby, and A. Hurst, Automatic measurement of shear wave splitting and applications to time varying anisotropy at Mount Ruapehu volcano, New Zealand, Journal of Geophysical Research, 115, 115, doi: 10.1029/2010jb007722, 2010a.

Savage, M., T. Ohminato, Y. Aoki, H. Tsuji, and S. M. Greve, Stress magnitude and its temporal variation at Mt. Asama Volcano, Japan, from seismic anisotropy and GPS, Earth and Planetary Science Letters, 290(3), 403 - 414, doi:10.1016/j.epsl. 2009.12.037, 2010b.

Savage, M., Y. Aoki, K. Unglert, T. Ohkura, K. Umakoshi, H. Shimizu, M. Iguchi, T. Tameguri, T. Ohminato, and J. Mori, Stress, strain rate and anisotropy in Kyushu, Japan, Earth and Planetary Science Letters, 439, 129-142, doi:10.1016/j.epsl.2016. 01.005, 2016.

Savage, M. K., K. M. Fischer, and C. E. Hall, Strain modelling, seismic anisotropy and coupling at strike-slip boundaries: applications in New Zealand and the San Andreas fault, Geological Society, London, Special Publications, 227(1), 9-39, doi: 10.1144/gsl.sp.2004.227.01.02, 2004. 
Savage, M. K., A. Tommasi, S. Ellis, and J. Chery, Modeling strain and anisotropy along the Alpine Fault, South Island, New Zealand, in A Continental Plate Boundary: Tectonics at South Island, New Zealand, pp. 289-305, American Geophysical Union, doi:10.1029/175gm15, 2007.

Savage, M. K., V. Ferrazzini, A. Peltier, E. Rivemale, J. Mayor, A. Schmid, F. Brenguier, F. Massin, J.-L. Got, J. Battaglia, A. DiMuro, T. Staudacher, D. Rivet, B. Taisne, and A. Shelley, Seismic anisotropy and its precursory change before eruptions at Piton de la Fournaise volcano, La Réunion, Journal of Geophysical Research Solid Earth, 120(5), 3430-3458, doi:10.1002/2014jb011665, 2015.

Schoenberg, M., and F. Muir, A calculus for finely layered anisotropic media, Geophysics, 54 (5), 581-589, doi:10.1190/1.1442685, 1989.

Shearer, P., Deep earth structure: Seismic scattering in the deep earth, in Treatise on Geophysics, pp. 759-787, Elsevier, doi:10.1016/b978-0-444-53802-4.00018-x, 2015.

Shearer, P. M., and J. A. Orcutt, Compressional and shear wave anisotropy in the oceanic lithosphere - the Ngendei seismic refraction experiment, Geophysical Journal International, 87(3), 967-1003, doi:10.1111/j.1365-246x.1986.tb01979.x, 1986.

Shih, X. R., and R. P. Meyer, Observation of shear wave splitting from natural events: South Moat of Long Valley Caldera, California, June 29 to August 12, 1982, Journal of Geophysical Research: Solid Earth, 95(B7), 11,179-11,195, doi: 10.1029/JB095iB07p11179, 1990.

Shih, X. R., R. P. Meyer, and J. F. Schneider, An automated, analytical method to determine shear-wave splitting, Tectonophysics, 165(1), 271 - 278, doi:https://doi. org/10.1016/0040-1951(89)90052-8, cited By :47, 1989.

Silver, P. G., and W. W. Chan, Shear wave splitting and subcontinental mantle deformation, Journal of Geophysical Research: Solid Earth, 96(B10), 16,429-16,454, 1991. 
Silver, P. G., and M. K. Savage, The interpretation of shear-wave splitting parameters in the presence of two anisotropic layers, Geophys. J. Int., 119(3), 949-963, 1994.

Simpson, J., L. Adam, K. Wijk, and J. Charoensawan, Constraining Microfractures in Foliated Alpine Fault Rocks With Laser Ultrasonics, Geophysical Research Letters, $47(8)$, doi:10.1029/2020gl087378, 2020.

Stein, S., and M. Wysession, An Introduction to Sesimology, Earthquakes and Earth Structure, Blackwell Publishing, MA, Blackwell Publishing, MA, USA, 2003.

Sutherland, R., Cenozoic bending of New Zealand basement terranes and Alpine Fault displacement: a brief review, New Zealand Journal of Geology and Geophysics, 42(2), 295-301, 1999.

Sutherland, R., D. Eberhart-Phillips, R. Harris, T. Stern, J. Beavan, S. Ellis, S. Henrys, S. Cox, R. Norris, K. Berryman, and et al., Do great earthquakes occur on the Alpine fault in central South Island, New Zealand?, A continental plate boundary: tectonics at South Island, New Zealand, pp. 235-251, 2007.

Sutherland, R., V. G. Toy, J. Townend, S. C. Cox, J. D. Eccles, D. R. Faulkner, D. J. Prior, R. J. Norris, E. Mariani, C. Boulton, B. M. Carpenter, C. D. Menzies, T. A. Little, M. Hasting, G. P. D. Pascale, R. M. Langridge, H. R. Scott, Z. R. Lindroos, B. Fleming, and A. J. Kopf, Drilling reveals fluid control on architecture and rupture of the Alpine fault, New Zealand, Geology, 40(12), 1143-1146, doi:10.1130/g33614.1, 2012.

Sutherland, R., J. Townend, V. Toy, P. Upton, J. Coussens, M. Allen, L.-M. Baratin, and et al., Extreme hydrothermal conditions at an active plate-bounding fault, $\mathrm{Na}$ ture, 546(7656), 137-140, doi:10.1038/nature22355, 2017.

Tadokoro, K., and M. Ando, Evidence for rapid fault healing derived from temporal changes in S wave splitting, Geophysical Research Letters, 29(4), 6-1-6-4, doi:10. 1029/2001gl013644, 2002. 
Tadokoro, K., M. Ando, and Y. Umeda, S-wave splitting in the aftershock region of the 1995 Hyogo-ken Nanbu earthquake, Journal of Geophysical Research: Solid Earth, 104(B1), 981-991, doi:10.1029/1998jb900024, 1999.

Tadokoro, K., M. Ando, Ş. Bariş, K. Nishigami, M. Nakamura, S. B. Ücer, A. Ito, Y. Honkura, and A. M. Işikara, Monitoring of fault healing after the 1999 Kocaeli, Turkey, earthquake, Journal of Seismology, 6(3), 411-417, doi:10.1023/a: $1020091610510,2002$.

Takanami, T., and G. Kitagawa, A new efficient procedure for the estimation of onset times of seismic waves., Journal of Physics of the Earth, 36(6), 267-290, doi:10. 4294/jpe1952.36.267, 1988.

Teanby, N., J.-M. Kendall, R. Jones, and O. Barkved, Stress-induced temporal variations in seismic anisotropy observed in microseismic data, Geophysical Journal International, 156(3), 459-466, doi:10.1111/j.1365-246x.2004.02212.x, 2004a.

Teanby, N., J.-M. Kendall, and M. Van der Baan, Automation of shear-wave splitting measurements using cluster analysis, Bulletin of the Seismological Society of America, 94(2), 453-463, $2004 b$.

Townend, J., and M. D. Zoback, How faulting keeps the crust strong, Geology, 28(5), 399, 2000.

Townend, J., and M. D. Zoback, Implications of earthquake focal mechanisms for the frictional strength of the San Andreas fault system, Geological Society, London, Special Publications, 186(1), 13-21, doi:10.1144/gsl.sp.2001.186.01.02, 2001.

Townend, J., R. Sutherland, and V. Toy, Deep Fault Drilling Project; Alpine Fault, New Zealand, Scientific Drilling, 8, 75-82, doi:10.5194/sd-8-75-2009, 2009.

Townend, J., S. Sherburn, R. Arnold, C. Boese, and L. Woods, Three-dimensional variations in present-day tectonic stress along the Australia-Pacific plate boundary in New Zealand, Earth and Planetary Science Letters, 353-354, 47-59, doi:10.1016/ j.epsl.2012.08.003, 2012. 
Townend, J., R. Sutherland, V. G. Toy, J. D. Eccles, C. Boulton, S. C. Cox, and D. McNamara, Late-interseismic state of a continental plate-bounding fault: Petrophysical results from DFDP-1 wireline logging and core analysis, Alpine Fault, New Zealand, Geochemistry, Geophysics, Geosystems, 14(9), 3801-3820, doi:10.1002/ggge.20236, 2013.

Townend, J., R. Sutherland, V. G. Toy, M.-L. Doan, B. Célérier, C. Massiot, and et al., Petrophysical, Geochemical, and Hydrological Evidence for Extensive Fracture-Mediated Fluid and Heat Transport in the Alpine Fault's Hanging-Wall Damage Zone, Geochemistry, Geophysics, Geosystems, 18(12), 4709-4732, doi: 10.1002/2017gc007202, 2017.

Townend, J., J. Eccles, R. Kellett, and DFDP Seismic Project Team, Whataroa 2016 Seismic Experiment Acquisition Report, doi:10.21420/G2TK9T, 2018.

Toy, V. G., R. Sutherland, J. Townend, M. J. Allen, L. Becroft, A. Bolesand, and et al., Bedrock geology of DFDP-2B, central Alpine Fault, New Zealand, New Zealand Journal of Geology and Geophysics, 60(4), 497-518, doi:10.1080/00288306.2017.1375533, 2017.

Tromp, J., D. Komatitsch, and D. Liu, Spectral-element and adjoint methods in seismology, Communications in Computational Physics, 3, 1-32, 2008.

Turcotte, D. L., The State of Stress at Passive Continental Margins, pp. 141-146, American Geophysical Union (AGU), doi:10.1029/GD006p0141, 1982.

Unglert, K., M. K. Savage, N. Fournier, T. Ohkura, and Y. Abe, Shear wave splitting, Vp/Vs, and GPS during a time of enhanced activity at Aso caldera, Kyushu, Journal of Geophysical Research: Solid Earth, 116(B11), doi:10.1029/2011jb008520, 2011.

Upton, P., B. R. Song, and P. O. Koons, Topographic control on shallow fault structure and strain partitioning near Whataroa, New Zealand demonstrates weak Alpine Fault, New Zealand Journal of Geology and Geophysics, 61(1), 1-8, doi:10.1080/ 00288306.2017.1397706, 2017. 
Upton, P., U. Cochran, C. Orchiston, J. Howarth, J. Pettinga, and J. Townend, Tercentenary of the $1717 \mathrm{AD}$ alpine fault earthquake: advances in science and understanding hazards, New Zealand Journal of Geology and Geophysics, 61(3), 247-250, doi:10.1080/00288306.2018.1512389, 2018.

Van Dissen, R., and R. S. Yeats, Hope fault, Jordan thrust, and uplift of the Seaward Kaikōura Range, New Zealand, Geology, 19(4), 393, doi:10.1130/0091-7613(1991) 019〈0393:HFJTAU $\rangle 2.3 . C O ; 2,1991$.

Vecsey, L., J. Plomerová, and V. Babuška, Shear-wave splitting measurements — problems and solutions, Tectonophysics, 462(1-4), 178-196, doi:10.1016/j.tecto.2008.01. 021, 2008.

Verdon, J., J.-M. Kendall, and A. Wüstefeld, Imaging fractures and sedimentary fabrics using shear wave splitting measurements made on passive seismic data, Geophysical Journal International, 179(2), 1245-1254, 2009.

Volti, T., and S. Crampin, A four-year study of shear-wave splitting in Iceland: 1. Background and preliminary analysis, Geological Society, London, Special Publications, 212(1), 117-133, doi:10.1144/gsl.sp.2003.212.01.08, 2003a.

Volti, T., and S. Crampin, A four-year study of shear-wave splitting in Iceland: 2. Temporal changes before earthquakes and volcanic eruptions, Geological Society, London, Special Publications, 212(1), 135-149, doi:10.1144/gsl.sp.2003.212.01.09, 2003b.

Wadati, K., and S. Oki, On the Travel Time of Earthquake Waves. (Part II), Journal of the Meteorological Society of Japan. Ser. II, 11(1), 14-28, doi:10.2151/jmsj1923. $11.1 \_14,1933$.

Walker, A. M., and J. Wookey, MSAT-A new toolkit for the analysis of elastic and seismic anisotropy, Computers $\&$ Geosciences, 49, 81-90, 2012.

Wallace, L. M., J. Beavan, R. McCaffrey, K. Berryman, and P. Denys, Balancing the plate motion budget in the South Island, New Zealand using GPS, geological 
and seismological data, Geophysical Journal International, 168(1), 332-352, doi: 10.1111/j.1365-246X.2006.03183.x, 2007.

Wallace, L. M., Y. Kaneko, S. Hreinsdóttir, I. Hamling, Z. Peng, N. Bartlow, E. D'Anastasio, and B. Fry, Large-scale dynamic triggering of shallow slow slip enhanced by overlying sedimentary wedge, Nature Geoscience, 10(10), 765-770, doi: 10.1038/ngeo3021, 2017.

Walsh, E., Measuring shear wave splitting using the Silver and Chan method, Master's thesis, Victoria University of Wellington, 2012.

Walsh, E., R. Arnold, and M. K. Savage, Silver and Chan revisited, Journal of Geophysical Research: Solid Earth, 118(10), 5500-5515, doi:10.1002/jgrb.50386, 2013.

Wang, N., J.-P. Montagner, A. Fichtner, and Y. Capdeville, Intrinsic versus extrinsic seismic anisotropy: The radial anisotropy in reference Earth models, Geophysical Research Letters, 40(16), 4284-4288, doi:10.1002/grl.50873, 2013.

Wannamaker, P. E., T. G. Caldwell, G. R. Jiracek, V. Maris, G. J. Hill, Y. Ogawa, H. M. Bibby, S. L. Bennie, and W. Heise, Fluid and deformation regime of an advancing subduction system at Marlborough, New Zealand, Nature, 460(7256), 733-736, doi:10.1038/nature08204, 2009.

Warren-Smith, E., C. J. Chamberlain, S. Lamb, and J. Townend, High-Precision Analysis of an Aftershock Sequence Using Matched-Filter Detection: The 4 May 2015 ML6 Wanaka Earthquake, Southern Alps, New Zealand, Seismological Research Letters, 88(4), 1065-1077, doi:10.1785/0220170016, 2017.

Wech, A. G., C. M. Boese, T. A. Stern, and J. Townend, Tectonic tremor and deep slow slip on the Alpine Fault, Geophysical Research Letters, 39(10), doi:10.1029/ 2012gl051751, 2012.

Wessel, A., Automatic shear wave splitting measurements at Mt. Ruapehu volcano, New Zealand, Master's thesis, Victoria University of Wellington, 2010. 
Wessel, P., W. H. F. Smith, R. Scharroo, J. Luis, and F. Wobbe, Generic Mapping Tools: Improved Version Released, Eos, Transactions American Geophysical Union, 94(45), 409-410, doi:10.1002/2013EO450001, 2013.

Williams, C. A., D. Eberhart-Phillips, S. Bannister, D. H. N. Barker, S. Henrys, M. Reyners, and R. Sutherland, Revised Interface Geometry for the Hikurangi Subduction Zone, New Zealand, Seismological Research Letters, 84(6), 1066-1073, doi: 10.1785/0220130035, 2013.

Williams, J. N., V. G. Toy, S. A. Smith, and C. Boulton, Fracturing, fluid-rock interaction and mineralisation during the seismic cycle along the Alpine Fault, Journal of Structural Geology, 103, 151-166, doi:10.1016/j.jsg.2017.09.011, 2017.

Wilson, C. K., C. H. Jones, P. Molnar, A. F. Sheehan, and O. S. Boyd, Distributed deformation in the lower crust and upper mantle beneath a continental strike-slip fault zone: Marlborough fault system, South Island, New Zealand, Geology, 32(10), 837, doi:10.1130/g20657.1, 2004.

Wookey, J., and G. Helffrich, Inner-core shear-wave anisotropy and texture from an observation of PKJKP waves, Nature, 454(7206), 873-876, 2008.

Yang, Z., A. Sheehan, and P. Shearer, Stress-induced upper crustal anisotropy in southern California, Journal of Geophysical Research: Solid Earth, 116(B2), 1-11, doi: 10.1029/2010JB007655, 2011.

Zal, H. J., K. Jacobs, M. K. Savage, J. Yarce, S. Mroczek, K. Graham, E. K. Todd, J. Nakai, Y. Iwasaki, A. Sheehan, K. Mochizuki, L. Wallace, S. Schwartz, S. Webb, and S. Henrys, Temporal and spatial variations in seismic anisotropy and Vp/Vs ratios in a region of slow slip, Earth and Planetary Science Letters, 532, 115,970, doi:10.1016/j.epsl.2019.115970, 2020.

Zhang, H., Y. Liu, C. Thurber, and S. Roecker, Three-dimensional shear-wave splitting tomography in the Parkfield, California, region, Geophysical Research Letters, 34 (24), doi:10.1029/2007GL031951, 2007. 
Zheng, X.-F., C.-H. Chen, and C.-H. Zhang, Study on Temporal Variations of ShearWave Splitting in the Chiayi Area, Aftershock Zone of 1999 Chichi Earthquake, Taiwan, Chinese Journal of Geophysics, 51(1), 115-124, doi:10.1002/cjg2.1200, 2008.

Zietlow, D. W., A. F. Sheehan, P. H. Molnar, M. K. Savage, G. Hirth, J. A. Collins, and B. H. Hager, Upper mantle seismic anisotropy at a strike-slip boundary: South Island, New Zealand, Journal of Geophysical Research: Solid Earth, 119(2), 10201040, doi:10.1002/2013jb010676, 2014.

Zinke, J. C., Structure-Related and Stress-Induced Shear-Wave Velocity Anisotropy: Observations from Microearthquakes near the Calaveras Fault in Central California, Bulletin of the Seismological Society of America, 90(5), 1305-1312, doi:10.1785/ 0119990099, 2000.

Zoback, M. D., and M. L. Zoback, Stress in the Earth's lithosphere, in Encyclopedia of physical science and technology, vol. 16, 3 ed., pp. 1221-1232, Kluwer Academic Publishers, 2002. 